\title{
THE ROLE OF NON-EQUILIBRIUM PROCESSES IN GALAXY CLUSTERS
}

\author{
Ka-Wah Wong \\ Hong Kong, China
}

B.S. in Physics, The Chinese University of Hong Kong, 2001

M.Phil. in Physics, The Chinese University of Hong Kong, 2003

M.S. in Astronomy, University of Virginia, 2005

A Dissertation Presented to the Graduate

Faculty of the University of Virginia

in Candidacy for the Degree of

Doctor of Philosophy

\section{Department of Astronomy \\ University of Virginia}

December, 2010

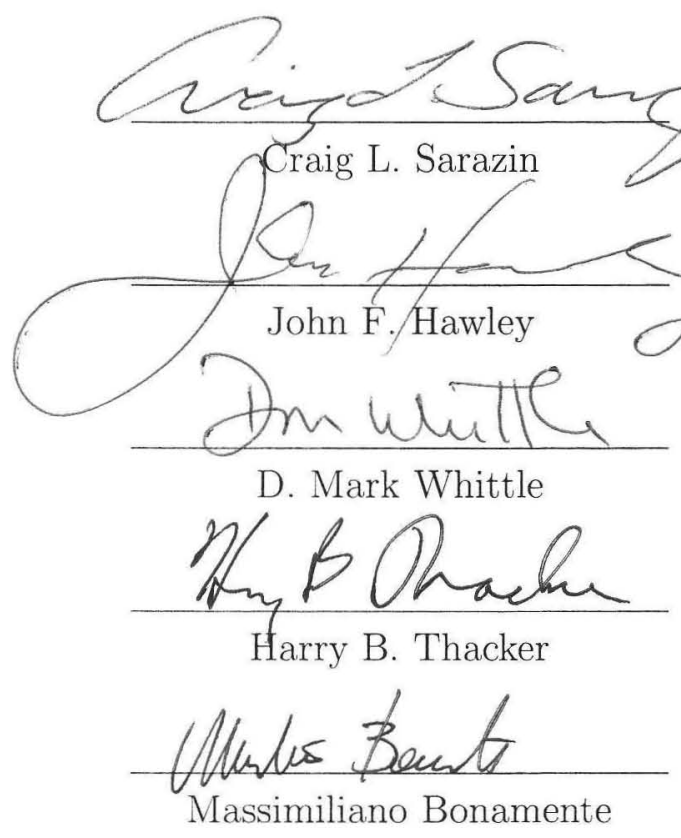


(C) Copyright by Ka-Wah Wong

All rights reserved

December 2010 


\begin{abstract}
This thesis studies non-equilibrium processes in clusters of galaxies. On a small scale, we studied observationally a cooling core cluster with AGN feedback; on a large scale, we studied theoretically non-equilibrium processes in cluster accretion shock regions.

XMM-Newton and Chandra observations of Abell 2626 are presented. We do not find an obvious correlation between the radio bars and the X-ray image. We suggest that the two symmetric radio bars can be explained by two precessing jets ejected from an AGN. We argue that Abell 2626 is preferentially accreting subclusters and groups from large-scale structure filaments. We also find evidence that the S0 galaxy IC 5337 is infalling toward the cluster center from the west.

Electrons and ions which pass through an accretion shock may not achieve equipartition due to the long Coulomb collisional timescale in the low density environment. We have studied the non-equipartition effects in the outer regions of relaxed clusters using one-dimensional hydrodynamic simulations. We find that in the $\Lambda \mathrm{CDM}$ universe, non-equipartition effects are larger for clusters at higher redshifts and with higher masses. We also present in detail the X-ray and Sunyaev-Zel'dovich (SZ) signatures of non-equipartition.

The impact of non-equipartition effects on cosmological parameter estimation from SZ surveys is studied. We find that non-equipartition can bias the cosmological parameters $\Omega_{M}, \sigma_{8}$, and the dark energy equation of state parameter $w$ by a few percent. In particular, non-equipartition effects can introduce an apparent evolution in $w$.
\end{abstract}

Non-equilibrium ionization (NEI) effects in the outer regions of relaxed clusters are also studied. We find that NEI effects do not depend on cluster mass but in- 
crease as redshift decreases, which can be understood by self-similar arguments. The most prominent NEI signature we found is the O VII and O VIII line ratio, which is different by more than an order of magnitude for NEI and collisional ionization equilibrium (CIE) models at radii beyond half of the shock radius. We conclude that a $\sim 130-220 \mathrm{ksec}$ exposure on a moderate-redshift, massive regular cluster with the International X-ray Observatory (IXO) should provide a strong test for the NEI model by measuring this line ratio. 


\section{Acknowledgements}

I owe my deepest gratitude to my thesis advisor, Craig Sarazin, who is in no doubt a great scientist and mentor. He has shown me how to do science both rigorously and seriously, although I feel I have not learned enough from him. Even though he is always very busy with his own research, administrative work, and advising many of his students and postdocs, he has always been willing to spend a significant amount of time in discussing research or chatting with me. Every time we have met, I have been inspired a lot by his great scientific insights and amused by his humor. I must

also thank Craig for his patience in correcting the numerous English (and logical) errors I made in my writings. In addition, I enjoyed going to parties in his house, and I will never forget the delicious dishes he prepared for us. It is my great pleasure and honor to know Craig.

I would like to thank Massimiliano Bonamente, John Hawley, Harry Thacker, and Mark Whittle for being on my thesis committee. John Hawley and Mark Whittle have attended numerous thesis committee meetings with me in the past years, and their suggestions have helped me to sharpen my thesis greatly. I thank Massimiliano Bonamente and Harry Thacker for spending their valuable time to read my thesis and for their very useful discussions.

I must also thank my collaborators and colleagues, who have contributed to or have given me valuable suggestions about my thesis. I must again thank Craig Sarazin, who has spent large amounts of time giving me directions and suggestions for all of the projects in which we have collaborated. I have learned a lot from Elizabeth Blanton and Thomas Reiprich during our collaboration. Since I started to work on theoretical projects, Avi Loeb has spent a significant amount of time discussing ideas with me, and I must thank him deeply. I also thank Daniel Wik, who has worked with me on 
the cosmological topic. I have learned a lot about radio astronomy from Cheng-Yu Kuo and Brian Mason. I also enjoyed working with Li Ji on the non-equilibrium ionization problem. I should also thank Heinz Andernach, Kazimierz Borkowski, Marios Chatzikos, Tracy Clarke, Jim Condon, Yutaka Fujita, Myriam Gitti, Craig Heinke, Amalia Hicks, Adrienne Juett, Joe Mohr, Scott Randall, Gregory Sivakoff, Ming Sun, Todd Tripp, and Daniel Wang for their useful discussions.

I have to thank the Graduate Student Advisors, Zhi-Yun Li and Steve Majewski, who have taken care of us in the department. I would also like to thank Kiriaki Xiluri and Howard Powell, who helped me with the computers. In particular, Howard has helped to recover some very important data and hence saved my life. My thanks also go out to the very helpful staff members, Janice Dean, Jackie Harding, and Barbara Johnson. Life in the department would be disorganized without them.

Of course, I have to thank all of the great teachers and graduate students in the Astronomy Department and also the Physics Department at the University of Virginia. I will not forget the time I spent listening to lectures from professors, doing homework and studying for qualifying exams with classmates, playing soccer and softball, going to picnics, enjoying the East Asian Student Lunches and Dinners, and doing lots of other things. Countless helpful people were encountered during conferences and seminars. I could not list their names one by one, but I would like to say thank you here.

During the course of this work at the University of Virginia, I was supported by the Department of Astronomy and also by various grants from the National Aeronautics and Space Administration (NASA). Partial support was also provided by the F. H. Levinson Fund, the Office of the Vice President for Research at the University of Virginia, and the Society of Fellows Travel Fellowship of the Graduate School of 
Arts and Sciences at the University of Virginia. Modifications of the thesis and the write-up of the acknowledgement were done when I was at the University of Alabama at Tuscaloosa, and I thank Jimmy Irwin for arranging the necessary computing resources.

I would like to voice my gratitude to my master advisor, Ming-Chung Chu, at the Chinese University of Hong Kong for his guidance and continuous support during these years. I also want to thank Kwong Sang Cheng and Jason Pun at Hong Kong University for their encouragement during my academic career.

Special thank goes to Doctor D. who helped proofread this acknowledgement.

Finally, and most importantly, this work would not have been possible without the endless support and encouragement from my parents and my love. I dedicate this thesis to them.

August, 2010 


\section{Table of contents}

Abstract

Acknowledgements $\quad$ v

List of Figures $\quad$ xx

List of Tables $\quad$ xxi

1 General Introduction 1

1.1 Clusters of Galaxies . . . . . . . . . . . . . . . . . . . . . . . . 1

1.2 Importance to Cosmology . . . . . . . . . . . . . . . . . . 4

1.3 Beyond Equilibrium Physics . . . . . . . . . . . . . 5

1.3.1 Feedback from Central AGN: the Case of Abell $2626 \ldots \ldots$. . . 7

1.3.2 Non-Equilibrium Processes in the Outer Regions of Clusters . 9

1.4 Thesis Outline . . . . . . . . . . . . . . . . . . . . . 10

2 XMM-Newton and Chandra Observations of Abell 2626:

Interacting Radio Jets and Cooling Core with Jet Precession? 11

2.1 Introduction . . . . . . . . . . . . . . . . . . . . . . . 11

2.2 Observations and Data Reduction . . . . . . . . . . . . 16

2.2 .1 XMM-Newton Data . . . . . . . . . . . . . . . . . 16

2.2 .2 Chandra Data . . . . . . . . . . . . . . . . . . . . . 17

2.3 X-Ray Image Analysis . . . . . . . . . . . . . . . . . . . . . . . . . . 19

2.3 .1 X-ray Morphology . . . . . . . . . . . . . . . . . . 19

2.3 .2 Source Detection . . . . . . . . . . . . . . . . . . . . . . 23

2.3.3 Surface Brightness Profile . . . . . . . . . . . . . . 24

2.3 .4 Residual Images . . . . . . . . . . . . . . . . . . . . . . 26

2.4 Spectral Analysis . . . . . . . . . . . . . . . . . . . . . . . . . . . . . . . 28

2.4 .1 Temperature Profile . . . . . . . . . . . . . . . 28

2.4.2 Central X-ray Spectrum and Cooling Rate . . . . . . . . . . 34

2.4.3 Density, Pressure and Entropy Profiles . . . . . . . . . . . . 35

2.4.4 Cooling and Thermal Conduction Time Scales . . . . . . . . 38

2.4 .5 Mass Profiles . . . . . . . . . . . . . . . . . . . . 41 
2.4 .6 Hardness Ratio Maps . . . . . . . . . . . . . . . . . . . . . 42

2.4.7 Possible X-ray AGNs in IC 5338 and IC $5337 \ldots \ldots . \ldots . .45$

2.5 Kinematics of the S0 Galaxy IC $5337 \ldots \ldots \ldots \ldots \ldots$

2.6 Cooling Flow Interaction with the Central Radio Source? . . . . . . . 50

2.7 Conclusions . . . . . . . . . . . . . . . . . . . . . . . 55

Appendix A Density Deprojection . . . . . . . . . . . . . . . . 60

Appendix B Cooling Time Estimation . . . . . . . . . . . . . 62

3 Effects of the Non-Equipartition of Electrons and Ions in the Outskirts of Relaxed Galaxy Clusters $\quad 79$

3.1 Introduction . . . . . . . . . . . . . . . . . . . . . . . 79

3.2 Hydrodynamic Model . . . . . . . . . . . . . . . . . . . . . . 86

3.2 .1 Simulation Code . . . . . . . . . . . . . . . . 87

3.2.2 Boundary Conditions and Computational Domain . . . . . . 88

3.2.3 Initial Conditions for the Realistic NFW-Dark Energy Cluster Models . . . . . . . . . . . . . . . . . . . 90 90

3.2.4 Concentration Parameters . . . . . . . . . . . . . . . . 94

3.2.5 Self-consistent Evolution of NFW Potential Including Accreted Mass . . . . . . . . . . . . . . . . . . . . . 95

3.2.6 Dark Energy Implementation _ . . . . . . . . . . . . . . 96

3.3 Electron Heating within a Cluster . . . . . . . . . . . . . . . 97

3.3.1 General Picture of Shock Heating and the Equilibration between Electrons and Ions Afterward . . . . . . . . . . . . . 97

3.3.2 Electron Heating within the Thin Shock Front . . . . . . . . . 101

3.3.3 Coulomb Equilibration After Shock Heating . . . . . . . . . 103

3.3.4 Steps to Calculate the $T_{e} / \bar{T}$ Evolution . . . . . . . . . . 104

3.4 Test Models: Self-similar Models . . . . . . . . . . . . . . . . . 105

3.4.1 Collisionless Dark Matter Dominated Accretion Model . . . . 106

3.4.2 Collisional Gas Dominated Accretion Model . . . . . . . . . 108

3.4.3 Self-similar Non-equipartition Model . . . . . . . . . . . . . . 109

3.5 Dynamics for Realistic NFW-Dark Energy Models . . . . . . . . . . . 109

3.6 Observables for Realistic NFW-Dark Energy Models . . . . . . . . . . 110

3.6.1 Definition of X-ray Observables . . . . . . . . . . . . . 110

3.6.2 Definition of SZ Observables . . . . . . . . . . . . . . . . 112

3.6.3 Results for the Temperature Profiles and the X-ray Observables 114

3.6.4 Results for the SZ Temperature Decrement . . . . . . . . . . . 119

3.6.5 Results for the Integrated SZ Biases, $Y_{\text {non-eq }} / Y_{\text {eq }}$, and its Evolution . . . . . . . . . . . . . . . . . . . 122

3.7 Discussions and Conclusions . . . . . . . . . . . . . . . . . . . 125

Appendix A Expression for Electron Heating within the Shock . . . . . . 130 
4 The Impact of Non-Equipartition on Cosmological Parameter Estimation from Sunyaev-Zel'dovich Surveys $\mathbf{1 4 5}$

4.1 Introduction . . . . . . . . . . . . . . . . . . 145

4.2 SZ Versus Mass Correlation . . . . . . . . . . . . . . . . . 149

4.3 Effects of Non-Equipartition on SZ Surveys . . . . . . . . . . . . . . . 152

4.4 Effects of Non-Equipartition on Cosmological Parameter Estimation from SZ Surveys . . . . . . . . . . . . . . . . . . . . 155

4.4.1 Systematic Uncertainties Introduced by Non-Equipartition . . 156

4.4.2 Fitting Procedures and Results . . . . . . . . . . . . . . . 157

4.5 Discussion and Conclusions . . . . . . . . . . . . . . . . . 160

5 X-ray Signatures of Non-Equilibrium Ionization Effects in Galaxy $\begin{array}{lr}\text { Cluster Accretion Shock Regions } & 171\end{array}$

5.1 Introduction . . . . . . . . . . . . . . . . . . . . 171

5.2 Physical Models and Calculational Techniques . . . . . . . . . . . . 175

5.2.1 Hydrodynamic Models and Electron Temperature Structures . 175

5.2 .2 Ionization Calculations . . . . . . . . . . . . . . . . . . 177

5.2.3 X-ray Emission Calculations . . . . . . . . . . . . . . . . . . . 179

5.3 Dependence of Non-Equilibrium Ionization on Cluster Mass and Redshift180

5.4 Non-Equilibrium Ionization Signatures . . . . . . . . . . . . . . 185

5.4.1 Models Used to Calculate Spectra . . . . . . . . . . . . . . 185

5.4 .2 X-ray Spectra . . . . . . . . . . . . . . . . 186

5.4 .3 Surface Brightness Profiles . . . . . . . . . . . . . . . . 187

5.4 .4 O VII and O VIII Line Ratio . . . . . . . . . . . . . . . . . 188

5.5 Detectability of $\mathrm{O}$ VII and O VIII Lines with IXO and Testing the

Non-Equilibrium Ionization Effect . . . . . . . . . . . . . . . . . 192

5.5.1 Backgrounds . . . . . . . . . . . . . . . . . 193

5.5.2 Signal-to-Noise Ratio . . . . . . . . . . . . . . . . . . 195

5.6 Discussions and Conclusions . . . . . . . . . . . . . . . . . 198

6 Conclusions and Future Directions for Research 216

6.1 Summary . . . . . . . . . . . . . . . . . 217

6.2 Future Directions for Research . . . . . . . . . . . . . . . . . . 221

A Can Non-Equipartition at Cluster Accretion Shocks be Detected with ALMA? $\quad 228$ 


\section{List of Figures}

2.1 Background-subtracted, exposure-corrected, adaptively smoothed mosaic of the XMM-Newton EPIC MOS1 and MOS2 images in the 0.3-10 $\mathrm{keV}$ band. The image was smoothed to a signal-to-noise ratio of 3 . The units for the color scale are counts per binned pixel, where each binned pixel has a size of $4^{\prime \prime} .1 \times 4^{\prime \prime}$. 1 . The arrows on the left and right indicate the $\mathrm{cD}$ galaxy IC 5338 and the S0 galaxy IC 5337, respectively. Two outer extended emission regions are indicated with white circles. It should be noted that there is a chip gap at about 5.5 from the center which makes the northeast extended X-ray source appear to be more distinct than it actually is. . . . . . . . . . . . . . .

2.2 Left panel: Background subtracted, exposure-corrected, adaptively smoothed Chandra image of the central 3.5 $\times 2$ '.4 region of the cluster in the $0.3-10 \mathrm{keV}$ band. The image was smoothed to a signal-to-noise ratio of 3. The Chandra image shows the details of the inner region of the cooling core. The white solid lines are $1.5 \mathrm{GHz}$ C-array radio contours showing the mini-halo. The green solid lines are $1.5 \mathrm{GHz}$ B-array radio contours showing the radio bar structures. Both radio contours were taken from Gitti et al. (2004). The arrows on the left and right indicate the cD galaxy IC 5338 and the S0 galaxy IC 5337, respectively. Right panel: Background subtracted, exposure-corrected, adaptively smoothed Chandra image centered on the S0 galaxy IC 5337. The image was smoothed to a signal-to-noise ratio of 4 , with an intensity scale chosen to show the bow-shock-like shape. The southern source (red ellipse) was removed and replaced by the average surrounding X-ray intensity before adaptively smoothing. The green contours are from the VLA $1.5 \mathrm{GHz}$ B-array radio image taken from Gitti et al. (2004), with the lowest contour level being a signal-to-noise ratio of 3 . 
2.3 Images of the two nuclei of the cD galaxy IC 5338 in Abell 2626. All images show the same field of view of $30^{\prime \prime} \times 30^{\prime \prime}$. Upper left panel: Optical image from HST archive. The image was taken with WFPC2 using the F555W filter. The two green circles, included in the other panels, are centered at the two optical nuclei observed by HST. Upper right panel: Background-subtracted, exposure-corrected, adaptively smoothed Chandra image in $0.3-10 \mathrm{keV}$ band. The image was smoothed to a signal-to-noise ratio of 3 . The color represents the X-ray intensity from high (white yellow) to low (dark blue). Lower left panel: Raw Chandra image in the soft $(0.3-2 \mathrm{keV})$ band. Two intensity peaks can be identified. Lower right panel: Raw Chandra image in the hard (2$10 \mathrm{keV}$ ) band. Only the southwest nucleus corresponds to the peak in the hard band. . . . . . . . . . . . . . . . . . . . . . .

2.4 Optical image from the Digital Sky Survey showing the 9 XMM-Newton X-ray sources with identifications in NED (Table 2.1). The red circles are centered on the X-ray positions determined with XMM-Newton. There are green circles centered on the optical positions from NED, but they overlap so completely with the red circles that they are barely visible. NED objects are considered possible identifications if they are within $20^{\prime \prime}$ of an X-ray source. In fact all optical counterparts identified are within $11^{\prime \prime}$. . . . . . . . . . . . . . . . . . . .

2.5 Optical image from the Digital Sky Survey showing the positions (red circles) of the 14 X-ray point sources detected with Chandra (Table 2.2) . . . . . . . . . . . . . . . . . . . . . .

2.6 Upper panel: Azimuthally averaged X-ray surface brightness profile from the XMM-Newton MOS1 and MOS2 observations of Abell 2626. The squares are the X-ray data, while the solid line is the fit to a double $\beta$ model. The tiny error bars within the squares on the surface brightness are too small to be easily seen. Lower panel: The residuals to the best fit double beta model, in unit of $\sigma$. The horizontal error bars show the widths of the bins used to accumulate the counts. The vertical error bars are at the $1 \sigma$ level. . . . . . . . . . . . . . 
2.7 Left panel: Residual map from the XMM-Newton MOS1 and MOS2 observations of Abell 2626, generated from a background-subtracted image. Yellow represents the largest excess, while black is the greatest deficit. Right panel: Residual map from the Chandra observation of Abell 2626, generated from a background-subtracted image. Yellow represents the largest excess, while black is the greatest deficit. There is an excess coincident with the center of the central $\mathrm{cD}$ galaxy, which is partly surrounded by a trough of reduced X-ray surface brightness at a radius of about $15^{\prime \prime}$. There are two localized regions of excess south and north of the central peak, which are indicated with green circles. There is an extended deficit about $50^{\prime \prime}$ west of the center, which is indicated with a white polygon. An extended region of excess emission is seen about $110^{\prime \prime} \mathrm{SW}$ of the center, which is indicated with a green polygon. . . . . . . . . . . . . . . . . .

2.8 Residual map from Chandra observation of the central 3.5 $\times 2$ '.4 region of Abell 2626, generated from a background-subtracted image. The color scale from yellow to red to blue to black represents the residuals, running from excess to deficit. The white solid lines are $1.5 \mathrm{GHz} \mathrm{C}$ array radio contours showing the mini-halo. The green solid lines are 1.5 GHz B-array radio contours showing the radio bar structures. Both radio contours were taken from Gitti et al. (2004). The arrows on the left and right indicate the cD galaxy IC 5338 and the S0 galaxy IC 5337, respectively. . . . . . . . . . . . . . . . .

2.9 Upper Panel: Profile of the azimuthally averaged, projected temperature $T$ of Abell 2626. The XMM-Newton (Chandra) data are shown as solid (open) circles. Middle Panel: Profile of the azimuthally averaged, deprojected temperature $T$ of Abell 2626. The XMM-Newton (Chandra) data are shown as solid (open) circles. The black crosses are the projected temperatures from the XMM-Newton data in the upper panel for comparison (without error bars for clarity). In both the upper and middle panels, the error bars are at the $90 \%$ confidence level. Lower Panel: Crosses are the temperatures calculated from interpolation of the original XMM-Newton deprojected temperature profile; this interpolated profile was used for the pressure/entropy/time scales deprojection and the mass profile analysis in Sections 2.4.3, 2.4.4, \& 2.4.5. The interpolated temperature profile for the Chandra data analysis is similar but not shown for clarity. The errors are assigned as 5 (20)\% at radii of smaller (larger) than 250" to include possible systematic errors. The solid circles are the original XMM-Newton measured deprojected temperatures as shown in the middle panel, without error bars for clarity. . . . . . . . . . . . . . . . . . . 
2.10 Profile of the azimuthally averaged, deprojected temperature $T$ of Abell 2626 with different XMM-Newton backgrounds. The black diamonds give our adopted XMM-Newton temperature profile (the black solid circles in the second panel of Figure 2.9). The other temperature profiles are generated by: 1a) (blue diamonds) increasing the blank-sky background normalizations by 10\% (20\%) for MOS (PN); 1b) (green diamonds) decreasing the blank-sky background normalizations by $10 \%$ (20\%) for MOS (PN); 2) (red crosses) using the blank-sky backgrounds without renormalization (no error bars for clarity); 3) (magenta triangles) using the MOS1+MOS2 spectra only (data shifted to the right for clarity); 4) (cyan triangles) using the PN spectra only (data shifted to the left for clarity); 5) (orange crosses) adding an extra $0.2 \mathrm{keV}$ MEKAL component to represent variations in the soft Galactic component (normalization free and allowed to be negative in each annulus, data shifted to the left for clarity). The error bars are at the $90 \%$

2.11 Upper Panel: Azimuthally averaged electron density profile of Abell 2626. The XMM-Newton (Chandra) data are shown as black solid (red dashed) symbols. For clarity, we have also multiplied the Chandra data and error bars by a factor of 2 (green solid symbols). The error bars are at the $1 \sigma$ level. Middle Panel: Azimuthally averaged pressure profile, with the same notation as the upper panel. Lower Panel: Azimuthally averaged entropy profile, with the same notation as the upper panel.

2.12 Upper Panel: Cooling and thermal conduction time scales in Abell 2626 obtained from the XMM-Newton data. The cooling times are the plus symbols with horizontal bars showing the annular regions sampled. The conduction time scales derived directly from individual data points are the crosses with larger vertical error bars. The dots are conduction time scales calculated by interpolating the temperature profile of the cluster (see text in Section 2.4.4). The radial regions for the conduction time are not given for clarity. The error bars are at the $1 \sigma$ level. Lower Panel: Same as the upper panel, but with the time scales calculated from the Chandra data. . . . . . . . . . . . . . . . . .

2.13 Upper panel is the interior mass profile $[M(<r)]$, of Abell 2626 obtained from XMM-Newton data, while the lower panel is from Chandra data. The total mass is shown with diamond symbols, while the gas mass points are circles. The error bars on the masses are calculated using propagation of errors at $1 \sigma$ level. The error bars on the gas mass are plotted, but are smaller than the circle symbols. . . . . . . . . 
2.14 Hardness ratio $(H R)$ map of Abell 2626 from the XMM-Newton observation. Background was subtracted from the images combined to calculate the hardness ratio. The color scale ranges from black $(H R \sim 0)$ to blue $(H R \sim 0.1)$ to red $(H R \sim 0.3)$ to yellow $(H R \sim 0.5)$ to white $(H R \sim 2.5)$, showing increasingly hard emission. The green solid lines are contours from XMM-Newton residual map (left panel of Figure 2.7) showing positive residuals (excess emission). The white circles are the regions of extended X-ray emissions to the northeast and southwest. The cyan circle has a radius of $240^{\prime \prime}$ corresponding to the jump in the temperature profile. . . . . . . . . . . . . . . .

2.15 Left panel: Central portion $\left(4^{\prime} \cdot 6 \times 4^{\prime} \cdot 7\right)$ of the XMM-Newton hardness ratio map (Figure 2.14). The color scale is similar to that in Figure 2.14, but with the hardness ratio extremes being $\sim 0.2$ and 0.35 (dark blue to white yellow). The green solid lines are contours from the Chandra residual map (right panel of Figure 2.7) showing the excess emission. Right panel: Hardness ratio map from the Chandra data, for the same regions and with the same contours as the left panel. Background was subtracted from the images combined to calculate the hardness ratio. The color scale is similar to that in Figure 2.14, but with the hardness ratios in the range from $\sim 0.1$ to 1.5 (dark blue to white yellow). The cD galaxy IC 5338 and the S0 galaxy IC 5337 are located at $(23: 36: 31$, $+21: 08: 47)$ and $(23: 36: 25,+21: 09: 03)$, respectively. . . . . . . . .

2.16 Central region $(3.5 \times 2 ! 4)$ of the hardness ratio map from the Chandra observation. Background was subtracted from the images combined to calculate the hardness ratio. The color scale is roughly the same as in the right panel of Figure 2.15. The white solid lines are $1.5 \mathrm{GHz}$ C-array radio contours showing the mini-halo. The green solid lines are $1.5 \mathrm{GHz}$ B-array radio contours showing the radio bar structures. Both radio contours were taken from Gitti et al. (2004). The arrows on the left and right indicate the $\mathrm{cD}$ galaxy IC 5338 and the S0 galaxy IC 5337, respectively. . . . . . . . . . . . . . .

3.1 Gas velocity (upper panel), density (middle panel), and pressure (lower panel) profiles of our simulated cluster for the self-similar collisionless dark matter dominated accretion model in the Einstein-de Sitter universe at four different redshifts. The model has a dark matter mass accreted within $R_{178}$ of $M_{178}=10^{15} M_{\odot}$ at $z=0$. The insets in the upper and middle panels show the large radius behavior of the gas, with the velocity profile shown for $z=0$ only. The circles on the inset of the velocity profile give the Hubble flow velocity at $z=0$. We set $\Omega_{b}=0.05$ in this model. 
3.2 Scaled gas velocity (upper panel), density (middle panel), and pressure (lower panel) profiles of our simulated cluster. The hydrodynamic model is the same as in Figure 3.1. All four lines lie almost on top of one another in the figures. Circles show the corresponding analytic solutions.

3.3 Similar to Figure 3.2 but for the self-similar collisional gas dominated accretion model in the Einstein-de Sitter universe. The model has a gas mass accreted within $R_{178}$ of $M_{178}=1.18 \times 10^{15} M_{\odot}$ at $z=0$. . .

3.4 Ratio of the electron and average thermal dynamic temperature, $\tau$, as a function of scaled radius $r / R_{\text {sh }}$ for clusters of the self-similar collisionless dark matter dominated accretion model in the Einstein-de Sitter universe at $z=0$. The upper and lower solid lines are the analytic solutions for cluster masses with $M_{178}=5.26 \times 10^{14} M_{\odot}$ and $M_{178}=1.05 \times 10^{15} M_{\odot}$, respectively. The open circles on the corresponding lines are our simulated results. We assume $\Omega_{b}=0.05$ here.

3.5 Gas velocity (upper panel), density (middle panel), and pressure (lower panel) profiles of our simulated cluster for the realistic NFW-dark energy model in the standard $\Lambda \mathrm{CDM}$ universe at four different redshifts. The model has total mass accreted within $R_{\mathrm{sh}}$ of $M_{\mathrm{sh}}=1.53 \times 10^{15} M_{\odot}$ at $z=0$. The insets show the large radius behavior of the gas, with the velocity profile shown for $z=0$ only. The circles on the inset of the velocity profile give the Hubble flow velocity at $z=0 . \quad . .$. .

3.6 Average thermodynamic temperature profiles of our simulated realistic NFW-dark energy model cluster. The hydrodynamic model is the same as in Figure 3.5.

3.7 Average thermodynamic temperature profiles of our simulated selfsimilar dark matter dominated cluster. The hydrodynamic model is the same as in Figure 3.1.

3.8 Ratio of electron and average thermodynamic temperatures, $\tau \equiv T_{e} / \bar{T}$, as a function of radius at $z=0$. The hydrodynamic model is the same as in Figure 3.5. The models with shock heating efficiency $\beta=1 / 1800$ and 0.5 are shown in solid and dashed lines, respectively. . . . . . . .

3.9 Projected X-ray spectroscopic-like temperature profiles for cluster models at $z=0$. The hydrodynamic model is the same as in Figure 3.5. The non-equipartition model with $\beta=1 / 1800$ is shown in solid line, while the equipartition model is shown in dashed line. We assume $Z=0.3 Z_{\odot}$ here.

3.10 Ratio of the projected X-ray spectroscopic-like temperature profiles of the non-equipartition (with $\beta=1 / 1800$ and 0.5 ) and the equipartition models at $z=0$. Models with $Z=0.1$ and $0.3 Z_{\odot}$ are presented. The hydrodynamic model is the same as in Figure 3.5. 
3.11 Ratio of the projected X-ray spectroscopic-like temperature of the non-equipartition with $\beta=1 / 1800$ and the equipartition models as a function of $r / R_{\mathrm{sh}}$ at $z=0$ for different cluster masses. The hydrodynamic models are similar to those in Figure 3.5, but with $M_{\mathrm{sh}}=$ $7.65,15.3$, and $30.6 \times 10^{14} M_{\odot}$ at $z=0$. We assume $Z=0.3 Z_{\odot}$ here.

3.12 Surface brightness profiles for various energy bands for the non- equipartition (solid lines) and equipartition (dashed lines) cluster models at $z=0$. Models are the same as in Figure 3.9. . . . . . . . . . . .

3.13 Ratios $S_{\text {non-eq }} / S_{\text {eq }}$ as a function of radius. Models are the same as in Figure 3.12. The ratio of the bolometric surface brightness near the shock radius reaches $\sim 35$ (outside the scale of the figure).

$3.14 \mathrm{SZ}$ temperature decrement profiles, $-\Delta T_{\mathrm{SZE}}$, of our simulated cluster at four different redshifts. The non-equipartition models $(\beta=1 / 1800)$ are shown in solid lines, while the equipartition models are shown in dashed lines. The hydrodynamic model is the same as in Figure 3.5.

3.15 Difference $\delta \Delta T_{\mathrm{SZE}}$ between the SZ temperature decrements of the equipartition and the non-equipartition models $(\beta=1 / 1800)$ at four different redshifts. Models are the same as in Figure 3.14. . . . . . . . . . . . 142

3.16 Ratio $y_{\text {non-eq }} / y_{\text {eq }}$ between the Comptonization parameters of the equipartition and the non-equipartition models $(\beta=1 / 1800)$ at four different redshifts. Models are the same as in Figure 3.14.

3.17 Same as Figure 3.14 but with $M_{\mathrm{sh}}(z=0)=3.06 \times 10^{15} M_{\odot}$ for the cluster model.

3.18 Same as Figure 3.15 but with $M_{\mathrm{sh}}(z=0)=3.06 \times 10^{15} M_{\odot}$ for the cluster model.

3.19 Integrated SZ biases, $Y_{\text {non-eq }} / Y_{\text {eq }}$, as a function of $M_{\text {sh }}$ for both our simulated realistic NFW model in the $\Lambda$ CDM universe (thin lines) and the numerical simulated self-similar model in the Einstein-de Sitter universe (thick lines) at four different redshifts. We assume $f_{\text {gas }}=0.17$ for models in the Einstein-de Sitter universe. The four lines for the self-similar model lie almost along the same line which cannot be easily distinguished on the graph.

4.1 Top panel: Integrated Comptonization parameter $Y$ versus mass at $z=0$ for the equipartition model used in this chapter. Bottom panel: Integrated $Y$ bias, $\left(Y_{\text {non-eq }} / Y_{\text {eq }}\right)_{\mathrm{ws}}$, versus mass at $z=0$ and $2 . .$.

4.2 Top panel: Integrated $Y$ functions of the non-equipartition (solid lines) and the equipartition (dotted lines) models in the standard $\Lambda \mathrm{CDM}$ universe. Solid dots indicate the values of $Y_{0}$ such that $\int_{Y_{0}}^{\infty} \int_{z_{l}}^{z_{u}} n_{\mathrm{PS}}(Y, z)$ $d Y(d V / d z) d z=1$ for the equipartition model. Bottom panel: Ratios between the non-equipartition and equipartition $Y$ functions. 
4.3 Non-equipartition $Y$ functions (crosses) and the best fitted $Y$ functions (lines) at different redshifts are plotted on each figure in row 1, 3, and 5 for Case 1, 2, and 3, respectively. Residuals in the log of the nonequipartition $Y$ functions are plotted under the corresponding figures. Figures in Column 1 correspond to models with dark energy parameters frozen at $w=1$. Figures in Column 2 correspond to models with $w$ fitted as constant parameters ( $w_{1}$ frozen at zero). Figures in Column 3 correspond to models with $w$ allowed to vary with redshift. Redshifts at $z=0,0.5,1$, and 2 are shown in solid, dash-dotted, dashed, and dotted lines, respectively. . . . . . . . . . . . . . .

5.1 Ionization timescale parameter $\tau$ (Equation (5.2)) versus radius $r$ scaled to the cluster shock radius $R_{\text {sh }}$ for cluster models with total accreted masses of $M_{\mathrm{sh}}=0.77$ (dashed line), 1.53 (solid line), and 3.06 (dotted line) $\times 10^{15} M_{\odot}$ at a redshift of $z=0$. The shock radii for the three clusters from small to high mass are $R_{\mathrm{sh}}=3.31,4.22$, and $5.41 \mathrm{Mpc}$, respectively. All three lines lie almost on top of one another.

5.2 Dimensionless ionization parameter $C_{i}\left(T_{e}\right) \tau$ for O VII versus the scaled radius $\left(r / R_{\mathrm{sh}}\right)$ for clusters with total accreted masses of $M_{\mathrm{sh}}=0.77$ (dashed line), 1.53 (solid line), and 3.06 (dotted line) $\times 10^{15} M_{\odot}$ at a redshift of $z=0$. Models with $\beta=1 / 1800$ (non-equipartition) and $\beta=1$ (equipartition) are shown in thick and thin lines, respectively.

5.3 Dimensionless ionization parameter $C_{i}\left(T_{e}\right) \tau$ for O VII versus the scaled radius $\left(r / R_{\mathrm{sh}}\right)$ for clusters at different redshifts. The cluster model with a total accreted mass $M_{\mathrm{sh}}=1.53 \times 10^{15} M_{\odot}$ at $z=0$ is used. Models with $\beta=1 / 1800$ (non-equipartition) and $\beta=1$ (equipartition) are shown in thick and thin lines, respectively. . . . . . . . . . . .

5.4 Non-equilibrium ionization bias versus the scaled radius $\left(r / R_{\mathrm{sh}}\right)$ for clusters with total accreted masses of $M_{\mathrm{sh}}=0.77$ (dashed line), 1.53 (solid line), and 3.06 (dotted line) $\times 10^{15} M_{\odot}$ at a redshift of $z=0$. Models with $\beta=1 / 1800$ (non-equipartition) and $\beta=1$ (equipartition) are shown in thick and thin lines, respectively. . . . . . . . . . . . 208

5.5 Non-equilibrium ionization bias versus the scaled radius $\left(r / R_{\mathrm{sh}}\right)$ for clusters at different redshifts. The cluster model with a total accreted mass $M_{\text {sh }}=1.53 \times 10^{15} M_{\odot}$ at $z=0$ is used. Models with $\beta=1 / 1800$ (non-equipartition) and $\beta=1$ (equipartition) are shown in thick and thin lines, respectively. . . . . . . . . . . . . . . . 
5.6 Projected rest frame spectra for the cluster model with an accreted mass of $M_{\mathrm{sh}}=1.53 \times 10^{15} M_{\odot}$ at $z=0$ are shown at two projected radii (left: $2 \mathrm{Mpc}$; right $3.5 \mathrm{Mpc}$ ). Upper panels: the NEI model with $\beta=1 / 1800$ and non-equipartition of electrons and ions. Lower panels: projected rest frame spectra for the CIE model with the same $\beta$ and non-equipartition. . . . . . . . . . . . . . .

5.7 Upper left panel: rest frame projected surface brightness profiles for different energy bands for the NEI model with $\beta=1 / 1800$ (thick lines) and the CIE-Non-Eq model (thin lines). Upper right panel: rest frame projected surface brightness profiles for the NEI model with $\beta=1 / 1800$ (thick lines) and the CIE-Eq model (thin lines). Lower left panel: ratios of the surface brightness profiles $S_{\mathrm{NEI}} / S_{\mathrm{CIE}-\mathrm{Non} \text {-Eq }}$. Lower right panel: ratios of the surface brightness profiles $S_{\mathrm{NEI}} / S_{\mathrm{CIE}-\mathrm{Eq}} \cdot$. .

5.8 Upper panel: projected rest frame spectra for the NEI model with $\beta=1 / 1800$. Middle panel: projected rest frame spectra for the NEI model with $\beta=0.5$. Lower panel: projected rest frame spectra for the CIE-Non-Eq model with $\beta=1 / 1800$. All spectra are for the $M_{\mathrm{sh}}=1.53 \times 10^{15} M_{\odot}$ at $z=0$, and are for a projected radius of $r=2 \mathrm{Mpc}$. The spectra are binned with $\Delta E=0.1 \mathrm{eV} . \ldots$.

5.9 Surface brightness of the O VII and O VIII lines for the NEI model with $\beta=1 / 1800$ as a function of projected radius. The continuum emission within the narrow energy bands covering the lines is also shown. . .

5.10 Line ratios $S(\mathrm{O}$ VIII) $/ S(\mathrm{O}$ VII) for the NEI models with $\beta=1 / 1800$ (solid), 0.5 (dash-dotted), and 1.0 (dash-dot-dotted). Line ratios for the CIE-Non-Eq (dashed) and CIE-Eq (dotted) models are also shown. 214

5.11 Left (middle) panels show the signal-to-noise ratios for the O VII triplets (O VIII doublets) expected to be detected by IXO. The right panels show the signal-to-noise ratios for the O VII and O VIII line ratios. The upper panels correspond to the XMSC, and the lower panels correspond to the XMSF. Different signal and noise levels are assumed for the different models. The legends for the lines are the same for all panels, but are separated into two panels due to the space limitation.

A.1 (Left panel) Central region of the SZ image for the non-equipartition model in angular (rather than $u v$ ) coordinates. The edge of the circle indicates the shock radius which is about $180^{\prime \prime}$ from the cluster center. The intensity bar is in units of K. (Right panel) Same as the left panel, but the primary beam response with a Gaussian beam with FWHM $=2.5^{\prime}$ has been applied. The primary beam is centered at the shock radius on the right hand side of the cluster. . . . . . . . . . . .

A.2 Radial profiles along the horizontal axis of the left panel (dashed line) and the right panel (solid line) in Figure A.1. . . . . . . . . . . . . . 233 
A.3 (Left panel) Real part of the visibility function for the image in the right panel in Figure A.1. (Right panel) Imaginary part of the visibility function. The visibility function has been normalized by the primary beam area, so that the intensity bars in both panels are in unit $\mathrm{K}$. .

A.4 Visibility functions for positive $u$ at $v=0$. The solid line is the amplitude, the dashed line is the real part, and the dotted line is the negative of the real part. The imaginary part is not plotted on the figure. . .

A.5 $u v$ coverage of the most compact configuration of ALMA with $\sqrt{u^{2}+v^{2}}$

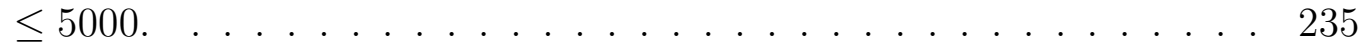

A.6 (Top panel) Simulated data projected along $u$ for one of the 10,000 Monte Carlo simulations. Errors are included in the simulated real and imaginary visibility data. The best fitted values are also shown. (Bottom panel) Same as the top panel, but for visibility data projected along $v$. . . . . . . . . . . . . . . . . . . . . . .

A.7 Residuals for positive $u$ along $v=0$ for one of the 10,000 Monte Carlo simulations. The residual is defined as the best fitted model minus the raw input data (Figure A.3). Solid line is the real part. Dashed line is the imaginary part. . . . . . . . . . . . . . . . 


\section{List of Tables}

$2.1 X M M-N e w t o n$ X-ray Point Sources with NED Identifications . . . . . 63

2.2 Chandra X-ray Point Sources . . . . . . . . . . . 63

3.1 Masses and Radii ${ }^{\mathrm{a}}$ of Some Representative NFW Cluster Models in the Standard $\Lambda$ CDM Cosmology at $z=0$. . . . . . . . . . . . . . . 132

4.1 Effects of Non-Equipartition on the SZ-Mass Relation . . . . . . . 166

4.2 Characteristic Numbers of Clusters . . . . . . . . . . . . . . 166

4.3 Best-Fit Cosmological Parameters and Biases for Different $Y$ Versus Mass Calibrations . . . . . . . . . . . . . . . 167

5.1 Parameters for the IXO XMS Core and Full Arrays . . . . . . . . . . 204

5.2 Count Rates . . . . . . . . . . . . . . . . . . . . 204

5.3 Minimum and Maximum Redshifts . . . . . . . . . . . . . . . 204

A.1 Visibility Fitting . . . . . . . . . . . . . . . . . . 232 


\section{Chapter 1}

\section{General Introduction}

\subsection{Clusters of Galaxies}

Clusters of galaxies are the largest virialized systems in the universe (Sarazin 1986; Borgani et al. 2002; Voit 2005; Kaastra et al. 2008; Böhringer \& Werner 2010). They typically have total masses of the order of $10^{14} M_{\odot}$, with some extreme cases up to a few times $10^{15} M_{\odot}$ (e.g., Zhang et al. 2006; Schmidt \& Allen 2007; Umetsu et al. 2009). The typical radius is a few Mpc. They were first discovered in optical observations as regions with higher number densities of galaxies compared to the average value over the entire sky. The number of galaxies within a cluster of galaxies can be a few hundred up to thousands. An important study of galaxy clusters was done by Abell (1958) who has complied an extensive catalog of rich clusters, and the catalog is still widely used.

Although clusters of galaxies are first discovered optically, the major form of matter is dark matter which makes up of $\gtrsim 80 \%$ of the total mass (Vikhlinin et al. 2006; Bonamente et al. 2008). In fact, the earliest evidence for the existence of dark matter came from clusters of galaxies by the fact that clusters of galaxies are gravitationally 
bound (Zwicky 1937); they would not have been bound if there had been no dark matter. The X-ray, weak lensing, and galaxy distributions in the merging Bullet Cluster 1E0657-56 have provided some of the most direct evidence for the existence of dark matter in the universe (Clowe et al. 2004) and also have provided constraints to the nature (e.g., self-interaction cross section) of the dark matter (Markevitch et al. 2004; Randall et al. 2008).

The largest proportion of baryonic matter in clusters of galaxies is the diffuse Xray emitting hot plasma distributed over the whole cluster. This intracluster medium (ICM) has an electron number density ranging from $n_{e} \sim 10^{-2} \mathrm{~cm}^{-3}$ near the central regions of the densest clusters (cooling core clusters; Fabian 1994; McNamara \& Nulsen et al. 2007) to $\sim 10^{-5} \mathrm{~cm}^{-3}$ in the outermost regions of typical clusters (Tozzi et al. 2000; Roncarelli et al. 2006; Wong \& Sarazin 2009). Typical electron temperatures are $T_{e} \sim 10^{7}-10^{8} \mathrm{~K}$. The hot gas contributes about $15 \%$ of the total mass (LaRoque et al. 2006; Vikhlinin et al. 2006; Gonzalez et al. 2007; Sun et al. 2009; Dai et al. 2010). Galaxies and diffuse intracluster stars unbound from galaxies only contribute about 3-4\% to the total mass (Murante et al. 2004; Gonzalez et al. 2005).

Because the ICM is the dominant form of visible matter in clusters of galaxies, studying the ICM is essential to understanding the largest virialized systems in the universe. Galaxy clusters are also ideal systems to study plasma physics under extreme conditions that cannot be achieved in laboratories.

The most important observable from the ICM is X-ray emission primarily due to thermal bremsstrahlung of the very hot optically thin plasma. Most of our understanding about the physical state of the ICM is from measuring the X-ray surface brightnesses and spectra of the hot plasma (Sarazin 1986; Böhringer \& Werner 2010). 
Currently active X-ray observatories include Chandra, XMM-Newton, Suzaku, Swift, and RXTE. Chandra has very high spatial resolution $\left(\sim 1^{\prime \prime}\right)$, and can study the ICM structures down to a spatial scale of $\lesssim 0.1 \mathrm{kpc}$ for nearby clusters (e.g., the Virgo cluster; Böhringer et al. 2001; Young et al. 2002). X-ray spectra can be used to determine the density and electron temperature. On a larger spatial scale, the Suzaku X-ray observatory has sufficient surface brightness sensitivity to measure ICM properties out to radii of $r \sim 1-2$ Mpc (George et al. 2009; Reiprich et al. 2009; Basu et al. 2010; Hoshino et al. 2010). The future International X-ray Observatory (IXO) will have more than an order of magnitude improvement in both effective area and spectral resolution compared to any current mission. Therefore, X-ray observations will continue to be an important means to understand these gigantic systems for the foreseeable future.

Another important ICM observable is the Sunyaev-Zel'dovich (SZ) effect, which is a distortion in the Cosmic Microwave Background (CMB) spectrum due to electron scattering within a galaxy cluster (Sunyaev \& Zeldovich 1972; Birkinshaw 1999). Recently, SZ profiles have been measured for a number of clusters (e.g., Plagge et al. 2010). High angular resolution $\left(\sim 10^{\prime \prime}\right)$ SZ images have also been made by the MUSTANG bolometer on the Green Bank Telescope (Mason et al. 2010). Unlike the X-ray surface brightness which scales with redshift as $(1+z)^{-4}$, the SZ effect is independent of redshift. Thus, the SZ effect has the potential to probe ICM physics in high redshift clusters more easily than with X-ray observations, provided that the sensitivity of SZ observations is high enough and the contaminations from radio sources can be removed or well understood. 


\subsection{Importance to Cosmology}

The very high temperatures of the ICM are due to shock heating when clusters are formed during gravitational collapses. The formation of clusters and other large structures in the universe at high redshifts occurs when positive density fluctuations reach the critical density of the universe and collapse. These structures will continue to grow by the mergers of other groups and subclusters, and by accreting materials from the surrounding region (Gunn \& Gott 1972; Bertschinger 1985). During the process, the density of the collapsed cluster increases, and an accretion shock propagates outward from the cluster center and heats the gas. Cosmological simulations generally agree with the idea that the high temperature is caused by shock heating during the formation, but with a complication that clusters do not evolve in isolation (Navarro et al. 1995; Wechsler et al. 2002; Zhao et al. 2003a). These simulations show that the mass accretion history in general consists of two somewhat distinct phases. The first one is an early fast accretion phase which is dominated by major mergers of clusters, and the second one is a slow accretion phase which is dominated by smooth accretion of background materials and many minor mergers.

It is natural to tie the study of clusters of galaxies to cosmology due to the fact that galaxy clusters are the largest virialized structures in the universe (Voit 2005). Clusters of galaxies are important components of large-scale structure in the universe. They are often found at the intersections of the larger scale filamentary structures (Ryu et al. 2003; Springel et al. 2005; Ocvirk et al. 2008). Because the formation and growth of galaxy clusters depend on the primordial fluctuations and the expansion of cosmological background materials, the evolution of galaxy cluster abundance, or the mass function, is sensitive to cosmological parameters (Press \& Schechter 1974; Lacey \& Cole 1993; Jenkins et al. 2001). Recently, X-ray surveys 
have made significant progress in studying cosmology using galaxy clusters by using different techniques (e.g., the $f_{\text {gas }}$ technique: Allen et al. 2008; the mass function technique: Mantz et al. 2008; Vikhlinin et al. 2009). For example, using the mass function technique, an X-ray survey with a sample size as small as 85 X-ray clusters has already constrained some of the cosmological parameters down to a few percent level in statistical uncertainties (Vikhlinin et al. 2009). Ongoing and future SZ surveys will detect thousands of clusters, and this will significantly improve the statistics (Carlstrom et al. 2002; Bartlett et al. 2008). Given that systematic uncertainties will dominate over the statistical uncertainties in the future, it is important to control the systemic uncertainties due to galaxy cluster physics down to the percent level in order to use clusters for precision cosmological studies.

\subsection{Beyond Equilibrium Physics}

In general, clusters of galaxies can be crudely classified into merging clusters and relaxed clusters. Merging clusters have recently $(<1$ Gyr) had major merging events and their morphology is often complex. Merger shocks or cold fronts can often be identified with deep enough X-ray observations (e.g., Markevitch \& Vikhlinin 2007). Obviously, the merging systems are not in hydrostatic equilibrium. Relaxed clusters are systems which have not encountered major mergers recently. They are more regular and spherical symmetric in morphology (e.g., Vikhlinin 2006; Hudson et al. 2010). It is believed that hydrostatic equilibrium has nearly been reached in relaxed clusters. Because of the regularity of the relaxed clusters, it is relatively simple to study cluster properties in these systems compared to merging clusters. The systematic uncertainties in relaxed clusters are also smaller compared to merging clusters, and hence, studying relaxed clusters are particularly important for precision cosmology. 
Even for the most regular relaxed cluster, the assumption of hydrostatic equilibrium will break down in some regions. For example, it is now clear that many of the central regions of relaxed cool core clusters are disturbed by jets from Active Galactic Nuclei (AGNs; see Section 1.3.1 and Chapter 2). With the high spatial resolution X-ray observatories such as Chandra and XMM-Newton, it is possible to study these non-equilibrium dynamical processes in cluster cool cores (Fabian 1994; McNamara \& Nulsen et al. 2007).

The ICM is also usually assumed to be in collisional equilibrium so that fluid approximation can be used to describe the properties of the plasma. However, in the outer regions of clusters, the collisional timescale between electrons and ions can be so long that collisional equilibrium cannot be achieved (see, e.g., Fox \& Loeb 1997; Ettori \& Fabian 1998; Loeb 2007; Wong \& Sarazin 2009, and also Section 1.3.2, Chapters 3, 4, and 5). With improvements in the sensitivities of X-ray and SZ to measurements, signatures of these non-equilibrium processes should be observable. A careful study of the non-equilibrium physics in clusters of galaxies is important. If one wants to understand cluster physics at a percentage level and study cosmology using clusters to a comparable precision, studying the non-equilibrium effects may be unavoidable.

In this thesis, we study non-equilibrium processes in clusters of galaxies on these two different size scales. On the small size scale $(r \lesssim 50 \mathrm{kpc}$ to $r \sim 500 \mathrm{kpc})$, we study observationally a cool core cluster with AGN feedback. This research area is particularly active and the theory of the cool core clusters has experienced dramatic changes since the launch of Chandra and XMM-Newton (McNamara \& Nulsen et al. 2007). We observed the central region of a cool core cluster Abell 2626 which shows evidence in non-equilibrium processes such as AGN feedback and merging with an infalling subcluster. On the large size scale $(r \gtrsim 2 \mathrm{Mpc})$ on which much of the the- 
sis is focused, we study theoretically the non-equilibrium processes in the outermost accretion shock regions of galaxy clusters. These non-equilibrium processes are due to the low density and hence the long collisional timescale near the shock regions of galaxy clusters. Studying the outer regions of clusters is observationally challenging due to the very low surface brightness. Even with Suzaku, which is the most sensitive instrument we have, current observations can only measure X-ray emission out to around $r \sim 2 \mathrm{Mpc}$ where the non-equilibrium effects may not be very significant. However, there has already been some evidence from Suzaku observations that the electron pressure in cluster outer regions may be lower than that predicted by numerical simulations assuming collisional equilibrium (George et al. 2009; Basu et al. 2010; Hoshino et al. 2010). In the near future, combining Suzaku (for its high surface brightness sensitivity) and either Chandra or $X M M$ (for their high spatial resolution to remove point source contamination) may allow these outer cluster regions to be observed (Bautz et al. 2010). In the future, the IXO should be able to probe the ICM out to the shock radius $(r \sim 3-4 \mathrm{Mpc})$. In particular, IXO will have more than an order of magnitude increase in sensitivity compared to current missions, which will make the study of non-equilibrium processes in cluster outer regions much easier.

\subsubsection{Feedback from Central AGN: the Case of Abell 2626}

Very often, the ICM is be assumed to be adiabatic because the average cooling time

for a typical cluster, $t_{\text {cool }} \approx 58 n_{e,-3}^{-1} T_{e, 7.5}^{1 / 2}$ Gyr, where $T_{e, 7.5} \equiv T_{e} / 10^{7.5} \mathrm{~K}$, and $n_{e,-3} \equiv$ $n_{e} / 10^{-3} \mathrm{~cm}^{-3}$ (Sarazin 1986), is much longer than the age of the universe of 13.7 Gyr. However, in many clusters, the central densities can be so high that the cooling time can be less than the age of the cluster, and these are called cooling core clusters. In the classical cooling flow model, the rapidly cooling gas in this region leads to the 
loss of central pressure (Fabian 1994). If there is no external heating, the cooled gas would flow into the center in a cooling flow (Fabian 1994; McNamara \& Nulsen et al. 2007). The classical cooling flow model predicts that significant amounts of cool gas should be found at the centers of these clusters (Fabian 1994). Indeed, this is the case. However, both the Chandra and XMM-Newton found that the amounts of gas cooling to low temperatures were at least a factor of ten less than expected if the gas cooled due to its own radiation (Peterson et al. 2001, 2003). This suggests that the central gas has been heated by some external source instead of continuing to cool down. In addition, both observatories found that there are X-ray cavities near the center of these clusters (Fabian et al. 2000; Blanton et al. 2001). Very often, these clusters host central dominant galaxies that are strong radio sources (Burns 1990). Many of the X-ray cavities found are spatially coincident with the radio "bubbles" blown by the central radio sources. It is now widely believed that the radio plasma ejected by the central AGN is displacing the X-ray emitting ICM. The central AGN may be activated by accreting a small amount of cooler materials (e.g., the cooling ICM). The ejected radio jets may be heating up the surrounding ICM through this feedback process (McNamara \& Nulsen et al. 2007). The detailed microphysics of how the radio jets heat the ICM is still not well-understood (see, e.g., Fabian et al. 2006; Vernaleo \& Reynolds 2006; Sternberg \& Soker 2008). Clearly, with the feedback process from the central AGN, the central regions of a cooling core cluster can be dynamically complex. Part of the thesis is a study of the non-equilibrium processes in the cooling core cluster Abell 2626 using Chandra and XMM-Newton observations. This cluster shows evidence of X-ray emitting ICM interacting with radio jets from a central AGN. 


\subsubsection{Non-Equilibrium Processes in the Outer Regions of Clusters}

To a first order approximation, the ICM can be assumed to be in collisional equilibrium over most of the cluster except for the outermost regions. Most X-ray observations of most clusters have been limited to a radius of less than $\lesssim R_{500}{ }^{1}$ even with Chandra and XMM-Newton observatories. The electron number densities within this radius are of the order of $10^{-4} \mathrm{~cm}^{-3}$, and the Coulomb collisional timescale between electrons and proton is $t_{e p} \approx 1.1 T_{e, 7.5}^{3 / 2} n_{e,-4}^{-1}$ Gyr, where $n_{e,-4} \equiv n_{e} / 10^{-4} \mathrm{~cm}^{-3}$ (Spitzer 1962; Sarazin 1986). The Coulomb collisional timescale within $R_{500}$ is shorter than the age of clusters (a few Gyr), and hence, local thermodynamic equilibrium can be reached in the inner regions. However, in the outer regions $\left(\gtrsim R_{200}\right.$ up to the shock radius) of galaxy clusters where the electron density can be lower than $\sim 10^{-5} \mathrm{~cm}^{-3}$, the Coulomb collisional timescale can be longer than the typical ages of the clusters. Non-equilibrium effects may occur; electrons and ions may not be in equipartition (Fox \& Loeb 1997; Ettori \& Fabian 1998; Chieze et al. 1998; Takizawa 1999; Rudd \& Nagai 2009; Wong \& Sarazin 2009), and the ionization state of the gas may depart from collisional ionization equilibrium (CIE) (Yoshida et al. 2005; Cen \& Fang 2006; Yoshikawa \& Sasaki 2006; Akahori \& Yoshikawa 2008, 2010). If these non-equilibrium processes are not taken into account, the measured properties of the outer parts of clusters will be biased.

In this thesis, we study the non-equipartition effect and non-equilibrium ionization (NEI) effect in the outer regions of relaxed clusters. We use one-dimensional hydrodynamic simulations to study a sample of 31 clusters (groups) with masses between $10^{13}-10^{16} M_{\odot}$ under the $\Lambda$ CDM cosmology. Our one-dimensional simulations repro-

\footnotetext{
${ }^{1} R_{\Delta}$ is the radius within which the mean total mass density of the cluster is $\Delta$ times the critical density of the universe.
} 
duce the properties of the relaxed cluster outer regions simulated by three-dimensional simulations (Navarro et al. 1995; Ryu \& Kang 1997). We focus on a few representative clusters to study non-equilibrium effects on the X-ray and SZ properties of clusters. We also study the detectability of non-equilibrium effects with upcoming $I X O$ and Atacama Large Millimeter Array (ALMA).

\subsection{Thesis Outline}

The thesis is organized as follows. In Chapter 2, we present XMM-Newton and Chandra observations of Abell 2626 which shows evidence of two precessing jets interacting with the cooling core. This Chapter was published in the Astrophysical Journal (Wong et al. 2008). Chapter 3 describes the set up of the hydrodynamic models we used to study non-equilibrium processes in cluster outer regions, the physics of non-equilibrium processes which induce non-equipartition electrons and ions, and the non-equipartition effects on X-ray and SZ observables. This Chapter was published in the Astrophysical Journal (Wong \& Sarazin 2009). The impact of the non-equipartition effect on cosmological parameters estimation from $\mathrm{SZ}$ surveys is presented in Chapter 4. This Chapter was also published in the Astrophysical Journal (Wong et al. 2010). We present the non-equilibrium ionization calculations and the X-ray signatures in Chapter 5. This Chapter will be submitted to the Astrophysical Journal. We summarize our work and present the future outlook for the study of the non-equilibrium processes in galaxy clusters in Chapter 6. A brief note on the detectability of non-equipartition effect at cluster accretion shocks with ALMA is presented in Appendix A at the end of this thesis. 


\section{Chapter 2}

\section{XMM-Newton and Chandra}

\section{Observations of Abell 2626:}

\section{Interacting Radio Jets and Cooling}

\section{Core with Jet Precession?}

[Adapted from Wong, K.-W., Sarazin, C. L., Blanton, E. L., \& Reiprich, T. H. 2008, ApJ, 682, 155]

\section{$2.1 \quad$ Introduction}

The central regions of clusters of galaxies are among the most interesting and physically active areas in the Universe. In many clusters of galaxies, there is a central peak in X-ray surface brightness, with a central cooling time which is less than the age of the cluster (e.g., Chen et al. 2007). In the classical cooling flow picture, the rapid cooling of gas in this region leads to the loss of central pressure (Fabian 1994). If 
there is no additional heating, the cooled gas would flow into the center in a cooling flow. The gas should continue to cool to very low temperatures. However, recent high resolution X-ray spectra from $X M M$-Newton and Chandra show that there is not enough cooler X-ray gas to be consistent with a classical cooling flow (Peterson et al. 2001, 2003; Peterson \& Fabian 2006).

The lack of sufficient cooler materials probably means that there is an additional heating source within the central cooling core region. Very often, the clusters host central dominant galaxies which are also strong radio sources. In some of the clusters, there is strong evidence that the central radio sources are interacting with the surrounding X-ray emitting plasma. The radio sources blow "bubbles" in the X-ray emitting gas, displacing the gas and compressing it into shells around the radio lobes. At the same time, the radio sources are confined by the X-ray emitting gas (Fabian et al. 2000; Blanton et al. 2001). In some clusters, "ghost bubbles" at larger radii are seen which are weak in radio emission except at low frequencies (McNamara et al. 2001; Clarke et al. 2005). They are believed to be previous eruptions of the radio sources, which are episodic. In some cases, buoyantly rising bubbles may entrain cooler X-ray gas from the centers of the cooling cores (Churazov et al. 2001; Fujita et al. 2002, 2004). Some radio sources previously classified as cluster merger radio relics may actually be displaced radio bubbles from the central radio sources (Fujita et al. 2002, 2004). In some recent studies, there is even evidence of radio jets restarted at a different position angle from a previous outburst, which may be due to a precessing supermassive blackhole (Gitti et al. 2006). With high resolution 3D simulations, Vernaleo \& Reynolds (2006) have shown that multiple bursts from the AGN along the same direction fail to produce a long-term balance of heating and cooling. The reason is that a low-density channel is evacuated by a previous jet, 
while the subsequent jets can pass through the channel freely without heating the cooling core region. It has been suggested that jet precession is a possible solution to this problem. Alternatively, turbulence, rotation, or bulk motions in the intracluster medium (ICM) might also move or disrupt the channel made by the radio source, and keep the energy from just going down the same channel (e.g., Heinz et al. 2006).

Abell 2626 ( $z=0.0573$; Abell et al. 1989) hosts a moderate cooling flow of $\sim 50 M_{\odot} \mathrm{yr}^{-1}$ based on Einstein data (White et al. 1997). A double nuclei cD galaxy is sitting at the center of the cluster. From high resolution VLA images, Gitti et al. (2004) recognized that there is an unresolved radio core coincident with the central cD galaxy. There is a small jet-like feature extended towards the southwest direction from the unresolved radio core. In addition, there are two unusual amorphous, symmetric radio "bars" running parallel at opposite sides of the central $\mathrm{cD}$ galaxy. These compact features are distinct from and embedded in diffuse extended radio emission (a mini-halo). Gitti et al. (2004) believed that the radio bars may represent an earlier evolutionary stage of jets injected by the central source. Thus, Abell 2626 is a good candidate for studying the interaction between the X-ray emitting intracluster medium and the radio emitting plasma (Rizza et al. 2000; Gitti et al. 2004; Marković et al. 2004). Previous ROSATX-ray and VLA radio observations suggested that there is an X-ray excess which is spatially correlated with the radio source, but failed to find strong X-ray deficits (holes) in Abell 2626 (Rizza et al. 2000). Rizza et al. (2000) carried out numerical simulations, and showed that even a weak jet (internal Mach number $=5$ ) should produce a significant hole along the line-of-sight to the radio jet. They suggested that the lack of X-ray holes may be due to mixing of thermal and radio plasma in the region, although their numerical simulations did not distinguish between these two components. They also ran simulations in which the jet turned 
off, which resembled the observations of weak X-ray holes. Another possibility they noted is the imprecise model they used to subtract out from the symmetric X-ray emission to obtain a residual image. They searched for X-ray and radio correlations from the residual image but failed to find strong X-ray deficits (holes) displaced by the radio plasma. With the excellent spatial resolution provided by Chandra and deeper observations provided by both Chandra and XMM-Newton, we are able to study the complicated central region of Abell 2626 in more detail.

While many radio sources are associated with AGNs in cluster galaxies, there exist other types of extended radio emission associated with the diffuse intracluster medium without clear galaxy hosts. These extended radio sources can be divided into clusterwide halos, relics, and mini-halos (Feretti \& Giovannini 1996, 2008). Abell 2626 contains a candidate mini-halo (Gitti et al. 2004). Cluster-wide halos and relics are large in scale $(\sim 1 \mathrm{Mpc})$, with halos located in the centers of clusters, while the relics are generally located at the periphery. Very often, these two types of large scale diffuse radio emission are found in clusters without cooling cores. This indicates a possible origin in cluster mergers, which are believed to disrupt cooling cores and to re-accelerate radio-emitting particles (Brunetti 2004; Blasi et al. 2007). However, some cooling core clusters with dominant radio galaxies host mini-halos at the cluster center with extended radio emission up to $0.5 \mathrm{Mpc}$. The morphology of the mini-halos is different from that of the radio bubbles at the centers of many cool core clusters, which show a strong $\mathrm{X}$-ray/radio interaction in which the radio lobes displace $\mathrm{X}$ ray gas. This hints at a different transport mechanism (not jets) to explain the extended property of the mini-halos. The typical time scales for radiative losses by relativistic electrons $\left(\sim 10^{7}-10^{8} \mathrm{yr}\right)$ in cooling cores with strong magnetic fields are much shorter than the diffusion times $\left(\sim 10^{9}\right.$ yr $)$ for the electrons to be transported 
from the center of the cooling core out to a fraction of a Mpc. Hence, the diffuse radio emission from mini-halos suggests that an in-situ re-acceleration mechanism is needed for the electrons. The origin of the mini-halos has remained unclear, but it has been suggested that the interaction of the central radio galaxy and the intracluster medium (re-)accelerates particles to relativistic energies. Recently, Gitti et al. (2004) modeled Abell 2626 with an electron re-acceleration model driven by MHD turbulence amplified by the compression of the magnetic field in the cooling core region. They have shown that the model agrees with various observational constraints such as the observed radio brightness profile, the integrated synchrotron spectrum, and the radial steepening of the radio spectrum. They conclude that only a tiny fraction $(\sim 0.7 \%)$ of the maximum power that could be extracted from the cooling core (based on the standard cooling flow model) is needed for re-acceleration. Since the re-acceleration model for the origin of the mini-halo assumes a physical connection between thermal and relativistic plasma, it is essential to perform new X-ray observations to derive accurately the properties of the ICM (e.g., cooling radius, mass accretion rate, as well as morphology of the X-ray emission). On another hand, Pfrommer \& Enßlin (2004) suggested that the re-acceleration is of hadronic origin which should be best tested by $\gamma$-ray observations.

In this chapter, we present a detailed study of Abell 2626 from XMM-Newton and Chandra observations focused on the X-ray and radio interaction. The chapter is organized as follows. In Section 2.2, we describe observations and data reduction. The X-ray images are analyzed in Section 2.3, with an emphasis on the X-ray morphology of the overall region, the central region, and the S0 galaxy IC 5337 in Section 2.3.1. In particular, two extended X-ray emission regions possibly associated with subclusters are discussed in Section 2.3.1. The point sources detected in the X- 
ray data are discussed in Section 2.3.2. The X-ray surface brightness profile is studied in Section 2.3.3. Various substructures identified on residual maps are presented in Section 2.3.4. Spectral analysis is presented in Section 2.4. In particular, cluster profiles, mass deposition rate, cooling and thermal conduction time scales, hardness ratio maps, and possible AGNs associated with the $\mathrm{cD}$ galaxy IC 5338 and the S0 galaxy IC 5337 are discussed in this section. Throughout the data analysis sections (Sections $2.3 \& 2.4$ ), some discussions related to the interpretation of the results are presented to help readers understand the data. We then discuss the kinematics of the S0 galaxy and the cooling flow interaction with the central radio source in Sections 2.5 \& 2.6 in detail, respectively. We summarize our work in Section 2.7. We assume $H_{0}=71 \mathrm{~km} \mathrm{~s}^{-1} \mathrm{Mpc}^{-1}, \Omega_{M}=0.27$ and $\Omega_{\Lambda}=0.73$ throughout the chapter. At a redshift $z=0.0573$, the luminosity distance of Abell 2626 is $252.8 \mathrm{Mpc}$, and $1^{\prime \prime}=1.10 \mathrm{kpc}$. The virial radius is approximately $1.53 \mathrm{Mpc}$ (Markevitch et al. 1998) for the average cluster temperature of $T_{X}=3.0 \mathrm{keV}$ (White et al. 1997).

\subsection{Observations and Data Reduction}

\subsubsection{XMM-Newton Data}

Abell 2626 was observed (Obs. ID 0148310101) on 2002 December 28 by XMM-Newton for $\sim 38$ and $\sim 42 \mathrm{ks}$ with the EPIC PN and MOS cameras, respectively. Extended Full Frame mode (PN) and Full Frame mode (MOS) were used with thin optical filters. XMM-Newton data reduction was done with SAS version 5.4.1. All the data were reprocessed using the tasks epchain and emchain. We included only events with FLAG $=0$ in our analysis. We chose only events with PATTERN $=0-4(0-12)$ for PN (MOS) data. 
Background flares were rejected by performing a $2 \sigma$ clipping of the $100 \mathrm{~s}$ binned light curves in the 12-14 keV and 10-12 keV band for the PN and MOS cameras, respectively, which yielded the out-of-time corrected exposure times of 35,563 s, 40,300 s and 40,600 s of cleaned data for the PN, MOS1 and MOS2 cameras, respectively. Blank-sky background files collected by $\mathrm{D}$. Lumb ${ }^{1}$ were used during the analysis. The observed background levels, estimated from the total count rate in the whole field of view in the hard band (10-12 keV and 12-14 keV for MOS and PN, respectively), are 1.07, 0.99 and 1.26 times those in the blank-sky backgrounds for MOS1, MOS2 and PN, respectively. We have renormalized the blank-sky backgrounds accordingly. The uncertainty of the PN background is larger than that for the MOS detectors. The effect of the larger PN background uncertainty and some other background uncertainties on the spectral analysis are addressed in Section 2.4.1. The image and hardness ratio map analyses were done using the MOS data only (Sections $2.3 \& 2.4 .6$ ), and we checked that the results of the analysis would not be affected by a change in the background normalization of $\pm 10 \%$. The vignetting effect for the image and hardness ratio map analysis has been corrected using the exposure maps.

\subsubsection{Chandra Data}

A Chandra observation (Obs. ID 3192) of $\sim 25$ ks was made on 2003 January 22 in Very Faint mode. The center of the cluster was positioned $3^{\prime}$ from the edge of the S3 chip (midway between the node boundaries). The focal plane temperature was -120 C.

The Chandra data reduction was done with CIAO version 3.1, using calibration products from CALDB 2.28. The latest gain file (CALDB GAIN 2.28) and geome-

\footnotetext{
${ }^{1} \mathrm{ftp}: / /$ xmm.vilspa.esa.es/pub/ccf/constituents/extras/background/
} 
try file (CALDB 2.9) at the time of reduction were used. The reduction was done in Very Faint mode to remove more particle background. Background flares were rejected by performing the standard clipping using the $l c_{-}$clean routine written by M. Markevitch ${ }^{2}$. This yielded 22,959 s of cleaned data. Since the whole field-of-view is dominated by the cluster X-ray emission, internal background cannot be used. The blank-sky background files collected by M. Markevitch were used during the analysis. The difference between the observed and the blank-sky high energy (9-12 keV) background count rates was less than $1 \%$, and this small difference was removed by renormalizing the background. The influence of the background on the spectral fitted measurements (temperature profile) has been checked by varying the background normalizations by $\pm 10 \%$, and the results are not significantly changed. To check if there might be a significant difference between the Galactic background in the source and blank-sky observations, another thermal component with a fixed low temperature of $0.2 \mathrm{keV}$ has been added (Markevitch et al. 2003; Lumb et al. 2002). Normalization of the additional component has been allowed to be negative for the case of more Galactic emission in the blank-sky observations compared to the cluster observation. All the results are basically unchanged. Fixing the temperature of the additional component to be the outermost temperature that can be determined from the Chan$d r a$ field of view also gives essentially the same results. We conclude that the Chandra results are insensitive to uncertainties in the background.

\footnotetext{
${ }^{2}$ http://cxc.harvard.edu/contrib/maxim/acisbg/
} 


\section{$2.3 \quad$ X-Ray Image Analysis}

\subsubsection{X-ray Morphology}

\section{Global morphology}

The background subtracted, exposure-corrected, adaptively smoothed mosaic of the XMM-Newton EPIC MOS1 and MOS2 images is shown in Figure 2.1. The PN image was not included because of its different spectral response and because the chip gaps and bad columns produced cosmetic artifacts in the smoothed image. The image was smoothed to a signal-to-noise ratio of 3 per smoothing beam.

The global X-ray image of Abell 2626 appears to be roughly azimuthally symmetric. The brightest region at the center corresponds to the position of the $\mathrm{cD}$ galaxy IC 5338. There is a source associated with the cluster S0 galaxy IC 5337 which is 1.3 west of the center of the cluster. Excess X-ray emission is seen to the southwest of the cluster, extending from $\sim 1^{\prime}$ out to $\sim 7^{\prime}$ (see Section 2.3.4). Mohr et al. (1996) identified a subcluster to the southwest of this region based on optical observations of the galaxy population. The subcluster identified is centered roughly at the southwest edge of the XMM-Newton field of view, but the distribution of the galaxies covers a region where the excess X-ray emission is seen. Mohr et al. (1996) show that the subcluster is bound to and falling into the main cluster. Our XMM-Newton image shows, for the first time, extended X-ray emission possibly associated with the subcluster in the southwest direction. The extended X-ray emission can be viewed on a background subtracted, exposure corrected, adaptively smoothed image. The location of the extended emission possibly associated with the subcluster is circled at the southwest corner in Figure 2.1. The extended emission at the southwest corner is not obvious, but the slight deviation from spherical symmetry in that region can 
be noted. The shape of the extended emission region is difficult to characterize due to the low signal-to-noise ratio near the edge of the cluster. By inspecting various images, we chose a circular region centered on the highest excess X-ray emission, with a radius chosen to be small enough so that the region did not overlap with the nearly circular cluster emission of Abell 2626. Our choice for this region is justified by the hardness ratio map below (Figure 2.14) which shows that the extended region chosen appears to be harder than its surrounding. Compared to the X-ray emission at the same radius from the main cluster center (the $\mathrm{cD}$ galaxy IC 5338) with all point sources and other extended emission excluded, the excess emission is significant at the $4.6 \sigma$ level. Our residual images (Section 2.3.4 below) also indicate the possible signatures of a merger.

Another extended X-ray source can be seen about $7^{\prime}$ northeast of the center of Abell 2626 (Figure 2.1). The criteria used to define the northeast region are the same as the southwest one. This feature is significant at the $11.6 \sigma$ level. It should be noted that there is a chip gap at about 5.5 from the center which makes the extended X-ray source appear to be more distinct than it actually is. No cluster or group structure has been identified previously in this region. However, Abell 2626 is known to be associated with the Perseus-Pegasus supercluster, a filament of clusters of galaxies extending for as much as 300 Mpc (Batuski \& Burns 1985a,b; Zucca et al. 1993; Einasto et al. 2001). The southwest to northeast extension to the structure in our X-ray image around Abell 2626 is elongated along the Perseus-Pegasus filament. This may indicate that the cluster is preferentially accreting subclusters and groups from this supercluster. 


\section{Central Region}

The background subtracted, exposure corrected, adaptively smoothed Chandra image of the central $3.5 \times 2 ! 4$ is shown in Figure 2.2 (left panel). The image was smoothed to a signal-to-noise ratio of 3 . The brightest region of the X-ray emission in the cluster is centered on the southwest nucleus of the central cD galaxy. This source is possibly extended in both soft $(0.3-2 \mathrm{keV})$ and full band $(0.3-10 \mathrm{keV})$ images when detected by wavedetect in CIAO, but nothing was found by wavedetect in the hard band (2-10 $\mathrm{keV})$. However, examination of the raw soft band $(0.3-2 \mathrm{keV})$ image shows two peaks superposed on the two optical nuclei of the central $\mathrm{cD}$ galaxy (lower left panel of Figure 2.3). There is also a hard point source seen in the raw hard band $(2-10 \mathrm{keV})$ image (lower right panel of Figure 2.3). The position of this hard point source agrees with the southwest $\mathrm{cD}$ nucleus. The hard point source is separated from the peak of the radio core by less than $1^{\prime \prime}$. This source can also be seen in the hardness ratio map (Figure 2.16 below). The hard point source and its likely association with the AGN at the center of the southwest $\mathrm{cD}$ nucleus and radio core are discussed further in Section 2.4.7. This source is located along an arc of X-ray emission extending from the northeast to the west to the south of the cluster center.

Previous ROSATX-ray and VLA radio observations suggest that there is enhanced X-ray emission spatially coincident with the radio source (Rizza et al. 2000). In Figure 2.2 (left panel), the contours from the VLA $1.5 \mathrm{GHz}$ B-array radio image are shown in green (Gitti et al. 2004). The position of the radio core agrees with the brightest region of X-ray emission. In particular, the radio core is centered at the southwest $\mathrm{cD}$ nucleus rather than the northeast one. There is an arc of X-ray emission which may correspond to the curved radio jet feature to the south of the core.

However, the most unusual features of the radio image are the two elongated radio 
bars to the north and south of the radio core. We find there is no obvious correlation between the two radio bars and the Chandra X-ray image in Figure 2.2 (left panel). No obvious X-ray deficit (holes) is found on the Chandra X-ray image, which is consistent with the finding of Rizza et al. (2000). We have generated residual maps to further investigate our finding, and the results will be presented in Section 2.3.4. Our interpretation is that the radio bars are thin tubes (see Section 2.6 below).

One interesting feature is that there is an X-ray excess "tongue" which extends from the central $\mathrm{cD}$ galaxy to the southern radio bar, and there is a similar but weaker tongue in the northern direction as well (upper right panel of Figure 2.3). The tongues are best seen in the residual map in Figure 2.8 of Section 2.3.4.

Figure 2.2 (left panel) also shows radio contours (white) from the VLA $1.5 \mathrm{GHz}$ C-array (Gitti et al. 2004) overlaid on the Chandra image showing the radio mini-halo in Abell 2626. The mini-halo is roughly confined within $60^{\prime \prime}$ with a diamond shape. The distance from the center to the NE or SW corners of the diamond is about 50", while to the NW or SE corners it is about 70". A discussion on the mini-halo will be presented in Section 2.6.

\section{The S0 Radio Galaxy IC 5337}

About 1'.3 west of the central cD galaxy, there is an X-ray source associated with the S0 galaxy IC 5337 (left panel of Figure 2.2). This source is clearly extended in the soft band image, but a hard band image shows that there is a point source coincident with the nucleus of IC 5337. The possibility that the S0 nucleus is an AGN will be discussed below (Section 2.4.7). To the south of IC 5337, there is another X-ray point source which is not associated with any known galaxy. As indicated in Figure 2.2, there is a nearby radio source which may be associated with this X-ray source. 
The right panel of Figure 2.2 shows the bow-shock-like shape of the extended Xray emission associated with the $\mathrm{S} 0$ galaxy IC 5337. The image is created by removing and replacing the southern point source by the average surrounding X-ray intensity, and then adaptively smoothing to a signal-to-noise ratio of 4 .

The S0 galaxy IC 5337 also is a radio source (Figure 2.2). Radio contours (Gitti et al. 2004) are overlaid in Figure 2.2 (right panel). There is a component centered on the S0 galaxy, and a component located at a position of about $190^{\circ}$ (measured from north to east) of the S0 galaxy on the $1.5 \mathrm{GHz}$ B-array map. The $1.5 \mathrm{GHz}$ C-array map shows three radio tails in the south, southwest and west directions. The kinematics of the S0 galaxy will be discussed in Section 2.5.

\subsubsection{Source Detection}

We used the XMMSAS task eboxdetect to detect a total of 90 distinct point sources on the $X M M$ images; the detections were done separately on each of the three detectors, and then the source lists were combined. The sources were confirmed by inspection, and some low-level detections at the edge of the FOV or bad pixel gaps were removed. Here we discuss only the sources with possible optical counterparts within $20^{\prime \prime}$. In fact all optical counterparts identified are within $11^{\prime \prime}$. We adopt the NED source with an accurate position which is closest to the X-ray position, The possible associations of X-ray point sources with AGNs in the central cD galaxy IC 5338 and the S0 galaxy IC 5337 were noted above (Sections 2.3.1 \& 2.3.1), and will be discussed in more detail below (Section 2.4.7). Nine X-ray sources are included in the list (Table 2.1). Figure 2.4 is an optical image from the Digital Sky Survey with the 9 X-ray sources marked. Two of these are the central cD galaxy IC 5338 and the S0 galaxy IC 5337 discussed above. Interestingly, three of the X-ray sources correspond to galaxies 
which are located in the region of extended X-ray emission about $7^{\prime}$ northeast of the center of Abell 2626 (Figure 2.1). However, one of these possible optical IDs (2MASX $\mathrm{J} 23365722+2114032$ ) has a redshift of 0.038 , and thus is not directly associated with Abell 2626.

On the Chandra image, 14 point sources were detected by wavedetect in CIAO using the default settings. Of these, only the central cD IC 5338 galaxy and the S0 galaxy IC 5337 have possible identifications in NED with positions that agree with the X-ray to within $10^{\prime \prime}$. Table 2.2 lists the positions of the 14 Chandra X-ray point sources. Figure 2.5 is an optical image from the Digital Sky Survey with the locations of the 14 X-ray sources marked.

Unless specified, all the sources detected are excluded in the analysis of extended emissions in the subsequent sections.

\subsubsection{Surface Brightness Profile}

The azimuthally-averaged X-ray surface brightness profile was extracted separately for the Chandra and XMM-Newton data. Except for the two extended X-ray clumps to the NE and SW and the structure in the very center, Abell 2626 appears to be a nearly relaxed cluster. The profiles were fitted using a double beta model profile of the form

$$
S(r)=\sum_{i=1,2} S_{i}(0)\left[1+\left(\frac{r}{r_{c i}}\right)^{2}\right]^{-3 \beta_{i}+1 / 2},
$$

which has been shown to provide a good fit to relaxed clusters with cooling cores (Xue \& Wu 2000).

The background subtracted and exposure corrected surface brightness profile from the XMM-Newton MOS1 and MOS2 data is shown in Figure 2.6. The PN data were not included since the spectral response is different which complicates the deprojection 
of the electron density profile below (Section 2.4.3). Also, the fitted surface brightness model was used to create residual images (Section 2.3.4 below), and including the PN image would produce cosmetic artifacts due to the larger chip gaps and greater number of bad columns in the PN camera. All of the X-ray point sources except the central cD galaxy were excluded from the data. The extended X-ray emission around the S0 galaxy was also excluded, as it produced a feature in the cluster surface brightness profile. We also excluded the extended X-ray emission regions to the NE and SW of the cluster which were described in Section 2.3.1, and the criteria of defining the regions were also explained in that section. The annuli were chosen such that all the widths are larger than the FWHM of the PSF at that radius. With this choice, all the bins contain at least 1000 counts after background subtraction for the XMM-Newton MOS1 and MOS2 data. With these very good statistics, the error bars on the surface brightness are too small to be easily seen in the top panel of Figure 2.6; instead, we present the residuals in the lower panel. The parameters of the best-fit double beta model are $S_{1}(0)=0.40_{-0.02}^{+0.02}$ and $S_{2}(0)=0.10_{-0.01}^{+0.01}$ counts s $^{-1} \operatorname{arcmin}^{-2}, r_{1}=34.8_{-5.6}^{+7.1}$ and $r_{2}=128.5_{-7.2}^{+8.1}$ arcsec, and $\beta_{1}=0.91_{-0.18}^{+0.26}$ and $\beta_{2}=0.73_{-0.01}^{+0.02}$. Visually, the double beta model fit seems adequate. However, with the very small errors on the measured surface brightness, the fit is actually not very good, with a reduced $\chi^{2}$ of 2.3 . In addition to the excess at the center, associated with the central $\mathrm{cD}$ galaxy and radio source, within $200^{\prime \prime}$ there are several other regions of excess or deficient emission compared to the model. These features are reproduced (albeit with poorer statistics) in the Chandra surface brightness data, and in the data from the XMM-Newton PN detector. These residuals may indicate that the central region is disturbed by effects of the central radio source beyond the current extent of the source, or that the cluster is not completely relaxed due to a merger. 


\subsubsection{Residual Images}

To better understand the origin of the residuals in the surface brightness fit and the dynamics of the intracluster gas, we created residual maps for both XMM-Newton and Chandra images (Figures 2.7 and 2.8). Simulated azimuthally-symmetric X-ray images were produced which exactly followed the double beta model fits to the surface brightness. These simulated images were subtracted from the adaptively smoothed images. Figure 2.7 shows the XMM-Newton and Chandra residual images with the same field of view. Both residual maps show similar structures; the XMM-Newton image has great contrast due to the better statistics, while the Chandra image shows more detailed structures. Substructures can be identified with many of the residuals seen in the maps. A large region of excess emission can be seen about $110^{\prime \prime}$ southwest of the cD galaxy, which corresponds to the peak of the surface brightness residuals around that radius. Other regions of excess and deficit within $100^{\prime \prime}$ can also be identified from the residual map of the Chandra image (right panel of Figure 2.7). In the surface brightness residuals, the central excess corresponds to the central bright point in Figure 2.7 (right panel). The trough around $15^{\prime \prime}$ corresponds to the dark ring around the central excess in the residual map which can be seen more clearly on Figure 2.8. The peak at around $30^{\prime \prime}$ is probably due to the two bright regions due north and south in the residual map (labeled as two green circles). The trough around $50^{\prime \prime}$ corresponds to the dark region indicated by a white polygon in the residual map. The peak around $110^{\prime \prime}$ corresponds to the far southwest excess labeled with a green polygon in the residual map.

Most of the structures in the inner region occur on a scale similar to that of the radio sources in the center. In order to study the radio/X-ray interaction in more detail, Figure 2.8 shows radio contours from the VLA $1.5 \mathrm{GHz}$ C-array (white 
contours) and the $1.5 \mathrm{GHz}$ B-array radio images (green contours, Gitti et al. 2004), overlaid on the central part of the Chandra residual map. In general, the diamondshaped outline of the extended radio emission (the radio "mini-halo") corresponds to a region of reduced X-ray emission, and there are excess X-ray regions NNE and SSW of the edge of the mini-halo where the radio surface brightness is dropping rapidly. The mini-halo is roughly confined within $60^{\prime \prime}$. The distance from the center to the NE or SW corners of the diamond is about 50"; while to the NW or SE corners is about $70^{\prime \prime}$. Note that in the interpretation of Gitti et al. (2004), the two radio bars are distinct from the diffuse radio emission, and in fact after their subtraction the morphology of the mini-halo becomes roughly circular (see Figure 6 of Gitti et al. 2004). However, in the residual image in Gitti et al. (2004), we can still see some excess radio emission in the NW and SE corners. As noted previously, there is a significant excess associated with the radio core and the southern radio jet. The southern radio bar is a region of X-ray excess, but the northern bar shows an excess to the east and a deficit to the west in X-rays. The interpretation of the interaction between thermal and radio plasma will be discussed in Section 2.6 in detail.

The one major X-ray residual in the inner cluster which is not on the scale of the central radio emission is the extended excess to the SW. It is possible that this has been produced by the action of the central radio source. However, the SW excess is between the $\mathrm{cD}$ galaxy and the subcluster southwest of the main cluster center. It is possible that this excess is the result of this merger or a previous merger from this direction. There is no obvious sharp surface brightness discontinuity associated with this excess, which means that it either is not a shock or cold front, or that the merger is not occurring primarily in the plane of the sky. We note that Mohr et al. (1996) show that the line-of-sight velocity difference between the main cluster and 
the subcluster is $\sim 2600 \mathrm{~km} \mathrm{~s}^{-1}$, which is greater than the typical sound speed within clusters. If the excess is the result of a merger, this suggests that the merger is mainly along the line-of-sight.

\subsection{Spectral Analysis}

\subsubsection{Temperature Profile}

The azimuthally averaged, projected temperature profile for Abell 2626 was extracted using both XMM-Newton and Chandra data (upper panel of Figure 2.9). The spectra were fitted using an absorbed thermal model (WABS*MEKAL) with XSPEC Version 12.3.1. For the $X M M-N e w t o n$ data, all the spectra of the three cameras were fitted simultaneously. The energy bands used are $0.6-10.0$ and $0.4-7.0 \mathrm{keV}$ for $X M M$ Newton and Chandra data, respectively. Using 0.6-7.0 keV and 0.5-10.0 keV bands for XMM-Newton and Chandra data gives essentially the same results. The spectra were grouped to have a minimum of 25 counts per bin. We fixed the absorption to be $4.2 \times 10^{20} \mathrm{~cm}^{-2}$ (Dickey \& Lockman 1990) and the redshift to be 0.0573 (Abell et al. 1989). Freeing these values gives essentially the same results. Except the outermost data point where the reduced $\chi^{2}$ value is $\sim 1.6$, all the other reduced $\chi^{2}$ values are $\lesssim 1$, which indicates that the fits are good. To ensure that the spectra are not affected by energy-dependent PSF effects, the annuli of the XMM-Newton data were chosen such that all of the widths are at least twice the PSF half energy width of the PN camera.

The general trend is that the projected temperature increases from a central value of $\sim 2.5 \mathrm{keV}$ within $9^{\prime \prime}(\sim 10 \mathrm{kpc})$ to a maximum of $\sim 3.5 \mathrm{keV}$ at $100^{\prime \prime}-200^{\prime \prime}(110-$ $220 \mathrm{kpc})$. Such a radial increase is normally seen in a cluster cooling core. The 
projected temperature then decreases to $\sim 1.4 \mathrm{keV}$ at $\sim 540^{\prime \prime}(\sim 600 \mathrm{kpc})$. Interestingly, there is a sharp drop in the projected temperature from $\sim 2.9 \mathrm{keV}$ at $210^{\prime \prime}$ $(230 \mathrm{kpc})$ to $\sim 2.0 \mathrm{keV}$ at $270^{\prime \prime}(296 \mathrm{kpc})$ in the XMM-Newton data. Outside of this steep drop, the projected temperature gradient flattens. The radius of the drop corresponds to the distance from the $\mathrm{cD}$ galaxy to the outer edge of the large excess emission in the southwest direction. If the large excess emission indicates a merger signature, the temperature jump might indicate that gas is heated within that region. Unfortunately, the jump cannot be confirmed with Chandra as the Chandra data does not extend far enough in all directions to reach the distance of the jump seen in the azimuthally averaged temperature profile. It only extends far enough towards the northwest direction, where there are not enough counts to constrain the temperature.

In general, the $X M M-N e w t o n$ data give lower values for the projected temperature profile. There are at least three possible explanations for this difference. First, it might be due to the larger PSF of XMM-Newton. The softer photons from the brighter, cooler inner regions may be scattered to outer regions, hence making the outer regions appear cooler. This effect may be seen from the second bin outward, and should be more serious in regions where the gradient of the surface brightness is greatest (i.e., near the center; Markevitch 2002). This might explain the discrepancy in the second and third bins of our XMM-Newton data. The second possibility is that there might have been a steady and mild enhancement in the hard X-ray background during the Chandra observation which was not removed by the standard cleaning procedure; this could make the Chandra emission appear hotter, particularly in outer, low surface brightness regions. Such an effect has also been noted by Markevitch (2002). We have tried to assess the importance of such a background enhancement by including a component for the hard background, modeled as a power law with 
an exponential cutoff (Markevitch 2002; Markevitch et al. 2003) in the fits of the Chandra spectra. However, this component did not significantly improve the fits, and the best-fit amplitude of the component was negligible. A third possibility is that the continuous accumulation of the contaminant layer on the Chandra ACIS optical blocking filter was underestimated.

To study the true temperature of the cluster, we have also deprojected the temperature profile by using the XSPEC PROJCT model. For each annulus, the model sums up the appropriate fractions of emissions contributed from the outer annuli. The spectra of all the annuli were fitted simultaneously to get the deprojected temperature profile. The middle panel of Figure 2.9 shows the azimuthally averaged, deprojected temperature profile for Abell 2626 using both XMM-Newton and Chandra data. For the XMM-Newton data, all the spectra from the three cameras were fitted simultaneously. The XMM-Newton and Chandra data are consistent within the $90 \%$ confidence uncertainties, but the uncertainties of the Chandra data are much larger. Since the $X M M-N e w t o n$ data extends further out and has smaller error bars, we describe results obtained from the $X M M-N e w t o n$ in the following. We also adopt the XMM-Newton spectral fits (lower panel of Figure 2.9) for the deprojection and the mass profile analysis below (Sections 2.4.3, 2.4.4, \& 2.4.5).

The deprojected central temperature is slightly lower than the projected one because the latter is contaminated by the hotter emission along the line of sight. The difference is small since the hottest region has a temperature only $1 \mathrm{keV}$ higher than the central one. A more noticeable difference is that the temperature jump at around $240^{\prime \prime}$ is more obvious in the deprojected profile. It drops from a temperature of $\sim 3.5$ $\mathrm{keV}$ to $\sim 2.2 \mathrm{keV}$. The peak of the deprojected temperature profile is located just within the temperature jump $\left(\sim 180-240^{\prime \prime}\right)$. 


\section{Effects of XMM Background Uncertainties}

Since the cluster emission covers the whole field of view and the correct background cannot be estimated easily, the so called "double background subtraction" method, common in XMM-Newton data analysis (Arnaud et al. 2002), cannot be applied. The natural background subtraction method is to perform a renormalized blank-sky background subtraction without the second step of extracting a blank-field-subtracted spectrum in an emission free region in the "double background subtraction" method. We use this "single background subtraction" method. Note that this method has also been applied to the study of a cluster with a similar redshift to Abell 2626 (Arnaud et al. 2001). As mentioned in Arnaud et al. (2001), our treatment does properly account for the cosmic-ray (CR) induced background. However, the contributions from variations in the local soft Galactic background (which depends on the position on the sky) and the extragalactic X-ray background component (which depends on the absorbing hydrogen column density along the line of sight and cosmic variance) were not included. In our case, the emission from Abell 2626 usually dominates the local soft Galactic background, and the CR induced background usually dominates the hard X-ray extragalactic background component. Using the ROSAT All Sky Survey (Snowden et al. 1997), we have compared the soft X-ray emission (0.47-1.21 keV) around Abell 2626, and the exposure-weighted emission in the regions used to extract the blank-sky background, and the difference is less than $4 \%$. The hydrogen column density towards Abell 2626 is about a factor of two higher than that for most of the blank-sky fields (Dickey \& Lockman 1990), but is not high enough to affect the cosmic X-ray background strongly. Also, as mentioned previously, the CR induced background usually dominates the hard X-ray extragalactic background component. Hence, the blank-sky backgrounds should represent the soft Galactic background and 
the hard X-ray background reasonably.

We were concerned that, while the background renormalizations for MOS1 and MOS2 are modest $(\lesssim 7 \%$ correction), the renormalization of the PN background is larger ( $\sim 26 \%$ correction). While the uncertainties in the background will affect most strongly the derived temperatures of the outer regions of the cluster, we emphasize here that most of our focus in this chapter is on the X-ray and radio interaction in the innermost region where the results are nearly independent of background assumed. We performed a number of tests to address the effects of the $X M M$ Newton background uncertainties on the deprojected temperature profiles. We did this by: 1) varying the blank-sky background normalizations by $\pm 10 \%$ ( $\pm 20 \%)$ for the MOS (PN); 2) using the blank-sky backgrounds without renormalization; 3) using the MOS1+MOS2 spectra only; 4) using the PN spectra only; 5) adding an extra $0.2 \mathrm{keV}$ MEKAL component (with normalization free and allowed to be negative in each annulus) as a model for any variation in the soft Galactic component. The test results are summarized in Figure 2.10. Our adopted deprojected temperature profile in this chapter is shown in black diamonds.

Within $r<180^{\prime \prime}$, all the deprojected temperatures are in very good agreement. All these tests show a significant jump in temperature near 240" and a flattening of temperature beyond this jump. As expected, increasing the background normalization (blue diamonds) gave lower deprojected temperatures for the outer regions $(r>$ $180^{\prime \prime}$ ), while decreasing the background normalization (green diamonds) gave higher temperature. Using the blank-sky backgrounds without renormalization (red crosses) also gave higher temperatures. The variation in the temperatures using different normalizations was at most $\sim 1 \mathrm{keV}$ in the outer region.

The deprojected temperature profiles obtained from the MOS1+MOS2+PN spec- 
tra (black diamonds), the MOS1+MOS2 spectra (magenta triangles), and the PN spectra alone (cyan triangles) all agree reasonably well. Thus, the PN data appear to give fairly reliable spectral information, even though the uncertainty in the PN background normalization may be larger.

Finally, varying the amount of soft Galactic background (orange crosses) gave deprojected temperatures in good agreement with those using the renormalized blank sky backgrounds (black diamonds) except for the outermost annulus. The large deviation of the outermost data point may indicate that there is less of soft Galactic emission in the direction of Abell 2626 than in the blank-sky fields because the fitted normalization of the extra soft Galactic background component in the model is negative. The soft Galactic component should be uniform on the scale of a cluster. However, a reduction in the soft Galactic background similar to that suggested by the last annulus was found to be completely inconsistent with the fits in the inner annuli. Hence, we concluded that the reduced soft component in the outermost annulus was not due to a reduction in the soft Galactic background towards the cluster. The reduced soft component in the outermost annulus may be the result of additional thermal structure in this region.

To summarize, all our results are independent of background used within $\sim 180^{\prime \prime}$. For the outer regions, the temperature profile is only slightly affected by the choice of the background, but the differences are mostly well within the error bars. All the features described in the chapter related to temperature measurements (e.g., the temperature jump, pressure, entropy profiles, etc.) are not affected. 


\subsubsection{Central X-ray Spectrum and Cooling Rate}

We determined the radiative cooling rate by fitting the X-ray spectrum of the central regions of the cluster from both XMM-Newton and Chandra data. We extracted the spectrum from a circular region with a radius of $65^{\prime \prime}$, which is roughly the cooling radius determined in Section 2.4.4 below. In XSPEC, we used the WABS*(MKCFLOW+MEKAL) model, which combines a cooling flow component (MKCFLOW) with an isothermal model (MEKAL) to account for the gas in the outer regions. The absorption was fixed at the Galactic column of $4.2 \times 10^{20} \mathrm{~cm}^{-2}$ as before. The upper temperature $\left(k T_{\text {high }}\right)$ and abundance of the MKCFLOW component was set to be the same as the MEKAL component, which is expected if the gas cooled from the ambient ICM. Initially, the lower temperature of the MKCFLOW component is set to be the lower limit for the model $\left(k T_{\text {low }}=0.08 \mathrm{keV}\right)$, as expected for the classical cooling flow model in which the gas cools radiatively to very low temperatures. With this assumption, the best-fit cooling mass deposition rate is $2 \pm 1(3 \pm 3) M_{\odot} \mathrm{yr}^{-1}$ for XMM-Newton (Chandra) data in the 0.6-10.0 (0.5-7.0) keV energy band with a $\chi^{2} / d o f=1.18(0.90)$. The fitted upper temperature and abundance were $k T_{\text {high }}=2.8 \pm 0.05(3.3 \pm 0.2) \mathrm{keV}$ and $0.52 \pm 0.03(0.9 \pm 0.1)$ solar, respectively. Allowing $k T_{\text {low }}$ to vary increased the mass deposition rate to be $74 \pm 6 M_{\odot} \mathrm{yr}^{-1}$ for the $X M M-N e w t o n$ data, with $k T_{\text {low }}=1.5 \pm 0.1 \mathrm{keV}, k T_{\text {high }}=4.2_{-0.5}^{+0.2} \mathrm{keV}$, and an abundance of $0.45_{-0.02}^{+0.03}$ solar, with a $\chi^{2} / d o f=1.13$. The Chandra data gave very poor constraints on the mass deposition rate or lower temperature with a $\chi^{2} / d o f=0.87$ if $k T_{\text {low }}$ was allowed to vary. From the f-test, the probability that the fit to the model with $k T_{\text {low }}$ fixed is better than the fit with freeing $k T_{\text {low }}$ is only $2 \times 10^{-11}\left(9 \times 10^{-3}\right)$ for the XMM-Newton (Chandra) data. For comparison, the mass deposition rate determined from Einstein data by surface brightness deprojection was $\sim 53 M_{\odot} \mathrm{yr}^{-1}$ 
(White et al. 1997). As has been found in many other clusters (e.g., Peterson et al. 2003), the spectral data for Abell 2626 can be fit with significant amounts of gas cooling, but only by a factor of $\sim 3$ in temperature.

\subsubsection{Density, Pressure and Entropy Profiles}

Assuming the cluster is spherically symmetric, which appears to be roughly true for Abell 2626, the radial surface brightness profile (Figure 2.6) can be deprojected to give the X-ray emissivity $\epsilon(r)$ and electron density $n_{e}(r)$ as a function of radius (Appendix A in this chapter). The electron density profiles determined from $X M M$ Newton and Chandra data are shown in the upper panel of Figure 2.11. Except for more fluctuations in the Chandra data, the two results for radii greater than $\sim 7^{\prime \prime}$ are in good agreement. To avoid artifacts due to the assumption of zero emissivity outside the boundary during the deprojection (Takizawa et al. 2003), we have removed the 2 outermost points in each of the profiles plotted in Figure 2.11. In the Chandra data, we also note that the two innermost data points are higher than the $X M M$ Newton results by a factor of $\sim 1.5-2$. Such a deviation in the innermost data points can also be seen when comparing the surface brightness profile of Chandra data to $X M M-N e w t o n$ data (not shown in this chapter). The surface brightness profile, as well as other profiles, is centered at the location of the southwest nucleus of the $\mathrm{cD}$ galaxy. The central deviation between Chandra and XMM-Newton might be due to the X-ray contribution of a central AGN (Section 2.4.7) and the differences in the PSFs of XMM-Newton and Chandra. The smaller PSF of Chandra allows the central excess to be resolved more readily. We did not remove the central source during the analysis, since it is probably extended in the $0.3-10 \mathrm{keV}$ band (see Section 2.4.7). Also, including or excluding this region does not change any other conclusions in the 
chapter. The central excess extends to $4^{\prime \prime}-7^{\prime \prime}$ in radius, which is larger than the PSF of Chandra. This probably indicates that the excess is not due to an AGN alone. The northeast nucleus of the cD galaxy is located at about $4^{\prime \prime}$ away from the central AGN (Figure 2.3). From the residual map (Figure 2.8), we clearly see that there is an extended excess around the northeast nucleus. There is also excess emission northwest of the AGN, which can also be seen in the soft band raw image of Figure 2.3.

The XMM-Newton data show that the electron density decreases from $\sim 0.02 \mathrm{~cm}^{-3}$ at $\sim 7^{\prime \prime}$ to $\sim 0.0004 \mathrm{~cm}^{-3}$ at $\sim 400^{\prime \prime}$. The density profile is generally smooth, with a small jump around $60^{\prime \prime}$ seen in the Chandra data. However, the jump is not visible with the XMM-Newton data. This radius corresponds to the location where the logarithmic slope of the surface brightness profile changes, or equivalently, to where the surface brightness goes from being dominated by the smaller to the larger of the two individual $\beta$ models. We also noticed that the radio mini-halo is roughly confined within $\sim 60^{\prime \prime}$. Interestingly, this radius is also roughly coincident with the cooling radius (Section 2.4.4 below). Within the cooling radius and the region of the radio mini-halo, radiative cooling and/or the effects of the relativistic particles and magnetic fields in the radio plasma may affect the density profile of the X-ray gas.

The gas density and temperatures profiles can be combined to give the gas pressure $P=n k T$, where $n$ is the total number density in the gas (both ions and electrons). We assume a fully ionized plasma with half cosmic abundances of heavy elements which gives $n \approx 1.92 n_{e}$. We also derive the gas entropy parameter $S \equiv k T / n_{e}^{2 / 3}$ (Ponman et al. 2003). Since the spatial resolution of our density profile is much higher than the temperature profile, interpolation is used to determine the temperatures on the finer density grid by fitting a high order polynomial (lower panel of Figure 2.9). We assigned temperature errors of $5(20) \%$ at radii of smaller (larger) than $250^{\prime \prime}$. 
Using a constant temperature model gives similar results, except for more negative (unphysical) values for the total mass profile (Section 2.4.5 below). Thanks to the nearly constant temperature profile of Abell 2626, we believe that the interpolation of the temperature profile is reasonably accurate.

The pressure profile (middle panel of Figure 2.11) looks similar to the electron density profile, since the temperature is roughly constant. The feature at $60^{\prime \prime}$ becomes more obvious, as the slope of temperature is negative in this region. At around $240^{\prime \prime}$, there is a slope change in the pressure profile. This is associated to the temperature jump at that radius, which may be a merger signature.

The entropy profile is shown in the lower panel of Figure 2.11. There is a small bump in the entropy profile around $240^{\prime \prime}$ which is associated with the temperature jump and the change in slope of the pressure profile in the same region. The entropy may be boosted by a merger in that region. Excluding this entropy bump region, the logarithmic slope of the entropy profile in the outer regions $\left(\sim 100^{\prime \prime}-400^{\prime \prime}\right)$ is $0.58 \pm 0.06$, which is significantly smaller than the universal scaling relation slope of $\sim 1.1$ (e.g., Ponman et al. 2003). Note that there is a large scatter in the entropy profiles of individual clusters (e.g., Figure 1, Ponman et al. 2003), and hence the smaller logarithmic slope might not be unusual. There is a difference in the central entropy measured by XMM-Newton and Chandra. This central entropy deviation comes from the difference in central gas density measurements, which is probably due to the combination of a central excess in X-ray emission and differences in the PSFs of Chandra and XMM-Newton. However, the spatial resolutions of the temperature measurements are not sufficient to determine if the entropy differences are due to the temperature in the hot gas, or a central X-ray source. In the following, we chose to present results of the XMM-Newton data with which the spatial resolution 
in density profile measurement is poorer. The $X M M-N e w t o n$ data show that the entropy decreases from the outer value of $\sim 360 \mathrm{keV} \mathrm{cm}^{2}$, eventually flattening to a value of $\sim 30 \mathrm{keV} \mathrm{cm}^{2}$. If there is no feedback mechanism, a pure radiative cooling model will result in a power law entropy profile, with central entropy lower than $\sim 10 \mathrm{keV} \mathrm{cm}^{2}$ within $10 \mathrm{kpc}$ (Figure 1 of Voit \& Donahue 2005). The central entropy pedestal value of $\sim 30 \mathrm{keV} \mathrm{cm}^{2}$ implies that there has to be heating in the central region, which can probably be explained by a central AGN.

\subsubsection{Cooling and Thermal Conduction Time Scales}

Three important processes, heating, cooling and heat conduction, determine the thermal structure in the hot gas (e.g., Sarazin 1986). While the heating process in the central region is probably due to the AGN, the other processes are related to the hot gas structure itself. Here we compare two important time scales for the hot gas - the cooling and thermal conduction time scales. For the temperature gradients seen in typical cooling flow clusters, conduction is likely to be an important process if it is not suppressed by the magnetic field.

The cooling time was determined for each radius in the deprojected density profile using the values of the deprojected density and the deprojected temperature for both XMM-Newton and Chandra data. The rate of total emission from the gas was determined using the same MEKAL model used to fit the spectra. The integrated, isobaric cooling time $t_{\text {cool }}$ was determined. A brief outline of the cooling time estimation procedure is given in Appendix B in this chapter.

We define the conduction time scale to be (Sarazin 1986)

$$
t_{\mathrm{cond}} \equiv \frac{n_{e}(T /|\nabla T|)^{2} k}{\kappa}
$$


where $\kappa$ is the thermal conductivity for a hydrogen plasma (Spitzer 1962),

$$
\kappa=4.6 \times 10^{13}\left(\frac{T}{10^{8} \mathrm{~K}}\right)^{5 / 2}\left(\frac{\ln \Lambda}{40}\right)^{-1} \operatorname{ergs~s}^{-1} \mathrm{~cm}^{-1} \mathrm{~K}^{-1}
$$

and $\Lambda=37.8+\ln \left[\left(T / 10^{8} \mathrm{~K}\right)\left(n_{e} / 10^{-3} \mathrm{~cm}^{-3}\right)^{-1 / 2}\right]$ is the Coulomb logarithm.

Figure 2.12 shows the cooling and conduction time scales as a function of angular radius. The cooling time scale (plus symbols) decreases from $\sim 50$ Gyr at $\sim 540^{\prime \prime}$, down to $\sim 1$ Gyr at $\lesssim 15^{\prime \prime}$. Assuming the cluster has not changed much since $z \sim 1$, we define the cooling radius to be where $t_{\text {cool }}$ is equal to the time since $z=1$, which is 7.7 Gyr. The cooling radius, $r_{\text {cool }}$, is thus $\sim 60^{\prime \prime}-70^{\prime \prime}(\sim 66-77 \mathrm{kpc})$. Interestingly, $r_{\text {cool }}$ is of the same size as the mini-halo (see Section 2.3.4). It is also located near the radius where the logarithmic slope of the surface brightness profile changes, or equivalently, where the surface brightness profile goes from being dominated by the smaller to the larger of the two individual $\beta$ models (Section 2.3.3).

The conduction timescale depends on the temperature gradient, which is strongly affected by the uncertainties in the individual temperature points. The error estimation for the conduction timescale is complicated by the fact that the adjoining deprojected temperatures used to derive the conduction timescale are correlated. Hence, in calculating the uncertainties by error propagation, we included the full covariance matrix for the temperatures obtained from the deprojection using XSPEC. In the upper panel of Figure 2.12, the crosses show the values of the conduction time derived using the individual temperature values derived from annular XMM-Newton spectra (Section 2.4.1). The conduction time derived from the Chandra data (lower panel of Figure 2.12) are in general shorter than those from the $X M M-N e w t o n$ data. This may be due to the much larger scattering of the Chandra deprojected temperature profile. The error bars of the Chandra data are much larger than that of the XMM-Newton 
data. In order to reduce the effects of the uncertainties of the Chandra data, we also determine the conduction time scale in the inner region using the same interpolated temperature profile which was used to deproject the pressure/entropy profile (Section 2.4.3). The interpolated temperature profile was also used for the XMM-Newton data for comparison. These values are shown as dots in Figure 2.12; the upper panel gives the values for the XMM-Newton data, and the lower panel is for the Chandra data. The Chandra conduction time scales are longer than the XMM-Newton values in the innermost regions due to the higher densities derived there from the Chandra data.

Here we describe results obtained from XMM-Newton since the error bars are much smaller than those from Chandra. In the central regions of Abell 2626, the temperature gradient is very flat. The overall temperature variation is only about $0.4 \mathrm{keV}$ within the cooling radius of $\sim 65^{\prime \prime}$. Both the best-estimate and the lower bound on the conduction time are larger than the cooling time in the central regions of the cluster. The nearly isothermal temperature profile in Abell 2626 and resulting low conduction imply that thermal conduction is not important compared to cooling anywhere the cooling rate is high. Hence, in Abell 2626 the thermal structure within the cooling radius of $\sim 65^{\prime \prime}$ is probably determined by cooling and heating processes only. At a radius of $\sim 240^{\prime \prime}$, the conduction time scale drops to $\sim 0.3$ Gyr only, which is significantly shorter than the look-back time to a redshift $z \sim 1$. Even including the uncertainty in the conduction timescale, the upper limit on the time scale is still quite short. This indicates that either the thermal structure (perhaps associated with a merger) happened within $\sim 0.3 \mathrm{Gyr}$, or thermal conduction is suppressed at least by a factor of $\sim 20$. Except the feature at $\sim 240^{\prime \prime}$, outside of $\sim 65^{\prime \prime}$, we cannot determine whether thermal conduction is important or not due to the large uncertainties. 


\subsubsection{Mass Profiles}

Figure 2.13 shows the gas mass and total mass profiles of Abell 2626. The gas mass profile is given by

$$
M_{\text {gas }}(<r)=\int_{0}^{r} 4 \pi\left(r^{\prime}\right)^{2} d r^{\prime} \rho_{\text {gas }}\left(r^{\prime}\right)
$$

where $\rho_{\text {gas }}=\mu_{e} n_{e}$ is the gas mass density, and $\mu_{e}$ is the mass per electron determined from the gas abundances.

The total mass of the cluster can also be determined if we assume the hot gas is in hydrostatic equilibrium, and if the temperature of the hot gas is known. The total mass profile is given by (e.g., Sarazin 1986):

$$
M_{\mathrm{tot}}(<r)=-\frac{k T r}{\mu m_{p} G}\left(\frac{d \ln \rho_{\mathrm{gas}}}{d \ln r}+\frac{d \ln T}{d \ln r}\right) \text {, }
$$

where $\mu$ is the mean mass per particle, and $m_{p}$ is the proton mass. The temperature profile was interpolated from the XMM-Newton data (Figure 2.9, Section 2.4.3). Since the mass within any given radius is determined by local gas properties and their derivatives, the total mass can decrease or can even be negative. (This occurred at $r=57^{\prime \prime}$ for XMM-Newton and at a number of points for Chandra, and these points were omitted from the plot.) This can be due to measurement uncertainties in the data, which are compounded by the derivatives, or by the breakdown of simple hydrostatic equilibrium. The radio mini-halo is within $\sim 60^{\prime \prime}$, and may be disturbing the hot gas or contributing additional pressure support in this region (Sections 2.3.1 \& 2.3.4). Thus, the mass profile at small radii may not be represented accurately by hydrostatic equilibrium. At radii of $r \gtrsim 60^{\prime \prime}$, the general trend is for $M(<r)$ to increase with radius.

Compared to White et al. (1997) rescaled to our value of the Hubble constant, the 
gas masses we have obtained agree to within $2 \%$ and $7 \%$ at $321^{\prime \prime}$ and $489^{\prime \prime}$, respectively. The total mass agrees within $26 \%$ at $321^{\prime \prime}$. The difference in the total mass at this radius is large, but the values still agree within the uncertainties. The two values of the total mass do not agree at $489^{\prime \prime}$, probably due to the larger uncertainty in the temperature in the outermost annulus. The gas mass fraction $\left(M_{\text {gas }} / M_{\text {tot }}\right)$ increases from $\sim 5 \%$ at $100^{\prime \prime}$ to $\sim 10 \%$ at $500^{\prime \prime}$. The global trend is the same as is typical in relaxed clusters (David et al. 1995; Ettori \& Fabian 1999; Allen et al. 2002).

\subsubsection{Hardness Ratio Maps}

To understand the rough spectral properties of different regions, we have created hardness ratio maps from both XMM-Newton and Chandra data (Figures 2.14, 2.15, \& 2.16). For $X M M-N e w t o n$ data, the $\mathrm{PN}$ image was not included because of its different spectral response and because the chip gaps and bad columns produced cosmetic artifacts in the smoothed image. The hardness ratio maps were created by dividing the image in the hard band $(2-10 \mathrm{keV})$ by the image in the soft band $(0.3-2 \mathrm{keV})$. Each image was background-subtracted, exposure-corrected, and smoothed with the same scales as the $3 \sigma$ adaptively smoothed image of the $0.3-10 \mathrm{keV}$ band. We also tried smoothing all images with the same scales as the $5 \sigma$ adaptively smoothed image of the $0.3-10 \mathrm{keV}$ band, or with the same scales as the $3 \sigma$ adaptively smoothed image of the hard band (2-10 keV), and almost all the features look the same as the hardness ratio maps given in this chapter. While some features are not consistently seen in both the XMM-Newton and Chandra hardness ratio maps, indicating the possibility that they are statistic fluctuations in at least one of these maps, the common features which show up in both maps in the inner regions should be real (Figure 2.15). The colors from black to blue to red to yellow to white represent the degree of hardness 
from soft to hard. Though the hardness ratio maps from XMM-Newton and Chandra cannot be directly compared due to different spectral responses, the relative hardness is still useful to identify interesting regions.

\section{Global Structure}

Figure 2.14 is the hardness ratio map of the whole Abell 2626 from XMM-Newton data, overlaid with green contours of excess emission from the $X M M$-Newton residual map (left panel of Figure 2.7). Part of the apparent inhomogeneity in hardness is just due to noise. Also, near the blue and dark boundary of the hardness ratio map, the features start to be dominated by the hard background. Although difficult to see due to the rather flat temperature profile, the overall picture is a soft core at the center. At larger radii, the hardness increases outward to a radius indicated around the central cyan (light blue) circle, and then the hardness decreases outward. The central cyan circle is at a radius of $240^{\prime \prime}$ where the jump in temperature was identified (Section 2.4.1). From the hardness ratio map, it seems that the harder gas is confined within the cyan circle.

The white circles in Figure 2.14 are the two extended X-ray emission regions associated with the merging subclusters (Section 2.3.1). The discontinuities in the green contours near the white circles are actually artifacts of chip gaps. Both of the extended X-ray emission regions appear to be harder than their surroundings, and each of them contains a softer point-like source. The soft source in the northeast extended X-ray emission region is located at the peak of the extended emission, and is probably associated with the galaxy 2MASX J23365372+2113322 (Table 2.1), while the soft source in the southwest region is not associated with any identified galaxy. The two soft point-like sources in the two extended emission regions disappeared 
when the images were smoothed with the same scales as the $5 \sigma$ adaptively smoothed image of the $0.3-10 \mathrm{keV}$ band, or with the same scales as the $3 \sigma$ adaptively smoothed image of the hard band $(2-10 \mathrm{keV})$.

\section{Central Region}

The details of the central region of the hardness ratio maps are shown in Figure 2.15. Contours of excess emission are shown from the Chandra residual map (right panel of Figure 2.7). While the XMM-Newton and Chandra hardness ratio maps do not agree completely, probably due in part to noise and in part to the larger PSF of $X M M-N e w t o n$, we can still identify some common features.

One interesting feature is that the S0 galaxy IC 5337 shows a softer tail in the western direction. On the Chandra hardness ratio map (right panel of Figure 2.15), there is a narrow tail of soft emission starting from the southern corner of the IC 5337 tail and extending to the WSW. We also see a softer region with a similar scale in the XMM-Newton hardness ratio map (left panel of Figure 2.15).

The northern excess region $\sim 30^{\prime \prime}$ above the $\mathrm{cD}$ galaxy (contours) appears softer, with an extension towards the northeast direction which can also be seen in Figure 2.14. If the region is near hydrostatic equilibrium and the material is supported by ideal gas pressure, we would expect the brighter (and hence denser) regions to have softer spectra. This is roughly the case for the northern excess region. However,

for the southern excess at a similar radius where the tongue is located (Section 2.3.1), the brighter excess region does not correspond to a softer region, which suggests a breakdown of hydrostatic equilibrium. In particular, the X-ray excess tongue in the south direction of the $\mathrm{cD}$ galaxy (compared to Figure 2.8) appears to overlap both hard and soft regions extending in the same direction, making it hard to understand 
its origin.

Between the main cluster and the southwest subcluster, the large extended region of excess emission near the southwest corner appears to have a harder spectrum in the Chandra data (right panel of Figure 2.15), while it appears to be soft in the $X M M$-Newton data (left panel of Figure 2.15). From the hardness ratio map, it is not clear whether the extended excess is a shock heated region or a cold front.

In Figure 2.16, we can see clearly that there are two hard point sources located at the position of the cD galaxy IC 5338 and the S0 galaxy IC5337 (arrows). The interpretation of two AGNs will be discussed in Section 2.4.7. Radio contours (Gitti et al. 2004) are overlaid on the Chandra hardness ratio map to show in detail the complicated thermal and radio structures in the central region. The southern radio bar appears to be in a harder region, while the northern one appears softer. If the radio bars are indeed thin tubes along the plane of the sky, the X-ray emission along the line-of-sights may mainly be contributed by the cluster emission beyond the central region. In this case, the projected image or hardness ratio would not necessarily reflect the X-ray emissivities or hardnesses of the radio bars, and hence the lack of symmetry or a clear correspondence with features in the X-ray images or hardness maps might be understandable.

\subsubsection{Possible X-ray AGNs in IC 5338 and IC 5337}

We searched for evidence of a central point source associated with the $\mathrm{cD}$ galaxy IC 5338, which may indicate the existence of an X-ray AGN. When wavedetect was run on the $0.3-10 \mathrm{keV}$ and $0.3-2 \mathrm{keV}$ band Chandra images (Section 2.3.2), each detected one central source located at the southwest and northeast nucleus of the cD galaxy, respectively. Both were possibly extended. We also ran wavedetect on the 
hard band (2-10 keV) image, but it did not detect a central point source. However, when we inspected the hard band image, we noticed a possible hard point source. This source can be seen in the Chandra hardness ratio map (Figure 2.16). We extracted the HST image of the center of IC 5338 from the archive. The 300 s exposure was taken on 1999 June 26 using the HST WFPC2 camera with the F555W filter. Figure 2.3 shows clearly that this cD galaxy has two distinct nuclei, with the northeast nucleus being brighter optically. The two optical nuclei are located at the peaks of the soft X-ray image, with the radio core source in IC 5338 centered on the hard X-ray, southwest nucleus. The hard X-ray point source is only separated from the peak of the radio core by less than $1^{\prime \prime}$. Thus, we believe that this source is the AGN, and that the point-like hard X-ray source arises from this AGN.

To study the X-ray properties of the IC 5338 AGN, Chandra data were used to do photometry. Due to the limited number of photons, we cannot fit the spectrum of the AGN. Hence, we estimated the properties of the cD AGN by assuming that it has a power law spectrum with a photon index $\Gamma$. We extracted the total photon counts in a circular region with a 3 pixel radius in both hard $(2-10 \mathrm{keV})$ and soft $(0.3-2 \mathrm{keV})$ bands. In order to subtract the cluster emission as well as true background, we took background from a 3-pixel-wide annulus just outside the central region. The program $P I M M S^{3}$ was used to determine the photon index and the unabsorbed flux which gave the correct hardness ratio and photon counts. We assumed that absorbing column was given by the Galactic value $N_{H}=4.2 \times 10^{20} \mathrm{~cm}^{-2}$ (Dickey \& Lockman 1990). The best fit photon index is $\Gamma=2.2_{-0.4}^{+0.7}$. The unabsorbed flux in the $0.3-10.0 \mathrm{keV}$ band is $F_{X}=1.6_{-0.4}^{+0.7} \times 10^{-14} \mathrm{ergs} \mathrm{cm}^{-2} \mathrm{~s}^{-1}$, which gives an X-ray luminosity of $L_{X}=1.2_{-0.3}^{+0.5} \times 10^{41} \mathrm{ergs} \mathrm{s}^{-1}$ in the same energy band.

We also searched for evidence for a point source associated with an AGN in the

\footnotetext{
${ }^{3}$ http://heasarc.gsfc.nasa.gov/Tools/w3pimms.html
} 
center of the X-ray bright S0 galaxy IC 5337. In this case, wavedetect did detect a point source in the Chandra hard band $(2-10 \mathrm{keV})$ image at the center of IC 5337. This point-like, hard source is clearly seen in the hardness ratio map (Figure 2.16). The S0 galaxy is also a radio source, and the $\mathrm{X}$-ray point source is apparently associated with this central AGN. Again, the point source was too faint for spectral fitting. Using the same method described above, we determined the best-fit spectral index and flux assuming the Galactic hydrogen column density of $4.2 \times 10^{20} \mathrm{~cm}^{-2}$. The best fit photon index is $\Gamma=0.2_{-0.3}^{+0.6}$, the unabsorbed flux in the $0.3-10.0 \mathrm{keV}$ band is $F_{X}=$ $3.2_{-1.7}^{+1.9} \times 10^{-14} \mathrm{ergs} \mathrm{cm}^{-2} \mathrm{~s}^{-1}$, and the X-ray luminosity is $L_{X}=2.4_{-1.3}^{+1.5} \times 10^{41} \mathrm{ergs} \mathrm{s}^{-1}$. This spectrum index seems to be low (hard) for a typical AGN. One possibility is that there is excess internal absorption associated with the S0 galaxy AGN. To assess this, we fixed the photon index to $\Gamma=1.5$. In this case, the best-fit hydrogen column density is $N_{H}=1.3_{-0.8}^{+0.7} \times 10^{22} \mathrm{~cm}^{-2}$, the unabsorbed flux is $F_{X}=(3.0 \pm 1.5) \times 10^{-14}$ ergs $\mathrm{cm}^{-2} \mathrm{~s}^{-1}$, and the X-ray luminosity is $L_{X}=(2.3 \pm 1.1) \times 10^{41} \mathrm{ergs} \mathrm{s}^{-1}$. Somewhat counterintuitively, the extra absorption gives a lower unabsorbed flux because of the steeper spectral index assumed.

\subsection{Kinematics of the S0 Galaxy IC 5337}

The bow-shock-like shape suggests that the S0 galaxy is moving east directly towards the cluster center (right panel of Figure 2.2). The hardness ratio maps of the center of the cluster (Figures $2.15 \& 2.16$ ) show a region of soft (and presumably, cool) $\mathrm{X}$-ray emission extending to the west of the S0 galaxy. This geometry would be consistent with the S0 galaxy falling into the cluster from the west; the cool tail of gas would be either interstellar or intragroup gas associated with this galaxy which is being stripped by ram pressure from the motion through the ICM of Abell 2626 . 
The steepness of the intensity gradient in the cluster radio mini-halo on the western side compared to the eastern side (left panel of Figure 2.2) may also indicate that it is compressed by the S0 galaxy.

The radio structure complicates the interpretation of the motion of the galaxy, since there is a component centered on the S0 galaxy, and a component located at a position of about $190^{\circ}$ (measured from north to east) of the S0 galaxy on the 1.5 GHz B-array map (Figures 2.2, 2.8, \& 2.16). The $1.5 \mathrm{GHz}$ C-array map shows three radio tails in the south, southwest and west directions. If the south component is a Narrow-Angle-Tail (NAT) behind the S0 galaxy, then it is likely to be moving to the NNE (about $10^{\circ}$ measured from north to east) direction. On the other hand, the other two components (west and southwest tails) are more consistent with the bowshocked structure in the X-ray image which indicates that the S0 galaxy is moving to the east.

The interpretation of the kinematics of IC 5337 is confused by an ambiguity in the literature concerning its radial velocity. There are two optical determinations of the radial velocity which give values which are consistent with the velocity of other galaxies in the center of Abell 2626, including the cD galaxy IC 5338. Huchra et al. (1999) give a velocity of 16,485 $\pm 37 \mathrm{~km} \mathrm{~s}^{-1}$, and Falco et al. (1999) find 16,562 \pm 60 $\mathrm{km} \mathrm{s}^{-1}$. These values are consistent with the $21 \mathrm{~cm}$ line determination of $16,485 \pm 40$ $\mathrm{km} \mathrm{s}^{-1}$ by Giovanelli \& Haynes (1993). On the other hand, Mohr et al. (1996) list an optical velocity of $18,903 \pm 39 \mathrm{~km} \mathrm{~s}^{-1}$, and Karachentsev \& Kopylov (1981) list a velocity of $19,097 \mathrm{~km} \mathrm{~s}^{-1}$ with an uncertainty of the order of $100 \mathrm{~km} \mathrm{~s}^{-1}$. The difference between these velocities and the others is much greater than the quoted (or any likely) measurement errors. The velocities given by Mohr et al. (1996) and Karachentsev \& Kopylov (1981) would be inconsistent with IC 5337 being a part 
of the main central cluster Abell 2626. On the other hand, these higher velocities are consistent with IC 5337 being a member of the southern subcluster with a mean velocity of $19,164 \pm 138 \mathrm{~km} \mathrm{~s}^{-1}$ (Mohr et al. 1996). Most of the other members of this subcluster lie considerably to the south.

If IC 5337 is a member of the southern subcluster, its dynamics would probably follow somewhat the motion of the subcluster. We would expect IC 5337 would be infalling from SSW to NNE (towards the position angle of $30^{\circ}$ direction measured from north to east). This would be inconsistent with our interpretation of the bowshaped X-ray region to the east of IC 5337. Based on the X-ray image, we suggest that IC 5337 is falling directly into the main cluster from the west, and may not be associated with the southern subcluster. On the other hand, given that there are two velocity measurements consistent with the kinematics of the southwest subcluster (Mohr et al. 1996; Karachentsev \& Kopylov 1981), it seems unlikely that both are in error in the same way. One possible explanation is that there are two galaxies located along the line-of-sight toward IC 5337. Indeed, on the Chandra X-ray image, there are two point sources identified within the extended optical emission of IC 5337. One is located at the center of the S0 galaxy with two west and southwest NATs, while the other is located at its southern edge with a south NAT. The southern Xray source was removed during the analysis related to the S0 galaxy in this chapter. We have accessed the public available $\mathrm{IR}^{4}$, optical ${ }^{5}$ and $\mathrm{UV}^{6}$ surveys to search for a point source at the location of the southern X-ray point source. While there is no obvious emission in the IR $\mathrm{H}$ and $\mathrm{K}$ band images, there seems to be extended emission associated with the southern X-ray source in the IR J band, optical band and the UV band images. However, one cannot easily determine if this emission is a separate

\footnotetext{
${ }^{4}$ 2MASS: http://irsa.ipac.caltech.edu/Missions/2mass.html

${ }^{5}$ The STScI Digitized Sky Survey: http://stdatu.stsci.edu/cgi-bin/dss_form

${ }^{6}$ GALEX: http://www.galex.caltech.edu/
} 
galaxy or a feature in the disk of the S0 galaxy.

\subsection{Cooling Flow Interaction with the Central Ra- dio Source?}

The most unusual features of the radio image of Abell 2626 are the two elongated radio bars to the north and south of the radio core (Figures 2.2, 2.8, \& 2.16). Their symmetric positions suggests they are radio lobes, but their elongated shapes are unusual. By comparison to radio lobes associated with other cooling core dominant radio galaxies, one might expect these to be regions of reduced X-ray emission surrounded by bright rims ("radio bubbles", Fabian et al. 2000; Blanton et al. 2001). In radio bubbles, the radio plasma has apparently displaced the X-ray gas. One would expect the level of reduction in the X-ray surface brightness to depend on the extent of the bubbles along the line-of-sight. If they are highly oblate flattened regions with a large extent along the line-of-sight, the reduction in the X-ray surface brightness might be quite significant. If they are highly prolate tubes, then the reduction would be small. In fact, there is no obvious correlation between the two radio bars and the Chandra X-ray image, residual map or hardness ratio map in Figures 2.2, 2.8, \& 2.16, respectively. This may indicate that the radio bars are thin tubes, with a small extent along the line-of-sight, or that the radio plasma is mixed with the X-ray gas. In the numerical simulations by Robinson et al. (2004), if the magnetic field of the bubble is weak, the bubble is very unstable and can be destroyed easily, leaving no obvious

density contrast. The ratio of gas to magnetic pressure in the center of Abell 2626 is of the order of 100 (Rizza et al. 2000; Gitti et al. 2004), which is the same order as the weak magnetic field in the simulation by Robinson et al. (2004). However, it 
should be noted that the magnetic field in the radio bars could be much higher. One concern with this model is that it seems unlikely that such mixing would preserve the narrow radio bars which are observed.

One possible interpretation of the arc-like X-ray feature near the center of Abell 2626 (upper right panel of Figure 2.3) is that the central cD galaxy is moving to the west relative to the local intracluster gas. This could explain the bending of the X-ray emission near the center and the inner radio jets, as well as the elongation of the radio bars. However, in this case one might expect that the western edges of the two radio bars would indicate the positions where the radio jets have stopped. These positions are not mirror symmetric about the position of the radio core, as might be expected for jets perpendicular to the motion of the nucleus of the $\mathrm{cD}$ galaxy. Even if the jets are not perpendicular to the motion of the nucleus, one of the western edges of the two radio bars should have been behind the nucleus of the $\mathrm{cD}$ galaxy in this interpretation.

There are two X-ray excess "tongues" from the central cD galaxy to the two radio bars, with the southern "tongue" appears to be stronger (upper right panel of Figure 2.3, \& Figure 2.8). A similar tongue has been seen in Abell 133, where it was suggested that it might arise from a cluster merger, Kelvin-Helmholtz instabilities around the core, the buoyant uplift from a radio bubble, or a cooling wake (Fujita et al. 2004). Although subclusters may be merging with the outer regions of Abell 2626, there is no clear evidence for merger distortion in the central region. Even though the two cD nuclei may indicate a previous merger, the two nuclei are well inside the cD galaxy, which means that any associated merger may have happened a long time ago. Also, a merger might be expected to produce a tongue and radio lobe on only one side of the nucleus, as in Abell 133. For Abell 2626, the mean line- 
of-sight velocity of the galaxies is $16,533 \mathrm{~km} \mathrm{~s}^{-1}$, while that of the central cD galaxy is $16,562 \mathrm{~km} \mathrm{~s}^{-1}$ (Mohr et al. 1996), so the radial velocity of the $\mathrm{cD}$ galaxy relative to the cluster is low. Moreover, there is no obvious cold or shock front, which means that any motion in the plane of the sky is also slow. Therefore, there is no obvious velocity for the $\mathrm{cD}$ galaxy to induce Kelvin-Helmholtz instabilities. If the tongue is caused by a cooling wake, there would be only one. But we see two of them, though the northern one is much weaker than the southern one.

Eliminating the explanations by a cluster merger, Kelvin-Helmholtz instabilities and the cooling wake, the tongue seems to be best explained by the buoyant uplift from a radio bubble. In fact, a recent simulation indicates that when a bubble rises in the intracluster medium, large rolls extend out and converge under the bubble. This results in an upwelling and compression of material under the wake of the bubble (Robinson et al. 2004). The morphology of the southern tongue shown in Figure 2.8 looks very similar to the simulated result (Robinson et al. 2004) if we assume that the radio bar is the head of the plume. However, if the southern "tongue" is caused by uplifted cool gas, why is the northern X-ray tongue associated with the northern radio bar much weaker, given the two radio bars are similar in appearance? While there is no radio flux measurement to the radio bars in the literature, the stronger of the radio emission from the southern bar can be noted on the VLA $330 \mathrm{MHz}$ B+DnC-array radio map of Figure 4 in Gitti et al. (2004). This may explain the stronger uplift of the thermal plasma in the south.

Jet precession might also provide a natural explanation of the structure of the central region. The southwest cD nucleus is an AGN (see Section 2.4.7), and may be ejecting two jets towards the north and south. We assume the two jets are precessing about an axis which is nearly perpendicular to the line-of-sight along the north-south 
direction, so that the jets sweep out two conic surfaces to the north and south. If the two jets are stopped at approximately equal radii from the AGN (at a "working surface"), and if radio emission is produced by particles accelerated when the jets are stopped, then the radio bars might be produced as the jets precessed. If the jets are narrow, then the radio emission at the working surface may be narrow, explaining the shape of the radio bars and also the lack of anticorrelation between the two radio bars and the X-ray emission. The precession of the jets might be due to the gravitational effects of the second $\mathrm{cD}$ nucleus to the north, and this nucleus might have disrupted or weakened the northern X-ray tongue. Alternatively, the dominance of the southern X-ray tongue may simply be due to the southern jet being more powerful. There is also evidence of jet precession in other cooling core clusters (Gitti et al. 2006). Jet precession has also been suggested to be a requirement for the AGN feedback mechanism to solve the cooling flow problem (Vernaleo \& Reynolds 2006).

Abell 2626 is one of the clusters associated with a possible radio mini-halo, an extended diffuse radio source in the cooling core region of the intracluster medium (Gitti et al. 2004). In the cooling core region, the magnetic field strength is expected to be high due to compression. Consequently the radiative cooling time of the relativistic electrons is expected to be as short as $\sim 10^{7}-10^{8} \mathrm{yr}$, which is much shorter than the time needed to travel across the extended region (Gitti et al. 2004). In order to explain the existence of the radio mini-halo, an in-situ re-acceleration mechanism is needed. The steep radio spectrum of the mini-halo ( $\alpha \sim 2.4$, Gitti et al. 2004) may be due to confinement by the X-ray gas and radiative losses by the relativistic electrons.

While there is no very obvious correlation between the radio emission from the mini-halo and the X-ray emission from the X-ray images (Figure 2.2), there is some evidence of interaction between the radio mini-halo and the surrounding intracluster 
medium in the residual maps (Figure 2.8). In general, the diamond-shaped outline of the extended radio emission (the radio "mini-halo") corresponds to a region of reduced X-ray emission, and there are excess X-ray regions NNE and SSW of the edge of the mini-halo where the radio surface brightness is dropping rapidly. The mini-halo is roughly confined within $60^{\prime \prime}$. The distance from the center to the NE or SW corners of the diamond is about 50"; while to the NW or SE corners is about 70". As noted previously (Section 2.3.4), in the interpretation of Gitti et al. (2004) the two radio bars are distinct from the diffuse radio emission, and in fact after their subtraction the morphology of the mini-halo become roughly circular (see Figure 6 of Gitti et al. 2004), though we can still see some excess radio emission in the NW and SE corners. Interestingly, there is also a small density jump in the X-ray gas around 60" (Section 2.4.3 above). The size of the mini-halo is also coincident with the cooling radius (Section 2.4.4 above). This supports the re-acceleration model for the origin of the mini-halo (Gitti et al. 2004), though it could just be a coincidence since the definition of the cooling radius is somewhat arbitrary.

An excess in the residual map corresponds to a region where the gas density is higher than in the symmetric model (Figure 2.8). The shape of the mini-halo, if it is really elongated in shape (after proper subtraction of the radio bars), may be due to variations in the X-ray gas density. The radio source may be more strongly confined by the gas in the NE and SW directions. The points on the diamond-shaped radio mini-halo to the NW and SE may indicate regions of lower gas density where the radio plasma has been able to expand more. On the western side of the radio mini-halo, the intensity gradient is steeper than that of the eastern side. This may be due to gas compression by the S0 galaxy IC 5337 falling towards the cD galaxy IC 5338. If this is the case, then IC 5337 should be very close to the central cooling 
core, rather than being part of the southwest subcluster. This reinforces both our dynamical picture for the S0 galaxy and the interpretation of the mini-halo geometry by confinement. The X-ray deficient regions in the NW and SE directions can also be seen on $X M M-N e w t o n$ residual map on a larger scale (dark regions of the left panel of Figure 2.7). The excess of intracluster gas density to the NE and SW might be due to mergers along the axis of the local large scale structure, as discussed in Section 2.3.1, or it may just represent the elongation of the equilibrium distribution of the gas in this direction. The lower density regions in the NE and SW directions can also be regions inflated by previous radio bubbles. Although the symmetric radio bars may be due to the jet precession, it is also possible that the denser gas to the NE and SW might explain the shape of the two radio bars; the radio bars might have been compressed radially by a collision with this gas, and become extended to the NW and SE by the smaller ICM density gradients in those directions.

\subsection{Conclusions}

We have identified two extended X-ray emitting regions about $7^{\prime}$ northeast and southwest from the center of Abell 2626, with the latter one probably associated with a subcluster identified to be falling into the main cluster (Mohr et al. 1996; Mohr \& Wegner 1997). We argue that the infalling velocity of the southwest subcluster is mainly along the line-of-sight. Abell 2626 is known to be associated with the Perseus-Pegasus supercluster (Batuski \& Burns 1985a,b; Zucca et al. 1993; Einasto et al. 2001), and it is likely that the main cluster is accreting subclusters and groups from this large-scale structure (Sections 2.3.1, 2.3.4, \& 2.4.6). From the hardness ratio map, both of the extended X-ray emitting regions appear to be harder than their surroundings, and each of them contains a softer point-like source. The northeast point-like source may 
be associated with the galaxy 2MASX J23365372+2113322.

Based on the bow shape of the X-ray emission and the soft X-ray tail identified by the hardness ratio map, we argue that the S0 galaxy IC 5337 is falling towards the center of the main cluster from the west. The steeper intensity gradient in the cluster radio mini-halo on the western side compared to the eastern side may also indicate that the radio plasma is compressed by the S0 galaxy. This reinforces our interpretation that the S0 galaxy should be close to the cooling core region instead of located inside the southwest subcluster. On the other hand, if the S0 galaxy were associated with the subcluster in the southwest direction, this motion would be hard to understand. While there are two velocity measurements of IC 5337 consistent with the southwest subcluster and three measurements consistent with the main cluster, one possible explanation is that there may be two galaxies along the line-of-sight of IC 5337.

We have identified hard X-ray point sources due to AGNs associated with the $\mathrm{cD}$ galaxy IC 5338 and the S0 galaxy IC 5337, both of which are radio sources. The cD galaxy AGN is located on the southwest nucleus of the $\mathrm{cD}$ galaxy. The S0 galaxy AGN shows evidence for excess internal absorption.

We have used a double beta model to fit the radial surface brightness profile and generated residual images to understand the dynamics of the intracluster gas (Section 2.3.4). Most of the residual structures in the inner region occur on a scale similar to that of the radio sources in the center. One major residual in the inner cluster which is not on the scale of the radio emission is the extended excess to the southwest. It is located between the $\mathrm{cD}$ galaxies and the southwest subcluster, and may be the result of a merger.

The gas temperature in Abell 2626 increases from a central value of $\sim 2.5 \mathrm{keV}$ 
at $\sim 10 \mathrm{kpc}$ to a maximum of $\sim 3.5 \mathrm{keV}$ at around $230 \mathrm{kpc}$, and then decreases to $\sim 1.5 \mathrm{keV}$ at $\sim 600 \mathrm{kpc}$. At a radius of $\sim 260 \mathrm{kpc}$, there is a significant temperature jump seen in the fits to the XMM-Newton spectra. The jump may be associated with a previous or an ongoing merger. We used the X-ray spectra to determine the cooling rate within a projected radius of $72 \mathrm{kpc}$. The amount of gas which is cooling down to very low temperatures is less than a few $M_{\odot} \mathrm{yr}^{-1}$. A much higher mass deposition rate of $\sim 74 M_{\odot} \mathrm{yr}^{-1}$ is obtained from the $X M M$-Newton spectra if the hot gas only cools by about a factor of three.

From the surface brightness and the temperature profiles, we have derived the density, pressure, and entropy profiles of Abell 2626. At a radius of $\sim 70 \mathrm{kpc}$, there is a slope discontinuity in the density and pressure profiles. The outer entropy profile has a logarithmic slope of $\sim 0.58$, which is lower than that of the universal scaling relation of $\sim 1.1$. A heating source is probably required to maintain the central entropy of $\sim 30 \mathrm{keV} \mathrm{cm}{ }^{2}$. At a radius of $\sim 260 \mathrm{kpc}$, there is a small bump in the entropy profile which may be associated with a merger.

Due to the nearly isothermal structure of Abell 2626 within the cooling radius, conduction is not important compared to cooling in the inner region. The cooling radius is $\sim 70 \mathrm{kpc}$, where the logarithmic slope of the surface brightness profile changes. At a radius of $\sim 260 \mathrm{kpc}$, the conduction time scale is significantly shorter than the look-back time to a redshift of $z \sim 1$, which indicates that either the thermal structure (perhaps associated with a merger) happened recently or thermal conduction is highly suppressed. We have also derived the total mass profile under the assumption of hydrostatic equilibrium, and compared the gas mass fraction with other relaxed clusters. The global trend is typical for relaxed clusters.

We have investigated the cooling flow interaction with the central radio source. 
The two elongated radio bars to the north and south of the center of the cD galaxy and the lack of obvious correlation between the two radio bars and any structures in the Chandra X-ray image, residual image, or hardness ratio map may indicate that the radio bars are thin tubes parallel to the plane of the sky. Another possibility is that the radio plasma is mixed with the X-ray gas, rather than displacing it.

The central radio structure may be due to jet precession. The southwest cD nucleus is an AGN, and we suggest it has two precessing jets propagating towards the north and south, producing the two symmetric narrow radio bars. The precession might be caused by the gravitational influence of the second $\mathrm{cD}$ nucleus to the north. The two jets may have uplifted cool gas from the central region, producing the Xray excess "tongues" running from the center of the $\mathrm{cD}$ galaxy to the radio bars. The northern tongue appears to be weaker, and it may be disrupted by the northern nucleus of the $\mathrm{cD}$ galaxy. Alternatively, the strength of the southern X-ray tongue may simply be due to the southern jet being stronger. Jet precession may also be found in other cooling core clusters (Gitti et al. 2006). It may help to solve the AGN feedback problem found in high resolution simulations (Vernaleo \& Reynolds 2006). Alternatively, the distortions of the radio source might be due to ICM motion, particularly rotation, near the center of the cluster.

The radio mini-halo has a diamond shape, elongated in the northwest and southeast direction. The diamond-shaped radio mini-halo seems to be compressed from the northeast and southwest direction, which is probably consistent with the merging scenario for Abell 2626. With this compression geometry, the radio mini-halo might be leaking out in the northwest and southeast direction (Figure 2.8). The western side of the mini-halo may also be compressed by the infalling S0 galaxy IC 5337. Interestingly, the size of the mini-halo is roughly the same as the cooling radius, and is 
located at where the logarithmic slope of the surface brightness profile changes. The agreement between the size of the radio mini-halo and the cooling radius is consistent with the re-acceleration model for the origin of the mini-halo (Gitti et al. 2004), although it might just be a coincidence since the definition of the cooling radius is somewhat arbitrary. Independent of the origin of the radio mini-halo, the change in the X-ray surface brightness slope at the outer edge of the radio mini-halo may indicate that the radio plasma is affecting the thermal plasma. 


\section{Appendix A Density Deprojection}

We basically followed Kriss et al. (1983) to deproject the gas distributions from surface brightness profile. The procedure is briefly outlined here.

Assuming spherical symmetric gas distribution, the observed X-ray surface brightness (in ergs $\mathrm{cm}^{-2} \mathrm{sec}^{-1} \mathrm{~Hz}^{-1} \mathrm{sr}^{-1}$ ) at an angle $\theta$ (corresponding to a projected physical radius $b$ ) from the center of the cluster is:

$$
I_{\nu}^{\mathrm{obs}}(\theta)=\frac{1}{4 \pi(1+z)^{3}} \int_{b^{2}}^{\infty} \frac{\epsilon_{\nu^{\prime}}(r) d r^{2}}{\left(r^{2}-b^{2}\right)^{1 / 2}}
$$

where $\epsilon_{\nu}$ is the X-ray emissivity of the gas integrated over all angles, $\nu$ is the observed frequency, $\nu^{\prime}=(1+z) \nu$ is the emitted frequency, $z$ is the redshift, and $r$ is the physical distance from the cluster center.

We divided the image into $n$ annuli bounded by the angular radii $\theta_{0}<\theta_{1}<\cdots<$ $\theta_{i}<\theta_{i+1}<\cdots<\theta_{n}$ and centered on the peak of the cluster emission. In this chapter, we take $\theta_{0}=0$. Noting that $b=\theta d_{A}$, where $d_{A}$ is the angular diameter distance, the flux from the annulus from $\theta_{i} \rightarrow \theta_{i+1}$ is given by:

$$
F_{\nu}^{\mathrm{obs}}\left(\theta_{i} \rightarrow \theta_{i+1}\right)=\int_{\theta_{i}}^{\theta_{i+1}} I_{\nu}^{\mathrm{obs}}(\theta) 2 \pi \theta d \theta=\frac{\pi}{d_{A}^{2}} \int_{b_{i}^{2}}^{b_{i+1}^{2}} I_{\nu}^{\mathrm{obs}}(b) d b^{2}
$$

Putting equation (A.1) into (A.2), discretizing the integrals into summations, and assuming a constant emissivity $\left(\epsilon_{\nu^{\prime}, j}\right)$ within each radius $\left(b_{j} \rightarrow b_{j+1}\right)$, the observed flux can be expressed as: 


$$
\begin{aligned}
F_{\nu}^{\mathrm{obs}}\left(\theta_{i} \rightarrow \theta_{i+1}\right)= & \frac{1}{3(1+z)^{3} d_{A}^{2}}\left\{\epsilon_{\nu^{\prime}, i}\left(b_{i+1}^{2}-b_{i}^{2}\right)^{3 / 2}\right. \\
+ & \sum_{j=i+1}^{n-1} \epsilon_{\nu^{\prime}, j}\left[\left(b_{j+1}^{2}-b_{i}^{2}\right)^{3 / 2}-\left(b_{j}^{2}-b_{i}^{2}\right)^{3 / 2}\right. \\
& \left.\left.-\left(b_{j+1}^{2}-b_{i+1}^{2}\right)^{3 / 2}+\left(b_{j}^{2}-b_{i+1}^{2}\right)^{3 / 2}\right]\right\}
\end{aligned}
$$

or in matrix form:

$$
F_{\nu}^{\mathrm{obs}}\left(\theta_{i} \rightarrow \theta_{i+1}\right)=\sum_{j=0}^{n-1} A_{i j} \epsilon_{\nu^{\prime}, j}
$$

where $A_{i j}$ contains the geometrical factors in equation (A.3). The matrix $A_{i j}$ is triangular, and equation (A.4) can easily be inverted to give the emissivities at each radius. Once the emissivity is known, it can then be converted to the electron density, $n_{e}$, by the relation:

$$
\epsilon_{\nu}=n_{e}^{2} \Lambda_{\nu}
$$

where $\Lambda_{\nu}$ is the emissivity function which depends on the temperature and the abundances. Note that $\Lambda_{\nu}$ can be derived from the models used to fit the X-ray spectra. 


\section{Appendix B Cooling Time Estimation}

The cooling time of a region can be estimated from the normalization of the MEKAL model fitted by XSPEC, together with the fitted temperature, the bolometric luminosity estimated by XSPEC, and the deprojected density. The normalization of the MEKAL model in XSPEC is given by:

$$
K=\frac{10^{-14}}{4 \pi d_{A}^{2}(1+z)^{2}} \int n_{H} n_{e} d V \mathrm{~cm}^{-5}
$$

where $d_{A}$ and $z$ are the angular diameter distance and the redshift to the source, respectively, $n_{e}$ and $n_{H}$ are the electron and hydrogen densities, respectively, and $V$ is the volume of the region. It is easy to show that the enthalpy $H$ of the region is:

$$
\begin{aligned}
H & =\frac{5}{2} P V \\
& \approx \frac{2.5 \times 2.33 \times 4 \pi d_{A}^{2}(1+z)^{2} 10^{14} K}{n_{e}} k T \text { ergs } .
\end{aligned}
$$

The instantaneous isobaric cooling time is then given by:

$$
t_{\mathrm{cool}}^{*}=\frac{H}{L_{\mathrm{bol}}},
$$

where $L_{\mathrm{bol}}$ is the bolometric luminosity of the region which is given by XSPEC. The bolometric luminosity can be written as $L_{\mathrm{bol}}=\Lambda(T) n_{e}^{2} V$, where the emissivity function $\Lambda(T)$ depends on the abundances and is taken from the same XSPEC model which best fits the spectrum. Then, the integrated cooling time is

$$
t_{\mathrm{cool}}=t_{\mathrm{cool}}^{*} \frac{\Lambda(T)}{T^{2}} \int_{0}^{T} \frac{T^{\prime} d T^{\prime}}{\Lambda\left(T^{\prime}\right)} \approx \frac{t_{\mathrm{cool}}^{*}}{2}
$$


Table 2.1. XMM-Newton X-ray Point Sources with NED Identifications

\begin{tabular}{llllllll}
\hline \hline X-ray RA & X-ray Dec & \multicolumn{1}{c}{ Possible NED Object } & \multicolumn{1}{c}{ Type $^{\mathrm{a}}$} & NED RA & NED Dec & Redshift $^{\text {Se. }}$ \\
\hline 23:36:04.8 & $+21: 06: 11.2$ & 2MASX J23360455+2106127 & G/IrS & $23: 36: 04.6$ & $+21: 06: 13$ & 0.066130 & $3^{\prime \prime}$ \\
23:36:09.6 & $+21: 20: 25.1$ & MG3 J233615+2120 & RadioS & $23: 36: 09.7$ & $+21: 20: 25$ & $\ldots$ & $1^{\prime \prime}$ \\
23:36:15.8 & $+21: 06: 11.5$ & Abell 2626:[SPS89] 08 & VisS/RadioS & $23: 36: 16.2$ & $+21: 06: 21$ & $\ldots$ & $11^{\prime \prime}$ \\
23:36:24.5 & $+21: 09: 03.6$ & IC 5337 & G/IrS/RadioS & $23: 36: 25.1$ & $+21: 09: 02$ & 0.054988 & $9^{\prime \prime}$ \\
23:36:30.5 & $+21: 08: 47.0$ & IC 5338 & G/IrS/RadioS & $23: 36: 30.6$ & $+21: 08: 50$ & 0.054900 & $3^{\prime \prime}$ \\
$23: 36: 53.8$ & $+21: 13: 32.9$ & 2MASX J23365372+2113322 & G/IrS & $23: 36: 53.7$ & $+21: 13: 32$ & 0.056100 & $2^{\prime \prime}$ \\
$23: 36: 54.2$ & $+21: 15: 31.7$ & 2MASX J23365415+2115302 & G/IrS & $23: 36: 54.2$ & $+21: 15: 29$ & 0.054200 & $3^{\prime \prime}$ \\
23:36:57.1 & $+21: 14: 05.6$ & 2MASX J23365722+2114032 & G/IrS & $23: 36: 57.2$ & $+21: 14: 03$ & 0.038040 & $3^{\prime \prime}$ \\
$23: 37: 32.4$ & $+21: 09: 07.6$ & 2MASX J23373222+2109020 & G/IrS & $23: 37: 32.2$ & $+21: 09: 02$ & 0.184400 & $6^{\prime \prime}$ \\
\hline
\end{tabular}

a The source type as classified by NED: G=Galaxies; IrS=Infrared Source; RadioS=Radio Source; VisS $=$ Visual Sources.

b Separation between the X-ray point source and source identified by NED.

Table 2.2. Chandra X-ray Point Sources

\begin{tabular}{cccc}
\hline \hline Point Source & RA & Dec & NED Object \\
\hline 1 & $23: 36: 15.82$ & $+21: 08: 55.4$ & $\ldots$ \\
2 & $23: 36: 21.31$ & $+21: 13: 29.7$ & $\ldots$ \\
3 & $23: 36: 21.41$ & $+21: 11: 35.4$ & $\ldots$ \\
4 & $23: 36: 24.61$ & $+21: 08: 47.7$ & $\ldots$ \\
5 & $23: 36: 24.81$ & $+21: 10: 16.7$ & $\ldots$ \\
6 & $23: 36: 25.04$ & $+21: 09: 03.0$ & IC 5337 \\
7 & $23: 36: 25.60$ & $+21: 13: 07.7$ & $\ldots$ \\
8 & $23: 36: 30.52$ & $+21: 08: 47.0$ & IC 5338 \\
9 & $23: 36: 33.30$ & $+21: 08: 36.7$ & $\ldots$ \\
10 & $23: 36: 36.98$ & $+21: 10: 51.8$ & $\ldots$ \\
11 & $23: 36: 37.46$ & $+21: 06: 21.0$ & $\ldots$ \\
12 & $23: 36: 38.43$ & $+21: 08: 26.1$ & $\ldots$ \\
13 & $23: 36: 45.60$ & $+21: 07: 49.3$ & $\cdots$ \\
14 & $23: 36: 46.84$ & $+21: 08: 07.8$ & $\ldots$ \\
\hline
\end{tabular}



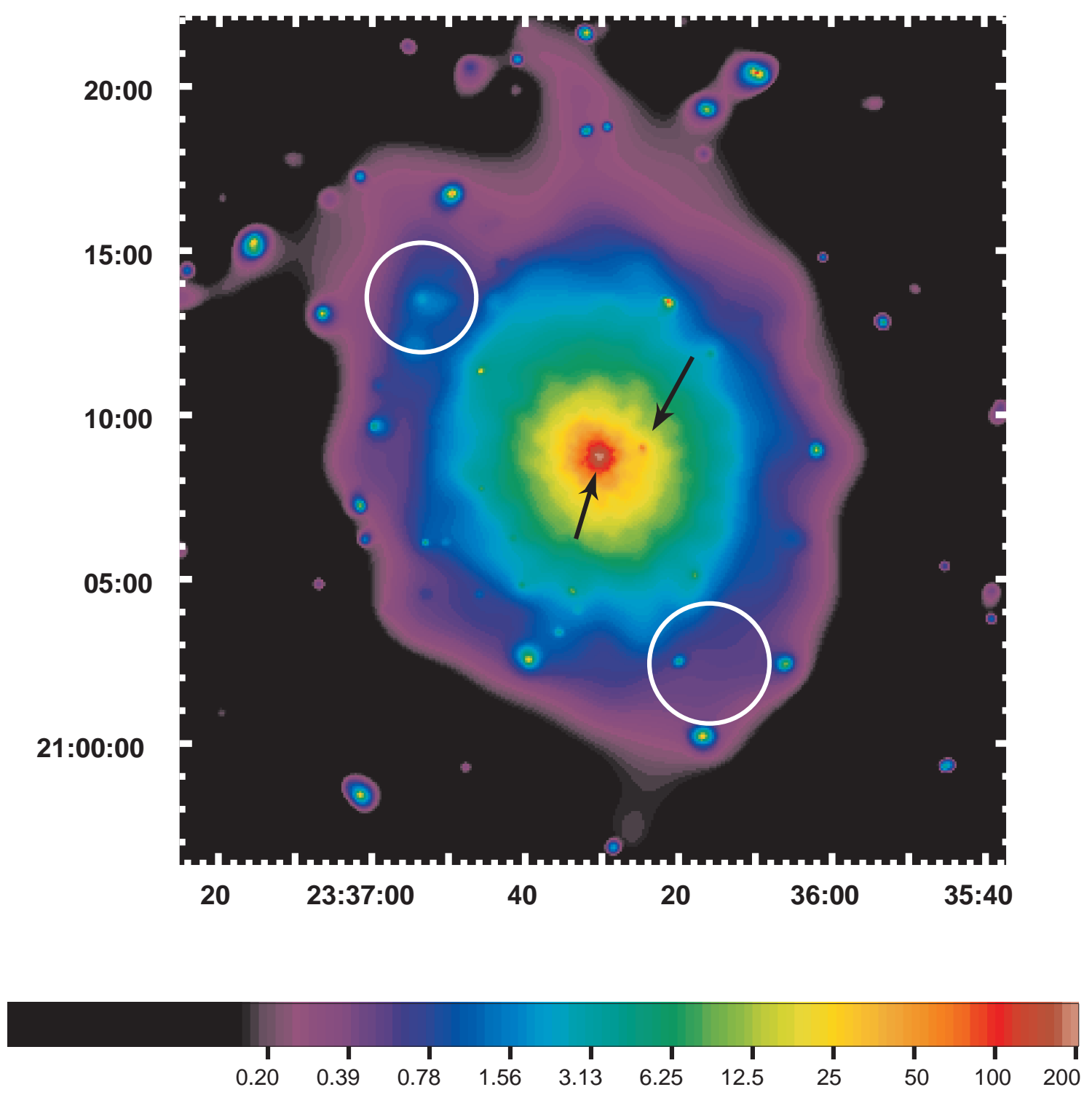

Fig. 2.1. - Background-subtracted, exposure-corrected, adaptively smoothed mosaic of the XMM-Newton EPIC MOS1 and MOS2 images in the $0.3-10 \mathrm{keV}$ band. The image was smoothed to a signal-to-noise ratio of 3 . The units for the color scale are counts per binned pixel, where each binned pixel has a size of 4 "' $1 \times 44^{\prime \prime} 1$. The arrows on the left and right indicate the cD galaxy IC 5338 and the S0 galaxy IC 5337, respectively. Two outer extended emission regions are indicated with white circles. It should be noted that there is a chip gap at about 5'5 from the center which makes the northeast extended X-ray source appear to be more distinct than it actually is. 

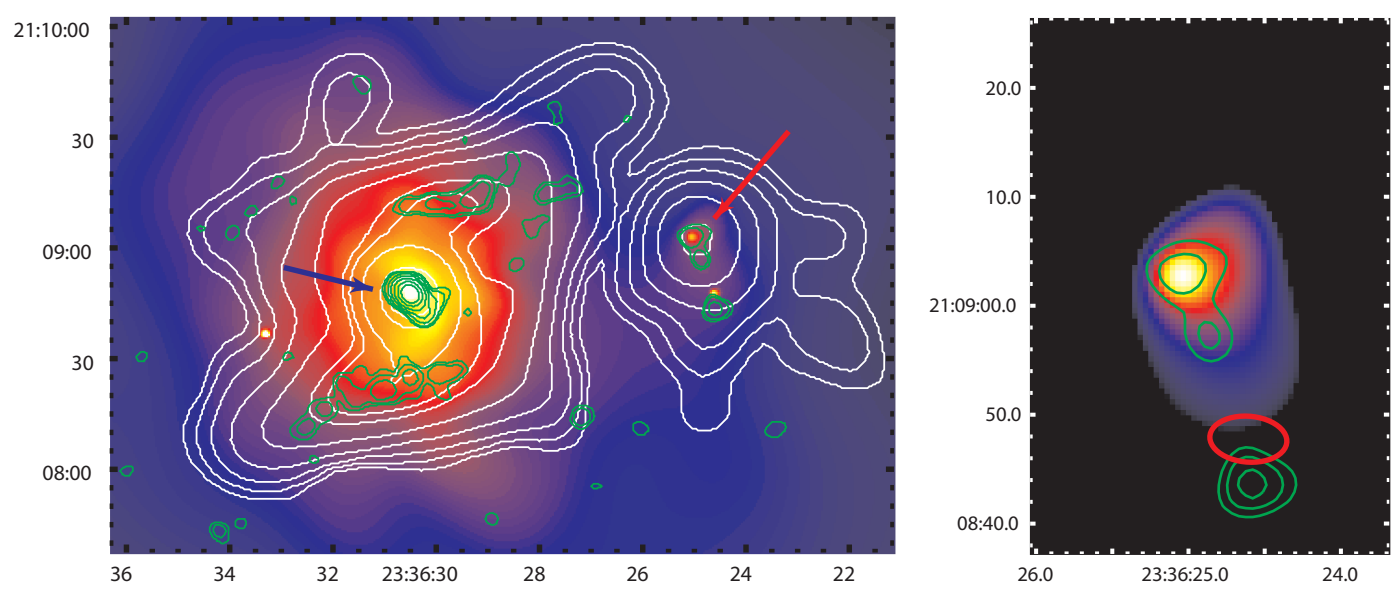

Fig. 2.2.- Left panel: Background subtracted, exposure-corrected, adaptively smoothed Chandra image of the central 3.5 $\times 2$ '.4 region of the cluster in the $0.3-$ $10 \mathrm{keV}$ band. The image was smoothed to a signal-to-noise ratio of 3 . The Chandra image shows the details of the inner region of the cooling core. The white solid lines are $1.5 \mathrm{GHz}$ C-array radio contours showing the mini-halo. The green solid lines are $1.5 \mathrm{GHz}$ B-array radio contours showing the radio bar structures. Both radio contours were taken from Gitti et al. (2004). The arrows on the left and right indicate the cD galaxy IC 5338 and the S0 galaxy IC 5337, respectively. Right panel: Background subtracted, exposure-corrected, adaptively smoothed Chandra image centered on the S0 galaxy IC 5337. The image was smoothed to a signal-to-noise ratio of 4, with an intensity scale chosen to show the bow-shock-like shape. The southern source (red ellipse) was removed and replaced by the average surrounding X-ray intensity before adaptively smoothing. The green contours are from the VLA $1.5 \mathrm{GHz}$ B-array radio image taken from Gitti et al. (2004), with the lowest contour level being a signal-to-noise ratio of 3 . 


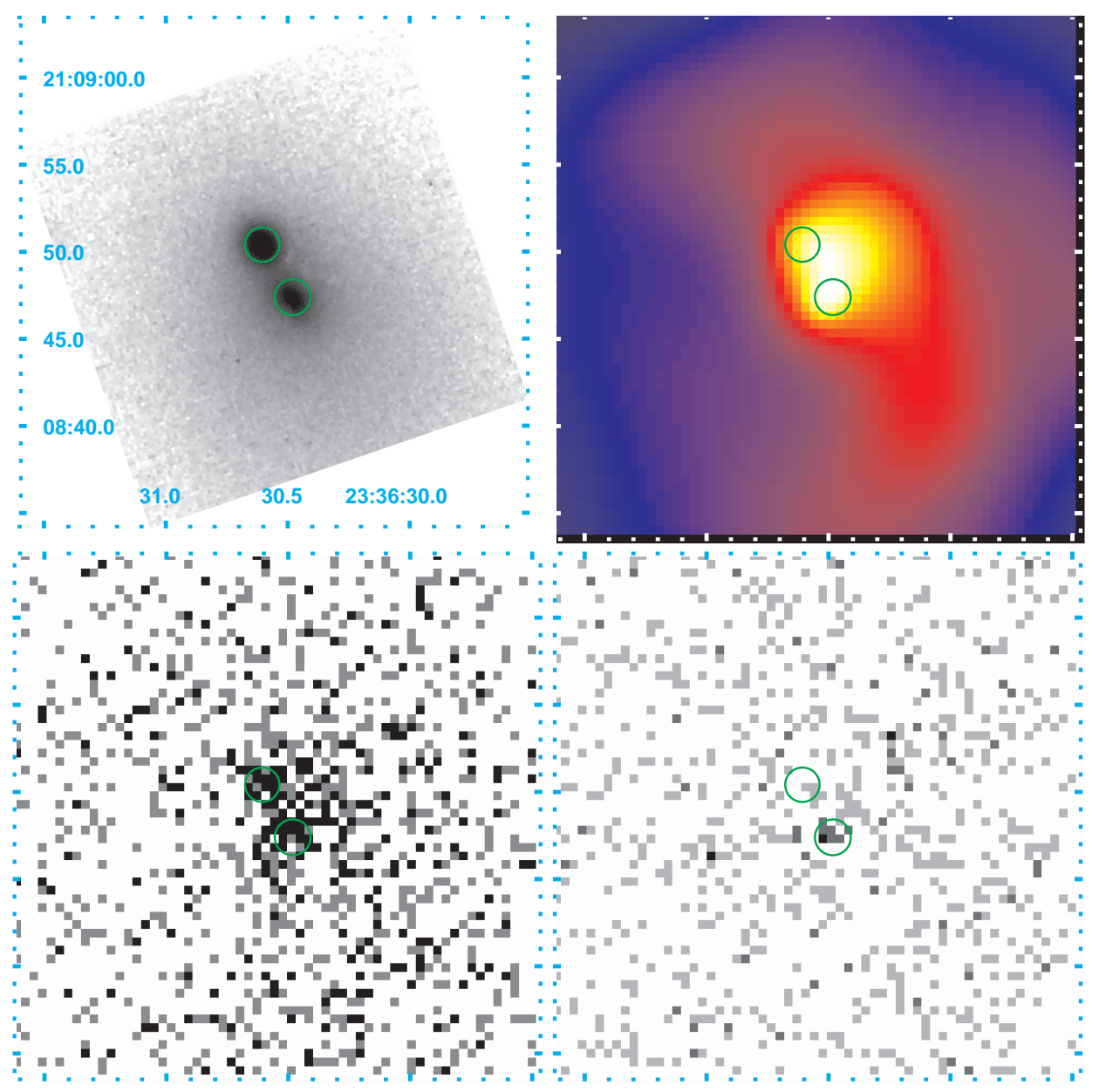

Fig. 2.3. - Images of the two nuclei of the cD galaxy IC 5338 in Abell 2626. All images show the same field of view of $30^{\prime \prime} \times 30^{\prime \prime}$. Upper left panel: Optical image from HST archive. The image was taken with WFPC2 using the F555W filter. The two green circles, included in the other panels, are centered at the two optical nuclei observed by HST. Upper right panel: Background-subtracted, exposure-corrected, adaptively smoothed Chandra image in $0.3-10 \mathrm{keV}$ band. The image was smoothed to a signal-to-noise ratio of 3 . The color represents the X-ray intensity from high (white yellow) to low (dark blue). Lower left panel: Raw Chandra image in the soft (0.3-2 keV) band. Two intensity peaks can be identified. Lower right panel: Raw Chandra image in the hard $(2-10 \mathrm{keV})$ band. Only the southwest nucleus corresponds to the peak in the hard band. 


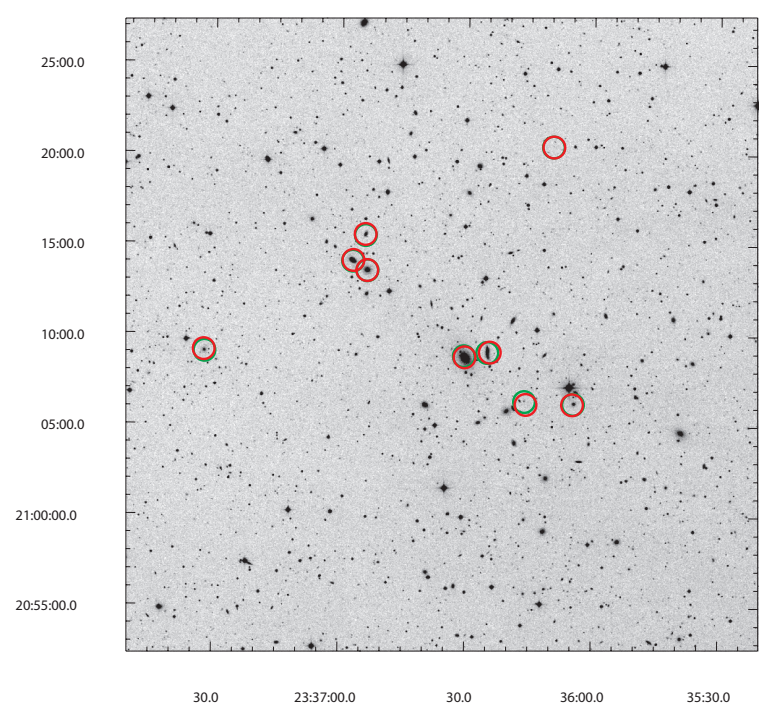

Fig. 2.4.- Optical image from the Digital Sky Survey showing the 9 XMM-Newton $\mathrm{X}$-ray sources with identifications in NED (Table 2.1). The red circles are centered on the X-ray positions determined with $X M M$-Newton. There are green circles centered on the optical positions from NED, but they overlap so completely with the red circles that they are barely visible. NED objects are considered possible identifications if they are within $20^{\prime \prime}$ of an X-ray source. In fact all optical counterparts identified are within $11^{\prime \prime}$.

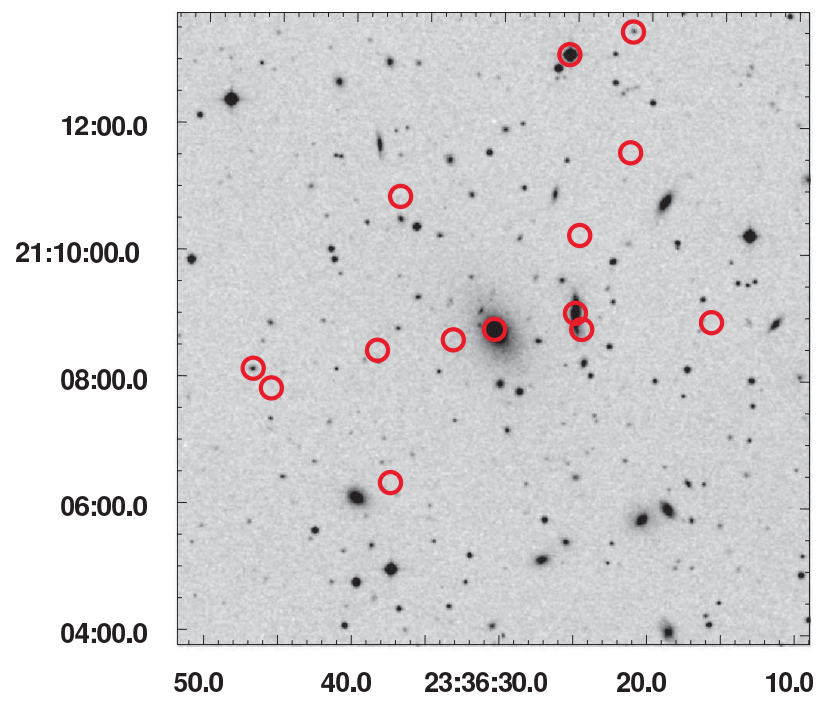

Fig. 2.5.- Optical image from the Digital Sky Survey showing the positions (red circles) of the 14 X-ray point sources detected with Chandra (Table 2.2). 

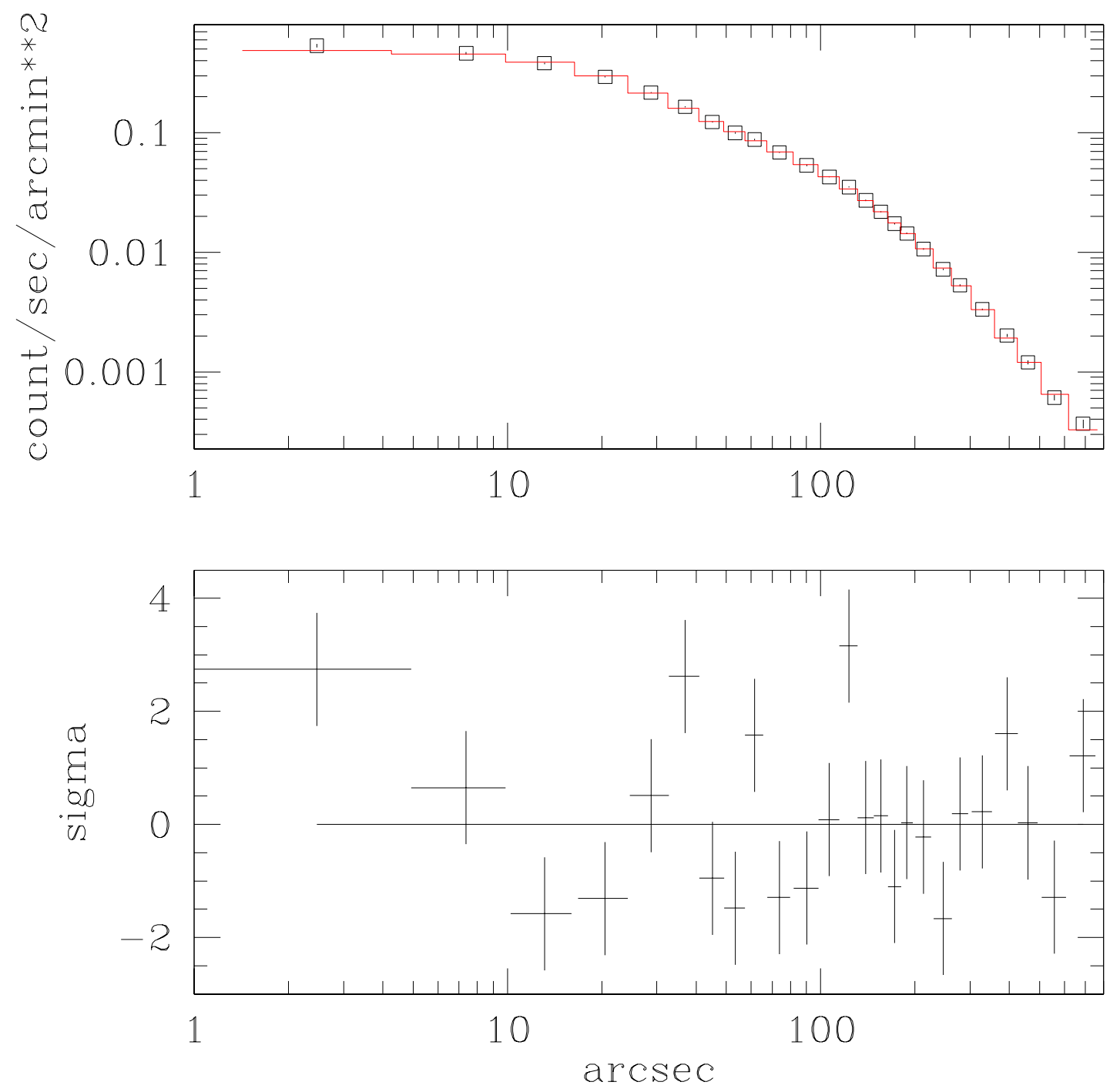

Fig. 2.6.- Upper panel: Azimuthally averaged X-ray surface brightness profile from the XMM-Newton MOS1 and MOS2 observations of Abell 2626. The squares are the $\mathrm{X}$-ray data, while the solid line is the fit to a double $\beta$ model. The tiny error bars within the squares on the surface brightness are too small to be easily seen. Lower panel: The residuals to the best fit double beta model, in unit of $\sigma$. The horizontal error bars show the widths of the bins used to accumulate the counts. The vertical error bars are at the $1 \sigma$ level. 

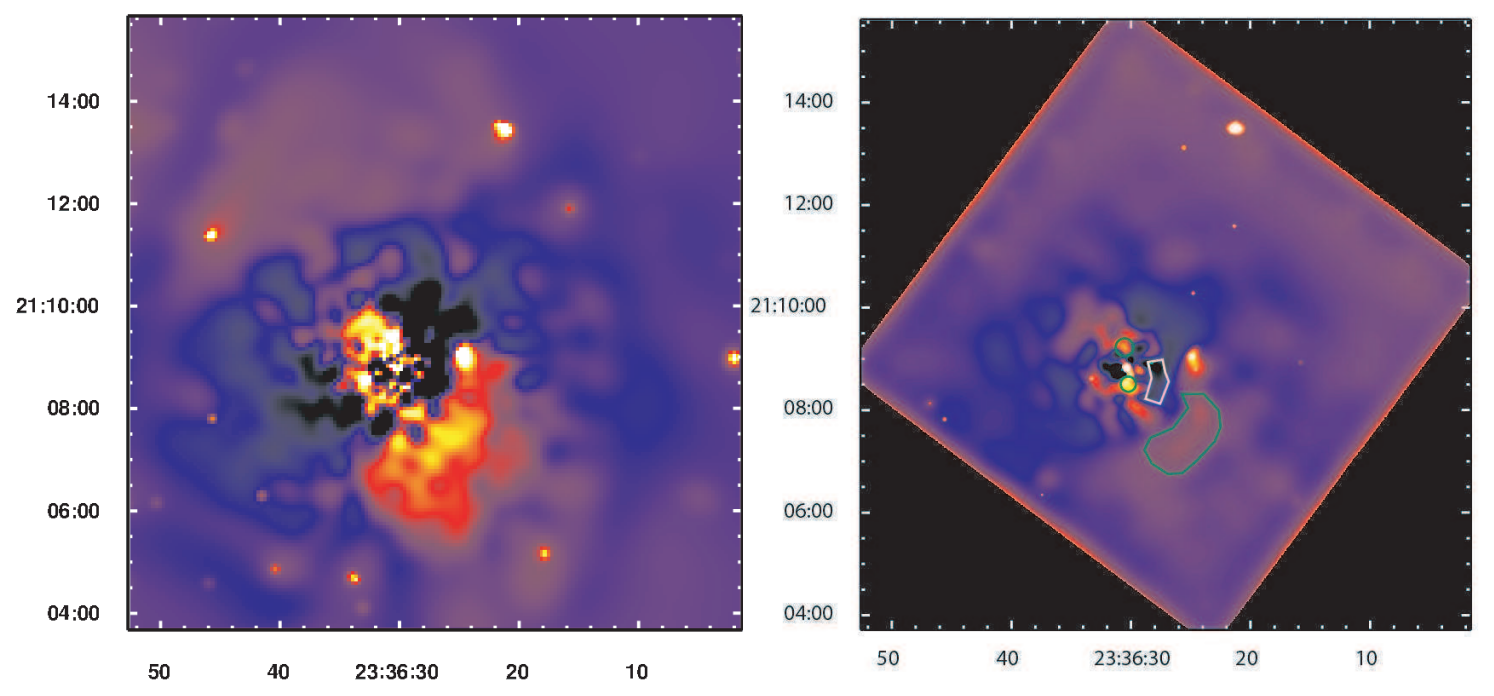

Fig. 2.7. - Left panel: Residual map from the XMM-Newton MOS1 and MOS2 observations of Abell 2626, generated from a background-subtracted image. Yellow represents the largest excess, while black is the greatest deficit. Right panel: Residual map from the Chandra observation of Abell 2626, generated from a backgroundsubtracted image. Yellow represents the largest excess, while black is the greatest deficit. There is an excess coincident with the center of the central cD galaxy, which is partly surrounded by a trough of reduced X-ray surface brightness at a radius of about $15^{\prime \prime}$. There are two localized regions of excess south and north of the central peak, which are indicated with green circles. There is an extended deficit about $50^{\prime \prime}$ west of the center, which is indicated with a white polygon. An extended region of excess emission is seen about $110^{\prime \prime} \mathrm{SW}$ of the center, which is indicated with a green polygon. 


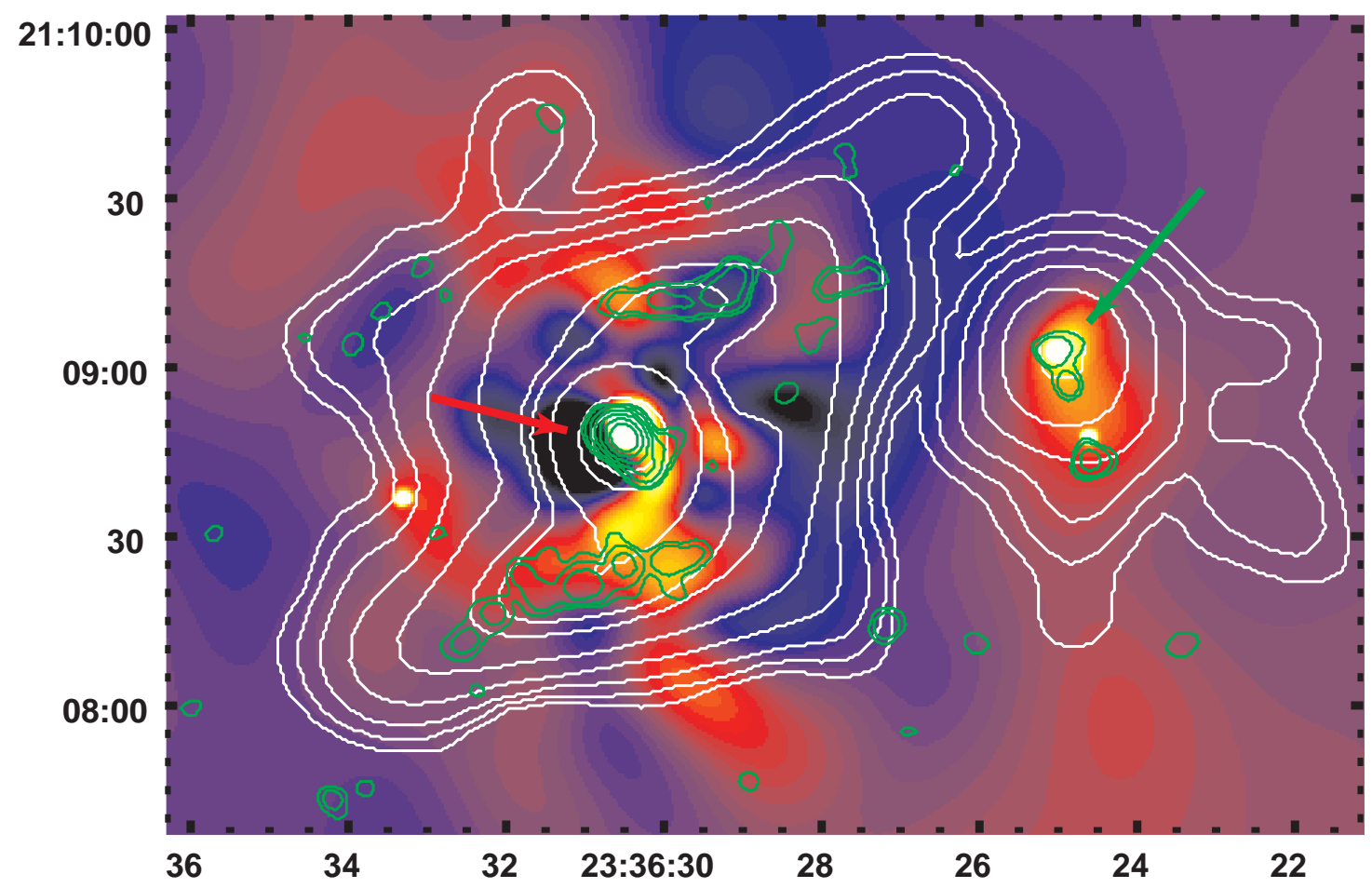

Fig. 2.8. - Residual map from Chandra observation of the central 3'.5 $\times 2$ ' 4 region of Abell 2626, generated from a background-subtracted image. The color scale from yellow to red to blue to black represents the residuals, running from excess to deficit. The white solid lines are $1.5 \mathrm{GHz} \mathrm{C}$-array radio contours showing the mini-halo. The green solid lines are $1.5 \mathrm{GHz}$ B-array radio contours showing the radio bar structures. Both radio contours were taken from Gitti et al. (2004). The arrows on the left and right indicate the $\mathrm{cD}$ galaxy IC 5338 and the S0 galaxy IC 5337, respectively. 


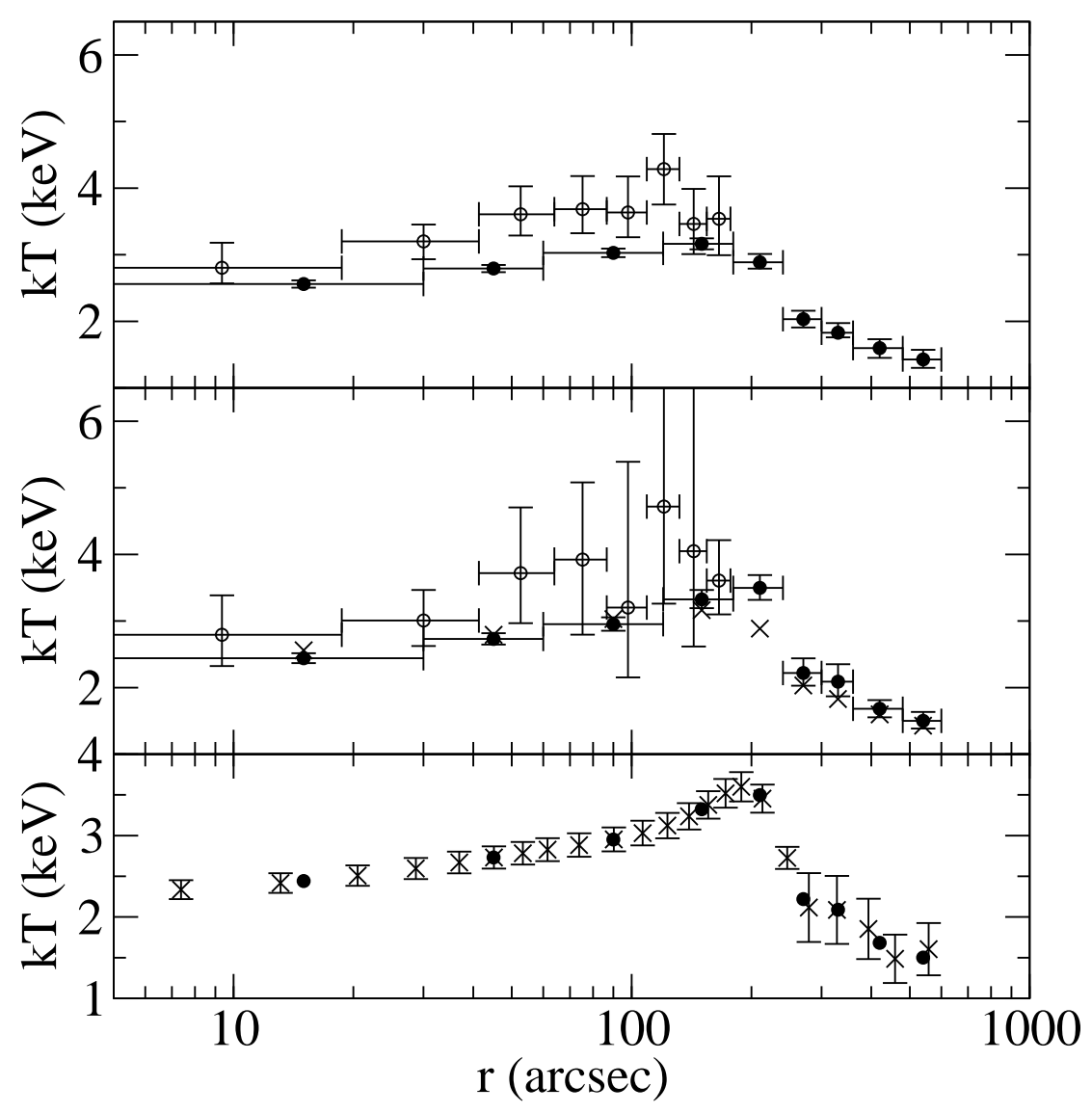

Fig. 2.9.- Upper Panel: Profile of the azimuthally averaged, projected temperature $T$ of Abell 2626. The XMM-Newton (Chandra) data are shown as solid (open) circles. Middle Panel: Profile of the azimuthally averaged, deprojected temperature $T$ of Abell 2626. The XMM-Newton (Chandra) data are shown as solid (open) circles. The black crosses are the projected temperatures from the XMM-Newton data in the upper panel for comparison (without error bars for clarity). In both the upper and middle panels, the error bars are at the 90\% confidence level. Lower Panel: Crosses are the temperatures calculated from interpolation of the original XMM-Newton deprojected temperature profile; this interpolated profile was used for the pressure/entropy/time scales deprojection and the mass profile analysis in Sections 2.4.3, 2.4.4, \& 2.4.5. The interpolated temperature profile for the Chandra data analysis is similar but not shown for clarity. The errors are assigned as 5 (20)\% at radii of smaller (larger) than $250^{\prime \prime}$ to include possible systematic errors. The solid circles are the original XMMNewton measured deprojected temperatures as shown in the middle panel, without error bars for clarity. 


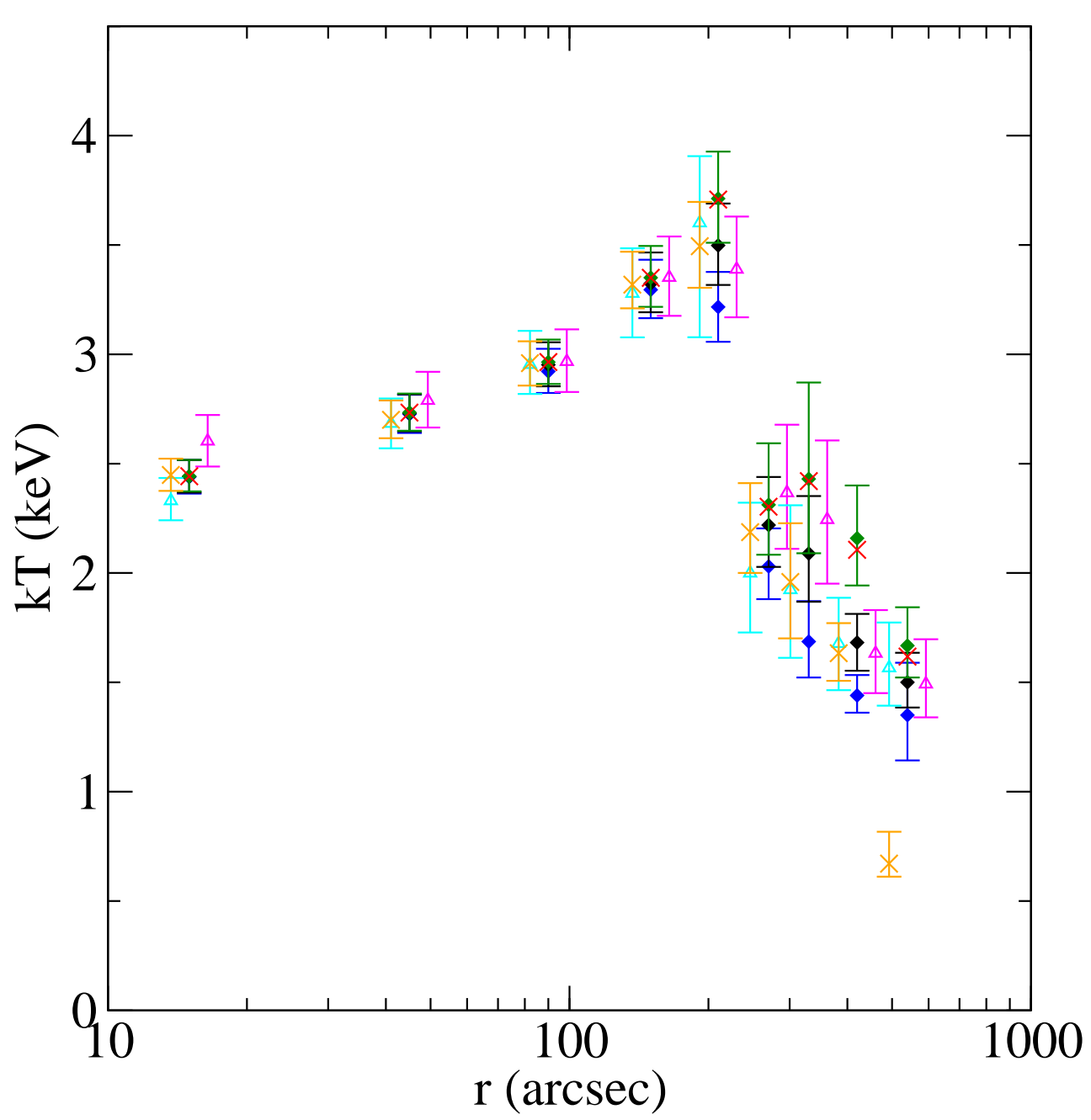

Fig. 2.10. - Profile of the azimuthally averaged, deprojected temperature $T$ of Abell 2626 with different XMM-Newton backgrounds. The black diamonds give our adopted XMM-Newton temperature profile (the black solid circles in the second panel of Figure 2.9). The other temperature profiles are generated by: 1a) (blue diamonds) increasing the blank-sky background normalizations by $10 \%$ (20\%) for MOS (PN); 1b) (green diamonds) decreasing the blank-sky background normalizations by $10 \%$ (20\%) for MOS (PN); 2) (red crosses) using the blank-sky backgrounds without renormalization (no error bars for clarity); 3) (magenta triangles) using the MOS1+MOS2 spectra only (data shifted to the right for clarity); 4) (cyan triangles) using the PN spectra only (data shifted to the left for clarity); 5) (orange crosses) adding an extra $0.2 \mathrm{keV}$ MEKAL component to represent variations in the soft Galactic component (normalization free and allowed to be negative in each annulus, data shifted to the left for clarity). The error bars are at the $90 \%$ confidence level. 


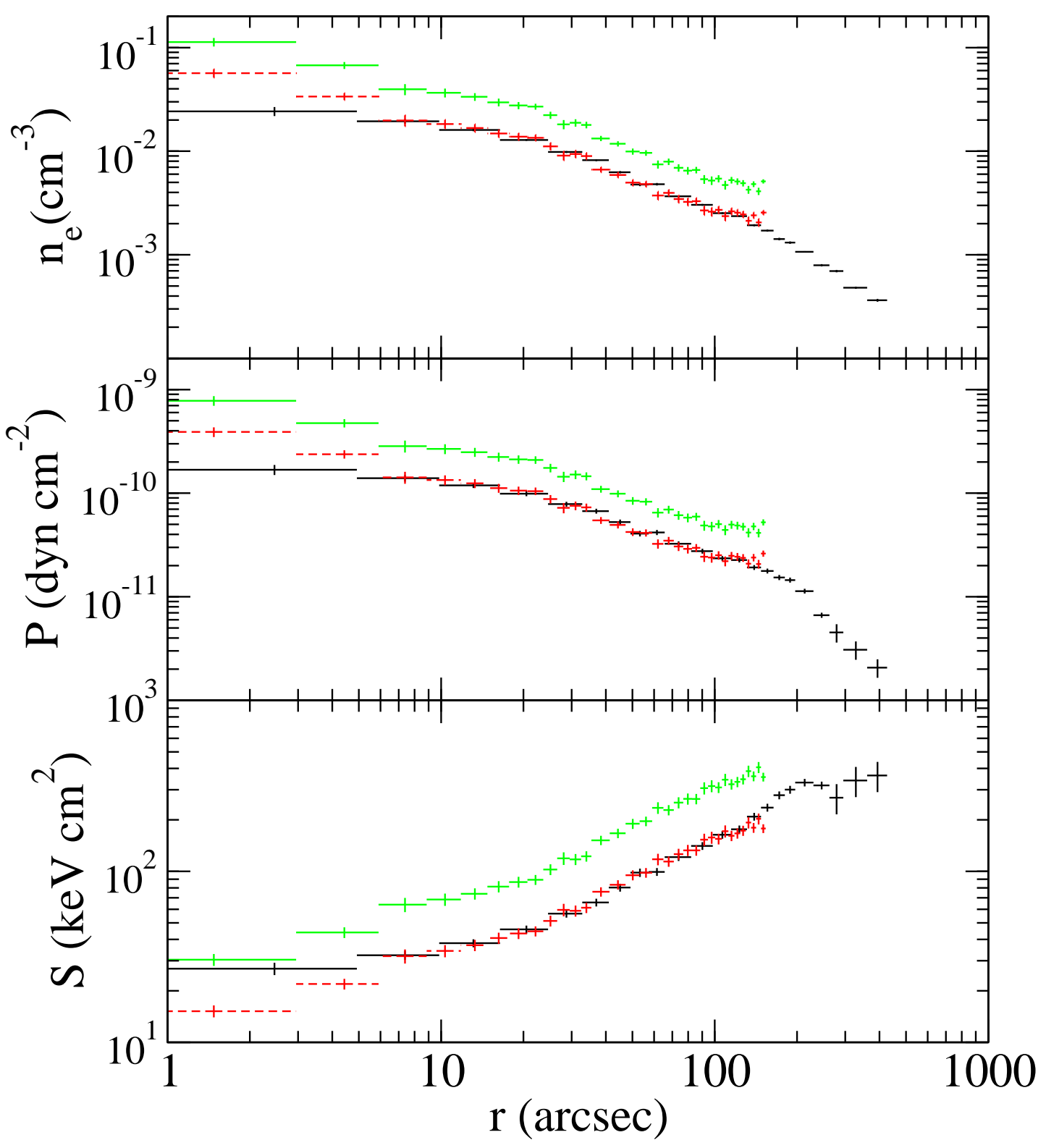

Fig. 2.11.- Upper Panel: Azimuthally averaged electron density profile of Abell 2626. The XMM-Newton (Chandra) data are shown as black solid (red dashed) symbols. For clarity, we have also multiplied the Chandra data and error bars by a factor of 2 (green solid symbols). The error bars are at the $1 \sigma$ level. Middle Panel: Azimuthally averaged pressure profile, with the same notation as the upper panel. Lower Panel: Azimuthally averaged entropy profile, with the same notation as the upper panel. 


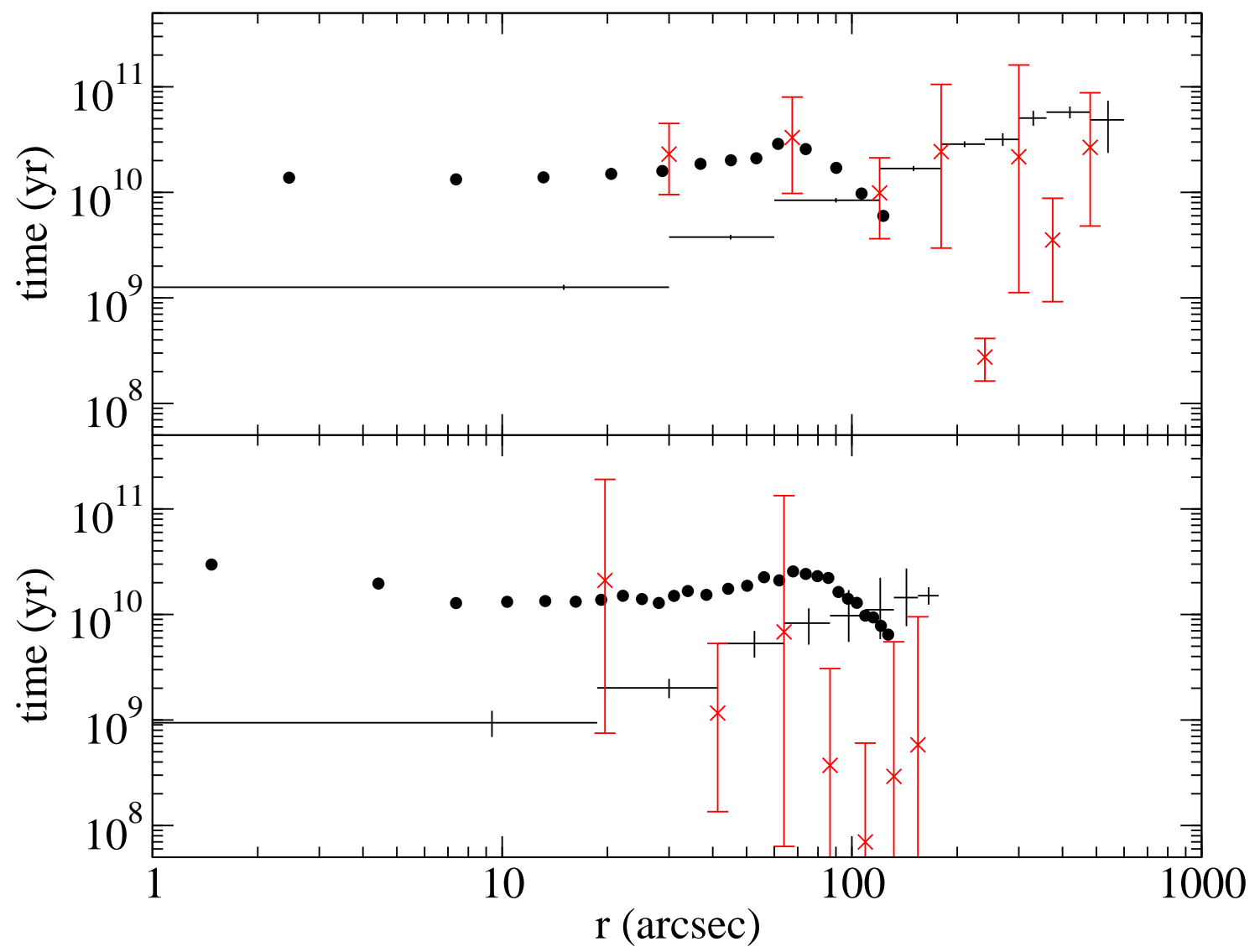

Fig. 2.12.- Upper Panel: Cooling and thermal conduction time scales in Abell 2626 obtained from the XMM-Newton data. The cooling times are the plus symbols with horizontal bars showing the annular regions sampled. The conduction time scales derived directly from individual data points are the crosses with larger vertical error bars. The dots are conduction time scales calculated by interpolating the temperature profile of the cluster (see text in Section 2.4.4). The radial regions for the conduction time are not given for clarity. The error bars are at the $1 \sigma$ level. Lower Panel: Same as the upper panel, but with the time scales calculated from the Chandra data. 


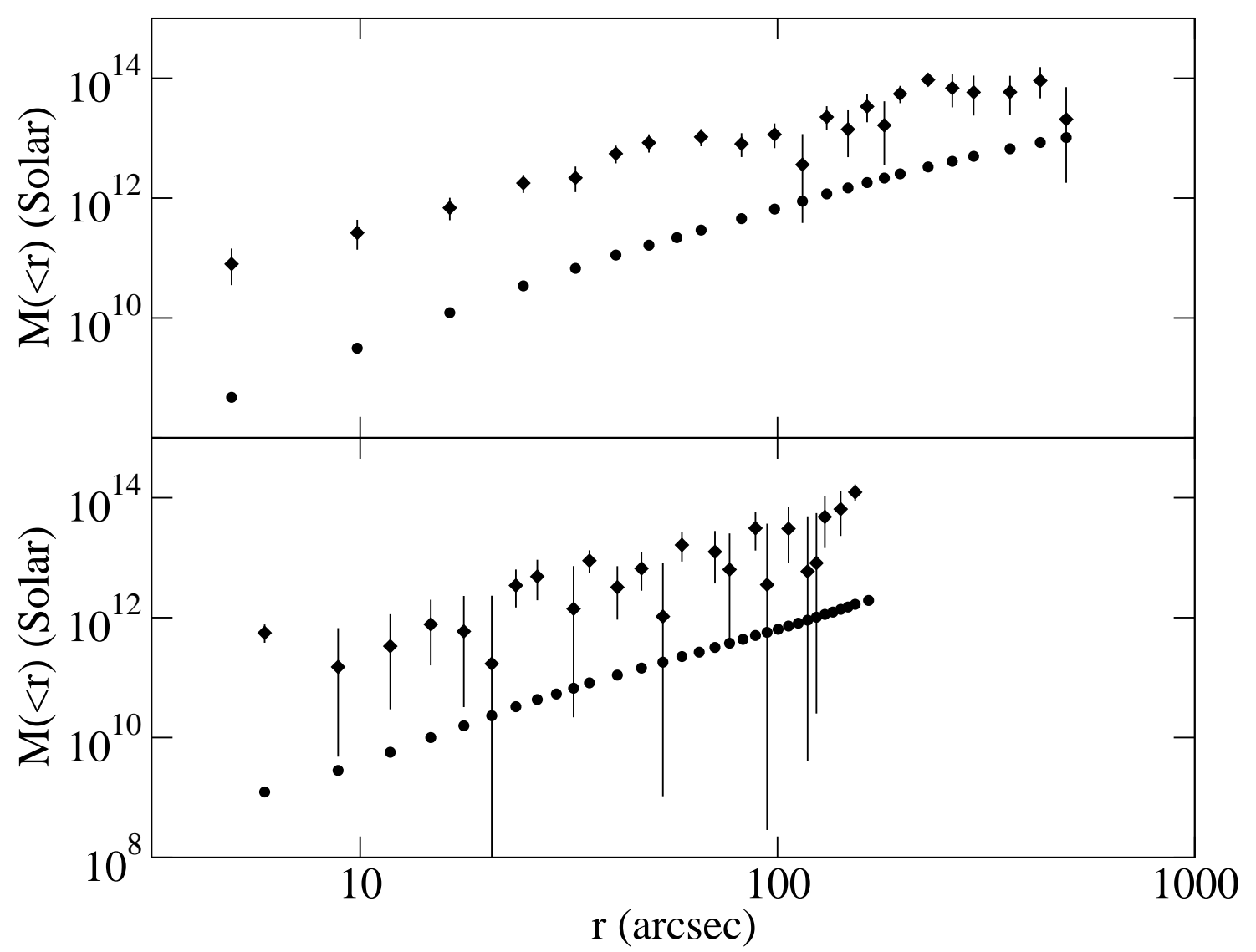

Fig. 2.13.- Upper panel is the interior mass profile $[M(<r)]$, of Abell 2626 obtained from XMM-Newton data, while the lower panel is from Chandra data. The total mass is shown with diamond symbols, while the gas mass points are circles. The error bars on the masses are calculated using propagation of errors at $1 \sigma$ level. The error bars on the gas mass are plotted, but are smaller than the circle symbols. 


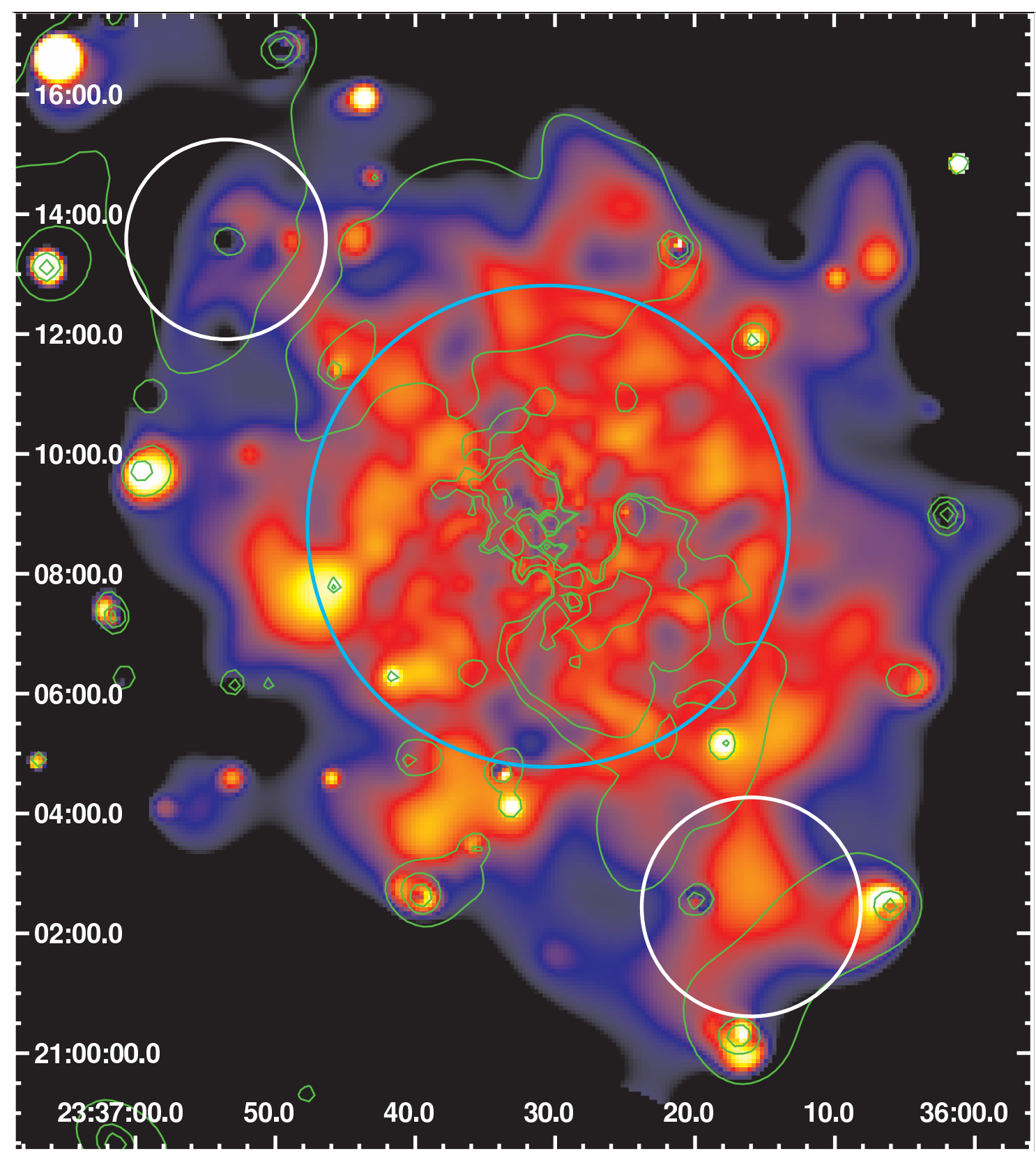

Fig. 2.14. - Hardness ratio $(H R)$ map of Abell 2626 from the XMM-Newton observation. Background was subtracted from the images combined to calculate the hardness ratio. The color scale ranges from black $(H R \sim 0)$ to blue $(H R \sim 0.1)$ to red $(H R \sim 0.3)$ to yellow $(H R \sim 0.5)$ to white $(H R \sim 2.5)$, showing increasingly hard emission. The green solid lines are contours from XMM-Newton residual map (left panel of Figure 2.7) showing positive residuals (excess emission). The white circles are the regions of extended X-ray emissions to the northeast and southwest. The cyan circle has a radius of $240^{\prime \prime}$ corresponding to the jump in the temperature profile. 

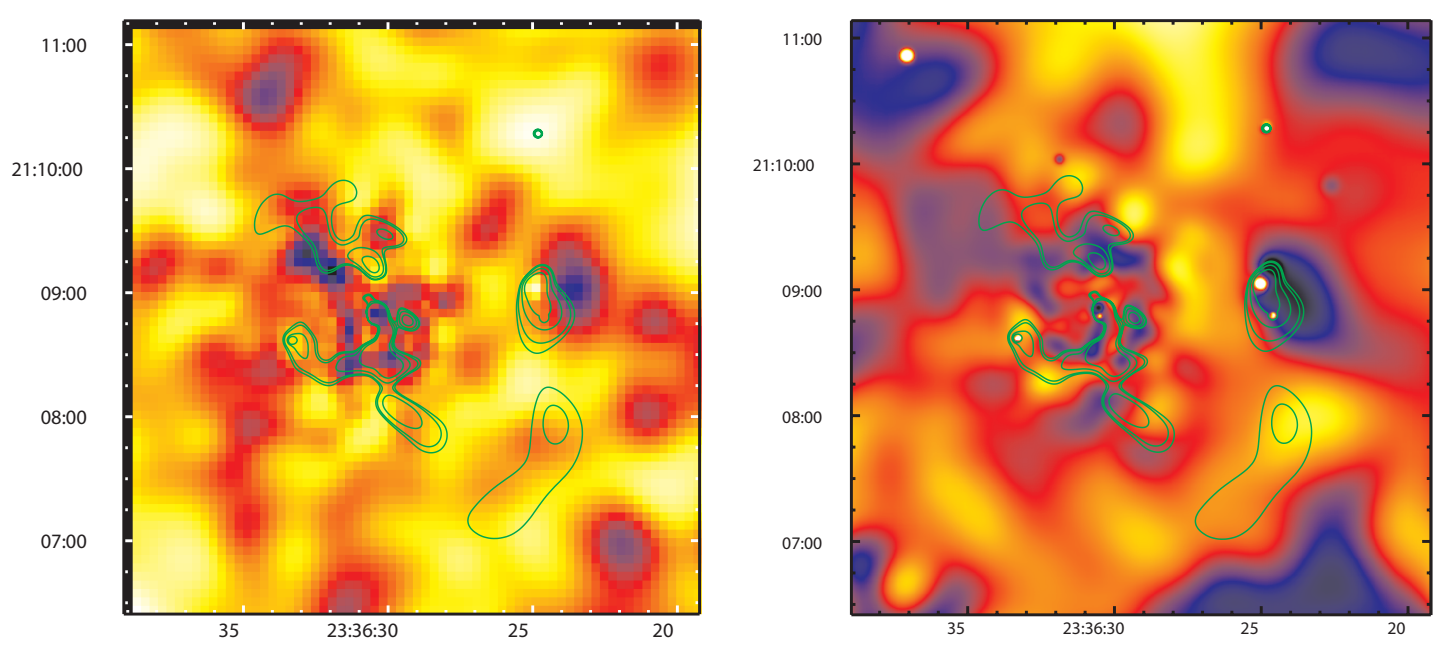

Fig. 2.15. - Left panel: Central portion $\left(4^{\prime} .6 \times 4.7\right)$ of the XMM-Newton hardness ratio map (Figure 2.14). The color scale is similar to that in Figure 2.14, but with the hardness ratio extremes being $\sim 0.2$ and 0.35 (dark blue to white yellow). The green solid lines are contours from the Chandra residual map (right panel of Figure 2.7) showing the excess emission. Right panel: Hardness ratio map from the Chandra data, for the same regions and with the same contours as the left panel. Background was subtracted from the images combined to calculate the hardness ratio. The color scale is similar to that in Figure 2.14, but with the hardness ratios in the range from $\sim 0.1$ to 1.5 (dark blue to white yellow). The cD galaxy IC 5338 and the S0 galaxy IC 5337 are located at (23:36:31, $+21: 08: 47)$ and (23:36:25, $+21: 09: 03)$, respectively. 


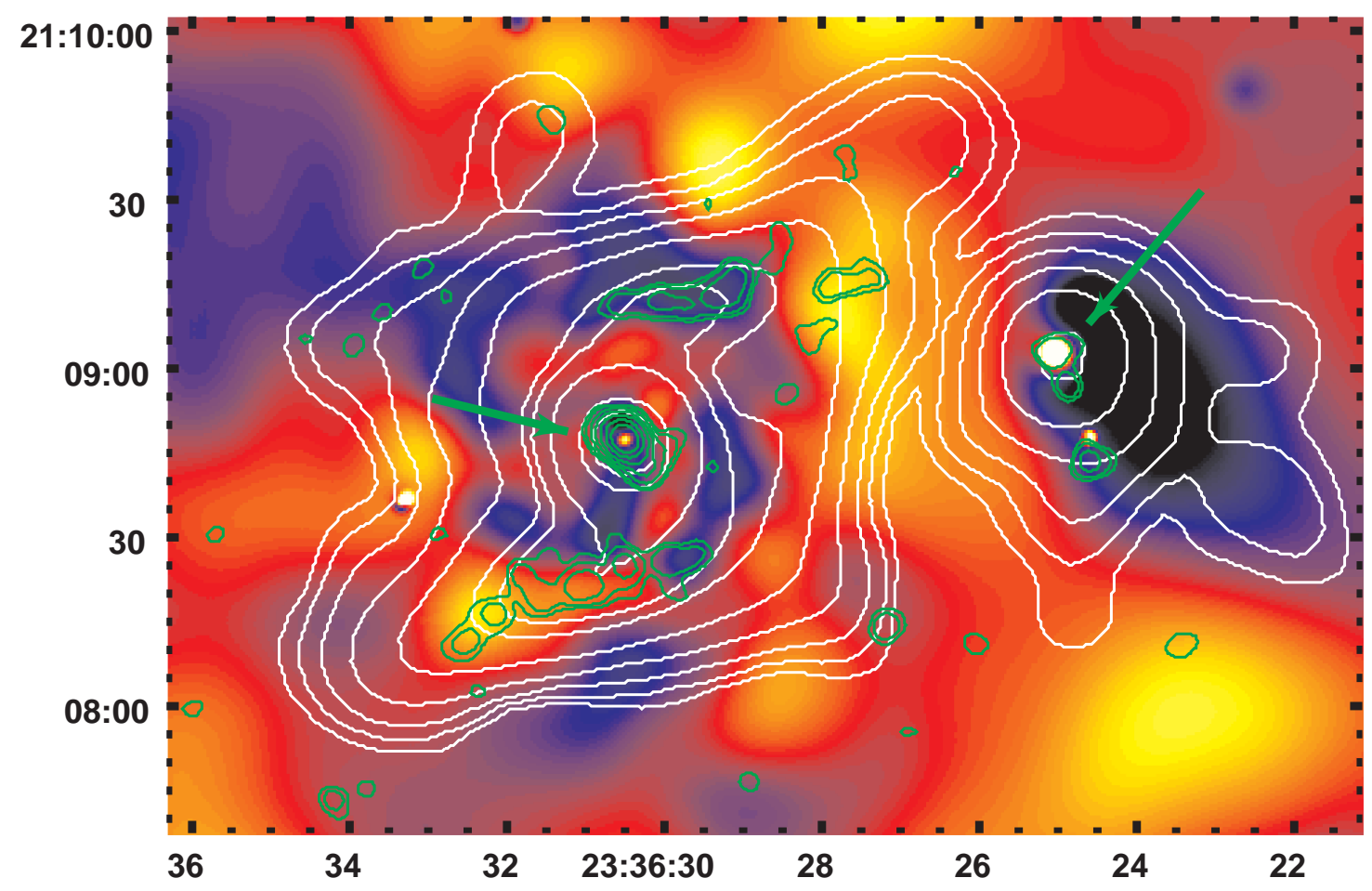

Fig. 2.16. - Central region $\left(3 \prime .5 \times 2{ }^{\prime} 4\right)$ of the hardness ratio map from the Chandra observation. Background was subtracted from the images combined to calculate the hardness ratio. The color scale is roughly the same as in the right panel of Figure 2.15. The white solid lines are $1.5 \mathrm{GHz} \mathrm{C}$-array radio contours showing the mini-halo. The green solid lines are $1.5 \mathrm{GHz}$ B-array radio contours showing the radio bar structures. Both radio contours were taken from Gitti et al. (2004). The arrows on the left and right indicate the $\mathrm{cD}$ galaxy IC 5338 and the S0 galaxy IC 5337, respectively. 


\section{Chapter 3}

\section{Effects of the Non-Equipartition of Electrons and Ions in the Outskirts of Relaxed Galaxy Clusters}

[Adapted from Wong, K.-W., \& Sarazin, C. L. 2009, ApJ, 707, 1141]

\subsection{Introduction}

Observational and theoretical studies have shown that the study of the intracluster medium (ICM) can be used as a test of plasma physics under extreme environments that cannot be achieved in terrestrial laboratories, as well as an important cosmological probe. If we assume the matter content of clusters is a fair sample of the universe, the baryon fraction of clusters can be used as an estimator of the average value for the universe, with proper correction for the baryons contained in the stellar component and for a small amount of baryonic matter expelled from clusters during the formation process. Recent work from (Allen et al. 2008) has shown that the combined results of 
the baryon fraction from X-ray observation with the cosmic microwave background (CMB) data can give powerful constraints on cosmological parameters, such as the equation of state of the dark energy.

However, the study of cosmology using clusters of galaxies relies heavily on the understanding of cluster physics. For precision cosmology, systematic uncertainties at even the percent level are significant. For example, in current studies, the baryon fraction within clusters is assumed to be independent of redshift and the mass of clusters. It would be important to see if these assumptions are justified; if not, it is important to study the dependence of the baryon fraction on cluster properties and redshift. Even if the dependence on redshift is weak, the correction factor for the baryon content within clusters compared to the average value in the universe could affect the constraints on cosmological parameters.

Studying cluster outskirts $\left(\gtrsim R_{200}{ }^{1}\right)$ is very important because the boundary conditions of the cluster outskirts constrain the global properties of a cluster (e.g., Tozzi et al. 2000). Also, the outer envelopes of clusters have been thought to be less subject to some additional physics including active galactic nucleus (AGN) feedback, and that the outer regions of clusters may provide better cosmological probes. Currently, there are very few observations of the properties of the ICM in the outer parts of clusters. Thus, most of our understanding of these regions is still based on numerical hydrodynamic simulations which assume the hot plasma is a fluid. In these simulations, the clusters are formed from mergers and accretion of dark matter and baryonic gas in overdense regions. A variety of shocks with different geometries along the large-scale structure (LSS) filaments and transverse to them near and beyond the virial radius are unambiguous predictions of the cosmological hydrodynamic simulations. Unfor-

\footnotetext{
${ }^{1} R_{\Delta}$ is the radius within which the mean total mass density of the cluster is $\Delta$ times the critical density. The virial radius $R_{\mathrm{vir}}$ is defined as a radius within which the cluster is virialized. For the Einstein-de Sitter universe, $R_{\mathrm{vir}} \approx R_{178}$, while for the standard $\Lambda$ CDM universe, $R_{\mathrm{vir}} \approx R_{95}$.
} 
tunately, the lack of observational information on the clusters' outskirts prevents us from understanding the accretion shock region, and hence the input physics for the numerical simulations is called into question. For example, the thermodynamic state of the shocked gas, as well as the shock position, depends on the pre-shock gas temperature; the shock will be weaker if the infalling gas is pre-heated (Tozzi et al. 2000). Even worse, recently it was noted that the non-fluid properties may be important in regions near the virial radius, where the Coulomb collisional mean free path is comparable to the cluster size of a few Mpc (Loeb 2007). The Coulomb collisional timescale can also be of the order of the age of the cluster. This suggests that a full kinetic gas theory is needed instead of the fluid approximation when studying the gas properties near the edge of the cluster. Direct consequences include non-equipartition between electrons and ions (Fox \& Loeb 1997; Ettori \& Fabian 1998), element sedimentation (Chuzhoy \& Loeb 2004), and suprathermal evaporation of hot gas from the clusters (Loeb 2007; see also Medvedev 2007). Some of these effects can lead to a bias in baryon fraction measurements, and hence cosmological studies.

Recently, progress has been made in the study the baryon content of the outer regions of clusters through the X-ray observations together with the Sunyaev-Zel'dovich (SZ) effect on the CMB by the hot electrons in the ICM out to $\sim R_{200}$ (Afshordi et al. 2007). A $3 \sigma$ result from the WMAP three-year data suggests that $35 \% \pm 8 \%$ of the thermal energy in ICM is missing, indicating that the baryons in clusters may be missing even accounting for those locked in stars. The result is also supported by independent measurements from other X-ray and SZ observations (Ettori 2003; LaRoque et al. 2006; Vikhlinin et al. 2006; Evrard et al. 2008). Using the X-ray observations together with numerical simulations, Evrard et al. (2008) reported that as much as $50 \%$ of the thermal energy can be missing in the ICM. Although Giodini 
et al. (2009) reported that the total baryon fraction within a smaller radius of $R_{500}$ of massive clusters are consistent to the cosmic value within $1 \sigma$ when all the X-ray hot gas, stellar mass in galaxies, gas depletion during cluster formation, and intracluster light from stars are taken into account, if the missing baryons measured in the outer region $\left(\lesssim R_{200}\right)$ is really significant, this may indicate either a yet-unknown baryonic component, or some new astrophysical processes in the ICM which is driving out the gas from the clusters. While there is no evidence for any undetected baryonic component, Afshordi et al. (2007) pointed out that the missing of hot baryons can either be explained by the thermal diffusion or the evaporation of baryons out of the virial radius of clusters. Another possibility is that electron temperature is lower than that of the equipartition value (Wong et al. 2008).

Given the advancements in the X-ray and SZ observations of the cluster outer regions (Afshordi et al. 2007; George et al. 2009; Reiprich et al. 2009; Bautz et al. 2010), as well as the growing evidence of missing thermal energy in the ICM and the possible negative implications for cosmological tests, a more detailed study of the kinetic processes in cluster envelopes is necessary. While magnetic fields may affect some of the kinetic effects of transport processes such as thermal conduction, the magnetic effects on non-equipartition should not be important since the physics is local. Moreover, it is known that various astrophysical shocks in magnetized environment lead to non-equipartition (Ghavamian et al. 2007; Hull et al. 2001).

The collisionless accretion shock at the outer boundary of a cluster should primarily heat the ions since they carry most of the kinetic energy of the infalling gas. Assuming that cluster accretion shocks are similar to those in supernova remnants, the electron temperature, $T_{e}$, immediately behind the shock would be lower than the ion temperature, $T_{i}$. The equilibration between electrons and ions would then proceed 
by Coulomb collisions. Near the virial radius, due to the low density, the Coulomb collisional timescale can be comparable to the age of the cluster, and the electrons and ions may not achieve equipartition in these regions (Fox \& Loeb 1997). Since X-ray and SZ observations measure the properties of the electrons in the ICM, the net effect is to underestimate the total thermal energy content within clusters. This might account for some or all of the missing thermal energy in the ICM derived by the X-ray and SZ observations. As mentioned above, non-equipartition of ions and electrons is observed in various astrophysical shocks. Most supernova remnants with high Mach numbers comparable to cluster accretion shocks have electron temperatures which are lower than the ion temperatures (Ghavamian et al. 2007); in situ measurements from satellites show the same feature in the Earth's bow shock (Hull et al. 2001). On the other hand, X-ray observations of the merger shock in the Bullet Cluster indicate that the equilibration time may be shorter than that expected from Coulomb collisions alone (Markevitch \& Vikhlinin 2007). However, this merger shock has a Mach number of a few. From both supernova remnant measurements and a physical model, Ghavamian et al. (2007) have shown that electron heating efficiency within a shock front (usually tens of the gyroradius) is inversely proportional to the Mach number squared. If the results can also be applied to the ICM, the low Mach number merger shocks would be immediately heated to $T_{e} / T_{i} \sim 1$, while cosmological accretion shocks with much higher Mach numbers would only be heated to $T_{e} / T_{i} \ll 1$ by collisionless processes. After the electrons and ions pass through the thin shock front, they will likely be equilibrated by Coulomb collisions alone (Bykov et al. 2008a,b).

The non-equipartition in cluster of galaxies has been previously studied by Fox \& Loeb (1997) and Ettori \& Fabian (1998) in semianalytic models. They have shown 
that the temperature difference can be significant in the outer one-third of the shock radius of a cluster. One- or three-dimensional simulations for some individual clusters have also been studied (Chieze et al. 1998; Takizawa 1999; Rudd \& Nagai 2009). While these simulations use different cluster or cosmological models, a general agreement is that the effect of non-equipartition is important if shock heating efficiency of electrons is low $(\ll 1)$ and the equilibration afterward is due to Coulomb collisions alone.

In this chapter, we study systematically the effects of non-equipartition on X-ray and SZ observables in outer regions of relaxed galaxy clusters, which is particularly important for cosmological studies. We carry out one-dimensional hydrodynamic simulations with realistic Navarro-Frenk-White (NFW) model under the concordance $\Lambda \mathrm{CDM}$ cosmological background to provide a sample of clusters (groups) with different masses $\left(10^{13}-10^{16} M_{\odot}\right)$ at different redshifts $(z=0-2)$. Even though we are studying the kinetic non-fluid properties in the cluster outer regions, the hydrodynamic treatment in modeling the cluster dynamical properties is reasonable and is justified as follows. Even dynamically unimportant magnetic fields should be able to reduce significantly the diffusion mean free path perpendicular to the magnetic field (Sarazin 1986; Borgani \& Kravtsov 2009). The suppression of diffusion in a plasma depends on the topology of magnetic fields. For uniform magnetic fields, only diffusion perpendicular to the local magnetic field is suppressed, and along the field, particles move freely; their mean free path along a field line is still determined by Coulomb collisions. On large scales, diffusion is suppressed in bulk only if the magnetic fields are random and highly tangled on small scales. To include anisotropic diffusion in the calculation would be difficult since the magnetic field structure is not known well enough. However, there is some evidence from large-scale magnetohydrodynamic simulations that magnetic fields in galaxy clusters are chaotic with correlation and 
reversal length scales of $\sim 50$ and $\sim 100 \mathrm{kpc}$, respectively (Dolag et al. 2002). Hence, we simply assume that diffusion is suppressed. We also assume that electrons and ions are equilibrated locally on a long Coulomb collisional timescale, and assume that equilibration via plasma instabilities (Schekochihin et al. 2005, 2008) does not occur except at the shocks (see Section 3.3). Previous studies show that the dynamical properties of cluster outer regions in one-dimensional simulations successfully reproduce those simulated in three-dimensional calculations (Navarro et al. 1995; Ryu \& Kang 1997). The advantages of the one-dimensional simulations for our problem are presented in Section 3.2. We emphasize the signatures of non-equipartition on X-ray and SZ observations in our studies. We also study the effect of electron shock heating efficiency on the degree of non-equipartition. Thus, observations of electron-ion equilibration may give constraints to the electron heating efficiency, and hence the electron heating mechanism. The wider parameter space compared to previous work explored in this chapter allows us to study the impact of non-equipartition effects on cosmological studies in Chapter 4.

The chapter is organized as follows. In Section 3.2, we describe the set up of our hydrodynamic models. The detailed implementations of the shock heating and the Coulomb equilibration process for our simulations are presented in Section 3.3. The ability of our simulations to reproduce analytic test models relevant to our studies is discussed in Section 3.4. We present the simulated dynamics of our realistic NFW cluster models in the standard $\Lambda$ CDM cosmology in Section 3.5. These cluster models are used to study the non-equipartition effects presented in the thesis. We define the X-ray and SZ observables for our models to be studied, and also present the results for these observables in Section 3.6. We discuss and conclude our work in Section 3.7. Unless otherwise specified, we assume the Hubble constant $H_{0}=71.9 h_{71.9} \mathrm{~km} \mathrm{~s}^{-1} \mathrm{Mpc}^{-1}$ 
with $h_{71.9}=1$, the total matter density parameter $\Omega_{M, 0}=0.258$, the dark energy density parameter $\Omega_{\Lambda}=0.742$, and the gas fraction $f_{\text {gas }}=\Omega_{b} / \Omega_{M}=0.17$, where $\Omega_{b}$ is the baryon density parameter, for the realistic NFW model in the standard $\Lambda \mathrm{CDM}$ $\operatorname{cosmolog}^{2}$, and a hydrogen mass fraction $X=76 \%$ for the ICM throughout this chapter.

\subsection{Hydrodynamic Model}

LSS cosmological simulations predict that clusters do not evolve in isolation. During the linear phase of structure growth, they can be influenced by tidal forces; while during the nonlinear growth phase, they can grow by accreting a significant number of smaller clusters or merging with clusters with similar sizes. However, here we are interested in studying the structure of accretion shocks in clusters, which can be observed most easily in relaxed clusters which have not undergone a recent major merger. Cosmological studies using the gas fraction in clusters are restricted to clusters with the highest degree of dynamical relaxation to minimize systematic scatter in the determination of cosmological parameters (Allen et al. 2008); thus, relaxed clusters are of particular interest. Moreover, based on a set of high resolution $N$-body simulations, it has been found that the mass accretion history of a dark matter halo in general consists of two distinct phases: an early fast phase and a late slow phase (Wechsler et al. 2002; Zhao et al. 2003a,b; Li et al. 2007). The fast accretion phase is dominated by major mergers, while the slow accretion phase is dominated by smooth accretion of background materials and many minor mergers. We are most interested in studying the non-equipartition of electrons and ions in the outer regions of clusters, where materials should be continuously accreting and the morphology is roughly

\footnotetext{
${ }^{2}$ http://lambda.gsfc.nasa.gov/product/map/dr3/parameters_summary.cfm
} 
spherical symmetric. Therefore, in our models, we simply consider cluster growth by smooth accretion of materials from the background cosmology. In particular, we assume clusters are spherically symmetric and employ one-dimensional hydrodynamic simulations. It has been shown that one-dimensional calculations reproduce the density and temperature profiles of three-dimensional simulations of clusters in the outer regions (Navarro et al. 1995; Ryu \& Kang 1997), where we are most interested. The assumption of spherical symmetry should be sufficient for us to gain insight into the astrophysical effects of non-equipartition on X-ray and SZ observations, as well as the impact on cosmological studies (Wong et al. 2008). Moreover, one-dimensional simulations also allow us to better resolve shocked regions and to isolate the individual physical processes we are interested in, so that non-fluid properties there can be studied in detail. This is difficult to achieve in three-dimensional simulations. Even though three-dimensional simulations have shown that accretion through filaments is a general feature in related clusters, these simulations also show that, other than the filament regions, material is accreted spherically and the morphology in the outskirts of a clusters are roughly spherical symmetric (Molnar et al. 2009). A very recent three-dimensional study has already shown that the signature of non-equipartition for a relaxed cluster is roughly spherical symmetric as well (model CL104 in Rudd \& Nagai 2009).

\subsubsection{Simulation Code}

We employed the PLUTO code (Mignone et al. 2007) to solve the one-dimensional Newtonian hydrodynamic equations in spherical coordinates for our problem. This code provides a multi-physics, multi-algorithm modular environment which allows new physics to be included and new modules to be developed easily. The code does 
not include self-gravity or an $N$-body solver for determining the dark matter distribution, but these can be handled as force terms in the code. We have developed a scheme to include the dark matter contribution to the gravity by evolving the NFW profile self-consistently with the hydrodynamic evolution of the fluid calculated in the code (Section 3.2.5). This implementation has an advantage over using the $N$-body solver, in that the dynamics of gas in the NFW dark matter potential can be investigated under controlled conditions. We have also implemented self-gravity of the gas in the code. Dark energy can also be included in the code easily as a force term (Section 3.2.6). The code is built on modern Godunov-type shock-capturing schemes which are particular suitable for computation of highly supersonic astrophysical flows in the presence of strong discontinuities. Shock-capturing is needed in our calculations since we are interested in calculating the non-equipartition signatures around the shock regions.

\subsubsection{Boundary Conditions and Computational Domain}

The boundary conditions depend on the geometry and the physics of the problem being solved. For a spherically symmetric geometry of the ICM, the inner boundary condition is reflective. Because we are studying the smooth accretion of background materials in isolation, it is natural to use the Hubble-flow-like outer boundary conditions which are defined as

$$
\begin{aligned}
\left.\frac{d v_{g}}{d r}\right|_{\text {in }} & =\left.\frac{d v_{g}}{d r}\right|_{\text {out }}, \\
\rho_{g, \text { in }} & =\rho_{g, \text { out }}
\end{aligned}
$$

and

$$
P_{g, \text { in }}=P_{g, \text { out }}
$$


where $r$ is the radius, $v_{g}, \rho_{g}$, and $P_{g}$ are the gas velocity, mass density, and pressure, respectively, and the subscripts "in" and "out" denote the inner and outer quantities at the outer boundary, respectively. Each simulation is set up such that there is an over density in the central region compared to the critical density (Section 3.2.3). Near the over dense region, materials are accreted toward the center. To avoid boundary effects, the size of the computation domain should be large enough that materials should always be outgoing and follow the Hubble-flow-like outer boundary condition. If the size of the boundary is too small, the dynamics of materials near the boundary would be influenced by the central cluster and would not follow the Hubble-flowlike outflow; in the extreme case, materials should be infalling rather than outgoing. Bertschinger (1985) has shown that in an Einstein-de Sitter universe, materials follow the Hubble-flow-like outflow closely outside of a few times of the turnaround radius (Figure 1(a) therein). The turnaround radius is about $10 \mathrm{Mpc}$ for a cluster with $\sim 10^{15} M_{\odot}$ at present, and hence the estimated radius at where materials follow the Hubble-flow-like outflow is about $30 \mathrm{Mpc}$ from the cluster center. This condition should be sufficient for the $\Lambda \mathrm{CDM}$ universe, since the materials in the $\Lambda \mathrm{CDM}$ universe should be less bound. To be conservative, in our calculations, we take the boundary radius to be at least a few times this estimated radius. In particular, we take the boundary radius to be $100 \mathrm{Mpc}$ in all models. The outer regions in our calculations at the final time reproduce the observed Hubble flow velocity and the baryon density at redshift zero (Figures 3.1 and 3.5 in Sections 3.4 and 3.5, respectively), which validates our choice of the outer boundary conditions.

The volume of each simulation was divided into 1000 spherical annuli. The innermost zone width is set to be $0.42 \mathrm{kpc}$. The widths of the zones increase with radius 
by a fixed ratio $x$,

$$
d r_{i+1}=x d r_{i}
$$

where $d r_{i}$ is the width of the $i$ th zone. The grid is defined such that

$$
\sum_{i=1}^{N} d r_{i}=L
$$

where $N=1000$ is the total number of zones, and $L=100 \mathrm{Mpc}$ is the size of the computational domain. This is called a stretched grid in PLUTO. With this grid of radii, the zones near the accretion shock have widths of less than $1 \%$ of the shock radius in each simulation from $z=0-2$. Doubling the resolution gives essentially the same results.

\subsubsection{Initial Conditions for the Realistic NFW-Dark Energy Cluster Models}

Since at high enough redshift the dynamics of the background universe is close to the Einstein-de Sitter model, the dynamics of a cluster should be close to a self-similar solution as well (Bertschinger 1985). We chose a high initial redshift and set up the initial conditions to be close to the self-similar solution. In particular, the initial condition is such that there are two regions separated by an accretion shock. The location of the cluster accretion shock in the baryonic material is very close to the first caustic in the dark matter, and the distribution and evolution of the baryonic gas and dark matter are almost identical outside the accretion shock (Bertschinger 1985; Ryu \& Kang 1997). Within the shock radius, we assume the dark matter follows the 
NFW profile (Navarro et al. 1995),

$$
\rho_{\mathrm{dm}}(r)=\frac{\rho_{\mathrm{dm}, \mathrm{s}}}{\left(r / r_{s}\right)\left(1+r / r_{s}\right)^{2}}
$$

where $\rho_{\mathrm{dm}, \mathrm{s}}$ is a density scale and $r_{s}$ is the scale radius. The scale radius, $r_{s}$, is related to the concentration parameter, $c$, and the virial radius, $r_{\text {vir }}$, by

$$
r_{s}=r_{\text {vir }} / c
$$

The virial radius, $r_{\text {vir }}$, is defined by

$$
M_{\mathrm{vir}}=\frac{4 \pi}{3} \Delta_{\mathrm{vir}} \rho_{c}(z) r_{\mathrm{vir}}^{3}
$$

where $M_{\text {vir }}$ is the virial mass, $\rho_{c}(z)$ is the critical density of the universe at redshift $z$, and the critical overdensity $\Delta_{\text {vir }}$ is obtained from the solution to the top-hat spherical collapse model, and can be approximated by (Bryan \& Norman 1998)

$$
\Delta_{\mathrm{vir}}=18 \pi^{2}+82 x-39 x^{2}\left(\text { for } \Omega_{R}=0\right),
$$

where $x \equiv\left[\Omega_{M, 0}(1+z)^{3} / E(z)^{2}\right]-1, E(z)^{2} \equiv \Omega_{M, 0}(1+z)^{3}+\Omega_{R}(1+z)^{2}+\Omega_{\Lambda}$, and $\Omega_{R} \equiv 1 /\left(H_{0} R\right)^{2}$ with $R$ here equals the current radius of curvature of the universe. We adopt a concentration parameter given by equation (3.17) in Section 3.2.4, in which $c$ is effectively equal to 4 in all the initial models considered. We also assume that the gas in the initial models is approximately in hydrostatic equilibrium with the dark matter potential. The choice of such initial conditions is convenient because there exists an analytic solution for isothermal hot gas in hydrostatic equilibrium with the dark matter potential (Makino et al. 1998), but not the total potential. This is 
convenient for setting up our initial models. In the simulation runs, the self-gravity of gas is indeed included so that such initial models actually deviate from hydrostatic equilibrium slightly. We have checked that the numerical solutions we are interested in are rather insensitive to the set up of the initial conditions, as long as the initial models are approximately in hydrostatic equilibrium. Beyond the shock radius, both the dark matter and the gas follow the self-similar infalling solutions. The initial time (age of the universe) is chosen to be $29.4 \mathrm{Myr}$, which corresponds to a redshift of $z=70.6$ in the standard $\Lambda$ CDM cosmology. The details of the setup are described below.

We first define an initial cluster mass, $M_{\mathrm{sh}, i}$, at the initial time chosen. The initial cluster mass is distributed within an initial shock radius, $R_{\mathrm{sh}, i}$. In the self-similar solution (Bertschinger 1985), $R_{\mathrm{sh}, i}$ and $M_{\mathrm{sh}, i}$ are related by

$$
R_{\mathrm{sh}, i}=\lambda_{\mathrm{scale}} r_{\mathrm{ta}, i}
$$

and

$$
M_{\mathrm{sh}, i}=\frac{4 \pi}{3} \rho_{c, i} r_{\mathrm{ta}, i}^{3} m_{\mathrm{scale}},
$$

where $r_{\mathrm{ta}, i}$ is the initial turnaround radius, $\rho_{c, i}$ is the critical density at the initial time, and, $\lambda_{\text {scale }}=0.347$ and $m_{\text {scale }}=3.54$ are the dimensionless scaled radius and mass in the self-similar solution at the shock radius, respectively. This implies that the initial average overdensity within the accretion shock is

$$
\Delta_{\text {sh }, i}=m_{\text {scale }} / \lambda_{\text {scale }}^{3}=84.73
$$

Within the shock radius, we assume the dark matter distribution is given by the NFW profile (equation 3.6). For a fixed value of concentration parameter, $c=4$ assumed 
at high redshift (Section 3.2.4), the initial scale radius $r_{s, i}$ can be solved by using equation (C9) in $\mathrm{Hu} \&$ Kravtsov (2003), which is

$$
\frac{r_{s, i}}{R_{\mathrm{sh}, i}}=x\left[f_{\mathrm{sh}, i}=\frac{\Delta_{\mathrm{sh}, i}}{\Delta_{\mathrm{vir}, i}} f(1 / c)\right]
$$

where $f(x)=x^{3}\left[\ln \left(1+x^{-1}\right)-(1+x)^{-1}\right], x(f)$ in an accurate fitting form is given in Appendix C in Hu \& Kravtsov (2003), and $\Delta_{\text {vir }}$ is given in equation (3.9). In general, $c$ depends on mass, and $r_{s}$ can be calculated iteratively. The scale density $\rho_{\mathrm{dm}, \mathrm{s}}$ in equation (3.6) is then fixed by requiring that the total dark matter integrated to the shock radius is equal to $\left(1-f_{\text {gas }}\right) M_{\mathrm{sh}, i}$.

Within the shock radius, we assume the gas is initially in approximately hydrostatic equilibrium $\left(v_{g}=0\right)$ with the dark matter potential. Initially, the gas is assumed to be isothermal. The gas distribution can be solved analytically, and is given by (Makino et al. 1998)

$$
\rho_{g}(r)=\rho_{g, 0} e^{-27 b / 2}\left(1+\frac{r}{r_{s}}\right)^{27 b /\left(2 r / r_{s}\right)} .
$$

The central gas density $\rho_{g, 0}$ and $b$ are fixed by simultaneously requiring that the gas fraction within the shock radius is equal to $f_{\text {gas }}$ and the gas density at the shock radius is given by the strong shock jump condition

$$
\rho_{g 2}=4 \rho_{g 1}
$$

where the subscripts 1 and 2 denote the preshock and postshock quantities, respectively. Once $b$ is fixed, the temperature, and hence the gas pressure is indeed fixed by using equation (9) in Makino et al. (1998). The gas pressure, $P_{g}$, is proportional 
to $\rho_{g}$ since the gas is assumed to be isothermal. However, if such pressure profile is used, the pressure at the shock radius does not match the strong shock jump condition in general. This is because the dynamical solution with accretion shock does not follow the isothermal hydrostatic solution given in Makino et al. (1998). Since we are interested in the shock solution in our calculation, we renormalize the gas pressure such that the strong shock jump condition is satisfied at the shock radius,

$$
P_{g 2}=\frac{4}{3} \rho_{g 1} v_{g 1}^{2}
$$

With this renormalization, the pressure is only lower than the isothermal hydrostatic equilibrium pressure by $17 \%$. The initial gas deviates slight from hydrostatic equilibrium. As long as the gas is in approximately equilibrium initially, the late time evolution of the cluster profiles in the outer regions we are interested in should not be affected by the slightly deviation in the initial condition.

Gas in exact hydrostatic equilibrium with the total gravitational potential satisfying both the density and pressure shock jump conditions is not isothermal in general. We have checked that using initial conditions in exact hydrostatic equilibrium give essentially the same results.

Outside the shock radius, the gas density and velocity profiles are assumed to follow the self-similar infall solution (Bertschinger 1985), and the pressure is set to be effectively zero (i.e., the smallest value the code allows).

\subsubsection{Concentration Parameters}

We also need to set the value of the concentration parameter $c$. Bullock et al. (2001) proposed that $c$ scales as $(1+z)^{-1}$. At low redshift, this is supported by numerical simulations by Wechsler et al. (2002) and Zhao et al. (2003b, 2009), when the dark 
matter halos are in the slow accretion phase. However, at high enough redshift when the dark matter halos are in the fast accretion phase, the concentration parameter approaches a constant minimum value independent of the mass and redshift of the halo (Zhao et al. 2003a, 2009; Gao et al. 2008). At low redshifts, the variation of the concentration parameter with mass can simply be fitted using a power law (Dolag et al. 2004; Neto et al. 2007). Thus, we adopt the concentration parameter used by Buote et al. (2007) but set a minimum value of 4 (Zhao et al. 2009) independent of the redshift and the halo mass of the cluster in our simulations:

$$
c\left(M_{\mathrm{vir}}, z\right)=\max \left[\frac{c_{14}}{1+z}\left(\frac{M_{\mathrm{vir}}}{M_{14}}\right)^{\alpha}, 4\right]
$$

where we take $c_{14}=9.0$ and $\alpha=-0.172$ as determined from the $\mathrm{X}$-ray galaxy clusters with halo masses between $(0.06-20) \times 10^{14} M_{\odot}$ at low redshifts (Buote et al. 2007) and $M_{14} \equiv 10^{14} h_{100}^{-1} M_{\odot}$.

\subsubsection{Self-consistent Evolution of NFW Potential Including Accreted Mass}

At each time step, we determine the gas shock radius, and we assume dark matter is distributed according to the NFW profile within the shock radius; outside that radius, the dynamics of dark matter and gas are the same. We assume the baryon fraction is conserved as materials accrete within the shock, and hence, the dark matter mass within the shock radius is equal to $\left(1 / f_{\text {gas }}-1\right)$ times the gas mass within that region. Although three-dimensional simulations suggest that there is a hydrodynamic outflow of gas and $f_{\text {gas }}$ inside a cluster is smaller than the cosmological value, our treatment here is at least self-consistent. Moreover, a smaller value of $f_{\text {gas }}$ inside a cluster in 
reality would enhance the effect of non-equipartition, and hence our calculations give a conservative estimate of the non-equipartition effect. As mentioned in Section 3.2.3, if the shock radius and the mass within it are known, the dark matter profile can be solved iteratively together with the concentration parameter. The dark matter profile within the shock radius calculated in each time step is used to calculate the gravity contributed by the dark matter within the shock radius.

\subsubsection{Dark Energy Implementation}

We will do the calculations in a cosmological background including the accelerated expansion due to dark energy. The accretion of materials depends sensitively on dark energy since it governs the expansion of the background materials which are to be accreted (Ryu \& Kang 1997). To implement dark energy in the one-dimensional Newtonian code, we utilize the similarity between the Friedmann solution and Newtonian cosmology, since the latter can be solved in the Newtonian code. The dynamical solution of the scale factor, $a$, of a homogeneous universe is governed by

$$
\ddot{a}=-\frac{4 \pi G}{3} a\left(\rho+\frac{3 P}{c^{2}}\right),
$$

where $\rho$ and $P$ are the total energy density and pressure of the universe, respectively, and $G$ and $c$ are the gravitational constant and speed of light, respectively. The Newtonian analog is simply replacing $a$ by $r$, i.e.

$$
\frac{d^{2} r}{d t^{2}}=-\frac{4 \pi G}{3} r\left(\rho+\frac{3 P}{c^{2}}\right)
$$


For a pure dark energy universe where the dark energy is described by the cosmological constant $\Lambda, \rho=\rho_{\Lambda}, P=P_{\Lambda}$, and $\rho_{\Lambda}=-P_{\Lambda} / c^{2}$. Thus, equation (3.19) becomes

$$
\frac{d^{2} r}{d t^{2}}=\frac{8 \pi G}{3} \rho_{\Lambda} r
$$

We identify the right-hand side of equation (3.20) to be the repulsive force per unit mass contributed by the dark energy. The dark energy density, $\rho_{\Lambda}$, in the $\Lambda$ CDM cosmology is constant throughout the history of the universe, and is given by

$$
\rho_{\Lambda}=\Omega_{\Lambda, 0} \rho_{c, 0}
$$

where the quantities on the right-hand side are evaluated at the present time. With the force term in equation (3.20) implemented into the code, the baryon density of the background cosmology is reproduced correctly in each of our simulations (see Figure 3.5 below).

\subsection{Electron Heating within a Cluster}

\subsubsection{General Picture of Shock Heating and the Equilibra- tion between Electrons and Ions Afterward}

The heating of electrons passing through a shock within a cluster involves at least a two-step process. The first step is the shock heating of electrons within the very narrow shock, and the second step is the equilibration between electrons and ions afterward. In this section, we outline the general picture of electron heating within a cluster we assumed for our model. The detailed implementations of the shock heating (first step) and the Coulomb equilibration afterward (second step) are presented in 
Sections 3.3.2 and 3.3.3, respectively. Section 3.3.4 outlines the steps to calculate electron heating in our code.

Immediately after the infalling material (electrons and ions, together with dark matter) has passed through a collisionless accretion shock, we assume it should primarily heat the ions rather than electrons since they carry most of the kinetic energy of the infalling gas. The electron temperature immediately behind the shock would be lower than the ion temperature, and they would be in a state of non-equipartition. Indeed, non-equipartition of ions and electrons is known in various astrophysical shocks. Most supernova remnants with high Mach numbers comparable to cluster accretion shocks have electron temperatures which are lower than the ion temperatures (Ghavamian et al. 2007); in situ measurements from satellites show the same feature in the Earth's bow shock (Hull et al. 2001). It is reasonable to assume that cluster accretion shocks are similar to those astrophysical shocks. Within the very narrow shock region, electrons and ions can exchange energy by collisionless processes generated by plasma instabilities there. Unfortunately, the collisionless heating mechanisms and the rate of heating are still poorly known theoretically. We simply model the shock heating efficiency for the electron as a free parameter (Section 3.3.2).

After the electrons and ions have passed through the thin shock front, they will then be equilibrated by Coulomb collisions (Bykov et al. 2008a,b). It is also possible that behind the shock, electrons and ions can exchange energy by collisionless processes generated by plasma instabilities left behind. Again, the collisionless heating mechanisms and the rate of equilibration are still poorly known theoretically. It has been argued that behind a strong shock, the spectra of supernova remnants are consistent with purely Coulomb equilibration processes (Rakowski et al. 2003). Given both the theoretical uncertainty concerning the collisionless heating mechanisms and 
at least some observational evidence that collisionless processes are not important in the equilibration downstream, we simply consider the Coulomb collisions as the only equilibration process behind the shock in our calculations (Section 3.3.3).

In calculating the equilibration processes of electrons and ions, we consider a fully ionized plasma of hydrogen and helium only. The timescale for charged particles of the same species to achieve a Maxwellian distribution can be estimated by the collisional timescale (Spitzer 1962, p. 133)

$$
t_{x x}=28.6 \mathrm{Myr}\left(\frac{T_{x}}{10^{7} \mathrm{~K}}\right)^{3 / 2}\left(\frac{n_{x}}{10^{-5} \mathrm{~cm}^{-3}}\right)^{-1}\left(\frac{\ln \Lambda}{40}\right)^{-1} \frac{A_{x}^{1 / 2}}{Z_{x}^{4}},
$$

where $T_{x}$ is the temperature of the species $x$ after it has achieved equilibrium, $n_{x}$ is the number density, $A_{x}$ is the particle mass number, $Z_{x}$ is the particle charge, and $\ln \Lambda$ is the Coulomb logarithm which is similar for all species of interest. For the charged particles we are interested in, the electron-electron collisional timescale is the shortest, with $t_{e e} \sim t_{p p} A_{e}^{1 / 2} \sim t_{p p} / 43$. For typical abundance $n_{\mathrm{H}} \sim 10 n_{\mathrm{He}}, t_{p p} \sim t_{\mathrm{He}^{+2} \mathrm{He}^{+2}}$. For protons, the collisional timescale is $t_{p p} \sim 30 \mathrm{Myr}$, which is much shorter than the age of the cluster or the accretion timescale. For an accretion shock propagating outward with a velocity $v_{s} \sim 1000 \mathrm{~km} \mathrm{~s}^{-1}$, protons at a distance $d \sim v_{s} t_{p p} / 4 \sim 7 \mathrm{kpc}$ away from the shock should be in a Maxwellian distribution. This is of the order of the zone size $(\sim 10 \mathrm{kpc})$ at the shock radius in our calculations. Hence, we can assume that protons, helium ions, and electrons are in equilibrium independently with temperatures $T_{p}, T_{\mathrm{He}^{+2}}$, and $T_{e}$, respectively. The energy exchange timescale for two species of particles, $x$ and $y$ in Maxwellian distributions with temperatures $T_{x}$ and $T_{y}$ 
is given by the equilibration timescale (Spitzer 1962, p. 135)

$$
t_{x y}=14.7 \operatorname{Myr}\left(\frac{T_{x}}{10^{7} \mathrm{~K}}\right)^{3 / 2}\left(\frac{n_{y}}{10^{-5} \mathrm{~cm}^{-3}}\right)^{-1}\left(\frac{\ln \Lambda}{40}\right)^{-1} \frac{A_{y}}{Z_{x}^{2} Z_{y}^{2} A_{x}^{1 / 2}}\left(1+\frac{T_{y}}{T_{x}} \frac{A_{x}}{A_{y}}\right)^{3 / 2}
$$

Consider energy exchange between electrons and protons, and assume that the electrons are initially much cooler than the protons. When $T_{e} \lesssim T_{p}\left(m_{e} / m_{p}\right)$, the electrons are heated rapidly, with $t_{e p} \sim t_{p p} / 3600$. Thus, we expect that most of the time spent in electron heating will occur in the regime in which $T_{e} / T_{p} \gg m_{e} / m_{p}$. Hence, the energy exchange timescale between electrons and protons, $t_{e p}$ is on the order of $0.5\left(m_{p} / m_{e}\right)^{1 / 2} t_{p p} \sim 21 t_{p p}$. Similarly, $t_{e \mathrm{He}^{+2}} \gtrsim t_{e p}$ for $n_{\mathrm{H}} \sim 10 n_{\mathrm{He}}$. The equipartition timescale between electrons and ions (either protons or helium ions) will be of the order of $0.6 \mathrm{Gyr}$, which is comparable to the accretion timescale of a cluster, [(cluster size)/(accretion velocity)] $\sim 1$ Gyr. Thus, $T_{e}$ may not be equal to $T_{p}$ or $T_{\mathrm{He}^{+2}}$ in the outer regions of a cluster, which is the problem we are considering. From equation (3.23), it can be seen that $t_{\mathrm{He}^{+2} p} \gtrsim t_{p p}$. It is also possible that $T_{p} \neq T_{\mathrm{He}^{+2}}$ in the regions of interest. The solutions of the equilibration of the plasma formally can be obtained by solving the equilibration between the three species. If we are interested only in the electron temperature evolution, we can consider the energy exchange rate between the electrons and both of the two ion species

$$
1 / t_{e i}=1 / t_{e p}+1 / t_{e \mathrm{He}^{+2}}
$$

Since the contribution per ion to $t_{e i}^{-1}$ is the same for protons and helium ions (equation 3.23), the rate of change of electron temperature with the plasma, $d T_{e} / d t$, depends only on the mean ion temperature (Fox \& Loeb 1997). Moreover, once the ions reach equilibrium with each other, the rate of change of their temperatures due 
to collisions with electrons is the same, so they will remain in equilibrium. Thus, for simplicity, we assume a single ion temperature, $T_{i}$, and we assume the electrons with temperature $T_{e}$ are equilibrated with this single ion temperature plasma.

In hydrodynamic simulations, the three hydrodynamic variables, the gas mass density, $\rho_{g}$, the total gas pressure, $P_{g}$, and the gas velocity, $v_{g}$, determine the hydrodynamic state of the fluid completely, independent of the kinetic state of the plasma. Since we are interested in the difference between electron temperature, $T_{e}$, and ion temperature, $T_{i}$, we assign a new variable, $\tau \equiv T_{e} / \bar{T}$, in each grid of our hydrodynamic calculations, where $\bar{T}$ is the average thermodynamic temperature of the fluid given by $\bar{T} \equiv\left(\mu m_{p} / k_{B}\right)\left(P_{g} / \rho_{g}\right)$, where $\mu=0.59$ is the mean molecular weight, $m_{p}$ is the proton mass, and $k_{B}$ is the Boltzmann constant. The electron temperature, $T_{e}$, the ion temperature, $T_{i}$, and the average thermodynamic temperature, $\bar{T}$, are simply related by

$$
\bar{T}=\frac{n_{e} T_{e}+n_{i} T_{i}}{n_{e}+n_{i}}
$$

where $n_{e}$ and $n_{i}$ are the electron and ion number densities, respectively.

\subsubsection{Electron Heating within the Thin Shock Front}

Within the very narrow shock front, electrons can be heated by both adiabatic compression and non-adiabatic heating. Non-adiabatic heating includes the conversion of the bulk kinetic energy into thermal energy and other collisionless heating processes generated by plasma instabilities. We can define the total non-adiabatic electron heating efficiency in the shock, $\beta$, to be the change in electron temperature due to non-adiabatic heating, $\Delta T_{e, \text { non-ad }}$, relative to the change in average thermodynamic 
temperature due to non-adiabatic heating, $\Delta \bar{T}_{\text {non-ad }}$,

$$
\beta \equiv\left(\frac{\Delta T_{e}}{\Delta \bar{T}}\right)_{\text {non-ad }}
$$

The change in the bulk kinetic energy per particle is $m\left(v_{g 1}^{2}-v_{g 2}^{2}\right) / 2$, where $m$ is the particle mass, and hence the increase in the electron temperature is much smaller than that of the ions in a shock. For the electron heating processes generated by plasma instabilities, the details of the mechanism and the efficiency are still unclear. Therefore, in our study, we assume total non-adiabatic electron heating efficiency, $\beta$, to be a constant for any given cluster model. Adiabatic compression increases the electron temperature by a factor of $\left(\rho_{e 2} / \rho_{e 1}\right)^{\gamma-1}$, where $\gamma$ is the adiabatic index, $\rho_{e 1}$ and $\rho_{e 2}$ are the preshock and postshock electron temperatures, respectively. For a strong shock with $\gamma=5 / 3$, adiabatic compression only increases the electron temperature by a factor of $2^{4 / 3} \approx 2.5$. Thus, if the preshock electron temperature, $T_{e 1}$, is negligible compared to the postshock average temperature, $\bar{T}_{2}$, adiabatic heating would not be important. This is indeed the case in our models. Nevertheless, we have included adiabatic heating in our calculations. Including both adiabatic and non-adiabatic heating, the postshock electron temperature immediately after the shock is given by (Appendix A in this chapter)

$$
T_{e 2}=\left(\frac{\rho_{g 2}}{\rho_{g 1}}\right)^{\gamma-1} T_{e 1}+\beta \max \left[0, \bar{T}_{2}-\left(\frac{\rho_{g 2}}{\rho_{g 1}}\right)^{\gamma-1} \bar{T}_{1}\right]
$$

where a minimum of zero in the second term is set to ensure that numerical fluctuations do not introduce a false decrease in entropy in the non-adiabatic heating.

Note that we assume that the heating of electrons is equivalent to the increase of temperature. This is only true if the electron distribution is Maxwellian. The time 
for the electrons to achieve a Maxwellian distribution after they have been heated at the shock is $t_{e e} \sim t_{p p} / 43 \sim 0.7 \mathrm{Myr}$ (Section 3.3.1). As shown in Section 3.3.1, this is much shorter than the accretion timescale, and the electrons within the zone size at the shock radius of our simulations should achieve the Maxwellian distribution. Note also that even if electrons are in non-Maxwellian distribution and if such electrons are heated much faster than the Coulomb collisional heating rate, the faster heating within the shock has already been parameterized by the electron heating efficiency $\beta$.

Recently, observations of supernova remnants have shown that the electron heating efficiency at the shock is inversely proportional to the Mach number squared (Ghavamian et al. 2007). If the result can be applied to cluster accretion shocks, the high accretion shock Mach number $(\mathcal{M}>100)$ would imply the electron heating efficiency to be $\ll 1$ in cluster accretion shocks. In our work, we consider two cases for the shock heating of electrons: $\beta=1 / 1800$ (the mass ratio of electrons to protons) as a model for a very low non-adiabatic heating efficiency which is supported by supernova remnant observations, and $\beta=0.5$ as a model for an intermediate heating efficiency within the shock.

\subsubsection{Coulomb Equilibration After Shock Heating}

After the shock-heated material has passed through the thin shock front, we assume Coulomb equilibration as the only heating process for electrons behind the shock. The evolution of electron temperature due to Coulomb collision is given by (Spitzer 1962, p. 135)

$$
\frac{d \tau}{d t}=\frac{2 \ln \Lambda}{503}\left\langle\frac{Z^{2}}{A}\right\rangle \frac{n}{\bar{T}^{3 / 2}} \tau^{-3 / 2}(1-\tau) \mathrm{s}^{-1},
$$

where $n$ is the total particle number density, $\ln \Lambda \approx 37.8+\ln \left(T_{e} / 10^{8} \mathrm{~K}\right)-$ $\ln \left(n_{e} / 10^{-3} \mathrm{~cm}^{-3}\right)^{1 / 2}$ is the Coulomb logarithm, the angle bracket term is the mean 
value over the ratio of the square of ion charge $Z$ and the atomic number $A$. For our model of a pure hydrogen and helium gas, the angle bracket term equals 1 as long as $T_{e} / T_{i} \gtrsim m_{e} / m_{p}$, which is true for our models with a minimum $\beta=1 / 1800$. We solve equation (3.28) for each time step in our hydrodynamic calculations (Section 3.3.4).

\subsubsection{Steps to Calculate the $T_{e} / \bar{T}$ Evolution}

Cosmological accretion shocks are identified as shocks in which the Mach number, $\mathcal{M}$, is greater than 10 . In our simulations, we use the pressure shock jump condition,

$$
\frac{P_{g 2}}{P_{g 1}}=\frac{2 \gamma}{\gamma+1} \mathcal{M}^{2}-\frac{\gamma-1}{\gamma+1}
$$

to identify shocks. In each time step of our hydrodynamic simulations, if the condition of $\mathcal{M}>10$ in equation (3.29) is satisfied, we identify there is a strong shock at $r_{2}$. We found that this condition can identify cosmological shocks correctly in our simulations. In regions without a strong shock, we calculate the $T_{e} / \bar{T}$ evolution using equation (3.28) throughout the entire time step. If an accretion shock is identified, we first use equation (3.28) to evolve $T_{e} / \bar{T}$ for one half of the time step. Then, we apply the shock heating using equation (3.27). Finally, we use equation (3.28) again to evolve $T_{e} / \bar{T}$ for the reminding half of the time step.

Cosmological simulations show that there are various kinds of shocks formed in the LSSs (Kang et al. 2007). Based on their location, these shocks can be classified as external and internal shocks. External shocks are formed around the outermost surfaces of the LSSs that unshocked intergalactic gas is falling onto (sheets, filaments, and halos), and the gas is shock heated for the first time. These external shocks in general have Mach numbers $\gg 10$. Internal shocks have low Mach numbers $\lesssim 10$. They are formed within those nonlinear structures, e.g., galaxy clusters and filaments, 
by the infall of previously shocked gas during subclump mergers, as well as by chaotic flow motions. These cosmological simulations show that there are a large number of low Mach number internal shocks contributing significantly to the thermal energy budget of a cluster (Kang et al. 2007). Since those small Mach number internal shocks are mainly found well within inner regions of a cluster where the densities are high enough, electrons and ions are in general in equipartition. Here, we are only interested in the non-equipartition effects of electrons and ions, and these effects are only significant in very low density regions (e.g., cosmological accretion shock regions) rather than the contribution of the thermal energy from the shocks to the cluster. For our one-dimensional simulations which are used to study relaxed clusters, due to the symmetry of the problem, there is in fact only one cosmological accretion shock with Mach number $\gg 10$ for each cluster, and there are no internal shocks formed. For comparison, three-dimensional simulations have shown that for relaxed clusters, the number of small Mach number internal shocks is much smaller than that of the high Mach number external shocks (Molnar et al. 2009). This is because internal shocks are formed mostly by mergers or chaotic flow motions which are less likely to be found in relaxed clusters by definition. Thus, we do not have to consider electron heating by the low Mach number internal shocks at least for relaxed cluster that we are interested in. Future three-dimensional simulations will be needed to test our assumption.

\subsection{Test Models: Self-similar Models}

The essential physics governing the equilibration problem we are considering are the hydrodynamics of the gas, the gravity by both dark matter and gas, the equilibration physics between electrons and ions, as well as the dark energy which modifies the 
background cosmology, and hence the rate of accretion onto clusters and the duration of the accretion throughout cosmic history. The contribution of dark energy can be tested by whether the baryon density of the background cosmology can be reproduced correctly in each of our simulations, and this is indeed the case for our realistic simulations which will be presented in Section 3.5 (see Figure 3.5 below). We present

three tests here to show that our hydro code can handle the necessary physics correctly for the problem we are considering. The hydrodynamical response of the gas in an external gravitational field is tested by reproducing the analytic self-similar solutions of a collisionless dark matter dominated accretion model (Bertschinger 1985). The correct handling of self-gravity of the gas is tested by reproducing the analytic selfsimilar solutions of a collisional gas dominated accretion model (Bertschinger 1985). Finally, the correct handling of the equilibration physics is tested by comparing to the analytic solutions of a self-similar non-equipartition model calculated by Fox \& Loeb (1997).

\subsubsection{Collisionless Dark Matter Dominated Accretion Model}

In this section, we compare our numerical simulation with the analytic self-similar solution of the collisionless dark matter dominated accretion model in the Einstein-de Sitter universe $\left(\Omega_{M}=1, \Omega_{\Lambda}=0\right)$. In this model, we assume gravity is dominated by the dark matter potential, and hence the self-gravity of the gas is switched off in the code. This is done to test the ability of our code to handle an external gravitational potential, which is important for our realistic simulations with the NFW dark matter potential included. We set $\Omega_{b}$ to be 0.05 in this model. We assume the current Hubble constant to be $H_{0}=71.9 \mathrm{~km} \mathrm{~s}^{-1} \mathrm{Mpc}^{-1}$ in this simulation run, and hence at redshift $z=0$, the critical density is $\rho_{c}(z=0)=9.71 \times 10^{-30} \mathrm{~g} \mathrm{~cm}^{-3}$. The 
initial gas profiles are set according to the self-similar solution given in Bertschinger (1985). The gravitational potential due to the dark matter is calculated according to the self-similar solution at each time step. The gas evolution within the dark matter gravitational potential is then calculated by the PLUTO code. Figure 3.1 shows the dynamical variables of our numerical simulations for the self-similar model at four different redshifts. The model shown in the three panels is such that the dark matter mass accreted within $R_{178}$ is $M_{178}=10^{15} M_{\odot}$ at $z=0$. The figure shows that a strong shock is propagating from $R_{\mathrm{sh}} \approx 0.7 \mathrm{Mpc}$ at $z=2$ to $R_{\mathrm{sh}} \approx 3 \mathrm{Mpc}$ at $z=0$. The shock velocity decreases from $v_{\mathrm{sh}} \approx 1500 \mathrm{~km} \mathrm{~s}^{-1}$ at $z=2$ down to $v_{\mathrm{sh}} \approx 1300 \mathrm{~km} \mathrm{~s}^{-1}$ at $z=0$. At $z=0$, the gas density just within the shock is about $0.8 \rho_{c}(z=0)$, and drops to about $0.2 \rho_{c}(z=0)$ just beyond the shock, while a very sharp jump in pressure can be seen at the shock radius. At about $100 \mathrm{Mpc}$, which is at the edge of our simulation domain, the density drops according to the background cosmology as $\Omega_{b} \rho_{c}$ (inset in the middle panel of Figure 3.1). The velocity profile at very large radii also follows the Hubble flow (inset in the top panel of Figure 3.1).

To compare with the analytic self-similar solution directly, we scale the physical radius and the dynamical variables to obtain the scaled dimensionless valuables according to the scaling relations given in Bertschinger (1985),

$$
\begin{aligned}
\lambda & =r / r_{\mathrm{ta}}, \\
v(\lambda) & =\frac{v_{g}(r, t)}{r_{\mathrm{ta}} / t}, \\
\phi(\lambda) & =\frac{\rho_{g}(r, t)}{\rho_{c} \Omega_{b}}, \\
\psi(\lambda) & =\frac{P_{g}(r, t)}{\rho_{c} \Omega_{b} r_{\mathrm{ta}}^{2} / t^{2}},
\end{aligned}
$$

where $\lambda, v, \phi$, and $\psi$ are the dimensionless radius, velocity, density, and pressure 
of the gas, respectively, $t$ is the cosmic time which is equal to $1 / \sqrt{6 \pi G \rho_{c}}$ for the Einstein-de Sitter universe. The scaled dimensionless valuables in our simulation are shown as solid lines in Figure 3.2. The analytic solutions are plotted as open circles for comparison. After scaling, the dimensionless valuables in our simulation at the four different redshifts, as well as the analytic solutions, all lie almost along the same locus which can hardly be distinguished on the figures. This shows that our numerical simulations are in excellent agreement with the analytic solutions within all region of our interest $(\sim 0.1-10 \mathrm{Mpc})$. We conclude here that our code can calculate the dynamics of the gas, as well as handling the shock in an external gravity under the cosmological expansion correctly.

\subsubsection{Collisional Gas Dominated Accretion Model}

Since we have included self-gravity of the gas in our realistic simulations, it is necessary to check whether our code can handle self-gravity correctly. We have performed a set of simulations to compare with the analytic self-similar solution of the collisional gas accretion model in the Einstein-de Sitter universe $\left(\Omega_{M}=\Omega_{b}=1, \Omega_{\Lambda}=0\right)$. In this model, the universe is considered as purely collisional fluid, and hence we do not include the dark matter contribution in these simulations. Self-gravity of the gas is included in the simulations. We scaled the physical radius and the dynamical variables according to equation (3.30) with $\Omega_{b}=1$. The scaled dimensionless valuables in our simulations are shown as the solid lines of Figure 3.3. The analytic solutions are plotted as open circles for comparison. Again, the figure shows that our numerical simulations are in excellent agreement with the analytic solutions. We conclude here that our code can calculate the dynamics of the gas, as well as handle the shock with self-gravity under the cosmological expansion correctly. 


\subsubsection{Self-similar Non-equipartition Model}

With the self-similar dynamical cluster model calculated in Section 3.4.1 from our simulations, we can calculate the degree of non-equilibration of the gas following the method given in Section 3.3. In Figure 3.4, the ratios of the electron and average thermodynamic temperature, $\tau$, for two clusters with different masses are shown as circles. The corresponding lines are the analytic solutions (Fox \& Loeb 1997). Our simulations are in very good agreement with the analytic solutions. The slightly deviations are mainly due to the finite resolution of the shock region in our numerical simulations. We conclude here that our code can calculate the equilibration physics correctly.

\subsection{Dynamics for Realistic NFW-Dark Energy Models}

Table 3.1 lists the masses and radii for different overdensities for some representative NFW cluster models in the standard $\Lambda$ CDM cosmology at $z=0$. The different definitions of masses and radii are used interchangeably throughout this chapter. In Figure 3.5, we show the evolution of the dynamical variables $\left(v_{g}, \rho_{g}\right.$, and $\left.P_{g}\right)$ as a function of radius for a cluster with an accreted mass of $M_{\mathrm{vir}}=1.19 \times 10^{15} M_{\odot}$ at $z=0$, where $M_{\text {vir }}=M_{95}$ in the $\Lambda$ CDM cosmology we assumed. The total mass within the shock radius is $M_{\mathrm{sh}}=1.53 \times 10^{15} M_{\odot}$ at $z=0$. The figure shows that a strong shock propagates from $R_{\mathrm{sh}} \approx 1.2 \mathrm{Mpc}$ at $z=2$ to $R_{\mathrm{sh}} \approx 4.2 \mathrm{Mpc}$ at $z=0$. The shock velocity decreases from $v_{\mathrm{sh}} \approx 1400 \mathrm{~km} \mathrm{~s}^{-1}$ at $z=2$ down to $v_{\mathrm{sh}} \approx 1000 \mathrm{~km} \mathrm{~s}^{-1}$ at $z=0$. Similarly to the self-similar dark matter dominated model, at $z=0$, the gas density just within the shock is about $0.8 \rho_{c}(z=0)$, and drops to about $0.2 \rho_{c}(z=0)$ 
just beyond the shock. At about $100 \mathrm{Mpc}$ which is at the edge of our simulation domain, the density drops according to the background cosmology as $\Omega_{b} \rho_{c}$ (inset in the middle panel of Figure 3.5). The pressure profile flattens as the NFW cluster evolves, and the central pressure drops. This is in contrast to the self-similar dark matter dominated model where the pressure never drops even in the very central region $<0.1 \mathrm{Mpc}$ (not shown on the graph). The velocity profile at large radius also follows the Hubble flow correctly (the inset of Figure 3.5). This shows that with our implementation of the dark energy, our simulations can reproduce the background cosmology correctly. For comparison, the shock radius and the shock velocity for a self-similar dark matter dominated cluster with the same mass $\left(M_{\mathrm{sh}}\right)$ in the Einstein-

de Sitter universe $\left(\Omega_{M}=1, \Omega_{\Lambda}=0\right)$ are $R_{\text {sh }}^{\mathrm{SS}}=3.11 h_{71.9}^{-2 / 3}\left(M_{\mathrm{sh}} / 1.53 \times 10^{15} M_{\odot}\right)^{1 / 3} \mathrm{Mpc}$ and $v_{\mathrm{sh}}^{\mathrm{SS}}=1.38 \times 10^{3} h_{71.9}^{1 / 3}\left(M_{\mathrm{sh}} / 1.53 \times 10^{15} M_{\odot}\right)^{1 / 3} \mathrm{~km} \mathrm{~s}^{-1}$, respectively. For the realistic NFW-DE model, the shock radius is larger than that of the self-similar solution by a factor of 1.38, while the shock velocity is lower by a factor of 1.35 . The result is consistent with the one-dimensional $N$-body simulation given by Ryu \& Kang (1997). Their results show that the shock radius and the shock velocity for a cluster in the $\Lambda \mathrm{CDM}$ cosmology with $\Omega_{\Lambda} \approx 0.74$ is about 1.4 larger and 1.35 lower than those in the Einstein-de Sitter universe, respectively.

\subsection{Observables for Realistic NFW-Dark Energy Models}

\subsubsection{Definition of X-ray Observables}

X-ray spectra depend mainly on electron temperature rather than ion temperature, and the projected temperature profile of a cluster can be directly measured from 
X-ray observations. Different weighting schemes have been used to calculate the projected temperature from hydrodynamic simulations, and Mazzotta et al. (2004) have shown that most of the commonly used weighting schemes (the mass-weighted and the emission-weighted) give significantly different results from the X-ray observed spectroscopic temperature, $T_{\mathrm{spec}}$, and the spectroscopic-like temperature calculated from an analytic weighting scheme they developed is able to approximate $T_{\text {spec }}$ to better than a few percent for temperature above $\sim 3 \mathrm{keV}$. However, for the non-equipartition model we considered, the electron temperature in the outer regions can be as low as $0.1 \mathrm{keV}$. X-ray line emission from heavy elements contribute significantly to the Xray spectrum for temperature below $\sim 3 \mathrm{keV}$. Such line emission is not considered by Mazzotta et al. (2004). Vikhlinin (2006) has generalized the spectroscopic-like temperature to lower temperatures down to $\sim 0.5 \mathrm{keV}$ and to arbitrary metallicity. This generalized scheme takes into account both the continuum and line emission assuming the MEKAL emission model (Mewe et al. 1985; Kaastra \& Mewe 1993; Liedahl et al. 1995). The detector response has also been taken into account. The weighting scheme is no longer analytic and has to be tabulated. Following the algorithm of Vikhlinin (2006), we calculate the projected spectroscopic-like temperature, $T_{\mathrm{sl}}$, from our numerical simulations. The integration is carried out within the shock radius. The latest Chandra ACIS-S aim point response files released for the ACIS Cycle 11 proposal planning are used to generate the weighting table ${ }^{3}$. Using different Chandra CCD response files does not affect the results significantly (Vikhlinin 2006).

We also calculate the surface brightness profile from our numerical simulations. Here, we define $x$ as the projected radial distance from a cluster center. The surface

\footnotetext{
${ }^{3}$ http://cxc.harvard.edu/caldb/prop_plan/imaging/index.html
} 
brightness profile in a given energy band $E$ is given by

$$
S_{E}(x)=\int \Lambda_{E}\left(T_{e}, Z\right) n_{e} n_{p} d l
$$

where $\Lambda_{E}\left(T_{e}, Z\right)$ is the cooling function which depends only on the electron temperature and heavy element abundances, $Z, n_{p}$ is the proton number density, and $l$ is the distance along the line of sight. The integration is carried out within the shock radius. The cooling function $\Lambda_{E}\left(T_{e}, Z\right)$ is calculated using the MEKAL model (Mewe et al. 1985; Kaastra \& Mewe 1993; Liedahl et al. 1995).

For our models with non-equipartition considered, $T_{e}$ is used to calculate the $T_{\text {sl,non-eq }}$ and $S_{E \text {,non-eq }}$. For comparison, we also consider models with electrons and ions are fully in equipartition by taking $T_{e}=\bar{T}$, which is usually assumed in the literature. Quantities calculated with full equipartition assumed are denoted with the subscript "eq".

Note that in the outer region where electrons and ions may not be in equipartition, non-equilibrium ionization may also be important. This may increase line emissions in the soft bands $(E \lesssim 1 \mathrm{keV})$. In our calculations, non-equilibrium ionization is not considered.

\subsubsection{Definition of SZ Observables}

The SZ effect by a cluster at $x$ can be characterized as a temperature increment, $\Delta T_{\mathrm{SZE}}(x)$, with respect to the $\mathrm{CMB}$ spectrum

$$
\Delta T_{\mathrm{SZE}}(x)=f(\theta) y(x) T_{\mathrm{CMB}}
$$


where $\theta=h \nu / k_{B} T_{\mathrm{CMB}}$ is the dimensionless frequency, $T_{\mathrm{CMB}}$ is the CMB temperature, $y$ is the Comptonization parameter, and $f(\theta)$ is given by

$$
f(\theta)=\left(\theta \frac{e^{\theta}+1}{e^{\theta}-1}-4\right)\left[1+\delta_{\mathrm{SZE}}\left(\theta, T_{e}\right)\right]
$$

For simplicity, we neglect the relativistic term, $\delta_{\mathrm{SZE}}$, which is not important for frequency lower than about $250 \mathrm{GHz}$. The Comptonization parameter is given by

$$
y=\frac{k_{B} \sigma_{\mathrm{T}}}{m_{e} c^{2}} \int n_{e} T_{e} d l \propto \int P_{e} d l,
$$

where $\sigma_{\mathrm{T}}$ is the Thomson scattering cross section and $P_{e}=n_{e} k_{B} T_{e}$ is the electron pressure.

Similarly to the calculation for the spectroscopic-like temperature, for our models in non-equipartition and in fully equipartition, we calculate the $\Delta T_{\text {non-eq/eq }}(x)$ by integrating equation (3.34) along the line of sight within the shock radius.

Another useful SZ observable is the integrated Comptonization parameter, $Y$, which is defined as the integration of the Comptonization parameter in equation (3.34) on the sky

$$
Y=d_{A}^{2} \int y d \Omega=\int y d A
$$

where $d_{A}$ is the angular diameter distance to the cluster, $\Omega$ is the solid angle of the cluster on the sky, and $A$ is the projected surface area. We integrate the projected surface area of the cluster up to the shock radius. Such a quantity is useful for spatially unresolved clusters with SZ observations where the solid angle covers the whole cluster.

Since $Y=\int y d A \propto \int P_{e} d V \propto \int n_{e} T_{e} d V$, where $V$ is the volume of the cluster, the integrated Comptonization parameter $Y$ is basically measuring the thermal energy 
of the electrons in the cluster. If electrons and ions are not in equipartition and if the electron temperature is lower than that of the ions globally, the value of $Y$ measured would be lower than the equipartition value. To characterize the degree

of non-equipartition of the whole cluster, we define the bias as the ratio $Y_{\text {non-eq }} / Y_{\text {eq }}$, where $Y_{\text {non-eq }}$ is the integrated Comptonization parameter for the non-equilibration model and $Y_{\text {eq }}$ is that for the equipartition model $\left(T_{e}=\bar{T}\right)$.

It has been shown that the integrated Comptonization parameter displays a tight correlation with cluster mass (Reid \& Spergel 2006). Such a tight correction is needed for precision cosmology, and hence a correct understanding of the integrated Comptonization parameter is important. A detailed discussion of the use of SZ surveys to study cosmology can be found in Carlstrom et al. (2002).

\subsubsection{Results for the Temperature Profiles and the X-ray Observables}

The evolution of the average thermodynamic temperature as a function of radius for a cluster with an accreted mass of $M_{\mathrm{sh}}=1.53 \times 10^{15} M_{\odot}$ at $z=0$ in the $\Lambda \mathrm{CDM}$ cosmology is shown in Figure 3.6. In general, for each redshift, the temperature profile rises from the very central region to a peak, and then drops toward the outer region. The drop of temperature in the outer region is due to the drop of the shock velocity during the accretion history. The central drops in temperature, as well as the decrease of the value of the peak temperature are due to the adiabatic expansion of the cluster during the evolution. This is in contrast to the average thermodynamic temperature of the self-similar solution in the Einstein-de Sitter universe where the temperature profile is always rising toward the central region at all redshift (Figure 3.7), and the temperature within the same gas mass, $M_{\text {gas }}$, never drops during the accretion 
history. The expansion of the hot gas in the central region of the cluster in our simulation is probably caused by the evolution of the NFW profile, since there is a central pressure drop for the gas in the NFW potential but not for the case in the self-similar model (Section 3.5). In a real cluster, the temperature profile of the central region $(\lesssim 100 \mathrm{kpc})$ is likely to be complicated by physical processes such as cooling, AGN heating (Fabian et al. 2000; Blanton et al. 2001), and perhaps thermal conduction (Narayan \& Medvedev 2001; Chandran \& Maron 2004; Lazarian 2006). On another hand, in the outer region of a cluster, the thermodynamic state is likely to be dominated by gravitational processes and shock heating. Our models have included the essential physics in the outer regions.

Figure 3.8 shows the ratio of electron and average thermodynamic temperatures, $\tau \equiv T_{e} / \bar{T}$, as a function of radius for the same cluster at $z=0$. The shock radius, $R_{\mathrm{sh}} \approx 4.2 \mathrm{Mpc}$, is about 1.5 times the virial radius, $R_{\mathrm{vir}} \approx 2.8 \mathrm{Mpc}$. Within the virial radius, the temperature differences between electrons and ions are less than $1 \%$, while beyond $R_{\text {vir }}, T_{e} / \bar{T}$ decreases from $\sim 1$ to $1 / 1800$ at the shock radius for the model with $\beta=1 / 1800$. For the $\beta=0.5$ model, $T_{e} / \bar{T}$ decreases from $\sim 1$ to 0.5 at the shock radius. In general, $T_{e} / \bar{T}$ decreases from $\sim 1$ to $\beta$ at the shock radius for our models. Our models predict that $T_{e} / \bar{T} \sim 0.8$ at $r \approx 0.9 R_{\mathrm{sh}} \approx 3.8 \mathrm{Mpc}$ for a range of $\beta$ between $1 / 1800$ and 0.5 . Beyond that radius, $T_{e} / \bar{T}$ depends rather strongly on $\beta$. The strong dependence at the shock radius can be used to distinguish shock heating models or constraint the shock heating efficiency of electrons at the shock.

The solid line in Figure 3.9 shows the projected spectroscopic-like temperature profiles, $T_{\text {sl,non-eq }}$, of the $M_{\mathrm{sh}}=1.53 \times 10^{15} M_{\odot}$ cluster near the outer region at $z=0$ for our non-equipartition model. We assume $\beta=1 / 1800$ and $Z=0.3 Z_{\odot}$ in this figure. For comparison, we also plot the projected spectroscopic-like temperature for 
a model where equipartition of electrons and ions throughout the cluster is assumed $\left(T_{e}=T_{i}=\bar{T}\right)$ as a dashed line. Both projected temperatures drop in the outer region as the radius increases, but the electron temperature in the non-equipartition model drops faster in most regions shown. Note that for the equipartition model, the projected temperature drop at the very last data point is a numerical artifact instead of a real feature. This is due to the finite resolution of the shock handling in the hydro code which causes a slightly lower temperature compared to the idealized solution. Such an artifact does not affect our results significantly.

The ratio of the projected temperature profiles of the two models, $\tau_{\text {proj }} \equiv$ $T_{\text {sl,non-eq }} / T_{\mathrm{sl} \text {,eq }}$, is plotted in Figure 3.10. Models for $\beta=0.5$ and $Z=0.1 Z_{\odot}$ are also plotted for comparison. Compared to the deprojected (or physical) temperature ratio (Figure 3.8), the deviation is larger for the projected temperature profiles, which is directly determined observationally. This is because for the projected temperature profile, electrons in the outer region also contribute to the inner region. For models with $\beta=1 / 1800$ and $Z=0.3 Z_{\odot}$, there is a $\sim 10 \%$ difference in the projected temperatures for the equipartition and the non-equipartition models at the virial radius ( $R_{\mathrm{vir}} \approx 2.8 \mathrm{Mpc}$ ) in contrast to less than a percent for the deprojected temperatures. The projected temperature difference increases to about $20 \%$ at a radius of $\sim 3.3 \mathrm{Mpc}$, which is about 1.2 of the virial radius. Even for $\beta=0.5$, the difference at this radius can be as large as $\sim 15 \%$. We also found that the non-equipartition effect on the projected temperature profiles is enhanced by metallicity. From $Z=0.1$ to $0.3 Z_{\odot}$, the deviation at a radius of $\sim 2.8(3.3) \mathrm{Mpc}$ is enhanced by a factor of $\sim 1.7(1.5)$ for the $\beta=1 / 1800$ model. This is because the domination of the line emissions in the soft band spectra is enhanced by increasing metallicity, which is more important for the non-equipartition model where electron temperature is lower. 
The effect of non-equipartition is larger for more massive clusters. This is shown in Figure 3.11, where $\tau_{\text {proj }}$ for clusters with different masses are plotted versus $r / R_{\mathrm{sh}}$. This is because the deviation of the physical temperatures $T_{e}$ and $\bar{T}$ increases with cluster mass. The behavior qualitatively agrees with the analytic self-similar solution in the Einstein-de Sitter universe found by Fox \& Loeb (1997).

The surface brightness profiles for various energy bands of the $M_{\mathrm{sh}}=1.53 \times 10^{15} M_{\odot}$ cluster near the outer region at $z=0$ are shown in Figure 3.12. The ratios $S_{\text {non-eq }} / S_{\text {eq }}$ are plotted in Figure 3.13. We assume $\beta=1 / 1800$ and $Z=0.3 Z_{\odot}$ in these figures. For X-ray emission below $\sim 2 \mathrm{keV}$, the relative difference between the nonequipartition and the equipartition models is smaller than that of the projected temperature profile. This is because the surface brightness depends on density squared but has a weaker dependence on temperature. The relative difference for X-ray emission above $\sim 2 \mathrm{keV}$ is similar to that of the projected temperature profile because of the energy cut off for lower temperature. The differences in surface brightness in all energy bands shown are $\lesssim 10 \%$ for radii $\lesssim 3$ Mpc. Beyond $\sim 3$ Mpc where the electron temperature drops significantly below $\sim 3 \mathrm{keV}$ for the non-equipartition model, the differences in surface brightness profiles become important. The difference in Xray surface brightness is most significant for the hard band (2.0-10.0 keV) compared to the soft $(0.3-1.0 \mathrm{keV})$ and medium $(1.0-2.0 \mathrm{keV})$ bands. For the soft band, the surface brightness for the non-equipartition model actually becomes larger than that of the equipartition model at large radii. This is because of the increase in the soft band emissivity for temperature below $\sim 1 \mathrm{keV}$. We have also plotted the full X-ray band $(0.3-10.0 \mathrm{keV})$ and the bolometric surface brightness profiles in Figure 3.12. The full X-ray band surface brightness profile for the non-equipartition model is always lower than that of the equipartition model, while the opposite is true for the 
bolometric surface brightness profile. This indicates that for the non-equipartition model, a large amount of emission occurs in the energy band below $0.3 \mathrm{keV}$ compared to that of the equipartition model. In fact, the bolometric surface brightness near the shock radius for the non-equipartition model can reach $\sim 35$ times that of the equipartition model.

Current X-ray observations by Suzaku of cluster outer regions extend to only $R_{200} \sim 2 \mathrm{Mpc}$ where the non-equipartition effects on both the surface brightness and projected temperature profiles are $\lesssim 1 \%$ in our models. The sensitivities of these observations are limited by the Poisson variations in the background extragalactic source density rather than by the instrumental background (Bautz et al. 2010). To push the sensitivity limit out to $\sim R_{\text {vir }} \approx 1.4 R_{200}$ where non-equipartition effects on the projected temperature are $\sim 10 \%$, observations combining high spatial resolution and high surface brightness sensitivity would be needed. On the other hand, increasing the coverage of solid angle can also help to reduce the Poisson variations. Here, we estimate how much improvement in sensitivity would be needed to push the current limit of $\sim R_{200}$ out to $R_{\mathrm{vir}} \approx 1.4 R_{200}$. Assume most of the current observations only have sensitivities out to $R_{200}$ (but see George et al. 2009) and cluster surface brightness scales as shown in Figure 3.12. From Figure 3.12, $S\left(R_{200}\right) \sim 6 S\left(1.4 R_{200}\right)$ for the $0.3-10.0 \mathrm{keV}$ band, and hence to have a significant detection of X-ray emission at $R_{\mathrm{vir}} \approx 1.4 R_{200}$, a factor of $\sim 6$ improvement in sensitivity would be needed. Probably a combination of the above two solutions (a factor of 6 improvement in each case) is needed to push the sensitivity limit to $\sim 1.4 R_{200}$. Pushing the limit to $R_{\text {sh }}$ will be very challenging with X-ray observations. Recently, George et al. (2009) reported that cluster emission has been detected out to $\sim 1.5 R_{200}$ in the cluster PKS0745-191. They have shown that the temperature at that radius is $\sim 30 \%$ lower than the tem- 
perature predicted by hydrodynamic simulations. If their results are confirmed, we suggest that this may be a signature of electron-ion non-equipartition.

\subsubsection{Results for the SZ Temperature Decrement}

Compared to X-ray observations, it is believed that cluster outer regions should be better studied by future SZ observations because the SZ effect depends only on the electron density to the first power, while X-ray emission depends on the electron density squared. The X-ray signature drops much faster compared to the SZ signature. Moreover, the SZ effect is independent of redshift, and a large number of high-redshift clusters should be observable. It has been suggested that future SZ observations such as the Atacama Large Millimeter Array (ALMA) should be able to detect or rule out the presence of accretion shocks in clusters (Kocsis et al. 2005; Molnar et al. 2009).

Figure 3.14 shows the evolution of the temperature decrement magnitude due to the $\mathrm{SZ}$ effect, $-\Delta T_{\mathrm{SZE}}$, as a function of radius for a cluster with mass accreted to $M_{\mathrm{sh}}=1.53 \times 10^{15} M_{\odot}$ at $z=0$. The solid line shows the non-equipartition model and the dotted line shows the equipartition model. Both the equipartition and nonequipartition models show very similar SZ temperature decrement profiles, with the SZ temperature decrement dropping faster in the non-equipartition models. For the cluster at $z=0,-\Delta T_{\mathrm{SZE}}$ drops from $\sim 1 \mathrm{mK}$ at about $0.1 \mathrm{Mpc}$ down to $\sim 1 \mu \mathrm{K}$ at about $3.5 \mathrm{Mpc}$, and then drops very rapidly beyond that. For the same cluster at $z=2$ when it had a mass of $M_{\mathrm{sh}}(z=2)=6.72 \times 10^{14} M_{\odot}$ (corresponding to $M_{\mathrm{vir}}=M_{169}=5.30 \times 10^{14} M_{\odot}$ at that redshift), the sharp drop in $-\Delta T_{\mathrm{SZE}}$ occurs from a higher value of about $10 \mu \mathrm{K}$ near the shock radius. This suggests that the shock feature can be best studied through high-redshift clusters provided that the region of interest can be spatially resolved. To show the signature of non-equipartition effect 
more clearly, we plot the difference between the SZ temperature decrements for the equipartition and the non-equipartition models, $\delta \Delta T_{\mathrm{SZE}}=\Delta T_{\mathrm{SZE}, \text { non-eq }}-\Delta T_{\mathrm{SZE}, \text { eq }}$, in Figure 3.15. It shows that the difference is larger at higher redshift for a given cluster. At $z=2, \delta \Delta T_{\mathrm{SZE}}$ is of the order of $1 \mu \mathrm{K}$ for the $M_{\mathrm{sh}}(z=2)=6.72 \times 10^{14} M_{\odot}$ cluster we considered. The ratios of the temperature decrements (or equivalently the Comptonization parameters), $y_{\text {non-eq }} / y_{\text {eq }}=\Delta T_{\mathrm{SZE} \text {,non-eq }} / \Delta T_{\mathrm{SZE}, \text { eq }}$, are also plotted in Figure 3.16. Similarly to the temperature profiles, for $z=0$ at the virial radius of $R_{\mathrm{vir}} \approx 2.8 \mathrm{Mpc}, y_{\text {non-eq }} / y_{\text {eq }}$ is about 0.93 , while it drops to about 0.8 at a radius of $\sim 3.5 \mathrm{Mpc}$.

The detailed analysis of whether the effect can be distinguished observationally involves a discussion of the detail characteristic of the potential radio observations, which will be given in Appendix A at the end of this thesis ${ }^{4}$. Here, we only estimate roughly the possibility of whether the non-equipartition signature can be detected at the shock region. We closely follow the estimation done by Kocsis et al. (2005) and Molnar et al. (2009). In their work, they studied whether two different models with and without a shock can be distinguished in future ALMA observations from the SZ effect. In principle, we can adopt their technique directly to distinguish our two models with clusters in equipartition or not. In Figure 10(c) of Molnar et al. (2009), they have argued that for two cluster models with $\delta \Delta T_{\mathrm{SZE}}$ of the order of a few $\mu \mathrm{K}$, the signal-to-noise ratio $(\mathrm{S} / \mathrm{N})$ for distinguishing between their models can be as high as 70 . For our model with the $M_{\mathrm{sh}}(z=0)=1.53 \times 10^{15} M_{\odot}$ cluster, $\delta \Delta T_{\mathrm{SZE}}$ is less than $\sim 2 \mu \mathrm{K}$ even at $z=2$. However, the most massive cluster today can reach a mass as high as $M_{200}(z=0) \approx 2 \times 10^{15} M_{\odot}$. In Figure 3.17, we show the $-\Delta T_{\mathrm{SZE}}$ profiles for the equipartition and the non-equipartition models of a cluster

\footnotetext{
${ }^{4}$ We will show that it will be very difficult to distinguish the non-equipartition model and equipartition model in a single observation of a cluster with ALMA at the end of this thesis.
} 
with $M_{200}(z=0)=1.83 \times 10^{15} M_{\odot}$, which corresponds to $M_{\mathrm{sh}}(z=0)=3.06 \times 10^{15} M_{\odot}$. The profiles are similar to those of the $M_{\mathrm{sh}}(z=0)=1.53 \times 10^{15} M_{\odot}$ cluster, except the magnitudes are larger. The shock radius at $z=2$ is about $1.5 \mathrm{Mpc}$, which corresponds to an angular size of $177^{\prime \prime}$ for the $\Lambda \mathrm{CDM}$ cosmology we assumed. The mass within the shock radius at $z=2$ is equal to $M_{\mathrm{sh}}(z=2)=1.34 \times 10^{15} M_{\odot}$. Figure 3.18 shows the deviation in $\Delta T_{\mathrm{SZE}}$ between the equipartition and non-equipartition models. At $z=2$, the deviation is $\delta \Delta T_{\mathrm{SZE}} \approx 4-5 \mu \mathrm{K}$ near the shock radius. Closely following Kocsis et al. (2005) and Molnar et al. (2009), we estimate the S/N for distinguishing between the equipartition and the non-equipartition models of the very massive cluster at $z=2$ by ALMA. Kocsis et al. (2005) estimated that a $\sim 20 \mathrm{hr}$ on-source integration time for ALMA would be enough to achieve a sensitivity of $\lesssim 10 \mu \mathrm{K}$, but the latest ALMA Sensitivity Calculator ${ }^{5}$ shows that about $260 \mathrm{hr}$ would be needed for the required angular resolution (FWHM) of $2^{\prime \prime}$ at $100 \mathrm{GHz}$ with 64 antennas. Though such a very long observation is possible, we lower the required angular resolution (FWHM) to $4^{\prime \prime}$ to increase the sensitivity, and the required on-source integration time is reduced to $\sim 17 \mathrm{hr}$. Hence we assume a $\sim 17 \mathrm{hr}$ on-source integration time and estimate the $\mathrm{S} / \mathrm{N}$ ratio to be $\approx N_{\text {pix }}^{1 / 2}(\mathrm{~S} / \mathrm{N})_{1}$, where $N_{\text {pix }}$ is the number of independent pixels in the region of interest, and $(\mathrm{S} / \mathrm{N})_{1}$ is the $\mathrm{S} / \mathrm{N}$ for a single pixel in ALMA. From Figure 3.18, the width of the annular region of interest from $\sim 1$ to $\sim 1.5 \mathrm{Mpc}$ is about $0.5 \mathrm{Mpc} \sim 59^{\prime \prime}$ at $z=2$, where $1 \mathrm{Mpc}$ corresponds to $118^{\prime \prime}$ at this redshift for the standard $\Lambda$ CDM universe assumed. Assuming a Gaussian beam with an angular diameter (FWHM) of $4^{\prime \prime}$ for ALMA, the number of beams within the region of interest are about $N_{\text {pix }} \approx 3000$. In a real cluster, the accretion would likely not to be spherical, and material would accrete through filaments. However, in regions other than the filaments, accretion shocks are roughly spherical and those are the

\footnotetext{
${ }^{5}$ http://www.eso.org/sci/facilities/alma/observing/tools/etc/index.html
} 
regions which our model may be applied. Molnar et al. (2009) have estimated the area coverage factor of such spherical accretion shock region to be $\sim 50 \%$. With this correction, $N_{\mathrm{pix}, 50 \%} \approx 1500$. The noise for a single pixel is estimated to be $10 \mu \mathrm{K}$ (Kocsis et al. 2005; Molnar et al. 2009). We take the signal for a single pixel to be $S_{1} \approx \delta \Delta T_{\mathrm{SZE}} \approx 4 \mu \mathrm{K}$ (Figure 3.18). Thus, we obtain an $\mathrm{S} / \mathrm{N}$ of $\sim 16$. Even if the area coverage factor goes down to only $10 \%$, the $\mathrm{S} / \mathrm{N}$ can still be as high as 7. As noted in Molnar et al. (2009), the sensitivity of interferometers is reduced for large-scale smooth surface density distributions, which is perhaps the case for the SZ effect in the cluster outer regions. The $\mathrm{S} / \mathrm{N}$ in this analysis may be overestimated. However, they also argue that such a reduction in sensitivity can be recovered by using nonlinear de-convolution of data from mosaic observations (Helfer et al. 2002; Molnar et al. 2009). The above estimation assumes that the cluster model parameters

(e.g., cluster mass, shock radius) are known in advance. In real observations trying to distinguish between equipartition and non-equipartition models, if those parameters cannot be obtained by other means, we may need to fit the parameters from the data. This will in general reduce the $\mathrm{S} / \mathrm{N}$ estimated above. We defer a more detailed study to Appendix A at the end of this thesis.

\subsubsection{Results for the Integrated SZ Biases, $Y_{\text {non-eq }} / Y_{\text {eq }}$, and its Evolution}

Figure 3.19 shows the integrated SZ biases, $Y_{\text {non-eq }} / Y_{\text {eq }}$ defined in Section 3.6.2 as a function of $M_{\mathrm{sh}}$ for both our simulated realistic NFW model in the $\Lambda$ CDM universe and the numerical simulated self-similar model in the Einstein-de Sitter universe at different redshifts. We assume $f_{\text {gas }}=0.17$ for models in the Einstein-de Sitter universe in Figure 3.19. The masses are evaluated at the labeled redshift so that 
the evolutionary history for a particular cluster cannot be seen on the graph. Both models show that $Y_{\text {non-eq }} / Y_{\text {eq }}$ increases as the cluster mass increases, which is expected as the effect of non-equipartition increases with mass. For the realistic NFW model, $Y_{\text {non-eq }} / Y_{\text {eq }}$ decreases from 1 for $M_{\text {sh }}=10^{13} M_{\odot}$ down to $\sim 0.9$ for $M_{\text {sh }}=10^{16} M_{\odot}$ at $z=0$. The upper mass limit to which a cluster can grow is limited by the background cosmology as well as the initial density fluctuation amplitudes. The most massive nearby cluster observed has a mass of about $M_{200} \approx 2 \times 10^{15} M_{\odot}$, which corresponds to $M_{\mathrm{sh}} \approx 3.3 \times 10^{15} M_{\odot}$ in our NFW model. $Y_{\text {non-eq }} / Y_{\text {eq }}$ for the most massive cluster in our universe is hence about 0.97 if it is nearby $(z \approx 0)$. This bias would be larger ( $Y_{\text {non-eq }} / Y_{\text {eq }}$ smaller in magnitude) at higher redshift for a given mass.

Recent observations suggest that a significant fraction $(20 \%-40 \%)$ of the thermal energy is missing from clusters (Ettori 2003; LaRoque et al. 2006; Vikhlinin et al. 2006; Afshordi et al. 2007; Evrard et al. 2008, but see also Giodini et al. 2009). Obviously, if electrons and ions are in non-equipartition, the thermal energy measured by X-ray or SZ observations should be reduced. Our simulations suggest that for cluster with $M_{\mathrm{sh}} \sim 1.5 \times 10^{15} M_{\odot}\left(M_{200} \sim 10^{15} M_{\odot}\right)$, the non-equipartition effect can account for only about $2 \%-3 \%$ of the missing thermal energy. For the most massive clusters, up to $3 \%-4 \%$ of the thermal energy beyond the equipartition value may be stored in the ions near the shock radius, if electrons and ions are in non-equipartition. For $M_{\mathrm{sh}}$ smaller than about $5 \times 10^{14} M_{\odot}$ at $z=0-2$, the non-equipartition effect is less than $1 \%$.

For the self-similar model, the $Y_{\text {non-eq }} / Y_{\text {eq }}$ curves at the four different redshifts actually lie almost along the same line which cannot be easily distinguished from the graph (Figure 3.19). This shows that the integrated SZ bias for the self-similar model does not evolve with redshift. In contrast, the integrated SZ bias for the realistic 
NFW model in the $\Lambda$ CDM universe evolves with redshift. In the $\Lambda$ CDM universe, the expansion of the universe starts to accelerate around the redshift where $\Omega_{M} \sim \Omega_{\Lambda}$; this breaks the self-similar solution for cosmological accretion, and hence we should expect $Y_{\text {non-eq }} / Y_{\text {eq }}$ would also deviate from self-similarity in general. For our realistic NFW model in the $\Lambda$ CDM universe, the integrated SZ bias decreases as $z$ decreases. This is probably due to the decreasing rate of accretion onto clusters in the $\Lambda$ CDM universe during the cosmological acceleration, which results in a relatively longer timescale for the electron-ion equilibration inside a cluster compared to a cluster with the same mass in the Einstein-de Sitter universe. At $z=0, Y_{\text {non-eq }} / Y_{\text {eq }}$ of the realistic NFW model is smaller than that of the self-similar model with the same $f_{\text {gas }}$ for $M_{\mathrm{sh}}>10^{15} M_{\odot}$, but the effect is similar for $M_{\mathrm{sh}}<10^{15} M_{\odot}$ for both models. At higher redshifts, the effect of non-equipartition is larger for the realistic NFW model for the entire mass range.

Though the magnitude of $Y_{\text {non-eq }} / Y_{\text {eq }}$ is small for the range of cluster masses, even a percentage level deviation in the most massive clusters is important for precision cosmology studies. Cosmological studies using the mass function evolution depend sensitively on the high mass end of clusters. For cluster mass count surveys using $Y$ as a mass proxy, if the bias in $Y$ is not properly taken into account, the resulted mass function would be biased low at the high mass end (i.e., less massive clusters would be observed if clusters are in non-equipartition). Even though the mass- $Y$ relation can be self-calibrated, the evolution of $Y_{\text {non-eq }} / Y_{\text {eq }}$ may introduce a bias if the self-calibration is not properly done at each redshift. For example, if the mass and $Y$ relation is selfcalibrated correctly for low-redshift clusters but this calibration is extrapolated to high-redshift clusters, bias would be introduced if the non-equipartition effect is not properly taken into account. A detailed study of the effect of non-equipartition on SZ 
surveys and the implication to cosmological studies will be presented in Chapter 4 .

\subsection{Discussions and Conclusions}

Using one-dimensional hydrodynamic simulations, we have calculated a sample of realistic NFW clusters in a range of masses in the $\Lambda$ CDM cosmology. The cluster properties we simulated are consistent with the one-dimensional $N$-body simulations by Ryu \& Kang (1997), and they have shown that their calculations reproduce the density and temperature profiles of the three-dimensional simulated relaxed clusters in the outer regions. Our one-dimensional hydrodynamic simulations help us to isolate the important physical processes under controlled conditions. We have studied in detail the effect of non-equipartition in the outer regions of relaxed clusters in the $\Lambda \mathrm{CDM}$ cosmology.

Using $f_{\text {gas }}=0.17$ (which is the upper limit for a cluster), we give a conservative lower limit of the non-equipartition effect on clusters. We have shown that for a cluster with a mass of $M_{\mathrm{sh}} \sim 1.5 \times 10^{15} M_{\odot}$, within $R_{\mathrm{vir}}$, electron and ion temperatures only differ by less than a percent. Our results show that the effect is smaller than those calculated from recent three-dimensional simulations, which shows that $T_{e}$ can be biased low by $5 \%$ at $R_{200} \sim 0.7 R_{\text {vir }}$ (model CL104 in Rudd \& Nagai 2009). A detailed analysis is needed to address the difference, but a possible explanation may be that in a three-dimensional cluster the accretion shock can be formed further in. Our results show that $T_{e} / \bar{T}$ can reach $\approx 0.8$ for a range of non-adiabatic electron heating efficiency $\beta \sim 1 / 1800$ to 0.5 at $\sim 0.9 R_{\text {sh }}$ (or $\sim 1.4 R_{\text {vir }}$ ). Beyond that radius, $T_{e} / \bar{T}$ depends rather strongly on $\beta$, and such a strong dependence at the shock radius can be used to distinguish shock heating models or constrain the shock heating efficiency of electrons. We also show that the effect of non-equipartition is larger for more 
massive clusters, which is consistent to analytic self-similar models in the Einstein-de Sitter universe (Fox \& Loeb 1997).

Using the algorithm developed by Vikhlinin (2006) which takes into account the soft emission at low temperature down to $\sim 0.5 \mathrm{keV}$, arbitrary metallicity, and instrumentation response, we calculated the X-ray spectroscopic-like temperature profiles which are the one to be directly determined observationally. The effect of nonequipartition on the projected temperature profiles is larger than that on the deprojected (or physical) temperature profiles. Non-equipartition effects can introduce $\mathrm{a} \sim 10 \%$ bias in the projected temperature at $R_{\text {vir }}$ for a wide range of $\beta$. This is because for the projected temperature profile, electrons in the outer region also contribute to the inner region. We also found that the effect of non-equipartition on the projected temperature profiles can be enhanced by increasing metallicity. This is because the domination of the line emissions in the soft band spectra is enhanced by increased metallicity, which is more important for the non-equipartition model where the electron temperature is lower.

The effect of non-equipartition on X-ray surface brightness profile in the $0.3-$ $2 \mathrm{keV}$ band is smaller than that on the projected temperature profile. This is because the surface brightness depends on density squared but with a weaker dependence on temperature. This means that in the outer regions, clusters in non-equipartition have similar X-ray surface brightness profile for $E \lesssim 2 \mathrm{keV}$, but with bigger difference in temperature compared to those equipartition counterparts. For $E \gtrsim 2 \mathrm{keV}$, non-equipartition effects on X-ray surface brightness profiles are similar to those on the projected temperature profiles. For a cluster with $M_{\text {sh }} \sim 1.5 \times 10^{15} M_{\odot}$, the effect of non-equipartition on surface brightness profiles in all energy bands is $\lesssim 10 \%$ for radii $\lesssim 3 \mathrm{Mpc}$; beyond that, the effect can be important. We found that for 
the non-equipartition model, the surface brightness profile in the low-energy band $\lesssim 1 \mathrm{keV}$ can be higher than that of the equipartition model in the cluster outer regions. Non-equilibrium ionization, which was not considered in our calculations of the emissivities, may further enhance the line emissions in the soft bands $(E \lesssim 1 \mathrm{keV})$.

Current X-ray observations extend to only $\sim R_{200} \sim 2 \mathrm{Mpc}$, although some results from recent Suzaku observations begin to go a bit beyond that (George et al. 2009). Within those regions with $\lesssim R_{200}$, electrons and ions should be almost in equipartition and the signatures in the X-ray temperature and surface brightness should be rather weak. But future X-ray observations may extend to $\sim R_{\text {vir }} \approx 1.4 R_{200}$ or even close to the shock radius. We have shown that non-equipartition of electrons and ions should be detectable in those studies. The results by George et al. (2009) support our conclusion.

The effects of non-equipartition on the SZ effect were studied. At $z=0$, the effect on the Comptonization parameters is similar to that of the projected temperature profiles. For a cluster with $M_{\mathrm{sh}} \sim 1.5 \times 10^{15} M_{\odot}, y_{\text {non-eq }} / y_{\text {eq }} \approx 0.93(0.8)$ at $1(1.3) R_{\text {vir }}$. For a given cluster, the difference between the SZ temperature decrements for the equipartition and the non-equipartition models is larger at a higher redshift. For the most massive clusters at $z \approx 2$, the differences can be $\delta \Delta T_{\mathrm{SZE}} \approx 4-5 \mu \mathrm{K}$ near the shock radius. A detailed analysis of whether the equipartition and non-equipartition models near the shock region can be distinguished by, for example, ALMA, will be presented in Appendix A at this end of this thesis.

The effects on the integrated SZ Comptonization parameter, which measures the thermal energy content of the electrons, were studied. We have shown that the integrated SZ bias, $Y_{\text {non-eq }} / Y_{\text {eq }}$, increases as the cluster mass increases, which is expected as the effect of non-equipartition increases with mass. In general, the non- 
equipartition effect is larger for the realistic NFW model in the $\Lambda$ CDM universe than that for the self-similar model in the Einstein-de Sitter universe, assuming that they have the same $f_{\text {gas }}$. Our simulations suggest that for relaxed clusters with $M_{\mathrm{sh}} \sim 1.5 \times 10^{15} M_{\odot}$, the non-equipartition effect can account for only about $2 \%-3 \%$ of the missing thermal energy globally. For the most massive clusters, up to $4 \%-5 \%$ of the thermal energy beyond the equipartition value may be stored in the thermal energy of ions near the shock radius, but for clusters with $M_{\mathrm{sh}} \lesssim 5 \times 10^{14} M_{\odot}$, the non-equipartition effect is less than $1 \%$. Thus, we argue that, at least for relaxed clusters, the non-equipartition effect alone can only account for some of the missing thermal energy problem, if any, for high-mass clusters but not for clusters with smaller masses. On the other hand, this suggests that hot gas may be missing due to other astrophysical processes not yet known, and the $f_{\text {gas }}$ in a real cluster should be lower than that we used in our numerical simulations. We have estimated that reducing $f_{\text {gas }}$ by $20 \%$ will enhance the local non-equipartition effect near the outer region by a few percent, but the integrated SZ bias, $Y_{\text {non-eq }} / Y_{\text {eq }}$ is not affected by more than a percent.

We emphasis here that even though non-equipartition effects may not affect the global energy budget significantly, the effect is still important locally in the outer regions $\left(\sim R_{\text {vir }}\right)$ of a cluster. Future X-ray and SZ observations may extend out to $R_{\mathrm{sh}}$, and the effect of non-equipartition should be considered when studying cluster properties in those regions.

We found that for our realistic NFW model in the $\Lambda$ CDM universe, $Y_{\text {non-eq }} / Y_{\text {eq }}$ evolves with redshift, which is in contrast to the self-similar model in the Einsteinde Sitter universe. For our realistic NFW model in the $\Lambda$ CDM universe, $Y_{\text {non-eq }} / Y_{\text {eq }}$ decreases as $z$ decreases. This is probably due to the decreasing rate of accretion onto 
clusters in the $\Lambda \mathrm{CDM}$ universe during the period of cosmological acceleration, which results in a relatively longer timescale for the electron-ion equilibration inside a cluster compared to a cluster with the same mass in the Einstein-de Sitter universe. Though the magnitude of $Y_{\text {non-eq }} / Y_{\text {eq }}$ is small for the range of cluster masses, even a percentage level deviation in the most massive clusters can be important for precision cosmology studies. Such a variation of $Y$ with $z$ would introduce an apparent evolution in $f_{\text {gas }}$, which would bias the cosmological studies using the $f_{\text {gas }}$ techniques (Allen et al. 2008). Recently, Rudd \& Nagai (2009) have shown that the non-equipartition effect on $Y$ can be enhanced by major mergers up to $30 \%$, although for low Mach number mergers, the shock heating efficiency for electrons may be higher which can weaken the non-equipartition effect (Ghavamian et al. 2007; Markevitch \& Vikhlinin 2007). The temporary boost due to mergers may have a significant effect on the estimation of cosmological parameters using clusters. We defer a detailed study of the effect on cosmology studies in Chapter 4. 


\section{Appendix A Expression for Electron Heating within the Shock}

Adiabatic changes in electron temperature are given by

$$
\left(T_{e 2}\right)_{\mathrm{ad}}=\left(\frac{\rho_{e 2}}{\rho_{e 1}}\right)^{\gamma-1} T_{e 1}
$$

where $\rho_{e 2} / \rho_{e 1}=\rho_{g 2} / \rho_{g 1}$ for fully ionized plasma. In addition to the adiabatic heating, electrons can also be heated by non-adiabatic processes. From the definition of non-adiabatic electron heating efficiency (equation (3.26)), the change of electron temperature due to non-adiabatic heating is

$$
\left(\Delta T_{e}\right)_{\text {non-ad }}=\beta(\Delta \bar{T})_{\text {non-ad }}
$$

Thus, the final electron temperature can be expressed as

$$
T_{e 2}=\left(\frac{\rho_{g 2}}{\rho_{g 1}}\right)^{\gamma-1} T_{e 1}+\left(\Delta T_{e}\right)_{\text {non-ad }}
$$

Similarly for the average thermodynamic temperature, we have

$$
\left(\bar{T}_{2}\right)_{\mathrm{ad}}=\left(\frac{\rho_{g 2}}{\rho_{g 1}}\right)^{\gamma-1} \bar{T}_{1}
$$

and

$$
\begin{aligned}
(\Delta \bar{T})_{\text {non-ad }} & =\bar{T}_{2}-\left(\bar{T}_{2}\right)_{\mathrm{ad}} \\
& =\bar{T}_{2}-\left(\frac{\rho_{g 2}}{\rho_{g 1}}\right)^{\gamma-1} \bar{T}_{1}
\end{aligned}
$$


Combining equations (A.2), (A.3), and (A.5), we get

$$
T_{e 2}=\left(\frac{\rho_{g 2}}{\rho_{g 1}}\right)^{\gamma-1} T_{e 1}+\beta \max \left[0, \bar{T}_{2}-\left(\frac{\rho_{g 2}}{\rho_{g 1}}\right)^{\gamma-1} \bar{T}_{1}\right]
$$

where a minimum of zero in the second term is set to ensure that numerical fluctuations do not introduce false decreases in entropy in the non-adiabatic heating. 
Table 3.1. Masses and Radii ${ }^{\mathrm{a}}$ of Some Representative NFW Cluster Models in the Standard $\Lambda$ CDM Cosmology at $z=0$

\begin{tabular}{cccccccc}
\hline \hline$M_{\text {sh }}$ & $R_{\mathrm{sh}}$ & $M_{\mathrm{vir}}^{\mathrm{b}}$ & $R_{\text {vir }}$ & $M_{200}$ & $R_{200}$ & $M_{500}$ & $R_{500}$ \\
\hline 7.65 & 3.31 & 6.05 & 2.19 & 4.90 & 1.60 & 3.59 & 1.06 \\
15.3 & 4.22 & 11.9 & 2.75 & 9.50 & 1.99 & 6.83 & 1.32 \\
30.6 & 5.41 & 23.1 & 3.43 & 18.3 & 2.48 & 12.9 & 1.63 \\
\hline
\end{tabular}

${ }^{\text {a }}$ Masses are in the unit of $10^{14} M_{\odot}$ and radii are in the unit of Mpc.

b $\Delta_{\text {vir }}=95.3$ at $z=0$ for the standard $\Lambda \mathrm{CDM}$ cosmology. 

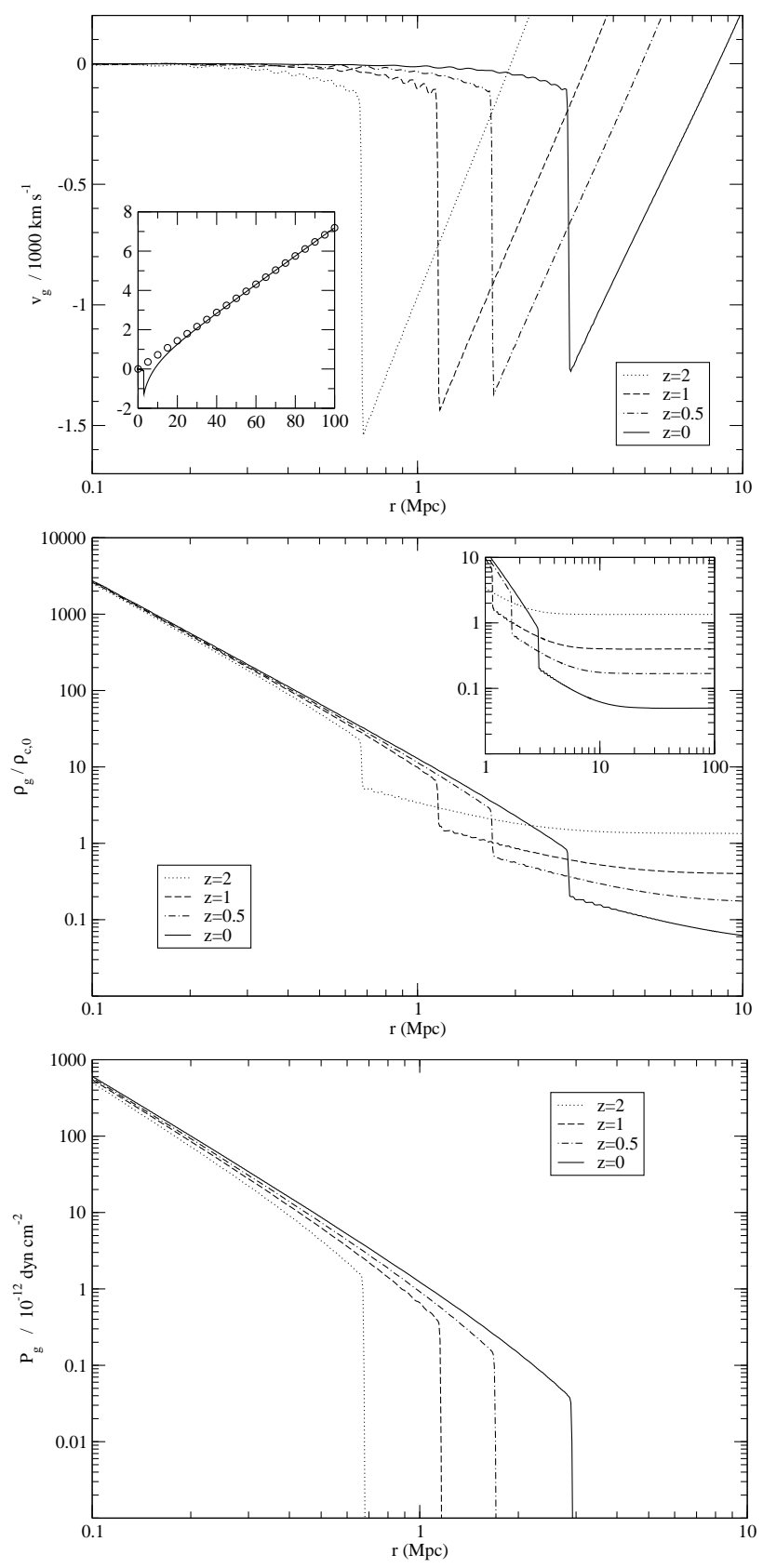

Fig. 3.1.- Gas velocity (upper panel), density (middle panel), and pressure (lower panel) profiles of our simulated cluster for the self-similar collisionless dark matter dominated accretion model in the Einstein-de Sitter universe at four different redshifts. The model has a dark matter mass accreted within $R_{178}$ of $M_{178}=10^{15} M_{\odot}$ at $z=0$. The insets in the upper and middle panels show the large radius behavior of the gas, with the velocity profile shown for $z=0$ only. The circles on the inset of the velocity profile give the Hubble flow velocity at $z=0$. We set $\Omega_{b}=0.05$ in this model. 

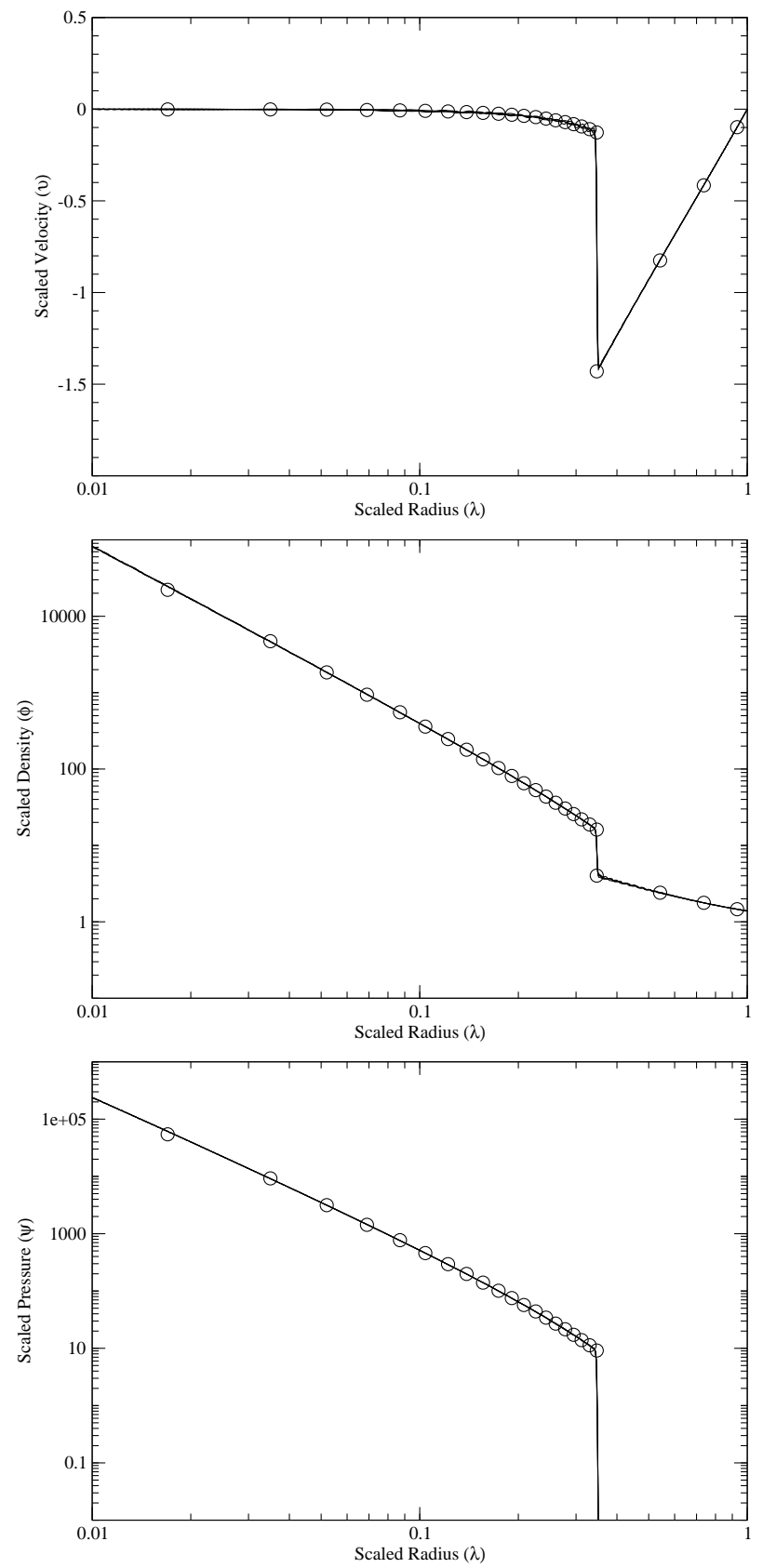

Fig. 3.2.- Scaled gas velocity (upper panel), density (middle panel), and pressure (lower panel) profiles of our simulated cluster. The hydrodynamic model is the same as in Figure 3.1. All four lines lie almost on top of one another in the figures. Circles show the corresponding analytic solutions. 

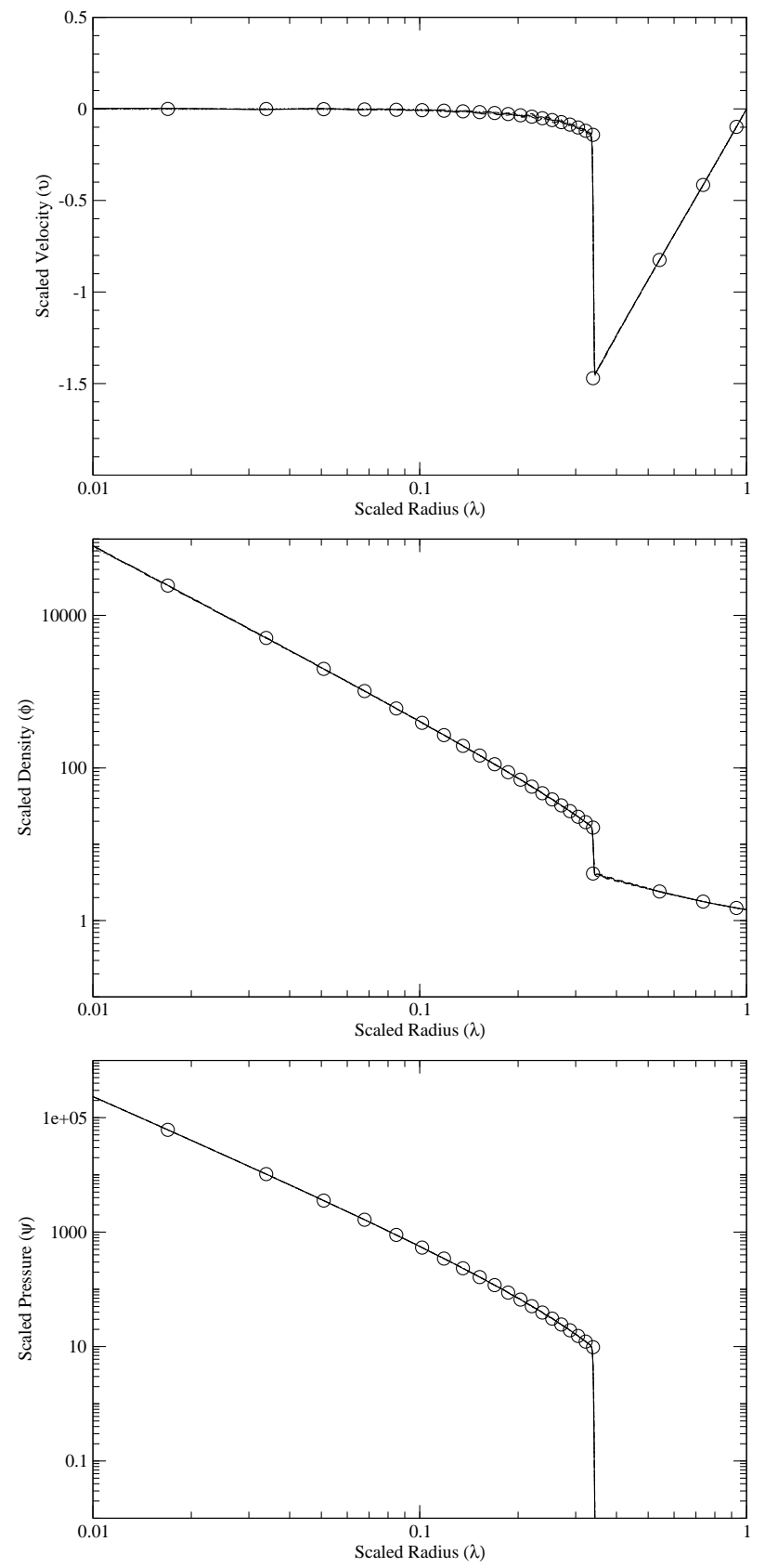

Fig. 3.3.- Similar to Figure 3.2 but for the self-similar collisional gas dominated accretion model in the Einstein-de Sitter universe. The model has a gas mass accreted within $R_{178}$ of $M_{178}=1.18 \times 10^{15} M_{\odot}$ at $z=0$. 


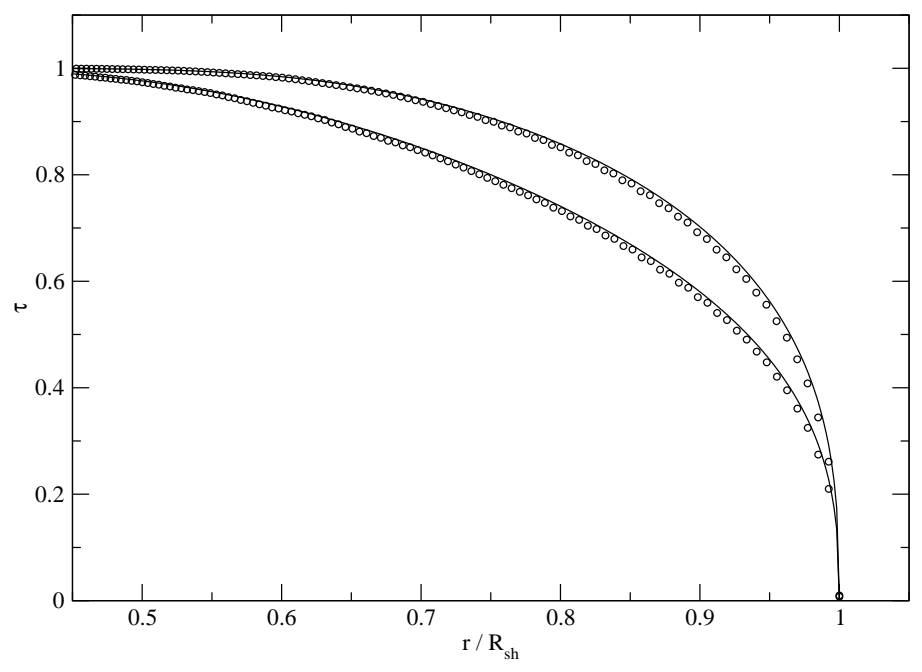

Fig. 3.4.- Ratio of the electron and average thermal dynamic temperature, $\tau$, as a function of scaled radius $r / R_{\mathrm{sh}}$ for clusters of the self-similar collisionless dark matter dominated accretion model in the Einstein-de Sitter universe at $z=0$. The upper and lower solid lines are the analytic solutions for cluster masses with $M_{178}=$ $5.26 \times 10^{14} M_{\odot}$ and $M_{178}=1.05 \times 10^{15} M_{\odot}$, respectively. The open circles on the corresponding lines are our simulated results. We assume $\Omega_{b}=0.05$ here. 

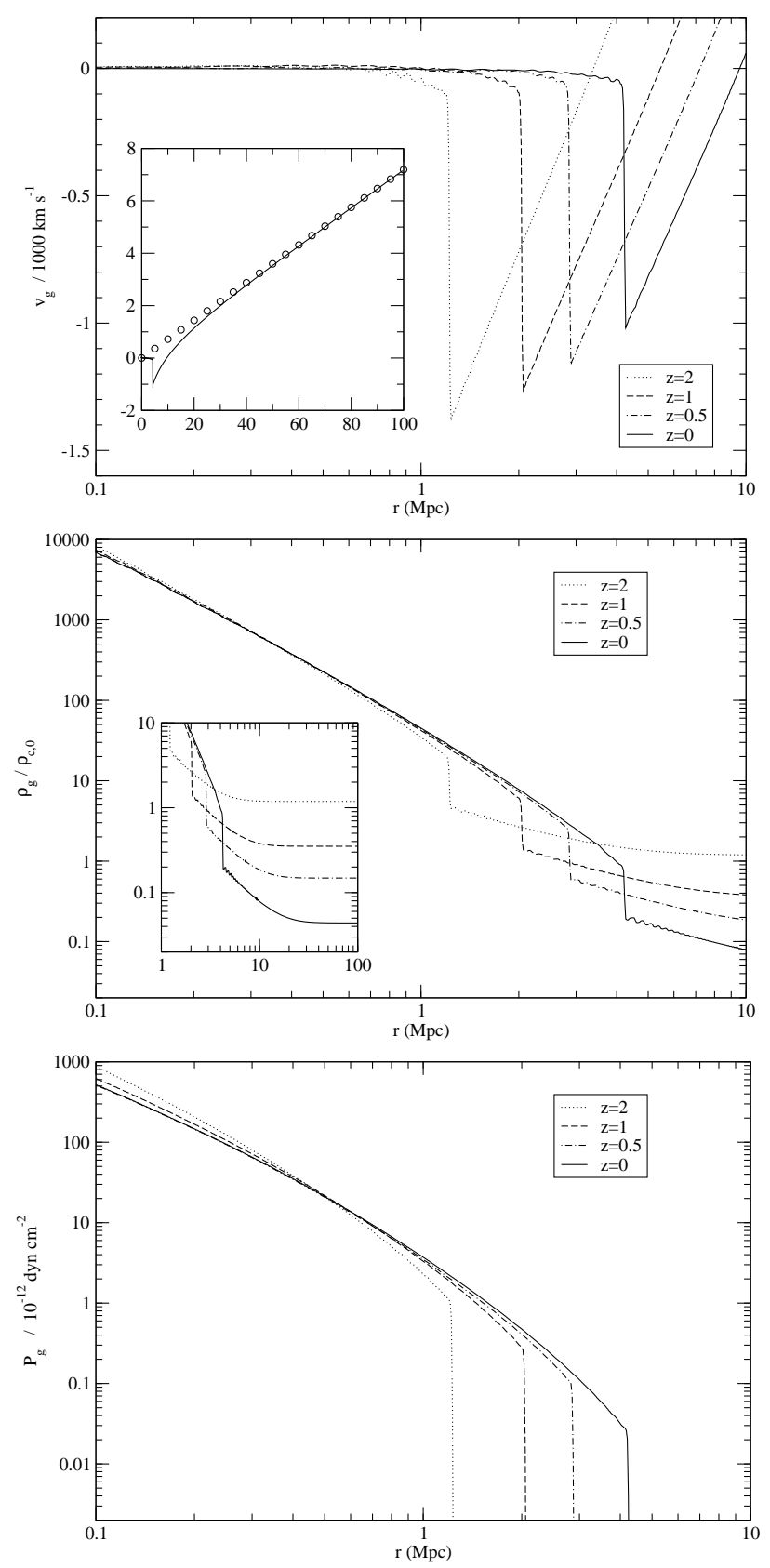

Fig. 3.5.- Gas velocity (upper panel), density (middle panel), and pressure (lower panel) profiles of our simulated cluster for the realistic NFW-dark energy model in the standard $\Lambda$ CDM universe at four different redshifts. The model has total mass accreted within $R_{\mathrm{sh}}$ of $M_{\mathrm{sh}}=1.53 \times 10^{15} M_{\odot}$ at $z=0$. The insets show the large radius behavior of the gas, with the velocity profile shown for $z=0$ only. The circles on the inset of the velocity profile give the Hubble flow velocity at $z=0$. 


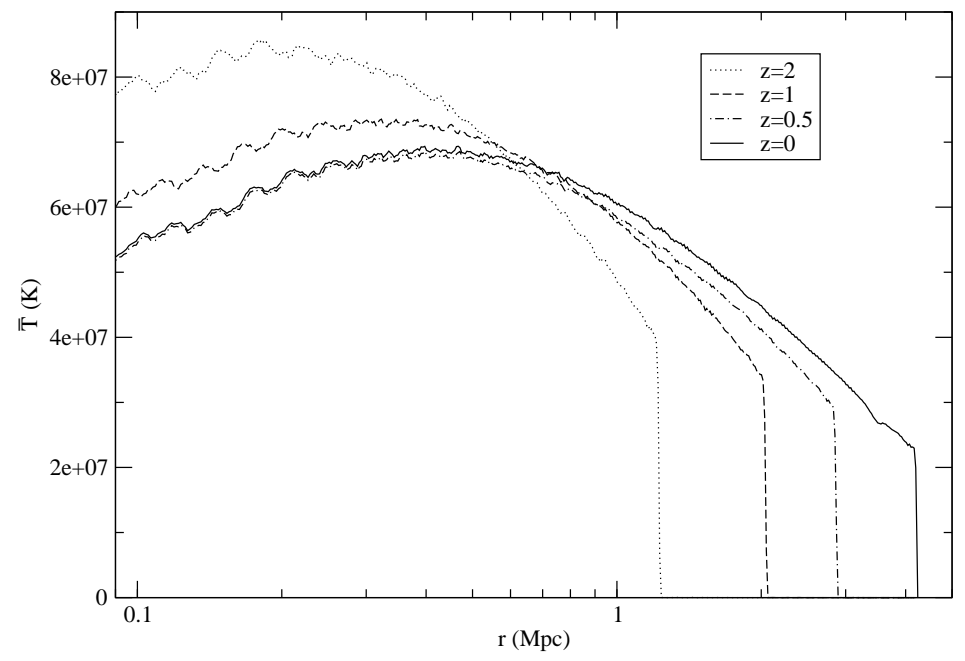

Fig. 3.6.- Average thermodynamic temperature profiles of our simulated realistic NFW-dark energy model cluster. The hydrodynamic model is the same as in Figure 3.5.

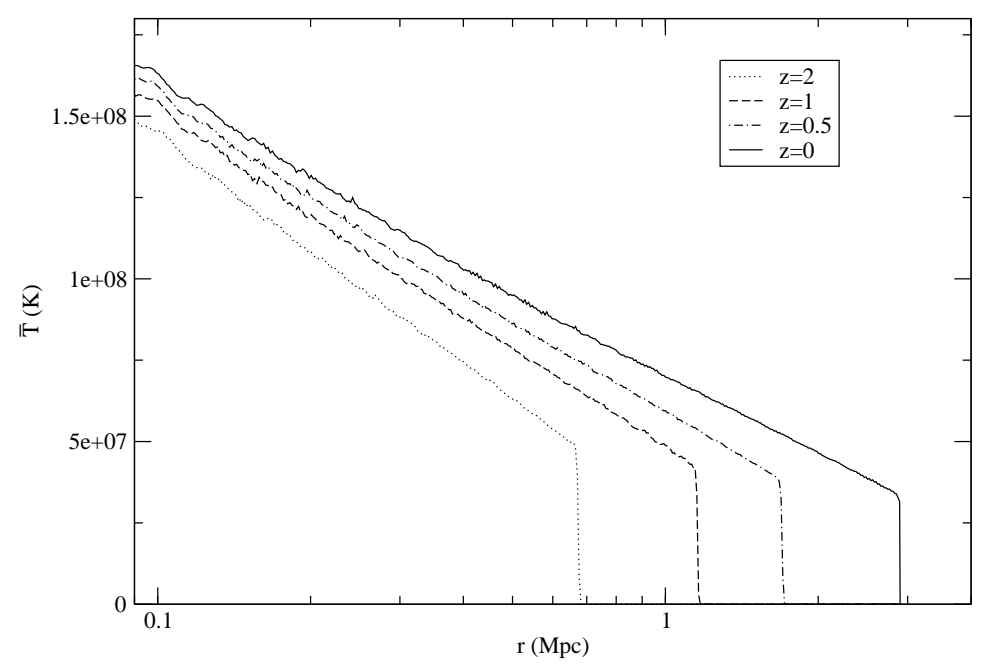

Fig. 3.7. - Average thermodynamic temperature profiles of our simulated self-similar dark matter dominated cluster. The hydrodynamic model is the same as in Figure 3.1. 


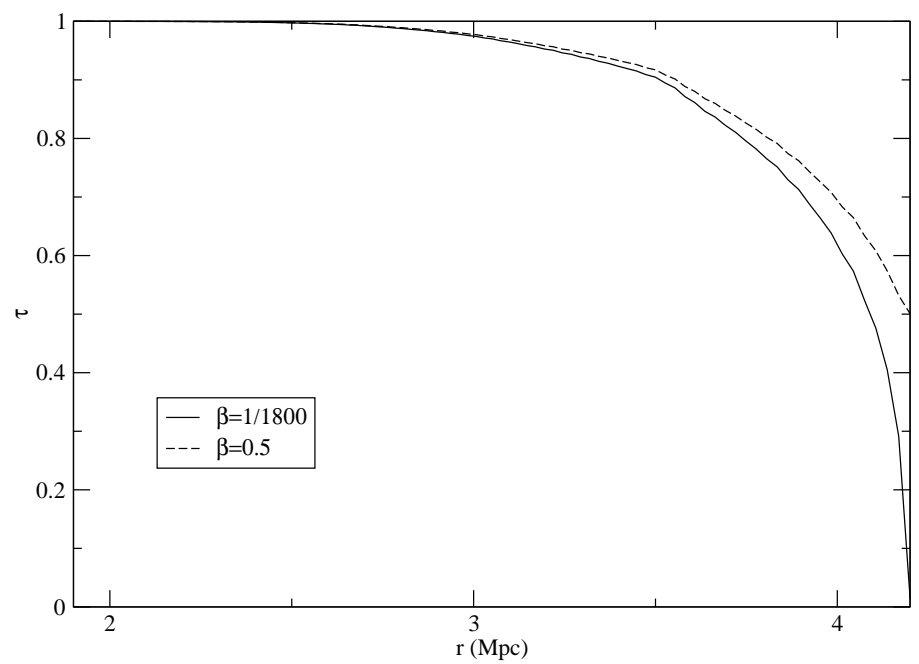

Fig. 3.8.- Ratio of electron and average thermodynamic temperatures, $\tau \equiv T_{e} / \bar{T}$, as a function of radius at $z=0$. The hydrodynamic model is the same as in Figure 3.5. The models with shock heating efficiency $\beta=1 / 1800$ and 0.5 are shown in solid and dashed lines, respectively.

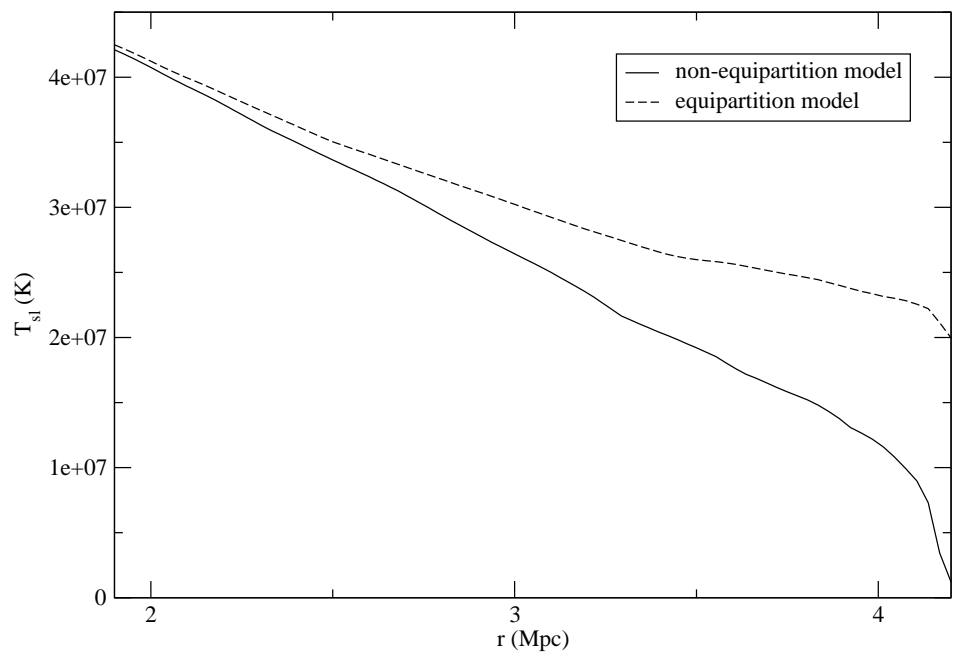

Fig. 3.9.- Projected X-ray spectroscopic-like temperature profiles for cluster models at $z=0$. The hydrodynamic model is the same as in Figure 3.5. The nonequipartition model with $\beta=1 / 1800$ is shown in solid line, while the equipartition model is shown in dashed line. We assume $Z=0.3 Z_{\odot}$ here. 


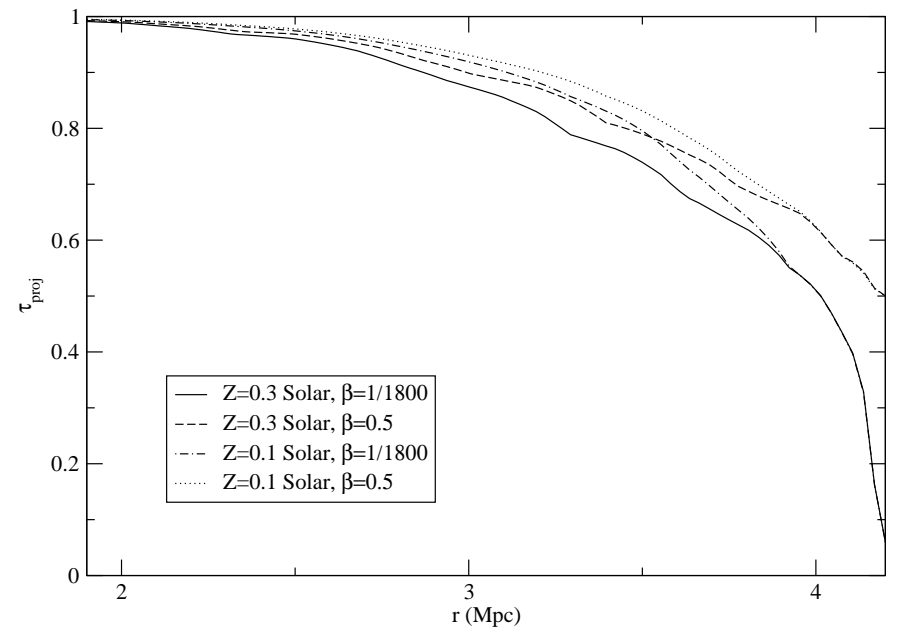

Fig. 3.10.- Ratio of the projected X-ray spectroscopic-like temperature profiles of the non-equipartition (with $\beta=1 / 1800$ and 0.5 ) and the equipartition models at $z=0$. Models with $Z=0.1$ and $0.3 Z_{\odot}$ are presented. The hydrodynamic model is the same as in Figure 3.5.

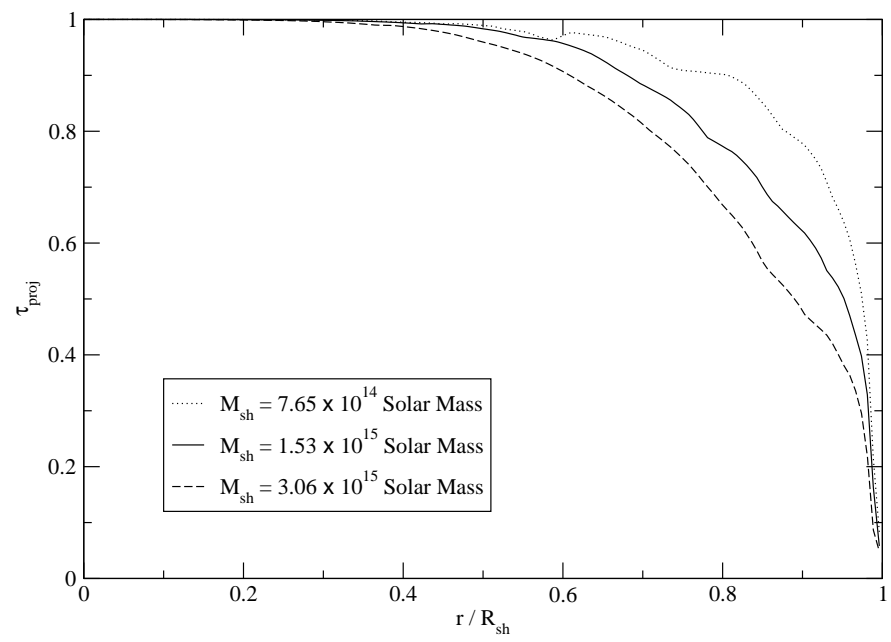

Fig. 3.11. - Ratio of the projected X-ray spectroscopic-like temperature of the nonequipartition with $\beta=1 / 1800$ and the equipartition models as a function of $r / R_{\mathrm{sh}}$ at $z=0$ for different cluster masses. The hydrodynamic models are similar to those in Figure 3.5, but with $M_{\mathrm{sh}}=7.65,15.3$, and $30.6 \times 10^{14} M_{\odot}$ at $z=0$. We assume $Z=0.3 Z_{\odot}$ here. 


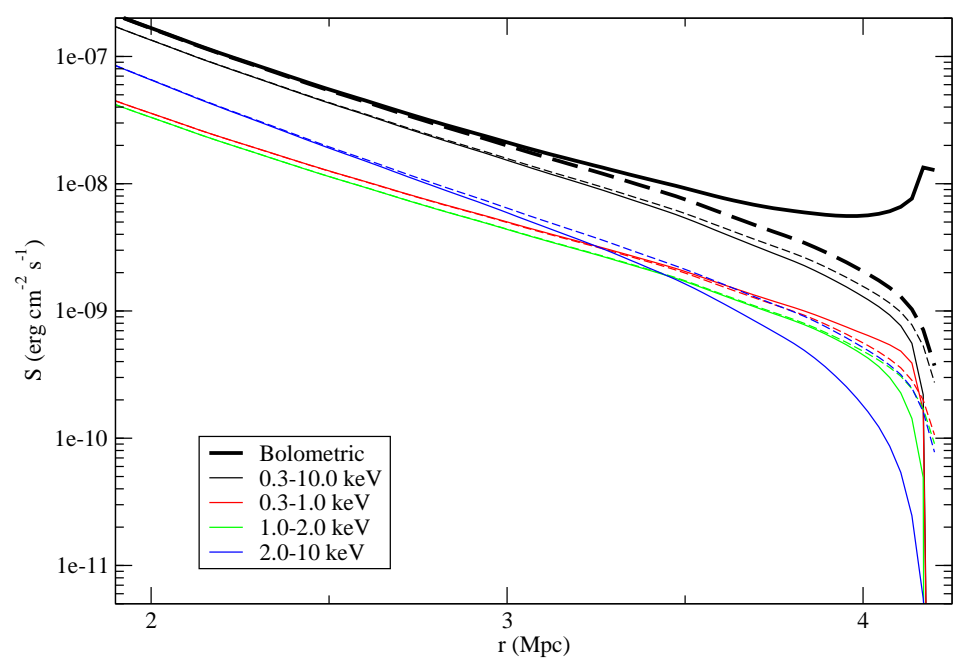

Fig. 3.12. - Surface brightness profiles for various energy bands for the nonequipartition (solid lines) and equipartition (dashed lines) cluster models at $z=0$. Models are the same as in Figure 3.9.

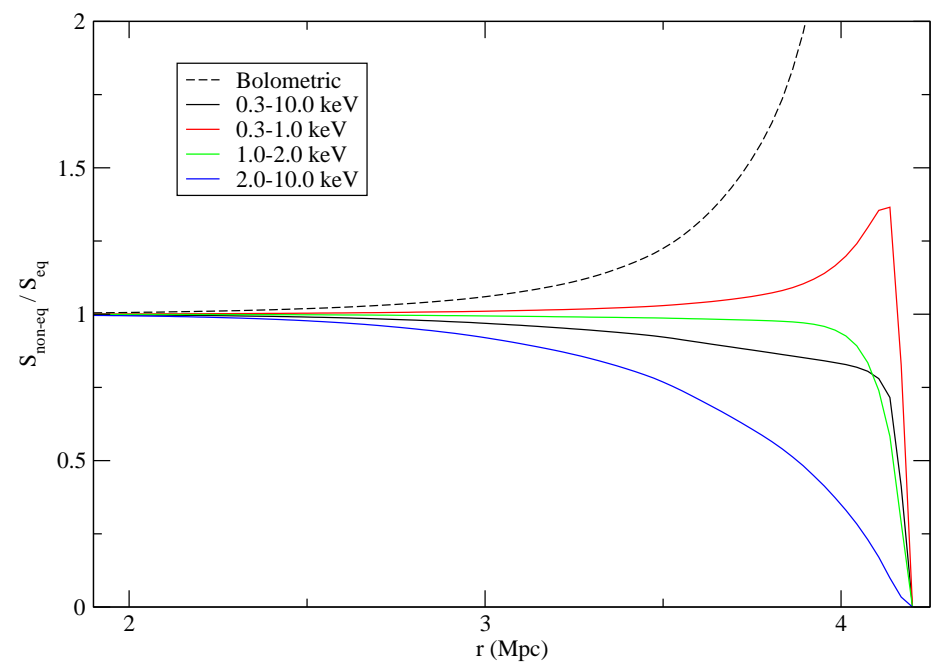

Fig. 3.13.- Ratios $S_{\text {non-eq }} / S_{\text {eq }}$ as a function of radius. Models are the same as in Figure 3.12. The ratio of the bolometric surface brightness near the shock radius reaches $\sim 35$ (outside the scale of the figure). 


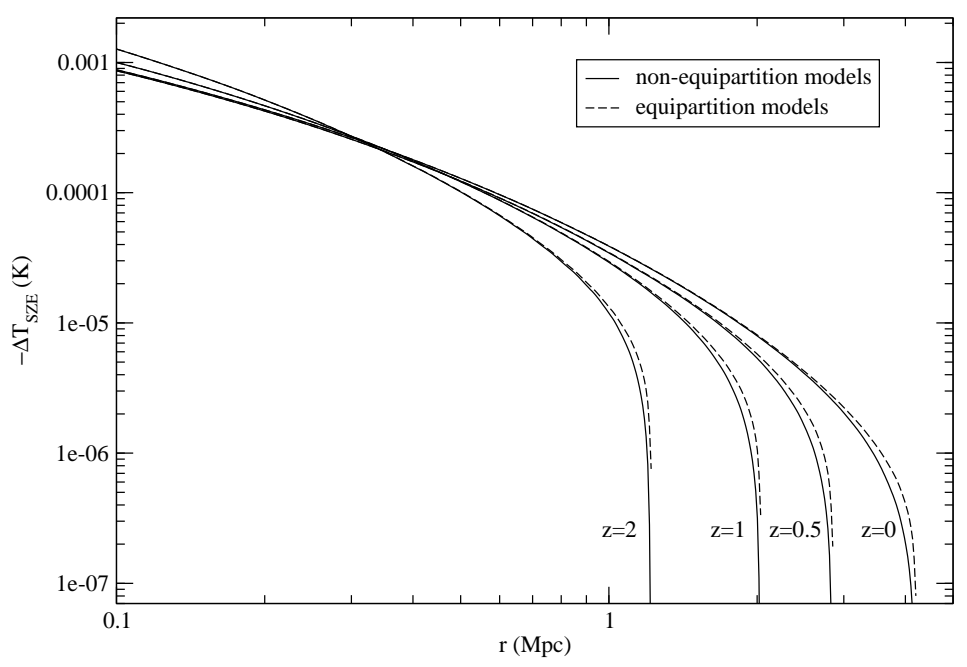

Fig. 3.14. - SZ temperature decrement profiles, $-\Delta T_{\mathrm{SZE}}$, of our simulated cluster at four different redshifts. The non-equipartition models $(\beta=1 / 1800)$ are shown in solid lines, while the equipartition models are shown in dashed lines. The hydrodynamic model is the same as in Figure 3.5.

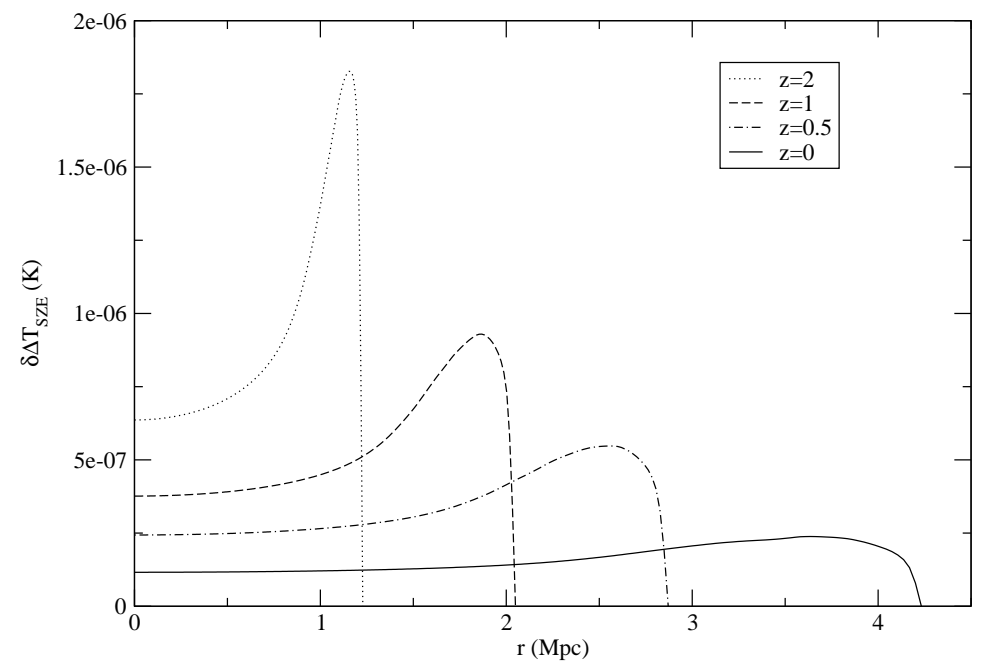

Fig. 3.15. - Difference $\delta \Delta T_{\mathrm{SZE}}$ between the SZ temperature decrements of the equipartition and the non-equipartition models $(\beta=1 / 1800)$ at four different redshifts. Models are the same as in Figure 3.14. 


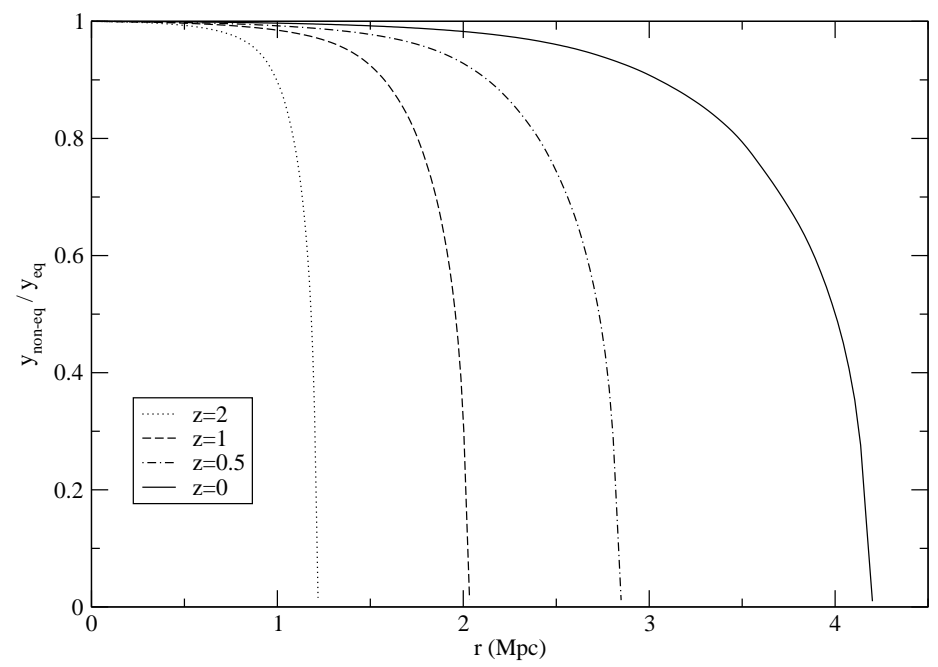

Fig. 3.16. - Ratio $y_{\text {non-eq }} / y_{\text {eq }}$ between the Comptonization parameters of the equipartition and the non-equipartition models $(\beta=1 / 1800)$ at four different redshifts. Models are the same as in Figure 3.14.

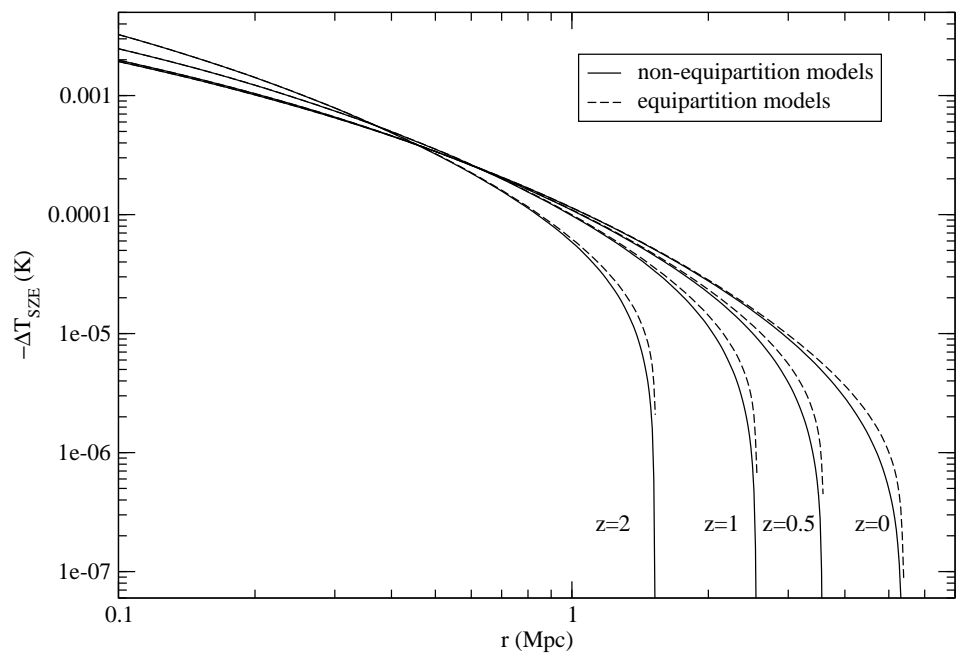

Fig. 3.17. - Same as Figure 3.14 but with $M_{\mathrm{sh}}(z=0)=3.06 \times 10^{15} M_{\odot}$ for the cluster model. 


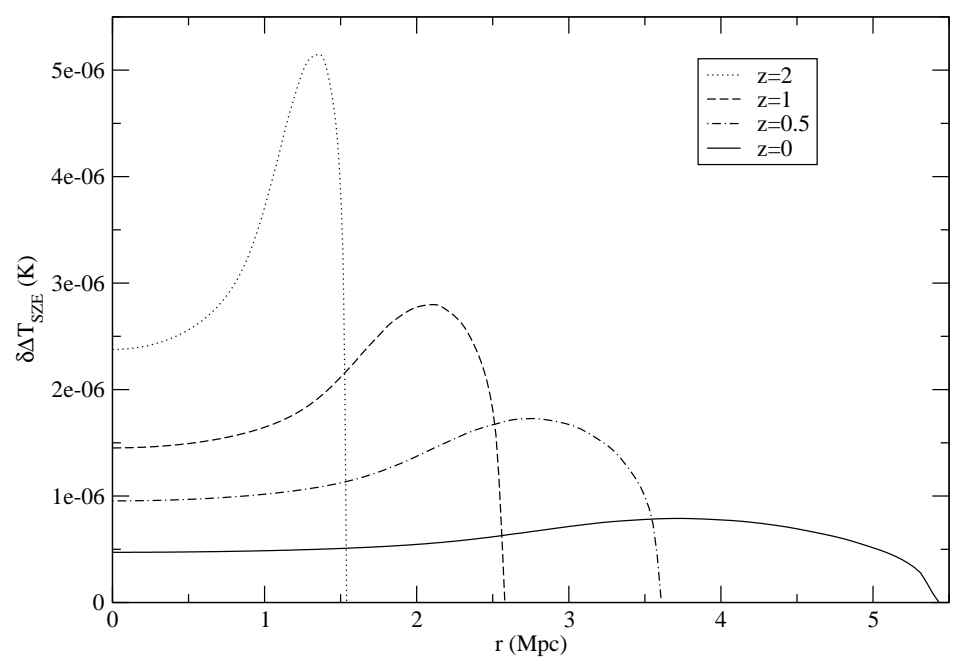

Fig. 3.18. - Same as Figure 3.15 but with $M_{\mathrm{sh}}(z=0)=3.06 \times 10^{15} M_{\odot}$ for the cluster model.

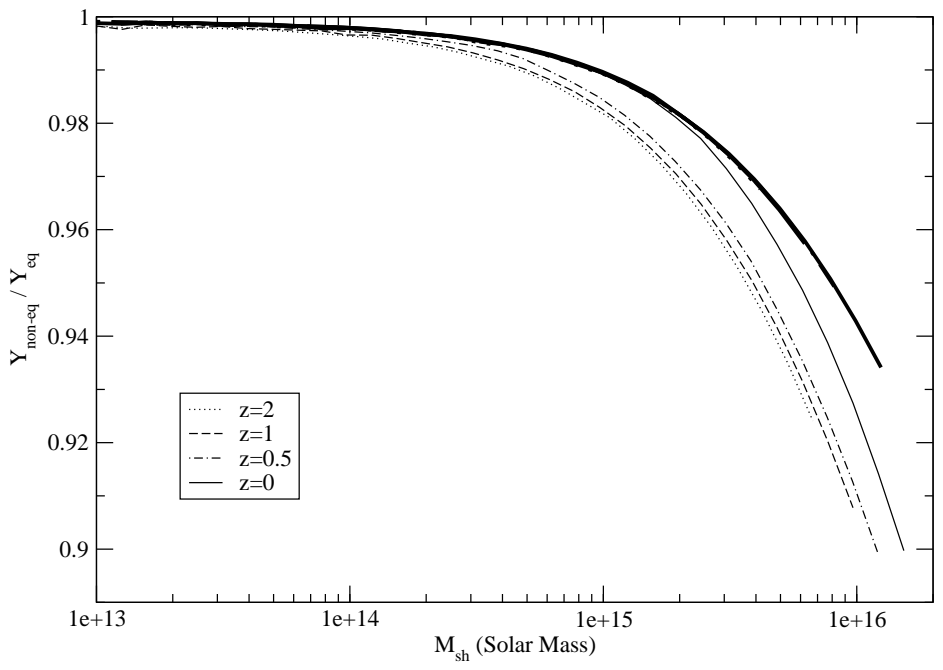

Fig. 3.19. - Integrated SZ biases, $Y_{\text {non-eq }} / Y_{\text {eq }}$, as a function of $M_{\text {sh }}$ for both our simulated realistic NFW model in the $\Lambda$ CDM universe (thin lines) and the numerical simulated self-similar model in the Einstein-de Sitter universe (thick lines) at four different redshifts. We assume $f_{\text {gas }}=0.17$ for models in the Einstein-de Sitter universe. The four lines for the self-similar model lie almost along the same line which cannot be easily distinguished on the graph. 


\section{Chapter 4}

\section{The Impact of Non-Equipartition}

\section{on Cosmological Parameter}

\section{Estimation from}

\section{Sunyaev-Zel'dovich Surveys}

[Adapted from Wong, K.-W., Sarazin, C. L., \& Wik, D. R. 2010, ApJ, 719, 1]

\subsection{Introduction}

Observational and theoretical studies have shown that galaxy clusters can be used as cosmological probes. In particular, the evolution of the galaxy cluster abundance, or the mass function, is sensitive to cosmological parameters including the average matter density $\Omega_{M}$, the normalization of the power spectrum of the initial density fluctuations $\sigma_{8}$, and the dark energy equation of state parameter $w$ (Mantz et al. 2008; Vikhlinin et al. 2009). Except for gravitational lensing which is difficult to do 
for large sample of galaxy clusters, the masses of galaxy clusters cannot be directly measured, and hence the mass function cannot be measured easily. Very often, the masses are estimated using mass proxies such as the Sunyaev-Zel'dovich (SZ) temperature distortion, the X-ray flux, or galaxy dynamics. The mass-observable relations have to be calibrated empirically or semi-empirically with numerical simulations (e.g., Vikhlinin et al. 2009). Hence, measuring cosmological parameters using the galaxy cluster abundance requires a full understanding of mass-observable relations. Even if the mass function and the mass-observable relation can be fitted simultaneously ("self-calibration"; Levine et al. 2002; Hu 2003; Majumdar \& Mohr 2003), the correct form of the mass-observable relation is needed.

A recent X-ray survey has shown that even a sample of only 85 X-ray clusters is sufficient to provide very tight constraints on some cosmological parameters. For example, $\sigma_{8}$ can be measured down to $1 \%$ level in statistical uncertainty using the cluster abundance technique alone by assuming a flat universe with fixed dark energy equation of state parameter and a prior on the Hubble constant (Vikhlinin et al. 2009). However, the statistical uncertainties on some other cosmological parameters (e.g., $\Omega_{M}$ ) are still slightly larger than $10 \%$. Ongoing and future SZ surveys will detect thousands of clusters (e.g., Birkinshaw 1999; Carlstrom et al. 2002; Bartlett et al. 2008), and this will significantly improve the constraints on cosmological parameters. Therefore, it is important to control the systematic uncertainties of galaxy cluster physics at even a percentage level.

Because of the very long Coulomb collisional timescale in the low-density outer regions of galaxy clusters, it has been pointed out that electron and ions there can be in non-equipartition (Fox \& Loeb 1997; Ettori \& Fabian 1998). Numerical simulations have shown that the SZ observables, e.g., integrated Comptonization parameter 
$(Y)$, for relaxed clusters can be biased by a few percent (Wong \& Sarazin 2009), and can potentially be biased up to $\sim 10 \%$ in major merging clusters (Rudd \& Nagai 2009). Specifically, the non-equipartition effect reduces the electron pressure compared to equipartition models, and hence the integrated Comptonization parameter of the non-equipartition model is smaller than that of the equipartition model. Although the uncertainties are still large, recent X-ray observations suggest that the electron pressure in cluster outer regions may be lower than that predicted by numerical simulations assuming equipartition (George et al. 2009; Basu et al. 2010; Hoshino et al. 2010). Recent observations of the secondary cosmic microwave background anisotropies with the South Pole Telescope (SPT) and the WMAP 7 year data also suggest that the electron pressure is smaller than the value predicted by hydrodynamic simulations (Lueker et al. 2009; Komatsu et al. 2010). These observational signatures are consistent with electrons and ions in non-equipartition, although it is also possible that the hydrodynamic simulations may simply overestimate the gas pressure. Another possibility is that heat conduction outside the clusters may be reducing the gas pressure (Loeb 2002).

In Chapter 3, we have shown that the non-equipartition effect can introduce biases in the integrated SZ effect, and the biases depend on cluster mass and evolve with redshift in the $\Lambda \mathrm{CDM}$ cosmology. The non-equipartition model was discussed in detail in Wong \& Sarazin (2009). In this chapter, we study the impact of non-equipartition effect on precision cosmology studies using the non-equipartition models we have developed. We consider only the non-equipartition effect associated with an accretion shock at the outer edge of a cluster. No significant collisionless electron heating at the accretion shocks is assumed, and the intergalactic gas outside the collisionless accretion shock is taken to be cold. These assumptions maximize the non-equipartition 
effects of the accretion shock. On the other hand, cluster mergers are not considered in our work. Mergers may increase the non-equipartition effect by a few percent, and hence our calculations of the biases in cosmological parameter estimation may still underestimate these effects. However, the non-equipartition effect induced by mergers lasts for only $0.5-1$ Gyr (Rudd \& Nagai 2009) which is comparable to the timescale that mergers can temporarily enhance the integrated SZ effect by boosting the overall temperature (Wik et al. 2008, hereafter WSR). Such transient phenomenon will mainly introduce scatter in the $Y$ versus mass relation, and the effect on cosmological parameter estimation is small in general. The non-equipartition effect induced by mergers may partially cancel out the merger boost in SZ effect, and hence the merger effect on cosmological parameter estimation may even be smaller. On the other hand, the non-equipartition effects in the accretion shock regions can systematically bias the $Y$ versus mass relation for all clusters, as long as clusters are continuously accreting materials from the surrounding which is believed to be generally true. Moreover, systematic uncertainties in precision cosmology using galaxy clusters can be minimized by restricting the sample of clusters to the highest degree of dynamical relaxation, and hence considering the systematic effects on relaxed clusters alone is particularly important. We follow Randall et al. (2002, hereafter RSR) and WSR closely to quantify the biases in cosmological parameter estimation using semi-analytical techniques. Specifically, we study the non-equipartition effect on the $Y$ versus mass relation (Section 4.2). Such a biased $Y$ versus mass relation will affect the number of clusters with $Y$ observed in SZ surveys, i.e., the $Y$ function (Section 4.3). In this work, the $Y$ function is calculated using the extended Press-Schechter theory (Press \& Schechter 1974). We consider three cases which may potentially introduce biases in the $Y$ versus mass relations if the non-equipartition effect is not properly taken into ac- 
count during the cross-calibration or self-calibration processes when using the galaxy cluster abundance technique (Section 4.4.1). We quantify and discuss the impact on cosmological parameter estimation from SZ surveys by fitting the mass function with the biased $Y$ versus mass relations (Section 4.4.2). Section 4.5 gives the discussion and conclusions. Throughout the chapter, we assume the Hubble constant $H_{0}=71.9 h_{71.9} \mathrm{~km} \mathrm{~s}^{-1} \mathrm{Mpc}^{-1}$ with $h_{71.9}=1$.

\subsection{SZ Versus Mass Correlation}

The SZ effect can be characterized as the Comptonization parameter, $y$, which is given by

$$
y=\frac{k_{B} \sigma_{\mathrm{T}}}{m_{e} c^{2}} \int n_{e} T_{e} d l \propto \int P_{e} d l,
$$

where $\sigma_{\mathrm{T}}$ is the Thomson scattering cross section, $n_{e}$ is the electron number density, $T_{e}$ is the electron temperature, $P_{e}=n_{e} k_{B} T_{e}$ is the electron pressure, and $l$ is the distance along the line of sight. The integrated Comptonization parameter, $Y$, is defined as the integral of the Comptonization parameter in equation (4.1) over the area of the cluster on the sky

$$
Y=d_{A}^{2} \int y d \Omega=\int y d A
$$

where $d_{A}$ is the angular diameter distance to the cluster, $\Omega$ is the solid angle of the cluster on the sky, and $A$ is the projected surface area. In this chapter, $Y$ is integrated over the projected surface area of the cluster out to the shock radius. This quantity is useful for spatially unresolved clusters with SZ observations where the beam area covers the whole cluster.

It has been shown that the integrated Comptonization parameter displays a tight 
correlation with cluster mass (Reid \& Spergel 2006). Such a tight correction is useful for precision cosmology, and hence a correct understanding of the integrated Comptonization parameter is important. A detailed discussion of the use of SZ surveys to study cosmology can be found in Carlstrom et al. (2002). In this chapter, we assume the SZ effect versus mass relation for the equipartition model to be the same as the equilibrium $Y-M$ relation used in WSR. Specifically, the equipartition SZ effect versus mass relation we assume is of the form

$$
Y_{\text {eq }}=N x^{\alpha} p[x] h_{71.9}^{-2} \mathrm{Mpc}^{2},
$$

where $x=M_{200} /\left(h_{71.9}^{-1} 10^{15} M_{\odot}\right), N$ is the normalization constant, $\alpha$ is the powerlaw index, and $p[x]$ is a 13 degree polynomial in $x$. Equation (4.3) is fitted to the numerical solutions for the equilibrium $Y-M$ relation in WSR. The integrated SZ bias introduced by the non-equipartition effect, $Y_{\text {non-eq }} / Y_{\text {eq }}$ versus $M$ at different redshifts, is taken from Wong \& Sarazin (2009). The non-equipartition SZ effect versus mass relation we used in this chapter is hence given by

$$
Y_{\text {non-eq }}=Y_{\text {eq, } \mathrm{WsR}} \times\left(\frac{Y_{\text {non-eq }}}{Y_{\text {eq }}}\right)_{\mathrm{ws}},
$$

where the subscripts "WSR" and "WS" here indicate that the terms are taken from different models in WSR and Wong \& Sarazin (2009), respectively. In this work, since we are interested in the relative effects on SZ surveys and cosmological parameter estimation introduced by the non-equipartition effects instead of the precise $Y-M$ relation which depends on details of numerical simulations, in principle, we can take any equilibrium $Y-M$ relation from simulations and apply our non-equipartition bias to the equilibrium $Y-M$ relation. The reason for using the equilibrium $Y-M$ relation 
in WSR to model the non-equipartition $Y-M$ relation instead of the $Y-M$ relation in Wong \& Sarazin (2009) is that the former relation takes into account the dependence of $Y$ on gas fraction $f_{\text {gas }}$, where $f_{\text {gas }} \propto M^{1 / 3}$ for $M_{200} \gtrsim 10^{14} M_{\odot}$. The numerical solutions in Wong \& Sarazin (2009) assume a constant $f_{\text {gas }}$, and a self-similar argument shows that $Y \propto M^{5 / 3} f_{\text {gas }} \propto M^{5 / 3}$. The equilibrium $Y-M$ relation in WSR has a power-law index close to 2 . On the other hand, the non-equipartition effects on the integrated $Y$ depend weakly on $f_{\text {gas }}$ and hence we can assume the $Y$ bias of the constant $f_{\text {gas }}$ models in Wong \& Sarazin (2009) can be applied to the varying $f_{\text {gas }}$ model in WSR. Another advantage of using the equilibrium $Y-M$ relation in WSR is that we can compare the effect of non-equipartition on cosmological parameters estimations to the merger effects calculated in WSR. The equipartition $Y-M$ relation and the integrated SZ bias introduced by the non-equipartition effect we used in this chapter are plotted in Figure 4.1. Clusters with higher masses are hotter, and hence, the equipartition timescales are longer. Thus, the non-equipartition effects are stronger in more massive clusters (Fox \& Loeb 1997; Wong \& Sarazin 2009). For our non-equipartition model in the $\Lambda \mathrm{CDM}$ universe, the integrated SZ bias decreases as redshift decreases. This is probably due to the decreasing rate of accretion onto clusters in the $\Lambda \mathrm{CDM}$ universe during the cosmological acceleration, which results in a longer time for electron-ion equilibration (Wong \& Sarazin 2009).

In order to quantify the effect of non-equipartition on the SZ versus mass relation, we fit a power-law function to the integrated SZ bias of the form

$$
\left(Y_{\text {non-eq }} / Y_{\text {eq }}\right)_{\mathrm{WS}}=\Delta N\left(\frac{M_{200}}{10^{15} M_{\odot}}\right)^{\Delta \alpha}
$$

to all the clusters with $M_{200}$ between $10^{14}$ and $4 \times 10^{15} M_{\odot}$. The range is consistent with the cluster mass range used to study the mass function in X-ray observations 
(Vikhlinin et al. 2009). We also fit over the wider range of $10^{13}$ to $10^{16} M_{\odot}$ for comparison. The fitted coefficients $\Delta N$ and $\Delta \alpha$ correspond to the biases in the fitted SZ versus mass relation if the non-equipartition effect is not taken into account. The clusters in our sample are distributed roughly uniformly in the logarithm of the mass. The fitted results are listed in Table 4.1.

The effect of non-equipartition is to decrease $Y$ for the high-mass clusters, and hence the effect on the SZ versus mass relation is to lower the power-law index by 0.01 to 0.016 , which corresponds to a decrease of $0.5 \%-0.8 \%$ for the SZ versus mass relation with power-law index $\alpha=2$. This is comparable to the $0.8 \%$ uncertainty of the power-law index derived from simulations combined with observations within $M_{500}$ (Arnaud et al. 2009). The normalization is biased to compensate for the change in power-law index, and the bias is about $3 \%$ for the scaled mass of $10^{15} M_{\odot}$. For SZ surveys which measure $Y$ out to the shock radius, e.g., observations with a spatial resolution poorer than typical shock radii, we have shown that the non-equipartition effect can introduce a small deviation in the measured $Y-M$ relation. The deviations are small, but future SZ surveys with sufficient statistics may be able to detect such signatures. On the other hand, if clusters are spatially resolved and $Y$ are measured within $R_{200}$, we have shown that non-equipartition effect is smaller than $1 \%$ for all clusters, and hence the bias in the $Y-M$ relation is negligible (Wong \& Sarazin 2009). A bias in measurement out to the shock radii but not within $\sim R_{200}$ will indicate that cluster outer regions may be in non-equipartition.

\subsection{Effects of Non-Equipartition on SZ Surveys}

The number of galaxy clusters expected to be found per unit comoving volume depends sensitively on cosmology. The quantity which is convenient to describe the 
cluster number density is the mass function, $n(M, z)$, where $n(M, z) d M$ gives the number of clusters per unit comoving volume with masses in the range $M \rightarrow M+d M$, and $z$ is the redshift. While the exact form of the mass function can be found most accurately from cosmological simulations (Springel et al. 2005), a semi-analytic form of the mass function given by the extended Press-Schechter theory (Press \& Schechter 1974; Bond et al. 1991; Lacey \& Cole 1993) provides a more convenient way to understand the dependence of the mass function on cosmological parameters, especially when we are interested in the relative effect instead of the precise values of the mass function itself. Although the Press-Schechter theory cannot reproduce the mass function found in cosmological simulations at very high redshifts and low cluster masses (Sheth \& Tormen 1999; Lukić et al. 2007), it is more than sufficient over the redshifts ( $z=0$ to 2$)$ and masses $\left(M=10^{14}\right.$ to $\left.10^{16} M_{\odot}\right)$ of interest here.

The mass function given by the extended Press-Schechter theory can be written as (Press \& Schechter 1974)

$$
n_{\mathrm{PS}}(M, z) d M=\sqrt{\frac{2}{\pi}} \frac{\bar{\rho}}{M} \frac{\delta_{c}(z)}{\sigma^{2}(M)}\left|\frac{d \sigma(M)}{d M}\right| \exp \left[-\frac{\delta_{c}^{2}(z)}{2 \sigma^{2}(M)}\right] d M
$$

where $\bar{\rho}$ is the current mean of the total mass density of the universe, $\sigma(M)$ is the current rms density fluctuation within a sphere of mean mass $M$, and $\delta_{c}(z)$ is the critical linear overdensity required for a region to collapse at redshift $z$. Unless otherwise specified, the parameters used in this chapter are the same as those in RSR and WSR.

Once the $Y$ versus mass relation is known, the distribution function of $Y$ is given by the $Y$ function,

$$
n_{\mathrm{PS}}(Y, z)=n_{\mathrm{PS}}(M, z) \frac{d M}{d Y},
$$


where $n_{\mathrm{PS}}(Y, z) d Y$ gives the number of clusters per unit comoving volume at redshift $z$ which have the integrated SZ parameters in the range $Y \rightarrow Y+d Y$. The $Y$ function for the non-equipartition models and the equipartition models can be related by

$$
n_{\mathrm{PS}, \text { non-eq }}\left(Y_{\text {non-eq }}, z\right)=n_{\mathrm{PS}, \mathrm{eq}}\left(Y_{\mathrm{eq}}, z\right) \frac{d Y_{\mathrm{eq}}}{d Y_{\text {non-eq }}}
$$

where the subscripts "eq" and "non-eq" denote the equipartition and the non-equipartition models, respectively.

Figure 4.2 shows the $Y$ functions for the equipartition and the non-equipartition models and also their ratios at different redshifts for the standard $\Lambda$ CDM cosmology. The theoretical $Y$ functions can be biased strongly for large $Y$ and high redshift clusters. In practice, whether the bias can affect the observed $Y$ functions depends on the number of clusters that can be observed, and this depends on cosmology. The maximum number of clusters that can be observed with $Y$ values in the range $Y=Y \rightarrow Y+d Y$ and with redshifts in the range $z=z \rightarrow z+d z$ is $n_{\mathrm{PS}}(Y, z) d Y d V$, where $d V$ is the comoving volume of the universe between redshifts $z$ and $z+d z$. For each of the four redshifts $z=0,0.5,1.0$, and 2.0, we selected a redshift interval $z_{l}$ to $z_{u}$ as given in the second and third columns of Table 4.2. Then, we defined values of $Y_{0}$ and $n_{\mathrm{PS}, 0}$ such that $\int_{Y_{0}}^{\infty} \int_{z_{l}}^{z_{u}} n_{\mathrm{PS}}(Y, z) d Y(d V / d z) d z=1$ and $n_{\mathrm{PS}, 0}(z)=$ $n_{\mathrm{PS}}\left(Y_{0}, z\right)$ for the equipartition models. These values are listed in Table 4.2 and are plotted in Figure 4.2 as solid dots. For example, between $z=0.25 \rightarrow 0.75$ for the assumed cosmology, the expected number of clusters with $Y \gtrsim 6 \times 10^{-4} h_{71.9}^{-2} \mathrm{Mpc}^{2}$ $\left(M \gtrsim 2 \times 10^{15} h_{71.9}^{-1} M_{\odot}\right)$ is one. On the other hand, the expected number of clusters with $Y=(1 \rightarrow 2) \times 10^{-4} h_{71.9}^{-2} \operatorname{Mpc}^{2}\left[M=(0.9 \rightarrow 1.3) \times 10^{15} h_{71.9}^{-1} M_{\odot}\right]$ is about 200 within the same redshifts interval, and the bias in $n_{\mathrm{PS}}$ is about $5 \%$. 


\subsection{Effects of Non-Equipartition on Cosmological Parameter Estimation from SZ Surveys}

In this section, we follow a procedure similar to that outlined in RSR and WSR to address the impact of non-equipartition effects on cosmological parameter estimation from SZ surveys. Readers who are interested in the technical details should refer to RSR and WSR. We outline the fitting procedure used in this work and address the difference between the previous works below.

As discussed in Section 4.3, the mass function of galaxy clusters is sensitive to cosmology, and hence measuring the galaxy cluster abundance at different redshifts can provide constraints to cosmological parameters. In particular, the properties of the dark energy can potentially be determined (Haiman et al. 2001). However, the masses of galaxy clusters cannot be directly determined, and a mass proxy must be observed to determine the mass through the mass-observable relation. Examples of mass proxies are the SZ temperature distortion, the X-ray flux, and the weak leasing shear. If the mass-observable relations can be calibrated, this can provide very tight constraints on cosmological parameters (Mantz et al. 2008; Vikhlinin et al. 2009). The mass-observable relations can be calibrated by numerical simulations and/or cross-calibrations with some other observables, and these are subjected to systematic uncertainties due to cluster physics and/or observational constraints. On

the other hand, the mass-observable relations can be simultaneously fitted with the mass function of galaxy clusters, and this is called "self-calibration" (Levine et al. 2002; Hu 2003; Majumdar \& Mohr 2003). The sensitivity in constraining cosmological parameters and the mass-observable relations by "self-calibration" depends on both the form of the mass-observable relations and the mass function of galaxy clusters. In 
this work, we are mainly interested in the biases introduced by the non-equipartition effect, and the effects on the mass-observable relations. Hence, we assume there is no other systematic uncertainties in the mass function in any given cosmology.

\subsubsection{Systematic Uncertainties Introduced by Non-Equipartition}

To address the impact of non-equipartition effects on cosmological parameter estimation from SZ surveys, we quantify the impact as biases in the cosmological parameter estimates if the calibration of the $Y$ versus mass relation does not include the nonequipartition effect. We generate the integrated $Y$ function with the non-equipartition effects included under an assumed cosmology, and call the generated $Y$ function the non-equipartition $Y$ function. We then fit the non-equipartition $Y$ function with the incorrectly calibrated $Y$ versus mass relation. We consider three different cases for the incorrectly calibrated $Y$ versus mass relation. For the first case (Case 1), we assume the $Y$ versus mass relation is calibrated with incorrect numerical simulations that assume equipartition, and this incorrectly calibrated $Y$ versus mass relation is used to fit the mass function. An example of this systematic bias might occur if the $Y$ versus mass relation was extrapolated to the shock radius using observations within a smaller radius together with numerical simulations assuming equipartition. In this case, the integrated SZ bias is simply given by

$$
b_{1}=\left(\frac{Y_{\text {non-eq }}}{Y_{\text {eq }}}\right)_{\mathrm{WS}}
$$

where the right-hand side is the same term in equation (4.4).

For the second case (Case 2), we assume the $Y$ versus mass relation is self- 
calibrated by fitting the $Y$ versus mass relation and the mass function simultaneously, but with an incorrect functional form in the $Y$ versus mass relation. We assume the incorrect functional form of the $Y$ versus mass relation to be a power law in mass. In this case, we assume the integrated SZ bias is given by

$$
b_{2}=\left(\frac{Y_{\text {non-eq }}}{Y_{\text {eq }}}\right)_{\mathrm{WS}} /\left(\frac{Y_{\text {non-eq }}}{Y_{\text {eq }}}\right)_{\text {plfit }},
$$

where the term with the subscript "plfit" is the best-fit power-law relation given in equation (4.5).

For the third case (Case 3), we assume the $Y$ versus mass relation is calibrated correctly at $z=0$, but the $Y$ versus mass relation at higher redshifts is incorrectly calibrated by extrapolating the calibration from that at $z=0$. In this case, we assume the integrated $\mathrm{SZ}$ bias is given by

$$
b_{3}=\left(\frac{Y_{\text {non-eq }}}{Y_{\mathrm{eq}}}\right)_{\mathrm{WS}, z} /\left(\frac{Y_{\text {non-eq }}}{Y_{\mathrm{eq}}}\right)_{\mathrm{WS}, z=0} .
$$

Studying the impact on cosmological parameter estimation for all of these cases is equivalently to fitting the non-equipartition SZ luminosity function by the equipartition SZ luminosity function in equation (4.8), but replacing the $d Y_{\text {eq }} / d Y_{\text {non-eq }}$ by $b_{1}, b_{2}$, and $b_{3}$ in equations $(4.9)-(4.11)$.

\subsubsection{Fitting Procedures and Results}

For each case of the systematic bias we studied, we generate the $Y$ function with the non-equipartition bias included as given in equation (4.8) by assuming the standard $\Lambda$ CDM cosmological model with $\Omega_{M}=0.258, \sigma_{8}=0.796$, and a constant dark energy equation of state parameter $w=-1$. The $Y$ function generated can be directly 
calculated from the analytic Press-Schechter mass function in equation (4.6) and the biased $Y$ versus mass relations. This is simpler than those in RSR or WSR where merger trees were needed to generate the mass function in order to follow the merger history. In our work, all the $Y$ versus mass relations are biased regardless of the merger history. We then fit the generated $Y$ function with an equipartition model and find the best-fit values of the cosmological parameters. Clusters of galaxies can be used to constrain the dark energy equation of state parameter, $w$. Even the evolution of $w$ can potentially be constrained. In this work, we study the constraint on $w(z)$ using the form $w=w_{0}+w_{1} z /(1+z)^{2}$; the detailed explanation of this choice can be found in WSR. We consider three cases when fitting the cosmological parameters: i) fitting the $\Omega_{M}$ and $\sigma_{8}$ but fixing $w(z)=-1$; ii) fitting the $\Omega_{M}, \sigma_{8}$, and assuming $w(z)=w_{0}$, where $w_{0}$ is a constant to be fitted; and iii) fitting the $\Omega_{M}, \sigma_{8}$, and $w=w_{0}+w_{1} z /(1+z)^{2}$, where $w_{0}$ and $w_{1}$ are constants to be fitted. The $Y$ functions are simultaneously fitted at four different redshifts $(z=0,0.5,1.0$, and 2.0) to break the degeneracy in the fitted cosmological parameters. We choose only to fit $Y$ between $5 \times 10^{-6} h_{71.9}^{-2} \mathrm{Mpc}^{2}$ and $5 \times 10^{-3} h_{71.9}^{-2} \mathrm{Mpc}^{2}$. The lower limit is chosen because clusters are likely to be confused in the SZ surveys for $M \lesssim 10^{14} h_{71.9}^{-1} M_{\odot}$ (Holder et al. 2007). Increasing the lower limit will make the biases in cosmological parameters larger since the non-equipartition effect increases with cluster mass. The upper limit corresponds roughly to the most massive cluster that can be formed in the $\Lambda \mathrm{CDM}$ universe. The limits are also consistent with SZ surveys being done or planned (e.g., Melin et al. 2005). We also limit the fits to values of $Y<Y_{0}$ and $z$ such that the maximum number of observable clusters $\int_{Y_{0}}^{\infty} \int_{z_{l}}^{z_{u}} n_{\mathrm{PS}}(Y, z) d Y(d V / d z) d z \gtrsim 1$ for most redshift bins (Table 4.2).

Non-equipartition $Y$ functions at several redshifts and fitted models are shown in 
Figure 4.3. The deviations in the non-equipartition and the fitted $Y$ functions are small and only visible in the residual plots. In Case 1 and Case 2 with the dark energy equation of state parameter frozen at $w=-1$, for $z \leq 1$, the non-equipartition $Y$ functions are higher than the best-fitted $Y$ functions for low-mass clusters, and the opposite is true for high-mass clusters. At $z=2$, the non-equipartition $Y$ function is slightly higher than the best-fitted one in Case 1 with $w$ frozen at $w=-1$, but the opposite is true in Case 2. The residual is similar in appearance if we free the constant value of $w$ ( $w_{1}$ frozen at zero) or allow $w$ to vary with redshift in Case 1 and Case 2. In Case 3, the residual is more complicated. The residuals are of the order of a few percent, and this will affect the estimated cosmological parameters as discussed below.

The fitted results of the cosmological parameters for different cases we considered are summarized in Table 4.3. In general, for all three systematic uncertainty cases we studied, when freezing $w=-1$, the deviations of $\Omega_{M}$ and $\sigma_{8}$ from the assumed cosmology are $\lesssim 1 \%$. The best-fitted parameters happen to be consistent with the assumed cosmology, but we can see from the residual plots that there are clear systematic deviations by a few percent (e.g., Figure 4.3). Such systematic deviations might be confused with the effect of non-Gaussian initial conditions on galaxy cluster mass functions, which also show similarly shaped systematic deviations (e.g., Figure 1 in Fedeli et al. 2009).

If we free the constant value of $w=w_{0}$, non-equipartition effects can be significant depending on how the calibration is done. For Case $1, \Omega_{M}, \sigma_{8}$, and $w_{0}$ now deviate by $+3.3 \%,-1.6 \%$, and $-5.4 \%$, respectively. This shows that ignoring the non-equipartition effects in the $Y$ versus mass relation when cross-calibrating with numerical simulations can introduce significant biases in cosmological parameter es- 
timations when one is trying to constrain the dark energy equation of state. Either self-calibrating using a power-law form in the $Y-M$ relation (Case 2) or calibrating the $Y-M$ relation correctly at low redshift (Case 3) can significantly reduce the biases in $\Omega_{M}, \sigma_{8}$, or $w_{0}($ down to $\lesssim 1 \%)$.

If we allow $w$ to vary with redshift, non-equipartition effects can introduce significant biases in cosmological parameter estimates (up to $\sim 10 \%$ ). Self-calibrating using a power-law form in the $Y-M$ relation (Case 2) can reduce the biases in $\Omega_{M}$ and $\sigma_{8}$ down to $\lesssim 1 \%$, but non-equipartition effects can still introduce a $\sim 4 \%$ bias in the constant normalization of the dark energy equation of state parameter $\left(w_{0}\right)$ and introduce an apparent evolution of the same order. Calibrating the $Y-M$ relation correctly at low redshift (Case 3) can further reduce the bias in $w_{0}$ to $\lesssim 1 \%$, but again, there is still an apparent evolution of $\sim 3 \%$. These results show that the mass function technique is very sensitive to dark energy, and a full understanding of the systematic uncertainties in galaxy cluster physics is essential to constrain the dark energy equation of state using the mass function technique.

\subsection{Discussion and Conclusions}

Numerical simulations have shown that the SZ observables (e.g., the integrated Comptonization parameter $Y$ ) for relaxed clusters can be biased by a few percent (Wong \& Sarazin 2009), and potentially up to $\sim 10 \%$ in major merging clusters (Rudd \& Nagai 2009). These results are consistent with the SPT and the WMAP 7 year data which indicate that electron pressures are smaller than predicted by hydrodynamic simulations (Lueker et al. 2009; Komatsu et al. 2010). A few X-ray observations in the cluster outer regions also show that the electron pressure is lower than the pressure predicted by numerical simulations which assume equipartition (George et al. 2009; 
Basu et al. 2010; Hoshino et al. 2010). However, it is also possible that numerical simulations simply overestimate the gas pressure, or that the gas is supported in part by other forces such as turbulent or cosmic ray pressure. The $Y-M$ relation can be biased if non-equipartition effects are not properly taken into account. Precision cosmological studies using the evolution of the galaxy cluster abundance rely on a full understanding of the mass-observable relations, if the mass of the galaxy clusters cannot be directly measured as is usually the case in practice. We have studied systematically the impact of biased $Y-M$ relation introduced by the non-equipartition effect on SZ surveys and on precision cosmological studies.

While previous studies show that the $Y-M$ relation is stable to complicated physical processes such as mergers and the power-law index in the $Y-M$ relation is robust (Poole et al. 2007; WSR), we have shown that non-equipartition effect can introduce a deviation from the power law in the $Y-M$ relation. We have fitted a power-law to the non-equipartition $Y-M$ relation, and we found that there is a $\sim 1 \%$ bias in the power-law index, which is comparable to the uncertainty of the power-law index derived from simulations combined with observations within $M_{500}$ (Arnaud et al. 2009). Such a small systematic bias and deviation from the power-law form have important implications for SZ surveys and precision cosmological studies using the SZ surveys.

Using the analytic extended Press-Schechter theory to quantify the mass function of galaxy clusters, we have studied the non-equipartition effects on SZ surveys. We found that the $Y$ functions can be biased strongly for large $Y$ and high-redshift clusters. For example, the expected number of clusters with $Y=(1 \rightarrow 2) \times 10^{-4} h_{71.9}^{-2} \mathrm{Mpc}^{2}$ $\left[M=(0.9 \rightarrow 1.3) \times 10^{15} h_{71.9}^{-1} M_{\odot}\right]$ between $z=0.25 \rightarrow 0.75$ can be biased by $\sim 5 \%$. The net effect is that ignoring non-equipartition effects underestimates the abundance of high-mass and high-redshift clusters. Cosmological parameters measured by using 
the cluster counting technique from SZ surveys will be biased if non-equipartition effects are not taken into account.

We have quantified the impact of non-equipartition effects on cosmological parameter estimations from SZ surveys by the galaxy cluster abundance technique using biased $Y-M$ relations. We considered three potential systematic biases in the $Y-M$ relations if the non-equipartition effect is not properly taken into account during the cross-calibration or self-calibration when using the galaxy cluster abundance technique. The best-fit cosmological parameters, $\Omega_{M}, \sigma_{8}$, and also the dark energy equation of state parameters $\left[w=w_{0}+w_{1} z /(1+z)^{2}\right]$ using the biased $Y-M$ relations were determined. For all the three methods of calibrating the $Y$-mass relation we have studied, if the dark energy equation of state parameter is frozen at $w=-1$, we find that the best-fit $\Omega_{M}$ and $\sigma_{8}$ are consistent with the assumed cosmology to within $\sim 1 \%$. However, there are clear systematic deviations of a few percent in the fitted $Y$ functions which may be confused with other effects such as non-Gaussian initial conditions (Fedeli et al. 2009). Models with non-Gaussian initial conditions predict that the actual number of clusters with higher mass can be lower than the models with Gaussian initial conditions (e.g., Figure 1 in Fedeli et al. 2009); our non-equipartition model predicts an apparent smaller $Y$ for high-mass clusters and this underestimates the number of high mass clusters if the non-equipartition effect is not taken into account. Note that at such small levels of systematic deviations, other systematic uncertainties such as the use of different mass functions (e.g., Press \& Schechter 1974; Sheth \& Tormen 1999) may introduce larger systematic biases. However, Fedeli et al. (2009) also used the Press-Schechter mass function (as we do) to determine the effect of non-Gaussianity. Thus, we can directly compare these effects with the results of non-equipartition, free of biases introduced by the choice of mass 
function, and estimate the bias non-equipartition will introduce in efforts to detect non-Gaussian fluctuations with clusters.

If $w$ is fitted as a constant parameter ( $w_{1}$ frozen at zero), then depending on the calibration methods, non-equipartition effect can introduce a few percent biases on the measured cosmological parameters (Case 1). Either self-calibrating the $Y-M$ relation using a power-law form (Case 2) or calibrating the $Y-M$ relation correctly at low redshift (Case 3) can significant reduce the biases in $\Omega_{M}, \sigma_{8}$, or $w_{0}$ to $\lesssim 1 \%$. If we allow $w$ to vary with redshift, the non-equipartition effect can introduce a bias in cosmological parameter of up to $\sim 10 \%$ (Case 1). In particular, non-equipartition effects can introduce an apparent evolution in $w$ of a few percent in all of the cases we considered.

Using the cluster abundance technique alone, an X-ray survey with 85 X-ray clusters has already constrained some cosmological parameters down to $1 \%$ level in statistical uncertainty (Vikhlinin et al. 2009). Ongoing and future SZ surveys will detect thousands of clusters (e.g., Birkinshaw 1999; Carlstrom et al. 2002; Bartlett et al. 2008), and this will significantly improve the constraints on cosmological parameters. Therefore, it is important to control the systematic uncertainties of galaxy cluster physics at even a percentage level. Hydrodynamic simulations assuming equipartition suggest that the integrated $Y$ is a robust mass proxy even when galaxy clusters are in the process of merging, and hence the integrated $Y$ is taken to be a nearly ideal probe for cosmological studies (Poole et al. 2007; WSR).

Our results show that if the non-equipartition effect is not properly taken into account, cosmological parameters can be biased significantly (up to $\sim 10 \%$ ). In order to take the non-equipartition effect into account when using cluster abundance to study precision cosmology, the ultimate solution is to include the non-equipartition 
effect in cosmological simulations assuming the non-equipartition physics is known accurately. If higher resolution is needed, another approach is to correct the nonequipartition effect by performing idealized simulations (e.g., Wong \& Sarazin 2009) or to re-simulate representative clusters taken from cosmological simulations including the non-equipartition effect with realistic assumptions. For the latter case, the non-equipartition effect can be taken into account together with other physical processes (e.g., gas depletion processes during the formation) which may also affect the $Y$ versus mass relation. However, the above calibration methods by numerical simulations rely on the assumption that the non-equipartition physics is known accurately, which is in fact not the case at present. One of the key systematic uncertainties is the electron heating efficiency at the collisionless shock, $\beta$. One way to constrain the non-equipartition physics is to make direct observations of accretion shocks, which is currently not feasible. We may constrain non-equipartition physics based on observations of other astrophysical shocks such as mergers shocks and supernova remnants. However, we have to assume these results apply to cluster accretion shocks, which may or may not be the case. Another route might be to perform plasma simulations (e.g., particle-in-cell simulations) to constrain the shock physics. However, to apply the plasma simulation results to cluster accretion shocks, a detailed knowledge of the pre-shock physics such as the magnetic field structure might be needed. Clearly, all of the above calculations are necessary to determine the range of the systematic uncertainties and the effects of the non-equipartition physics, and to constrain the form of the $Y$ versus mass relation. These should be studied in the near future. Until numerical simulations can directly determine the effects of non-equipartition on the $Y$ versus mass relation from first principles, we suggest either to self-calibrate the $Y$ versus mass relation using a power-law form at each redshift bin (Case 2), or to cali- 
brate the $Y$ versus mass relation correctly at low redshift (Case 3). These will reduce the biases due to non-equipartition on $\Omega_{M}, \sigma_{8}$, or $w_{0}$ to better than $1 \%$. Important biases introduced by other physical processes can be corrected in addition to the nonequipartition correction. However, if one would try to constrain the evolution in $w$ to better than $1 \%$, together with the self-calibration method, the constraints from numerical simulations with uncertainties less than a percent level might be necessary.

We have shown that using the cluster abundance to constraint the dark energy equation of state requires a full understanding of the systematic uncertainties in galaxy cluster physics. Even though we are only considering the systematic uncertainties introduced by the non-equipartition effect, our results also suggest that systematic uncertainties in the $Y-M$ relation introduced by other physics of even a few percent can introduce a comparable level of biases in cosmological parameter measurements. Future cluster surveys aiming to constrain departures from general relativity will need to control systematic uncertainties down to a sub-percentage level (Schmidt et al. 2009), and hence cluster physics must be understood in a comparable accuracy. Future theoretical calculations and numerical simulations should pay particular attention to the effects of non-thermal physics on the electron pressure profiles. Potential systematic uncertainties include conduction, turbulent pressure, magnetic pressure, and relativistic pressure supported by cosmic rays. Deep observations should also be carried out to constrain all these effects in detail for individual clusters. The outer regions of galaxy clusters are ideal sites for study non-thermal physics. These studies not only can increase our understanding of cosmology, but also can provide information on the physics of galaxy clusters and plasma physics under extreme conditions. 
Table 4.1. Effects of Non-Equipartition on the SZ-Mass Relation

\begin{tabular}{cccccc}
\hline \hline & \multicolumn{2}{c}{$(1-40) \times 10^{14} M_{\odot}$} & & \multicolumn{2}{c}{$(0.1-100) \times 10^{14} M_{\odot}$} \\
\cline { 2 - 3 } \cline { 5 - 5 }$z$ & $\Delta N$ & $\Delta \alpha$ & & $\Delta N$ & $\Delta \alpha$ \\
\hline 0.0 & 0.977 & -0.0126 & & 0.967 & -0.0117 \\
0.5 & 0.971 & -0.0164 & & 0.963 & -0.0122 \\
1.0 & 0.971 & -0.0152 & & 0.964 & -0.0109 \\
2.0 & 0.970 & -0.0158 & & 0.969 & -0.0085 \\
\hline
\end{tabular}

Table 4.2. Characteristic Numbers of Clusters

\begin{tabular}{cccccc}
\hline \hline$z$ & $z_{l}$ & $z_{u}$ & $Y_{0}\left(h_{71.9}^{-2} \mathrm{Mpc}^{2}\right)$ & $M_{0}\left(h_{71.9}^{-1} 10^{15} M_{\odot}\right)$ & $n_{\mathrm{PS}, 0}\left(h_{71.9}^{5} \mathrm{Mpc}^{-5}\right)$ \\
\hline 0.0 & 0.00 & 0.25 & $6.10 \times 10^{-4}$ & 2.27 & $4.81 \times 10^{-6}$ \\
0.5 & 0.25 & 0.75 & $5.95 \times 10^{-4}$ & 2.24 & $6.01 \times 10^{-8}$ \\
1.0 & 0.75 & 1.25 & $1.97 \times 10^{-4}$ & 1.26 & $4.99 \times 10^{-8}$ \\
2.0 & 1.25 & 2.75 & $6.04 \times 10^{-5}$ & 0.68 & $2.85 \times 10^{-10}$ \\
\hline
\end{tabular}

Note. - The values of $Y_{0}$ and $n_{\mathrm{PS}, 0}$ are defined such that $\int_{Y_{0}}^{\infty} \int_{z_{l}}^{z_{u}} n_{\mathrm{PS}}(Y, z) d Y(d V / d z) d z=1$ and $n_{\mathrm{PS}, 0}(z)=n_{\mathrm{PS}}\left(Y_{0}, z\right)$ for the equipartition models for different redshift intervals $z_{l}$ to $z_{u}$. The value of $M_{0}$ corresponding to $Y_{0}$ is related by equation (4.3). 
Table 4.3. Best-Fit Cosmological Parameters and Biases for Different $Y$ Versus Mass Calibrations

\begin{tabular}{ccccc}
\hline \hline Calibration & $\Omega_{M}$ & $\sigma_{8}$ & $w_{0}$ & $w_{1}$ \\
\hline \multirow{2}{*}{ Case 1 } & $0.2548(-1.2 \%)$ & $0.7950(-0.1 \%)$ & {$[-1]$} & {$[0]$} \\
& $0.2665(+3.3 \%)$ & $0.7830(-1.6 \%)$ & $-0.9464(-5.4 \%)$ & {$[0]$} \\
& $0.2680(+3.9 \%)$ & $0.7811(-1.9 \%)$ & $-0.9031(-9.7 \%)$ & $-0.2362(+5.9 \%)$ \\
\hline Case 2 & $0.2579(0 \%)$ & $0.7976(+0.2 \%)$ & {$[-1]$} & {$[0]$} \\
& $0.2602(+0.9 \%)$ & $0.7951(-0.1 \%)$ & $-0.9890(-1.1 \%)$ & {$[0]$} \\
& $0.2610(+1.2 \%)$ & $0.7940(-0.3 \%)$ & $-0.9577(-4.2 \%)$ & $-0.1725(+4.3 \%)$ \\
\hline Case 3 & $0.2585(+0.2 \%)$ & $0.7953(-0.1 \%)$ & {$[-1]$} & {$[0]$} \\
& $0.2604(+0.9 \%)$ & $0.7932(-0.4 \%)$ & $-0.9910(-0.9 \%)$ & {$[0]$} \\
& $0.2601(+0.2 \%)$ & $0.7938(-0.3 \%)$ & $-1.0123(+1.2 \%)$ & $0.1167(-2.9 \%)$ \\
\hline
\end{tabular}

Note. - The assumed correct cosmological parameters are $\Omega_{M}=0.258, \sigma_{8}=$ $0.796, w_{0}=-1$, and $w_{1}=0$. The bracketed values are the frozen values in the fits. The values in parentheses in Columns 2-4 are the percentage deviations of the fitted cosmological parameters from the assumed parameters. The values in parentheses in Column 5 are the largest percentage change in $w$ between the present time $(z=0)$ and $z=2$; this change is $\Delta w=w_{1} / 4$ assuming $w(z)=w_{0}+w_{1} z /(1+z)^{2}$. 


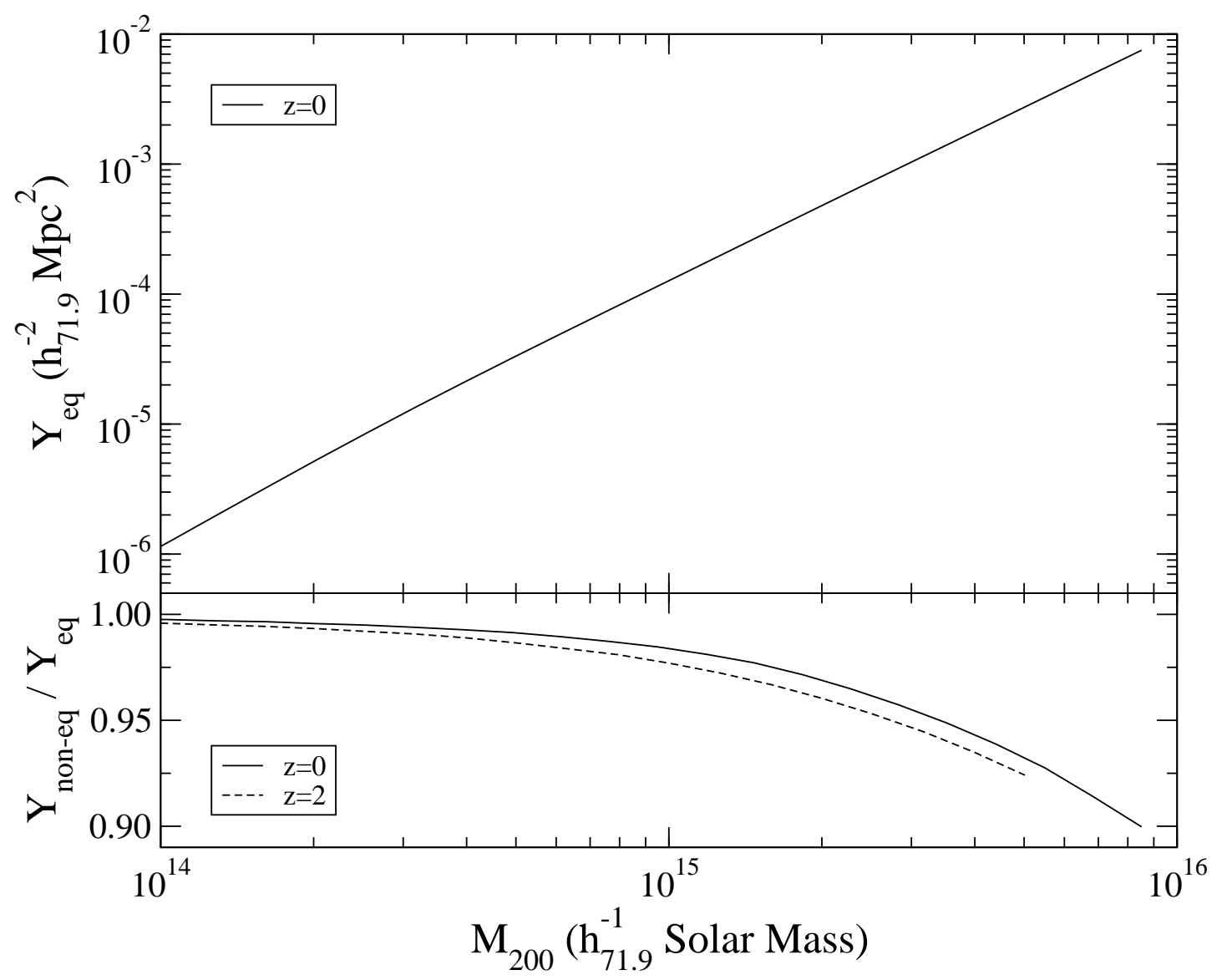

Fig. 4.1. - Top panel: Integrated Comptonization parameter $Y$ versus mass at $z=0$ for the equipartition model used in this chapter. Bottom panel: Integrated $Y$ bias, $\left(Y_{\text {non-eq }} / Y_{\text {eq }}\right)_{\mathrm{ws}}$, versus mass at $z=0$ and 2. 


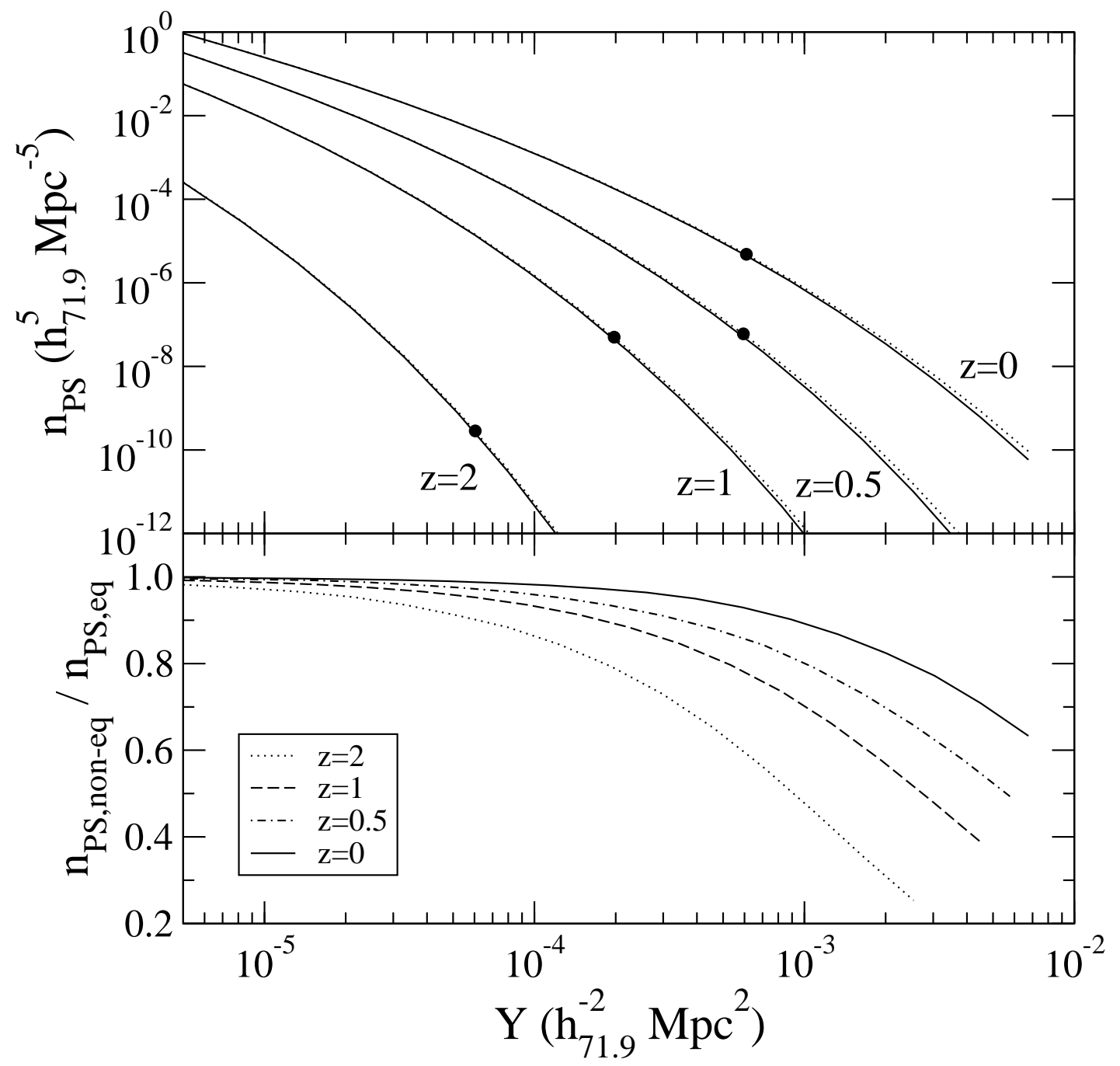

Fig. 4.2.- Top panel: Integrated $Y$ functions of the non-equipartition (solid lines) and the equipartition (dotted lines) models in the standard $\Lambda$ CDM universe. Solid dots indicate the values of $Y_{0}$ such that $\int_{Y_{0}}^{\infty} \int_{z_{l}}^{z_{u}} n_{\mathrm{PS}}(Y, z) d Y(d V / d z) d z=1$ for the equipartition model. Bottom panel: Ratios between the non-equipartition and equipartition $Y$ functions. 


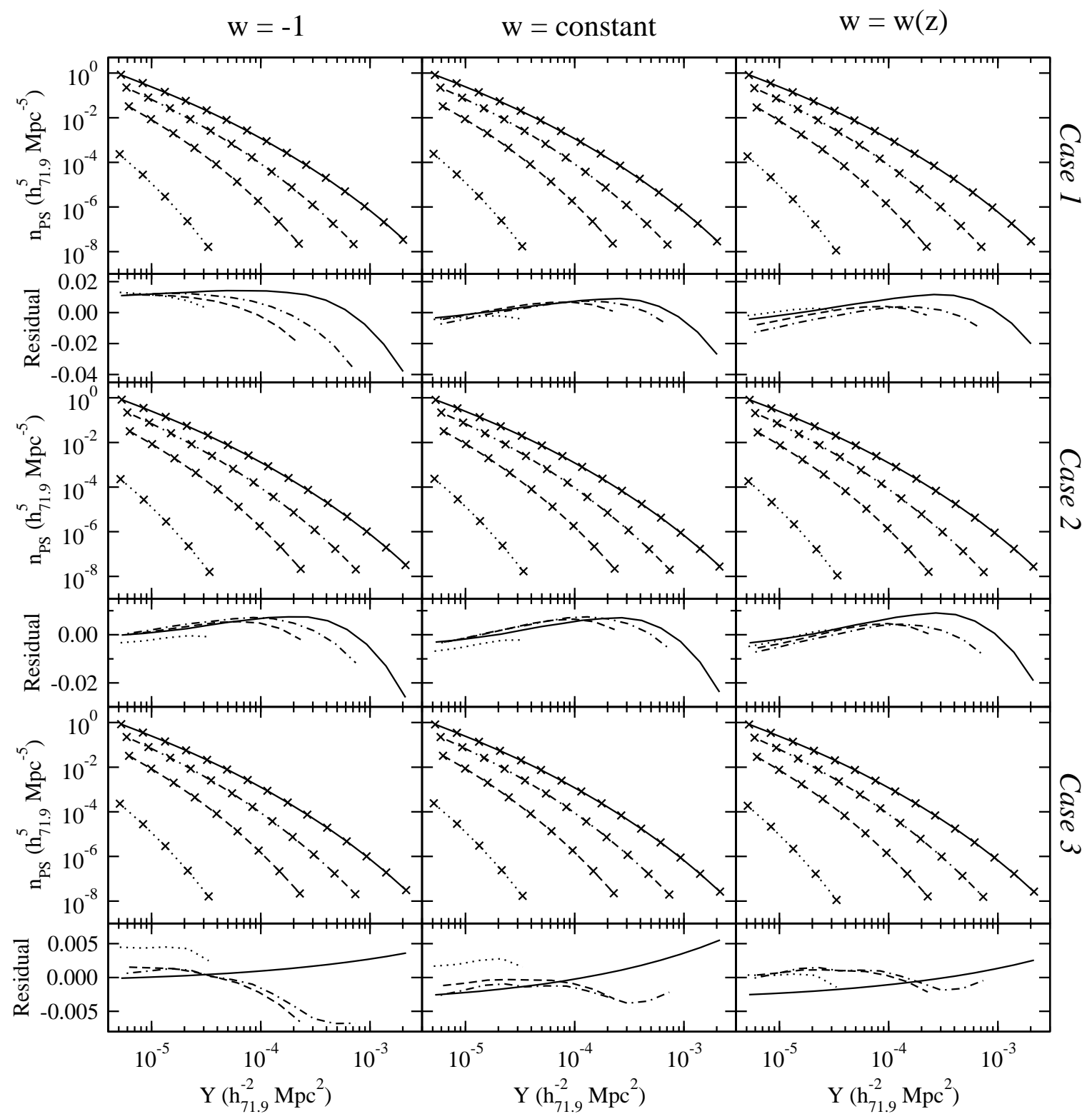

Fig. 4.3.- Non-equipartition $Y$ functions (crosses) and the best fitted $Y$ functions (lines) at different redshifts are plotted on each figure in row 1, 3, and 5 for Case 1, 2, and 3, respectively. Residuals in the $\log$ of the non-equipartition $Y$ functions are plotted under the corresponding figures. Figures in Column 1 correspond to models with dark energy parameters frozen at $w=1$. Figures in Column 2 correspond to models with $w$ fitted as constant parameters ( $w_{1}$ frozen at zero). Figures in Column 3 correspond to models with $w$ allowed to vary with redshift. Redshifts at $z=0,0.5,1$, and 2 are shown in solid, dash-dotted, dashed, and dotted lines, respectively. 


\section{Chapter 5}

\section{X-ray Signatures of}

\section{Non-Equilibrium Ionization Effects}

\section{in Galaxy Cluster Accretion Shock}

\section{Regions}

[Adapted from Wong, K.-W., \& Sarazin, C. L. 2010, ApJ, in preparation]

\subsection{Introduction}

Clusters of galaxies are very sensitive probes to cosmological parameters (e.g., Allen et al. 2008; Vikhlinin et al. 2009). Systematic uncertainties in precision cosmology using galaxy clusters can be minimized by restricting the sample of clusters to the highest degree of dynamical relaxation, and hence studying relaxed clusters is particularly important. In addition, the outer envelopes of clusters have been thought to be less subjected to complicated physics such as active galactic nucleus feedback, 
and hence these outer regions may provide better cosmological probes.

Clusters are believed to be formed by the continual merging and accretion of material from the surrounding large-scale structure. Based on high resolution $N$-body simulations, it has been found that cluster growth can be divided into an early fast accretion phase dominated by major mergers, and a late slow phase dominated by smooth accretion of background materials and many minor mergers (Wechsler et al. 2002; Zhao et al. 2009). Accretion shocks (or virial shocks) are unambiguous predictions of cosmological hydrodynamic simulations. For the most relaxed clusters with roughly spherical morphology in the outer regions, these simulations show that large amounts of material are accreted through filamentary structures in some particular directions, and more spherically symmetrically in other directions.

Most of the simulations assume the intracluster medium (ICM) is in collisional equilibrium. However, because of the very low density in the cluster outer regions ( $\gtrsim$ $R_{200}{ }^{1}$ ), the Coulomb collisional and collisional ionization timescales are comparable to the age of the cluster. It has been pointed out that electrons and ions there may be in non-equipartition (Fox \& Loeb 1997; Rudd \& Nagai 2009; Wong \& Sarazin 2009), and also the ionization state may not be in collisional ionization equilibrium (Yoshida et al. 2005). If these non-equilibrium processes are not properly taken into account, the measured properties may be biased in these regions.

Studying the outer regions of relaxed clusters not only is valuable in understanding the accretion physics and to test the assumptions concerning plasma physics near the shock regions, but it is also very important to test structure formation theory and to constrain the systematic uncertainties in clusters to be used as precision cosmological

\footnotetext{
${ }^{1} R_{\Delta}$ is the radius within which the mean total mass density of the cluster is $\Delta$ times the critical density of the universe. The virial radius $R_{\mathrm{vir}}$ is defined as a radius within which the cluster is virialized. For the Einstein-de Sitter universe, $R_{\mathrm{vir}} \approx R_{178}$, while for the standard $\Lambda$ CDM Universe, $R_{\mathrm{vir}} \approx R_{95}$.
} 
probes. Before the launch of the Suzaku X-ray observatory, physical properties such as temperature have never been constrained with confidence beyond roughly one-half of the shock radius. Most of our understanding of these regions is still based on theoretical models and hydrodynamic simulations. Recently, observations by Suzaku have constrained temperatures up to about half of the shock radius for a few clusters for the first time to better than a factor of $~ 2$ (George et al. 2009; Reiprich et al. 2009; Basu et al. 2010; Hoshino et al. 2010). While the uncertainties are still large, there is evidence that the electron pressure in cluster outer regions may be lower than that predicted by numerical simulations assuming collisional equilibrium. Observations of secondary cosmic microwave background anisotropies with the South Pole Telescope (SPT) and the Wilkinson Microwave Anisotropy Probe (WMAP) 7 year data also support these results (Lueker et al. 2009; Komatsu et al. 2010). These observational signatures are consistent with electrons and ions in non-equipartition, although it is also possible that the hydrodynamic simulations may simply overestimate the gas pressure. Another possibility is that heat conduction outside the cluster may be reducing the gas pressure (Loeb 2002). Recently, Wong et al. (2010) have shown that cosmological parameters will be biased if non-equilibrium effects (in particular, non-equipartition) are not properly taken into account.

More observations of the outer regions of clusters are being done or analyzed. In the future, the proposed International X-ray Observatory $(I X O)^{2}$ will have the sensitivity to constrain cluster properties out to the shock radius. Thus, a detailed study of the physics in the outer regions of clusters would be useful. In particular, $I X O$ will have sufficient spectral resolution to resolve many important X-ray lines, and hence the ionization state of the plasma can be determined.

In Wong \& Sarazin (2009), we have studied in detail the X-ray signature of non-

\footnotetext{
${ }^{2}$ http://ixo.gsfc.nasa.gov/
} 
equipartition effects in cluster accretion shock regions. In this chapter, we extend our study to include non-equilibrium ionization in our calculations. Non-equilibrium ionization calculations have been considered in a number of cosmological simulations to study the very low density warm-hot intergalactic medium (WHIM) surrounding galaxy clusters (Yoshida et al. 2005; Cen \& Fang 2006; Yoshikawa \& Sasaki 2006). At galaxy cluster scales, similar non-equilibrium ionization calculations have focused on merging clusters (Akahori \& Yoshikawa 2008, 2010). These studies generally agree that in the low density ICM and the WHIM, there are significant deviations from ionization equilibrium, and that the effects on the X-ray emission lines are strong. In this work, we focus on the X-ray signatures of non-equilibrium ionization in the accretion shock regions of relaxed clusters. We also discuss the detectability of nonequilibrium ionization effects with the future IXO.

The chapter is organized as follows. Section 5.2 describes in detail the physical models and techniques to calculate non-equilibrium ion fractions and X-ray observables, which includes the hydrodynamic models we used (Section 5.2.1), and the ionization and spectral calculational techniques (Sections 5.2.2-5.2.3). The overall dependence of the non-equilibrium ionization effects on the mass and redshift of the cluster is presented in Section 5.3. Calculated non-equilibrium ionization signatures are presented in Section 5.4, which includes descriptions of particular models we used to present the results (Section 5.4.1), X-ray spectra (Section 5.4.2), surface brightness profiles (Section 5.4.3), and the ratio of intensities of O VII and O VIII lines (Section 5.4.4). We discuss the detectability of O VII and O VIII lines and nonequilibrium ionization diagnostics with $I X O$ in Section 5.5. Section 5.6 gives the discussion and conclusions. Throughout the chapter, we assume a Hubble constant of $H_{0}=71.9 h_{71.9} \mathrm{~km} \mathrm{~s}^{-1} \mathrm{Mpc}^{-1}$ with $h_{71.9}=1$, a total matter density parameter 
of $\Omega_{M, 0}=0.258$, a dark energy density parameter of $\Omega_{\Lambda}=0.742$, and a cluster gas fraction of $f_{\text {gas }}=\Omega_{b} / \Omega_{M}=0.17$, where $\Omega_{b}$ is the baryon density parameter for our cluster models in the standard $\Lambda \mathrm{CDM}$ cosmology ${ }^{3}$. The clusters have a hydrogen mass fraction $X=76 \%$ for the ICM.

\subsection{Physical Models and Calculational Techniques}

\subsubsection{Hydrodynamic Models and Electron Temperature Structures}

We calculate the X-ray emission spectrum from the outer regions of cluster of galaxies using one-dimensional hydrodynamic simulations we have developed (Wong \& Sarazin 2009). The hydrodynamic models simulate the accretion of background material from the surrounding regions onto clusters through accretion shocks in the $\Lambda \mathrm{CDM}$ cosmology. The calculated hydrodynamic variables (e.g., density and temperature profiles) in the cluster outer regions are consistent with those calculated by threedimensional simulations, and a detailed discussion on our hydrodynamic simulations can be found in Wong \& Sarazin (2009).

The hydrodynamic simulations were done using Eulerian coordinates. In calculating the time-dependent ionization for the hydrodynamic models, we need to follow the Lagrangian history of each fluid element. In one-dimensional spherical symmetric systems, it is convenient to transform from the Eulerian coordinates to the Lagrangian coordinates by using the interior gas mass as the independent variable

$$
m_{g}(r)=4 \pi \int_{0}^{r} \rho_{g} r^{2} d r
$$

\footnotetext{
${ }^{3}$ http://lambda.gsfc.nasa.gov/product/map/dr3/parameters_summary.cfm
} 
where $r$ is the radius, and $\rho_{g}$ is the gas density. We determined the values of $m_{g}(r)$ for each of the grid zones in the hydrodynamical simulations in Wong \& Sarazin (2009) at the final redshift of $z=0$. The values of the density and temperature were also determined for this final time step for each gas element. Then, for each earlier time step, the values of gas density and temperature within the shock radius were determined by linear interpolation between the nearest two values of the interior gas mass on the grid at that time. When the values of $m_{g}(r)$ fall between the discontinuous values of the shocked and preshocked elements, we assume the materials to be preshock. This slightly underestimates the ionization timescale parameter defined below, but this effect is small as the grid zones in the simulations are closely spaced.

The collisional ionization and recombination rates and the excitation of X-ray emission depend on the free electron temperature $\left(T_{e}\right)$. Because the accretion shock may primarily heat ions instead of electrons due to the mass difference and the long Coulomb collisional timescale between electrons and ions, the electron temperature may not be the same as the ion temperature near the accretion shock regions (Fox \& Loeb 1997; Wong \& Sarazin 2009). The degree of non-equipartition depends on the non-adiabatic shock electron heating efficiency, which is defined as $\beta \equiv \Delta T_{e, \text { non-ad }} / \Delta \bar{T}_{\text {non-ad }}$ in Wong \& Sarazin (2009), where $\Delta T_{e, \text { non-ad }}$ and $\Delta \bar{T}_{\text {non-ad }}$ are the changes in electron temperature and average thermodynamic temperature due to non-adiabatic heating at the shock, respectively. While there are no observational constraints on $\beta$ in galaxy cluster accretion shocks, observations in supernova remnants suggest that $\beta \ll 1$ for shocks with Mach numbers similar to those in galaxy cluster accretion shocks (Ghavamian et al. 2007). Wong \& Sarazin (2009) have calculated electron temperature profiles for models with a very low electron heating efficiency $(\beta=1 / 1800)$, an intermediate electron heating efficiency $(\beta=0.5)$, and an 
equipartition model $(\beta=1)$. The electron temperature profiles of these models with different values of $\beta$ are used to calculate the ionization fractions and X-ray emission in this chapter.

The ionization timescale parameter of each fluid element is defined as

$$
\tau=\int_{t_{s}}^{t_{0}} n_{e} d t
$$

where $n_{e}$ is the electron number density, $t_{s}$ is the time when the fluid element was shocked, and $t_{0}$ is the time at the observed redshift. The ionization timescale parameters for cluster models with accreted masses of $M_{\mathrm{sh}}=0.77,1.53$, and $3.06 \times 10^{15} M_{\odot}$ are shown in Figure 5.1. Most ions of astrophysical interest will not achieve ionization equilibrium for the ionization timescale parameters $\lesssim 10^{12} \mathrm{~cm}^{-3} \mathrm{~s}$ (Smith \& Hughes 2010).

\subsubsection{Ionization Calculations}

In order to calculate the X-ray emission spectrum for non-equilibrium ionization plasma, the ionization state of each fluid element has to be calculated. We assume $\mathrm{H}$ and He are fully ionized.

In this chapter, we consider only collisional ionization processes, and ignore photoionization. To justify this, we compare the photoionization and collisional ionization rate for the ions we are interested in. The photoionization rate of an ion $i$ is given by

$$
R_{\mathrm{PI}}=\int_{0}^{\infty} \frac{\sigma_{i}(\nu) F(\nu)}{h \nu} d \nu
$$

where $\sigma_{i}(\nu)$ is the photoionization cross section of ion $i$ at frequency $\nu$, and $F(\nu)$ is the ionizing flux. Near the cluster accretion shock, the dominant photoionization 
source is the UV background. We approximate the UV ionizing flux at $z=0$ as a power law $F(\nu)=F_{0}\left(\nu / \nu_{0}\right)^{-\Gamma}$ with $F_{0}=10^{22} \mathrm{erg} \mathrm{cm}^{-2} \mathrm{~s}^{-1} \mathrm{~Hz}, \nu_{0}=10^{15.5} \mathrm{~Hz}$, and $\Gamma=1.3$ (Haardt \& Madau 1996). For example, for the O VII and O VIII ions we are most interested in throughout this chapter, adopting the photoionization cross sections given by Verner et al. (1996) gives $R_{\mathrm{PI}}(\mathrm{O}$ VII $)=5.2 \times 10^{-18} \mathrm{~s}^{-1}$ and $R_{\mathrm{PI}}(\mathrm{O}$ VIII $)=1.7 \times 10^{-18} \mathrm{~s}^{-1}$. The slowest collisional ionization rate of the oxygen ions is of the order of $10^{-16}\left(n_{e} / 10^{-5} \mathrm{~cm}^{-3}\right) \mathrm{s}^{-1}$ for typical temperatures $(>1 \mathrm{keV})$ and densities in the outer regions of clusters (Smith \& Hughes 2010). This is nearly two orders of magnitude higher than the photoionization rates of O VII and O VIII ions. Even at $z=3$ when the UV background is roughly 80 times stronger (Haardt \& Madau 1996), the densities in the outer regions of clusters will be higher by a factor of $\sim(1+z)^{3}=64$, and hence collisional ionization rates will increase by a similar factor. Collisional ionization still dominates over photoionization. For heavier elements, the photoionization rates will be even smaller, but the collisional ionization rates for ions up to those of $\mathrm{Ni}$ are all higher than $\sim 10^{-17}\left(n_{e} / 10^{-5} \mathrm{~cm}^{-3}\right) \mathrm{s}^{-1}$ (Smith \& Hughes 2010). Therefore, we assume that photoionization is not important in our calculations.

For ionization dominated by collisional processes at low densities, the ionization states for each of the ions of a given element $X$ are governed by

$$
\frac{d f_{i}}{d t}=n_{e}\left\{C_{i-1}\left(T_{e}\right) f_{i-1}+\alpha_{i}\left(T_{e}\right) f_{i+1}-\left[C_{i}\left(T_{e}\right)+\alpha_{i-1}\left(T_{e}\right)\right] f_{i}\right\}
$$

where $f_{i} \equiv n\left(X^{+i-1}\right) / n(X)$ is the ionization fraction of the ion $i$ with charge $+i-1$, $n\left(X^{+i-1}\right)$ is the number density of that ion, $n(X)$ is the total number density of the element $X$, and $C_{i}\left(T_{e}\right)$ and $\alpha_{i}\left(T_{e}\right)$ are the coefficients of collisional ionization out of and recombination into the ion $i$, respectively. In solving equation (5.4), we use 
an eigenfunction technique which is based on the algorithm developed by Hughes \& Helfand (1985), and the method is described in detail in Appendix A of Borkowski et al. (1994). The eleven heavy elements C, N, O, Ne, Mg, Si, S, Ar, Ca, Fe, and Ni are included in our calculations. The eigenvalues and eigenvectors, which are related to the ionization and recombination rates, used to solve equation (5.4) are taken from the latest version of the nei version 2 model in $\mathrm{XSPEC}^{4}$ (version 12.6.0). The atomic physics used to calculated the eigenvalues and eigenvectors in XSPEC are discussed in Borkowski et al. (2001), and the ionization fractions in the latest version of XSPEC are calculated using the updated dielectronic recombination rates from Mazzitelli et al. (1998). All of the eleven heavy elements are assumed to be neutral initially when solving equation (5.4).

\subsubsection{X-ray Emission Calculations}

Once the ionization states are calculated by solving equation (5.4), the X-ray emission spectrum could be calculated by using available plasma emission codes such as the Raymond-Smith code (Raymond \& Smith 1977), the SPEX code (Kaastra et al. 1996), and the APEC code (Smith et al. 2001). We chose to use a version of the APEC code as implemented in the nei version 2 model in XSPEC to calculate the X-ray emission spectrum. The routine uses the Astrophysical Plasma Emission Database ${ }^{5}$ (APED) to calculate the resulting spectrum. Thus, the atomic data we used to calculate the ionization fractions and spectrum are mutually consistent, both coming from the same nei model version 2.0 in XSPEC.

We assume the abundance to be 0.3 of solar for all models, and use the solar abundance tables of Anders \& Grevesse (1989).

\footnotetext{
${ }^{4}$ http://heasarc.nasa.gov/xanadu/xspec/

${ }^{5}$ http://cxc.harvard.edu/atomdb/
} 
We calculated the X-ray emissivity in photons per unit time per unit volume per unit energy, $\epsilon_{E}$, for each fluid element in our hydrodynamic models at redshift zero, which can be expressed in terms of an emissivity function, $\Lambda_{E}$, by

$$
\epsilon_{E}=\Lambda_{E} n_{e} n_{p}
$$

where $n_{p}$ is the proton density. The emissivity function $\Lambda_{E}$ depends on the ionization fractions and the electron temperature, but is independent of the gas density. The projected spectrum in the rest frame is given by integrating the X-ray emissivity along the line of sight

$$
I_{E}=\int \epsilon_{E} d l
$$

where $l$ is the distance along the line of sight. The broadband rest frame surface brightness in energy per unit time per unit area is then given by

$$
S_{E}=\int I_{E} E d E
$$

where the integral is across the energy band of interest.

\subsection{Dependence of Non-Equilibrium Ionization on Cluster Mass and Redshift}

We consider how the degree on non-equilibrium ionization of a cluster depends on its mass and redshift. We first consider how the cluster parameters which affect the ionization depend on mass and redshift. In order to compare equivalent locations in the different clusters, we estimate the ionization parameters at a given over-density 
radius $R_{\Delta}$. We consider a simple self-similar scaling argument for galaxy clusters.

By definition, the average total density within the radius $R_{\Delta}$ is

$$
\rho_{\Delta} \equiv \Delta \rho_{\text {crit }}(z)=\frac{3 \Delta}{8 \pi G} H^{2}(z)=\frac{3 \Delta H_{0}^{2}}{8 \pi G} E^{2}(z)
$$

where $\rho_{\text {crit }}(z)$ is the critical density at redshift $z$, and $H(z)$ is the Hubble constant at redshift $z$. The quantity $E(z)=H(z) / H_{0}$, so that

$$
\begin{aligned}
E^{2}(z) & =\left[\Omega_{M}(1+z)^{3}+\Omega_{R}(1+z)^{4}+\Omega_{\Lambda}+\left(1-\Omega_{M}-\Omega_{R}-\Omega_{\Lambda}\right)(1+z)^{2}\right] \\
& \approx \Omega_{M}(1+z)^{3}+1-\Omega_{M}
\end{aligned}
$$

The final expression in equation (5.9) follows from the assumption that the universe is flat $\left(\Omega_{M}+\Omega_{R}+\Omega_{\Lambda}=1\right)$ and the fact that the radiation density parameter is small $\left(\Omega_{R} \ll 1\right)$ in the present-day universe. The gas density at $R_{\Delta}$ is roughly

$$
\rho_{\text {gas }}\left(R_{\Delta}\right) \approx f_{\text {gas }} \rho_{\Delta} \sim E^{2}(z)
$$

The inflow timescale is roughly

$$
t \approx\left(G \rho_{\Delta}\right)^{-1 / 2} \approx t_{H}(0)[E(z)]^{-1}
$$

where $t_{H}(0) \equiv 1 / H_{0}$ is the Hubble time at $z=0$. Thus, the ionization timescale parameter $\tau$ varies as

$$
\tau \sim E(z)
$$

Thus, the value of $\tau$ at a fixed characteristic radius should be nearly independent of the cluster mass but will increase significantly with redshift. Figure 5.1 shows that 
the variation of $\tau$ with radius is fairly self-similar for clusters with differing masses. When these curves are scaled to a fixed cluster characteristic radius, they are very nearly identical.

The ionization state of the gas depends on $\tau$ and the collisional ionization and recombination rates. For an under-ionized plasma, the collisional ionization rates are more important, so that the ionization state should depend mainly on $C_{i}\left(T_{e}\right) \tau$. For an under-ionized plasma where the electron temperature is greater than the ionization potential of the relevant ions, $C_{i}\left(T_{e}\right)$ varies slowly, $C_{i}\left(T_{e}\right) \sim T_{e}^{1 / 2}$. We have confirmed that this dependence fits the temperature dependence of the ionization rate of O VII over the interesting temperature range $\left(k T_{e}=1-5 \mathrm{keV}\right)$. Below, we show that the ratio of $\mathrm{O}$ VIII to $\mathrm{O}$ VII lines is the best diagnostic of departures from ionization equilibrium (Section 5.4.4).

We assume that the electron temperature increases in proportion to the mean temperature $T_{\Delta}$ at the radius $R_{\Delta}$. The mass within $R_{\Delta}$ is $M_{\Delta}=(4 \pi / 3) R_{\Delta}^{3} \rho_{\Delta}$, so that the radius $R_{\Delta}$ is given by

$$
R_{\Delta}=\left(\frac{2 G}{H_{0}^{2} \Delta}\right)^{1 / 3} M_{\Delta}^{1 / 3}[E(z)]^{-2 / 3}
$$

The condition of hydrostatic equilibrium or the shock jump condition at the accretion shock imply that $k T_{\Delta} \approx G M_{\Delta} / R_{\Delta}$. Thus, the gas temperature varies as

$$
T_{\Delta} \sim M_{\Delta}^{2 / 3}[E(z)]^{2 / 3}
$$

This implies that

$$
C_{i}\left(T_{e}\right) \sim M_{\Delta}^{1 / 3}[E(z)]^{1 / 3}
$$


Combining equations (5.12) and (5.15) gives

$$
C_{i}\left(T_{e}\right) \tau \sim M_{\Delta}^{1 / 3}[E(z)]^{4 / 3} .
$$

Equations (5.12) and (5.16) suggest that the ionization state should depend only very weakly on the cluster mass, but should depend strongly on cluster redshift. The increase of $\tau$ and $C_{i}\left(T_{e}\right) \tau$ with $z$ implies that non-equilibrium ionization will be most important in low redshift clusters.

Later, we will show that the ratio of O VIII to O VII lines is the best diagnostic of departures from ionization equilibrium (Section 5.4.4). Figure 5.2 shows the dimensionless ionization parameter $C_{i}\left(T_{e}\right) \tau$ for $\mathrm{O}$ VII as a function of the scaled radius $\left(r / R_{\mathrm{sh}}\right)$ for clusters with different masses at $z=0$. Here, $R_{\mathrm{sh}}$ is the radius of the cluster accretion shock. Models with $\beta=1 / 1800$ (non-equipartition) and $\beta=1$ (equipartition) are shown in thick and thin lines, respectively. All the curves for the same $\beta$ but with different masses nearly overlap each others. This confirms that the non-equilibrium ionization effect at the same characteristic radius is nearly independent of mass.

Figure 5.3 shows the dimensionless ionization parameter as a function of the scaled radius for cluster models at different redshifts. The cluster model with a total accreted mass $M_{\mathrm{sh}}=1.53 \times 10^{15} M_{\odot}$ at $z=0$ is used. Models with $\beta=1 / 1800$ (non-equipartition) and $\beta=1$ (equipartition) are shown in thick and thin lines, respectively. In contrast to the dependence in mass, we find that there is significant evolution with redshift. The non-equilibrium ionization effect should be strongest for low-redshift clusters.

The preceding arguments assumed that departures from ionization equilibrium could be assessed through the variation of $C_{i}\left(T_{e}\right) \tau$. To test this explicitly and to 
determine if the preceding arguments apply to spectral diagnostics for non-equilibrium ionization, we study the effects of non-equilibrium ionization on O VII and O VIII ion fractions using numerical simulations. We calculate the non-equilibrium ionization bias, $b$, of the ionization fraction ratios $f(\mathrm{O}$ VIII $) / f(\mathrm{O}$ VII $)$ for the NEI and CIE models with the same electron heating efficiency $\beta$. The non-equilibrium ionization bias is defined as

$$
b \equiv \frac{[f(\mathrm{O} \text { VIII }) / f(\mathrm{O} \text { VII })]_{\mathrm{NEI}}}{[f(\mathrm{O} \text { VIII }) / f(\mathrm{O} \text { VII })]_{\mathrm{CIE}}} .
$$

Figure 5.4 shows the non-equilibrium ionization bias as a function of the scaled radius for clusters with different masses. Models with $\beta=1 / 1800$ (non-equipartition) and $\beta=1$ (equipartition) are shown in thick and thin lines, respectively. The nearly selfsimilar curves justify the semi-analytical argument that non-equilibrium ionization effect at the same characteristic radius is nearly independent of mass. Note that non-equilibrium ionization effect on the $f(\mathrm{O}$ VIII $) / f(\mathrm{O}$ VII $)$ ratio is only significant for the outer $10 \%$ of the shock radius. However, the projected O VII and O VIII line emission will be significantly affected even at a radius as small as one-fourth of the shock radius. This is because the projected emission at the inner radius can be dominated by the line emission from the under-ionized outer shell (Section 5.4.4 below).

Figure 5.5 shows the non-equilibrium ionization bias as a function of the scaled radius for cluster models at different redshifts. The cluster model with a total accreted mass $M_{\mathrm{sh}}=1.53 \times 10^{15} M_{\odot}$ at $z=0$ is used. Models with $\beta=1 / 1800$ (non-equipartition) and $\beta=1$ (equipartition) are shown in thick and thin lines, respectively. The non-equilibrium ionization effect is larger for clusters at lower redshifts which agrees with the semi-analytical argument given above. Note that at a redshift higher than 1 , only a thin shell with a width of less than $5 \%$ of the shock 
radius is in non-equilibrium ionization compared to the wider shell with a width of $\sim 10 \%$ of the shock radius for zero redshift clusters.

\subsection{Non-Equilibrium Ionization Signatures}

\subsubsection{Models Used to Calculate Spectra}

Massive clusters at low redshifts are ideal candidates to study the non-equilibrium ionization effects in the outer regions since the departures from ionization equilibrium are larger at low redshift and nearly independent of cluster mass (Section 5.3). Clusters with higher masses are more luminous in X-rays, and hence the spectral signatures are easier to detect. In the following, we present X-ray spectra for the hydrodynamic cluster model with an accreted mass of $M_{\mathrm{sh}}=1.53 \times 10^{15} M_{\odot}$ at $z=0$ calculated in Wong \& Sarazin (2009). This model represents a typical massive cluster in the present-day universe. The shock radius is $R_{\mathrm{sh}}=4.22 \mathrm{Mpc}$ for this model. The virial radius is $R_{\mathrm{vir}}=R_{95}=2.75 \mathrm{Mpc}$, and the total mass within $R_{95}$ is $M_{95}=1.19 \times 10^{15} M_{\odot}$. Another commonly used radius and mass are $R_{200}=1.99 \mathrm{Mpc}$ and $M_{200}=9.50 \times 10^{14} M_{\odot}$.

We calculate spectra for three different values of the shock electron heating efficiency $\beta=1 / 1800,0.5$, and 1 . The last case corresponds to electron-ion equipartition. We also calculate spectra both for non-equilibrium ionization (NEI) models and for collisional ionization equilibrium (CIE) models for comparison. For the NEI models, the results for the model with the small shock electron heating efficiency $\beta=1 / 1800$ will be discussed extensively throughout the chapter, as this model maximizes the departures from equilibrium in the outer regions of clusters. For the CIE models, we present a non-equipartition model with $\beta=1 / 1800$ (CIE-Non-Eq) and an equiparti- 
tion model with $\beta=1$ (CIE-Eq).

\subsubsection{X-ray Spectra}

The projected rest frame spectra for several different models at two projected radii are shown in Figure 5.6. In the upper panels, we show the NEI model with a very small shock heating efficiency $(\beta=1 / 1800)$, and electrons and ions are in non-equipartition. The CIE-Non-Eq model is shown in the lower panels. The left panels show spectra at a radius of $r=2 \mathrm{Mpc}$, while the right panels show spectra at a radius of $r=3.5 \mathrm{Mpc}$. Each spectrum is binned with a bin size of $\Delta \log (E)=0.005$.

At $r=2 \mathrm{Mpc}$, the overall spectra are dominated by the free-free continuum emission over a wide range of energy for both the NEI and the CIE-Non-Eq models. The continuum spectra for both models are nearly identical because of the dominant free-free emission with the same electron temperature. The line emission is also very similar for both models because at this radius, the ionization timescale parameter is rather large $\left(\tau \sim 8 \times 10^{12} \mathrm{~cm}^{-3} \mathrm{~s}\right)$. The most notable difference is the line intensity of the $\mathrm{O}$ VII triplet lines near $\sim 0.57 \mathrm{eV}$. This line intensity for the NEI model is much higher than that of the CIE-Non-Eq model. There is almost no O VII line emission for the CIE-Non-Eq model. The strong O VII at $r=2 \mathrm{Mpc}$ for the NEI model is mainly due to the projection of emission from the under-ionized O VII ions in the outer regions with much shorter ionization timescale parameters. The differences for other strong emission lines are much smaller between the two models. There is slightly more soft line emission below about $1 \mathrm{keV}$ for the NEI model.

At $r=3.5 \mathrm{Mpc}$, there are significant differences in the spectra between the NEI and the CIE-Non-Eq models. For the CIE-Non-Eq model, the continuum emission still dominates the overall spectrum; for the NEI model, the soft emission is dominated 
by lines. One of the most obvious differences in the line emission between the two models is again for the O VII triplet. The line intensity for the NEI model is much higher than that of the CIE-Non-Eq model. The O VII triplet is weak for the CIENon-Eq model. By inspecting a number of line ratios at different radii, we found that the ratio of the $\mathrm{O}$ VII and $\mathrm{O}$ VIII line intensities can be used as a diagnostic for the degree of ionization equilibrium, and this will be discussed in Section 5.4.4 below.

\subsubsection{Surface Brightness Profiles}

The rest frame radial surface brightness profiles integrated over various energy bands for the outer regions of clusters are shown in Figure 5.7. The NEI models with $\beta=1 / 1800$ are shown in thick lines in both the upper left and upper right panels. The CIE-Non-Eq and the CIE-Eq models are shown as thin lines on the upper left and upper right panels, respectively. The ratios of $S_{\mathrm{NEI}} / S_{\mathrm{CIE}-\mathrm{Non}-\mathrm{Eq}}$ and $S_{\mathrm{NEI}} / S_{\mathrm{CIE}-\mathrm{Eq}}$ are shown below the corresponding panels. Comparing the NEI and the CIE-Non-Eq models tells us the effects of non-equilibrium ionization alone, while comparing the NEI and the CIE-Eq model tells us the total effects of both non-equilibrium ionization and non-equipartition.

From the left panels of Figure 5.7, we can see that non-equilibrium ionization significantly enhances the soft $(0.3-1.0 \mathrm{keV})$ emission in the outer regions. For the NEI model, the soft emission has been increased by more than $20 \%$ at around $3 \mathrm{Mpc}$, and up to nearly an order of magnitude around the shock radius compared to the CIE-Non-Eq model. The increase of the soft emission is due to the line emission by the under-ionized ions. For the CIE-Non-Eq model, the surface brightness profiles in all energy bands shown in Figure 5.7 decrease rapidly out to the shock radius. For the NEI model, the decrease in surface brightness in the soft band as a function 
of radius slows down near $\sim 3 \mathrm{Mpc}$, and the soft band surface brightness actually increases with radius from $\sim 3.7 \mathrm{Mpc}$ out to nearly the shock radius, where the surface brightness drops rapidly. Within about $2.3 \mathrm{Mpc}$, non-equilibrium ionization effect is less than $5 \%$ in the soft band. The non-equilibrium ionization effect on the medium $(1.0-2.0 \mathrm{keV})$ band is not as dramatic as the soft band, and the maximum increase is about $70 \%$ near $3.8 \mathrm{Mpc}$. The non-equilibrium ionization effect on the hard (2.0$10.0 \mathrm{keV}$ ) band is less than $5 \%$ in most regions, and the effect is to lower the hard emission near the shock radius. The soft emission dominates the overall X-ray band (0.3-10.0 keV) outside of $\sim 3 \mathrm{Mpc}$, and the overall X-ray emission decreases more slowly than for the CIE-Non-Eq model beyond that radius. Near the shock radius, the surface brightness in the overall X-ray band for the NEI model is about a fact of 6 higher than that for the CIE-Non-Eq model.

The right panels of Figure 5.7 show that in addition to the non-equilibrium ionization effect, non-equipartition will increase the soft emission by a significant factor near the shock radius. This occurs because in the CIE-Non-Eq and NEI models, the electron temperatures in the outer regions are lower than for the CIE-Eq model, and this also leads to more soft X-ray line emission. The surface brightness profile in the overall X-ray band for the NEI model is a factor of 5 higher than that for the CIE-Eq model near the shock radius. A detailed discussion on the difference between the CIE-Non-Eq and CIE-Eq models can be found in Wong \& Sarazin (2009).

\subsubsection{O VII and O VIII Line Ratio}

The most prominent non-equilibrium ionization signature in the X-ray lines are for the line ratio of O VII and O VIII. Figure 5.8 shows the spectra for models at $r=2 \mathrm{Mpc}$. The spectra are shown in the $0.5-0.7 \mathrm{keV}$ range which covers the rest frame energies 
of the O VII and O VIII lines. The upper panel shows the NEI model with a very low electron heating efficiency $\beta=1 / 1800$. The middle panel shows the NEI model with an intermediate $\beta=0.5$. The lower panel shows the CIE-Non-Eq model with $\beta=1 / 1800$. Each spectrum is binned with a bin size of $\Delta E=0.0001 \mathrm{keV}$.

In the upper panel of Figure 5.8, the most prominent lines are the He-like O VII triplets at 561.0, 569.6, and $574.0 \mathrm{eV}$, the H-like $\mathrm{O}$ VIII doublets at 653.5 and $653.7 \mathrm{keV}$, and the He-like O VII line at $665.6 \mathrm{eV}$. Here, we focus on the line ratio between the He-like O VII triplets and the H-like O VIII doublets as a diagnostic for non-equilibrium ionization. These two lines have been used to search for the WHIM as well as to study the non-equilibrium ionization of the WHIM (Cen \& Fang 2006; Yoshikawa \& Sasaki 2006). We find that the He-like O VII and the H-like O VIII lines also show strong signatures of non-equilibrium ionization in the outer regions of clusters.

The upper and lower panels of Figure 5.8 show that non-equilibrium ionization strongly enhances the He-like O VII triplets compared to the CIE-Non-Eq model. The H-like O VIII doublets for the NEI model are only slightly stronger than for the CIE-Non-Eq model. This suggests that the ratio of the O VII and O VIII lines can be used as a diagnostic for non-equilibrium ionization. The O VII and O VIII lines are similarly strong for the NEI models with electron heating efficiencies $\beta=0.5$ and $1 / 1800$, and we suggest that the O VII and O VIII line ratio is a good diagnostic for the non-equilibrium ionization for a wide range of electron heating efficiencies.

Figure 5.9 shows the surface brightness for the O VII and O VIII lines for the NEI model with $\beta=1 / 1800$. The surface brightness of the lines are calculated by subtracting the continuum surface brightness from the total surface brightness within narrow energy ranges of $556.0-579.0 \mathrm{eV}$ and $648.5-658.7 \mathrm{eV}$ for the O VII triplets and 
the O VIII doublets, respectively. The energy bands were chosen to cover the O VII and the O VIII lines with a spectral resolution of $10 \mathrm{eV}$ which is the expected value for the outer arrays of the X-ray Microcalorimeter Spectrometer (XMS) on the IXO. The continuum surface brightness were calculated by fitting the spectrum to a power law model in the energy range of $0.5-0.7 \mathrm{keV}$, excluding the lines. This simulates the techniques likely to be used to analyze real observations. We also show the continuum surface brightness within the narrow energy bands used to extract the line surface brightness in Figure 5.9.

The surface brightness of the O VII triplets is nearly constant from $\sim 1 \mathrm{Mpc}$ to $\sim 3 \mathrm{Mpc}$, and then rises gradually. Note that a flat surface brightness at inner radii and rising surface brightness at larger radii is the signature of a shell of emission at large radii seen in projection. That is, most of the O VII emission is actually at large radii where the ionization time scale is short. Beyond about $3.9 \mathrm{Mpc}$, the O VII surface brightness rises rapidly to a peak value, and then drops. The continuum in the 556.0-579.0 eV energy band drops from a radius of $1 \mathrm{Mpc}$ out to the shock radius. Beyond $\sim 2.8 \mathrm{Mpc}$, the $\mathrm{O}$ VII line emission dominates over the continuum in the 556.0-579.0 eV energy band. For the O VIII doublets, the surface brightness drops from a radius of $1 \mathrm{Mpc}$ out to $\sim 3 \mathrm{Mpc}$, and then rises to a peak value at $\sim 4 \mathrm{Mpc}$. The O VIII surface brightness then drops beyond $\sim 4$ Mpc. The O VIII line emission dominates over the continuum in the 648.5-658.7 eV energy band beyond $\sim 2 \mathrm{Mpc}$.

Figure 5.10 shows the line ratios of O VII and O VIII, $S(\mathrm{O}$ VIII)/S(O VII), for different models. To compare the effect of non-equilibrium ionization alone, we can compare the line ratios between the NEI model (solid line) and the CIE-Non-Eq model (dashed line), while both models assume an electron heating efficiency $\beta=1 / 1800$. Both models use the same non-equipartition electron temperature to calculate the 
spectra, but one of them assumes equilibrium ionization while the other one does not. At $\sim 1 \mathrm{Mpc}$, the line ratio for the NEI model is more than a factor of 2 lower than that of the CIE-Non-Eq model. The difference increases as the radius increases, and the differences are over an order of magnitude for radii beyond $\sim 2 \mathrm{Mpc}$. For the CIE-Eq model (dotted line), the line ratio is very similar to that of the CIENon-Eq model, except near the shock regions where the line ratio for the CIE-Eq model is a factor of a few higher than the CIE-Non-Eq model. Both the line ratios of the CIE-Eq and the CIE-Non-Eq models are above 10 in most regions between 14 Mpc. NEI models with electron heating efficiencies $\beta=0.5$ and 1.0 are also shown in Figure 5.10. The effect of increasing the electron heating efficiency is to raise the electron temperature at the shock, and hence increase the ionization rates. From Figure 5.10, we can see that increasing the electron heating efficiency only affects the line ratio by less than a factor of two in most regions shown compared to the NEI model with $\beta=1 / 1800$. The line ratios for all the NEI models with different electron heating efficiencies are less than 10 for radii beyond about $1.3 \mathrm{Mpc}$. In summary, the line ratios in the outer regions for all the NEI models we calculated are significantly smaller than those for the CIE models, and the differences are larger than an order of magnitude for most regions beyond $\sim 2$ Mpc. Such large differences can be used to distinguish between NEI and CEI models in real observations. 


\subsection{Detectability of $\mathrm{O}$ VII and $\mathrm{O}$ VIII Lines with $I X O$ and Testing the Non-Equilibrium Ioniza- tion Effect}

In this section, we estimate whether the O VII and O VIII lines in cluster outer regions and the non-equilibrium ionization signatures can be detected with $I X O$. We consider the NEI model with $\beta=1 / 1800$ for a cluster with an accreted mass of $M_{\mathrm{sh}}=1.53 \times 10^{15} M_{\odot}$ at low redshift. The choice is justified by the fact that the non-equilibrium ionization effect does not depend strongly on mass and the effect is larger at lower redshift (Section 5.3), and that the surface brightness for massive and low redshift clusters is higher.

The X-ray Microcalorimeter Spectrometer (XMS) planned for the IXO can potentially detect the O VII and O VIII lines in cluster outer regions. The Wide Field Imager does not have enough spectral resolution $(>50 \mathrm{eV})$ at around $0.6 \mathrm{keV}$, and the O VII triplets cannot be resolved from the $0.5 \mathrm{keV}$ nitrogen line. The X-ray Grating Spectrometer covers the interesting energy range, but the collecting area is too small to detect the weak O VII and O VIII lines. Therefore, we only consider the XMS in our estimations. The XMS has inner (core) and outer microcalorimeter arrays with expected spectral resolutions of 2.5 and $10 \mathrm{eV}$, respectively. We consider two cases when observing with the XMS. The first case (XMSC) is that only the inner core array is used, and the second case (XMSF) is that the full array (both inner and outer arrays) is used. For simplicity, when considering the XMSF, we assume the spectral resolution to be the same as the outer array throughout the full array. This doesn't strongly affect the detectability of the lines, since the bands used to determine the fluxes in the lines are set by the line width in the outer array, and the line width in 
the inner core doesn't affect the line flux. The expected effective areas $\left(A_{\text {eff }}\right)$ for the XMS is about $10000 \mathrm{~cm}^{2}$ at around $0.6 \mathrm{keV}$. The relevant instrument parameters for the two cases we considered are listed in Table 5.1.

\subsubsection{Backgrounds}

The major background for the IXO observations are Non-X-ray Background (NXB), the soft emission from the local Galactic background (GXB), and these are included in our signal-to-noise ratio calculations. The cluster continuum emission will be much weaker than the line emission in the band widths we are interested in, but we also include the cluster continuum emission in our calculations. With the spatial resolution of $5^{\prime \prime}$ and the very long exposure time needed for the line detection, point source contaminations will be negligible, and hence it is not included in our calculations. In fact, the GXB we used has included a component from unresolved AGNs, and this may overestimate the total background. The total count rates of the backgrounds we used for the two instrument setups are listed in Table 5.2, and are discussed below.

We use the GXB simulated by Fang et al. (2005) which included two thermal components to represent the Local Hot Bubble emission and the transabsorption emission (Snowden 1998; Kuntz \& Snowden 2000), and one continuum emission component to represent unresolved AGN background. The parameters used for their background model are based on McCammon et al. (2002). The most prominent emission around $0.6 \mathrm{keV}$ is the line emission from nitrogen and oxygen ions (Figure 6 in Fang et al. 2005). With the very high XMS spectral resolutions, the O VII and O VIII lines from clusters beyond $z \approx 0.028$ should be separated from the strong line emission in the GXB (see Table 5.3 below). We adopt the continuum intensity value of $I_{\mathrm{GXB}}=24$ photons $\mathrm{cm}^{-2} \mathrm{~s}^{-1} \mathrm{sr}^{-1} \mathrm{keV}^{-1}$ at around $0.6 \mathrm{keV}$, which is used in Fang et al. (2005). 
The total count rate for the whole field of view $\left(\Omega_{\mathrm{FOV}}\right)$ which covers the energy range of the lines is given by $R_{\mathrm{GXB}}=I_{\mathrm{GXB}} \times A_{\mathrm{eff}} \times \Omega_{\mathrm{FOV}} \times \Delta E_{\mathrm{band}}$, where $\Delta E_{\mathrm{band}}$ is the bandwidth covered the lines. For the O VIII doublets, since the line separation is much smaller than the spectral resolutions of all the instruments we considered, $\Delta E_{\mathrm{band}}$ is simply the spectral resolution of the corresponding instrument. For the O VII triplets, the three lines should be well separated by the XMS core array. Hence, $\Delta E_{\text {band }}$ of O VII is then three times the spectral resolution of the XMS core array. However, for the XMS full array, the O VII triplets cannot be resolved. Therefore, $\Delta E_{\text {band }}$ is then given by the maximum separation of the triplets $(13 \mathrm{eV})$ plus the corresponding spectral resolutions.

For a future mission like the $I X O$, the NXB is rather uncertain. We use the count rate of $F_{\mathrm{NXB}}=8.1 \times 10^{-3}$ photons $\mathrm{s}^{-1} \operatorname{arcmin}^{-2} \mathrm{keV}^{-1}$ at $0.6 \mathrm{keV}$ for the XMS estimated by the IXO team (Smith et al. 2010). The total count rate for the whole field of view which covers the energy range of the lines is given by $R_{\mathrm{NXB}}=$ $F_{\mathrm{NXB}} \times \Omega_{\mathrm{FOV}} \times \Delta E_{\mathrm{band}}$. To address the effects of the uncertainties, we also multiply the NXB by factors of 0.5 and 2 in our calculations (Section 5.5.2 below).

The cluster continuum emission within the very narrow energy bands we are interested in are much weaker than the line emission in cluster outer regions (Figure 5.9) and the GXB. To detect the O VII and O VIII lines, it is also important to observe regions where the cluster continuum emission is weak compared to the line emission. Therefore, the cluster continuum emission should not be important when estimating the signal-to-noise ratio. Nevertheless, we have included the cluster continuum emission in our calculations. To be conservative, we simply take the cluster continuum emission at $r=2.8 \mathrm{Mpc}$ where the cluster continuum emission across $648.5-658.7 \mathrm{eV}$ (energy range where the O VII triplets are covered by the XMS full array) equal to 
the O VII emission. The continuum count rates $R_{\text {cont }}$ are listed in Table 5.2.

\subsubsection{Signal-to-Noise Ratio}

In order for the lines to be separated from the local GXB, clusters to be observed should be at high enough redshifts. Since the O VII triplets spread across $13 \mathrm{eV}$, the best targets should have redshifts such that the lines can be shifted by at least $13 \mathrm{eV}$ plus the spectral resolution. The targets should also not to have too high redshift such that the O VII triplets will not overlap with the $0.5 \mathrm{keV}$ nitrogen line. Table 5.3 lists the minimum and maximum redshifts for clusters to be observed by the XMS core and the XMS full arrays which meet these requirements.

To calculate the signal of the O VII and O VIII lines for clusters for both the XMSC and XMCF cases, we assume the nominal massive cluster model to be at a redshift of 0.05 . The redshift is chosen such that it is slightly higher than $z_{\min }$ for the XMS full array in Table 5.3. It is also important that there actually be clusters which are at or within the selected redshift which are fairly regular in shape in their outer regions and with masses which are comparable to the cluster model we calculated (e.g., Abell 85, Abell 1795).

To calculate the total count rates of the lines from a cluster at the assumed redshift as observed by $I X O\left(R_{\text {line }}\right)$, we first convolve the rest frame surface brightness of the lines (Figure 5.9) with a top hat function with a width of 0.114 (0.286) Mpc which corresponds to the physical distance at $z=0.05$ covered by the XMSC (XMSF) field of view, and then multiply the convolved rest frame surface brightness by a factor of $A_{\mathrm{eff}} \Omega_{\mathrm{FOV}}(4 \pi)^{-1}(1+z)^{-3}$ to give the count rate $R_{\text {line. }}$. In calculating the signal-tonoise ratios, count rates at a radius where the surface brightness of the weaker line is maximum in the outer regions are used as the optimum model count rates. The 
optimum count rates are also listed in Table 5.2.

We calculate the signal-to-noise ratios for the optimum models (1 NXB, 1 GXB) which use the count rates listed in Table 5.2. The signal for each line is given by $S_{\mathrm{ctn}}=R_{\text {line }} t$, where $t$ is the exposure time. The noise for each line is given by

$$
N=\sqrt{\left(R_{\text {line }}+R_{\text {cont }}+R_{\mathrm{GXB}}+R_{\mathrm{NXB}}\right) t} .
$$

The signal-to-noise ratio of the line ratio is given by

$$
S N(\mathrm{O} \text { VIII/O VII })=\left[(S N(\mathrm{O} \text { VIII }))^{-2}+(S N(\mathrm{O} \text { VII }))^{-2}\right]^{-1 / 2}
$$

where $S N(\mathrm{O}$ VII $)$ and $S N(\mathrm{O}$ VIII) are the signal-to-noise ratios of the O VII and O VIII lines, respectively.

To address the effects of the NXB uncertainties, we also calculate the signal-tonoise ratios for models with the NXB multiplied by a factor of 2 (2 NXB, 1 GXB) and a factor of 0.5 ( $0.5 \mathrm{NXB}, 1 \mathrm{GXB})$. The GXB varies with sky position. To address the uncertainties in the GXB, we vary the GXB by a factor of 0.8 (1 NXB, 0.8 GXB), 1.2 (1 NXB, 1.2 GXB), and 1.5 (1 NXB, 1.5 GXB). In real observations, the line signals may not be optimum. We consider models with the count rates of the lines to be a factor of $0.75\left(0.75 R_{\text {line }}\right), 0.50\left(0.50 R_{\text {line }}\right)$, and $0.25\left(0.25 R_{\text {line }}\right)$ of the optimum models.

The signal-to-noise ratios as a function of exposure time for the different models are shown in Figure 5.11. To ensure that there are enough counts for the lines, we only plot the signal-to-noise ratios if the total count for each line $S_{\mathrm{ctn}}$ is larger than 30.

For the XMSC instrument setup with the optimum model (1 NXB, 1 GXB), in 
order to get a signal-to-noise ratio of 3 for the O VII triplets, about $220 \mathrm{ksec}$ is needed. With a deeper observation of $600 \mathrm{ksec}$, the signal-to-noise ratio can exceed 5 . For the O VIII doublets, a shorter exposure time of $\sim 180 \mathrm{ksec}$ is need to have $S_{\text {ctn }}>30$ with a signal-to-noise ratio of 3.2. About $450 \mathrm{ksec}$ is needed to get a signal-to-noise ratio of 5 , and it is possible to get a signal-to-noise ratio up to 7 with a very deep observation of $900 \mathrm{ksec}$. If no single cluster is observed for such a long exposure, the very deep exposure can be achieved by stacking many observations of outer regions of many clusters. In this case, the line emission measured is average over many clusters. For a given exposure time, increasing the NXB by a factor of 2 or increasing the GXB by a factor of 1.5 only decrease the signal-to-noise ratios by about $10 \%$ for both the O VII or O VIII lines. As expected, decreasing the line signals significantly decreases the signal-to-noise ratios. If the line signals are smaller than half of the optimum values, it will be impossible to detect the O VII (O VIII) line with an exposure time less than $\sim 0.8(0.5)$ Msec. Since the differences in the line ratios between the NEI and CIE models are bigger than an order of magnitude, only a 2 or 3 sigma measurement of the line ratio is sufficient to provide a strong test of the non-equilibrium ionization. Limited by the O VII detection, an exposure time of about $220 \mathrm{ksec}$ is sufficient to have a 2.3- $\sigma$ determination for the line ratio for our optimum model.

For the XMSF instrument setup, with the optimum model (1 NXB, 1 GXB), about $\sim 130$ (100) ksec is needed to get a signal-to-noise ratio of 3 for the O VII triplets (O VIII doublets). A longer exposure of $\sim 350$ (270) ksec is needed to get a signal-to-noise ratio of 5 for the O VII (O VIII) lines, and a very deep exposure of $\sim 1$ (1) Msec is needed for a 8 (10)- $\sigma$ detection. Increasing the NXB by a factor of 2 or increasing the GXB by a factor of 1.5 decreases the signal-to-noise ratios by less than $\sim 13 \%$ for both the O VII and O VIII lines. Similarly to the XMSC instrument 
setup, the signal-to-noise ratios decrease as the line signals decrease. For the optimal model, a shorter exposure time of about $130 \mathrm{ksec}$ (also limited by the O VII detection) compared to the XMSC setup will give the same 2.3- $\sigma$ detection for the line ratio.

Overall, the XMSF instrument setup can achieve higher signal-to-noise ratios for a given exposure time compared to the XMSC setup for the optimum models. This is mainly due to the larger field of view of the detector which can collect more photons with the same exposure time. The XMSF is only slightly subjected to the continuum background uncertainties (cluster continuum emission, NXB, and GXB) due to the poorer spectral resolution. For both the XMSC and XMSF setups, detecting the $\mathrm{O}$ VII and O VIII lines and testing non-equilibrium ionization in cluster outer regions are promising.

\subsection{Discussions and Conclusions}

Studying the physics in the outer regions of clusters is very important to understand how clusters are formed, how the intracluster gas is heated, as well as to constrain the formation of large-scale structure. Because of the very low density in cluster outer regions, the collisional timescales are very long and comparable to the cluster age. Electrons and ions passed through the accretion shocks may not have enough time to reach equipartition and the ions may be under-ionized. In Chapter 3, we have studied the non-equipartition effects on clusters using one-dimensional hydrodynamic simulations. In this chapter, we systematically studied non-equilibrium ionization effects on clusters and the X-rays signatures using the same set of simulations we have developed (Wong \& Sarazin 2009).

By using semi-analytic arguments together with numerical simulations, we have shown that the non-equilibrium ionization effect is nearly independent of cluster mass 
but depends strongly on redshift. In particular, non-equilibrium ionization effects are stronger for low-redshift clusters. Therefore, the brighter massive clusters at lowredshifts are good candidates for studying the non-equilibrium ionization effects.

We systematically studied non-equilibrium ionization signatures in X-rays for a massive cluster with $M_{\mathrm{sh}}=1.53 \times 10^{15} M_{\odot}$. We first calculated the ionization fractions for 11 elements heavier than He following the electron temperature and density evolutions of each fluid element. We then calculated the X-ray emissivity of each fluid element and the resulting projected spectra for the cluster. Since the electron temperature profiles depend on electron heating efficiency $\beta$, we have considered three different possibilities which represent a very low heating efficiency $(\beta=1 / 1800)$, an intermediate heating efficiency $(\beta=0.5)$, and equipartition, $\beta=1$. We also considered models which assume equilibrium ionization for comparison.

At a radius (e.g., $2 \mathrm{Mpc}$ ) where the ionization timescale is long, the overall spectra for the NEI and CIE-Non-Eq models are very similar. This is because of the dominant free-free emission, and both models assume the same electron temperature. However, in the outer regions, e.g, at $r \sim 3.5 \mathrm{Mpc}$ which is between $R_{\text {vir }}$ and $R_{\mathrm{sh}}$, the soft emission in the NEI model is dominated by line emission, where the CIE-Non-Eq spectrum is still dominated by the continuum free-free emission.

By analyzing the surface brightness profiles, we found that soft emission (0.3$1.0 \mathrm{keV}$ ) for the NEI model can be enhanced by more than $20 \%$ at around $3 \mathrm{Mpc}$, and up to nearly an order of magnitude near the shock radius compared to the CIENon-Eq model. The soft emission enhancement is mainly due to the line emission from under-ionized ions. The non-equilibrium ionization effects on the medium (1.0$2.0 \mathrm{keV})$ and hard $(2.0-10.0 \mathrm{keV})$ band emissions are smaller. The overall X-ray band $(0.3-10.0 \mathrm{keV})$ emission is dominated by the soft emission, and the total $\mathrm{X}$ - 
ray emission for the NEI model decrease much slower than that of the CIE-Non-Eq model. Thus, if cluster outer regions are in non-equilibrium ionization, the shock region will be much more luminous compared to the CIE-Non-Eq model.

By inspecting a number of spectra, we found that the most prominent nonequilibrium ionization signature in line emission is the line ratio of the He-like O VII triplets and the H-like O VIII doublets, $S(\mathrm{O}$ VIII $) / S(\mathrm{O}$ VII). The line ratios for the CIE models are higher than 10 for most regions between $r=1-4 \mathrm{Mpc}$, while the line ratios are smaller than 10 for the NEI models. The differences in the line ratios between the NEI and CIE models increase with radius, and the differences are more than an order of magnitude for radii beyond $\sim 2 \mathrm{Mpc}$. These results are insensitive to the degree of non-equipartition or electron heating efficiency $\beta$. We suggest that the line ratios can be used to distinguish between the NEI and CIE models. The electron temperature profile can be determined from fits to the continuum spectra of the outer regions of clusters, allowing the CIE line ratios to be determined. Comparison to the observed ratios should show the effects of non-equilibrium ionization. Note that line ratios of $S(\mathrm{O}$ VIII $) / S(\mathrm{O}$ VII $)<3$ in the outer regions of massive clusters is a clear signal of NEI.

We have also studied the detectability of the O VII and O VIII lines around cluster accretion shock regions with $I X O$, as well as the test for non-equilibrium ionization using the line ratio. For our optimum model, we found that with the XMS core array, an exposure time of $220 \mathrm{ksec}$ is need to have a 3.0- $\sigma$ detection of the O VII lines and about $180 \mathrm{ksec}$ is need to have $>30$ counts for a 3.2- $\sigma$ detection of the O VIII lines. The uncertainties in NXB and GXB will not affect the results significantly. For the XMS full array while we assume the spectral resolution to be the same as the outer array throughout the detector, we found that the signal-to-noise ratios for 
our optimum model are higher for the same exposure time as the XMS core array. In particular, only about 130 (100) ksec is needed to detect the O VII (O VIII) line. The XMS full array is only slightly more subject to the NXB and GXB uncertainties due to the poorer spectral resolution. To test the non-equilibrium ionization model, once the lines have been detected, only a 2 to $3-\sigma$ measurement for the line ratio is needed due to the order of magnitude difference between the NEI and CIE line ratios. We found that an observation with about 130 (220) ksec with the XMS full (core) array is enough to provide a strong test for non-equilibrium ionization with 2.3- $\sigma$ measurement of the line ratio. In summary, detecting the O VII and O VIII lines around the cluster accretion shock regions and testing non-equilibrium ionization in cluster outer regions with $I X O$ are promising.

It is expected that the O VII and O VIII lines from WHIM will also be strong. Because of the high spectral resolution of the XMS, emissions from different redshifts should be easily separated. Only the emission from WHIM immediately surrounding the target cluster will be potentially confused with the emission from cluster outer regions. To observe the $\mathrm{O}$ VII and $\mathrm{O}$ VIII lines and study NEI effects in cluster accretion shock regions, it will be best to avoid observing directions along the filaments where it is believed that denser preheated WHIMs and subclusters are preferentially accreted onto more massive and relaxed galaxy clusters.

What do we learn about clusters from the ionization state of the outer gas? Since collisional ionization and recombination rates involve straightforward atomic physics, the processes are not in question and the rates are reasonably well-known. Unlike shock electron heating or rates for transport processes like thermal conduction, the basic physics is not uncertain and magnetic fields do not affect the results in a significant way. What we mainly learn about is the pre-shock physical state of materials 
which are being accreted by the cluster. If most of the WHIM is ionized beyond O VII, then the effects described in this chapter will be greatly reduced. If most of the material currently being accreted by clusters comes in through filaments which have a higher ionization, then NEI effects will be diminished significantly. If most of the gas being added to clusters at present comes in through mergers with groups which deposit most of the gas in the inner regions of clusters, the gas will achieve CIE quickly.

From the theoretical point of view, with the increasing number of observations of galaxy cluster outer regions $\left(\sim R_{200}\right)$ and the potential to extend observations out to the shock radius with $I X O$ in the future, it is necessary to perform more detailed simulations than ours. It is also interesting to extend our work to study the connections between the shocked ICM and the more diffuse WHIM surrounding clusters. Three-dimensional simulations will be essential to understand the effects of mergers or filament accretion on the degree of ionizations in different regions of clusters. This will allow us to characterize the variation of non-equilibrium signatures in the clusters; such calculations are essential to compare observational signatures with our understanding of the cluster physics near the accretion shocks. Cosmological simulations have been performed recently to study the NEI signatures (Yoshida et al. 2005; Cen \& Fang 2006; Yoshikawa \& Sasaki 2006). These studies have shown that both non-equipartition and non-equilibrium ionization effects are important in cluster outer regions; although they focus more on the lower density and lower temperature WHIM. High resolution simulations were also performed for studying NEI effects in clusters, but these are limited to binary mergers with idealized initial conditions and focus on the denser merger shocks (Akahori \& Yoshikawa 2008, 2010). Re-simulating representative clusters and the surrounding WHIM from cosmological simulations 
with higher resolutions and including realistic physics (e.g., cooling, conduction, turbulent pressure, magnetic pressure, and relativistic support by cosmic rays) will be necessary to provide realistic model images and spectra. The different observational signatures and connections between the ICM and the more diffuse WHIM can also be addressed self-consistently by these simulations. 
Table 5.1. Parameters for the IXO XMS Core and Full Arrays

\begin{tabular}{cccc}
\hline \hline Instrument & $A_{\text {eff }}\left(\mathrm{cm}^{2}\right)$ & $\Omega_{\mathrm{FOV}}\left(\operatorname{arcmin}^{2}\right)$ & $\Delta E(\mathrm{eV})$ \\
\hline $\mathrm{XMSC}$ & 10000 & $2 \times 2$ & 2.5 \\
$\mathrm{XMSF}$ & 10000 & $5 \times 5$ & 10 \\
\hline
\end{tabular}

Note. - Column 3 lists the field of view. Column 4 lists the spectral resolution (FWHM).

Table 5.2. Count Rates

\begin{tabular}{cccccc}
\hline \hline Instrument & Ion & $R_{\text {line }}$ & $R_{\text {cont }}$ & $R_{\mathrm{NXB}}$ & $R_{\mathrm{GXB}}$ \\
\hline XMSC & O VII & 2.11 & 0.210 & 2.44 & 6.09 \\
XMSF & O VII & 11.3 & 4.03 & 46.7 & 117 \\
XMSC & O VIII & 1.66 & 0.171 & 0.878 & 2.19 \\
XMSF & O VIII & 8.69 & 1.43 & 20.8 & 51.8 \\
\hline
\end{tabular}

Note. - Count rates in Columns 3-6 are in unit of $10^{-4}$ counts s $^{-1}$.

Table 5.3. Minimum and Maximum Redshifts

\begin{tabular}{ccccl}
\hline \hline Instrument & $\Delta E_{\min }(\mathrm{eV})$ & $z_{\min }$ & $\Delta E_{\max }(\mathrm{eV})$ & $z_{\max }$ \\
\hline $\mathrm{XMSC}$ & 15.5 & 0.0278 & 58.1 & 0.116 \\
$\mathrm{XMSF}$ & 23.0 & 0.0417 & 50.6 & 0.0992 \\
\hline
\end{tabular}

Note. - Column 2 lists the minimum energy shifts in order for the O VII triplets to be separated from Galactic lines. Column 3 lists the corresponding redshifts of $\Delta E_{\min }$. Column 4 lists the maximum energy shifts in order for the O VII triplets not to overlap with the $0.5 \mathrm{keV}$ nitrogen line. Column 5 lists the corresponding redshifts of $\Delta E_{\max }$. 


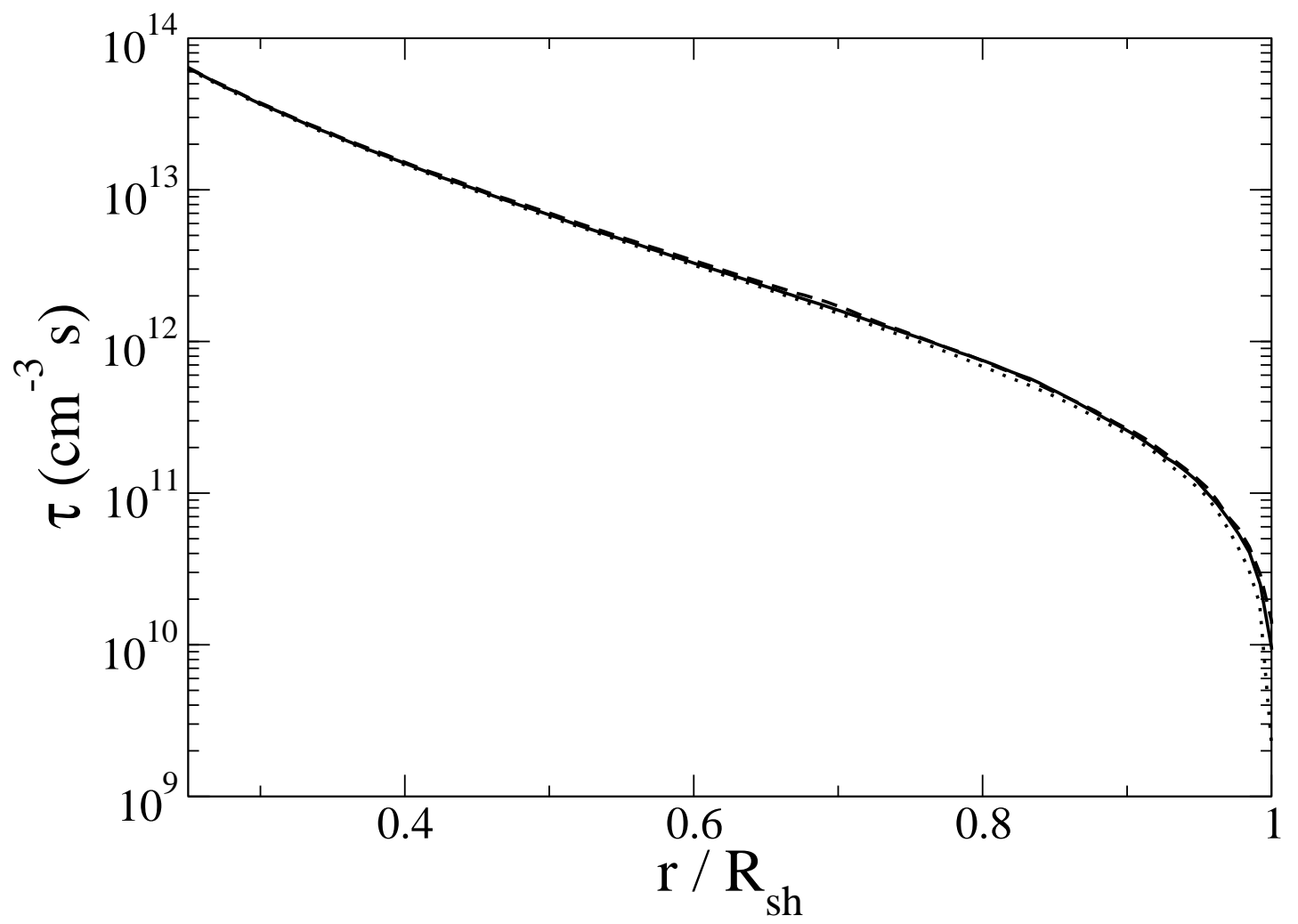

Fig. 5.1. - Ionization timescale parameter $\tau$ (Equation (5.2)) versus radius $r$ scaled to the cluster shock radius $R_{\mathrm{sh}}$ for cluster models with total accreted masses of $M_{\mathrm{sh}}=$ 0.77 (dashed line), 1.53 (solid line), and 3.06 (dotted line) $\times 10^{15} M_{\odot}$ at a redshift of $z=0$. The shock radii for the three clusters from small to high mass are $R_{\mathrm{sh}}=$ 3.31, 4.22, and 5.41 Mpc, respectively. All three lines lie almost on top of one another. 


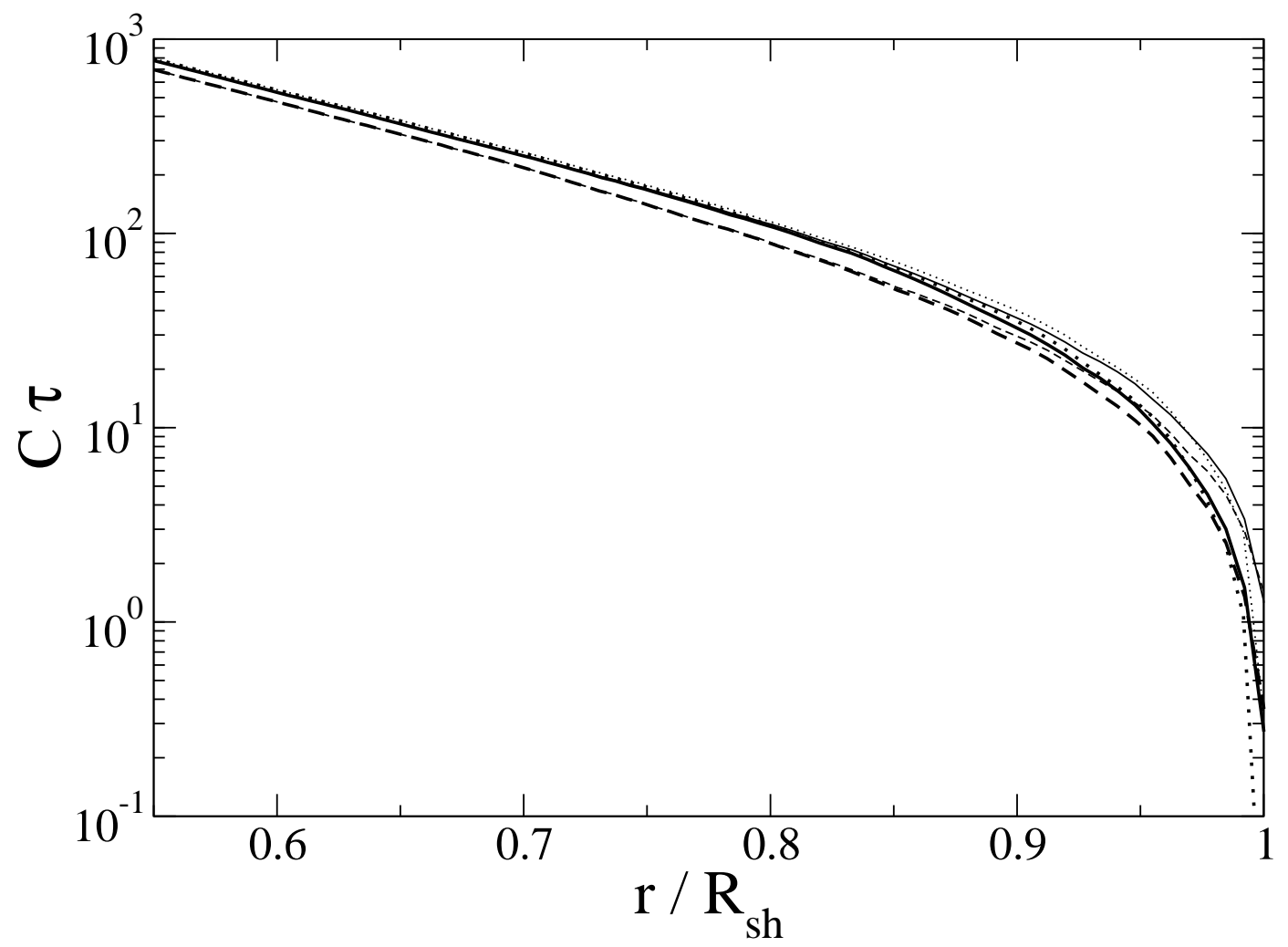

Fig. 5.2.- Dimensionless ionization parameter $C_{i}\left(T_{e}\right) \tau$ for O VII versus the scaled radius $\left(r / R_{\mathrm{sh}}\right)$ for clusters with total accreted masses of $M_{\mathrm{sh}}=0.77$ (dashed line), 1.53 (solid line), and 3.06 (dotted line) $\times 10^{15} M_{\odot}$ at a redshift of $z=0$. Models with $\beta=1 / 1800$ (non-equipartition) and $\beta=1$ (equipartition) are shown in thick and thin lines, respectively. 


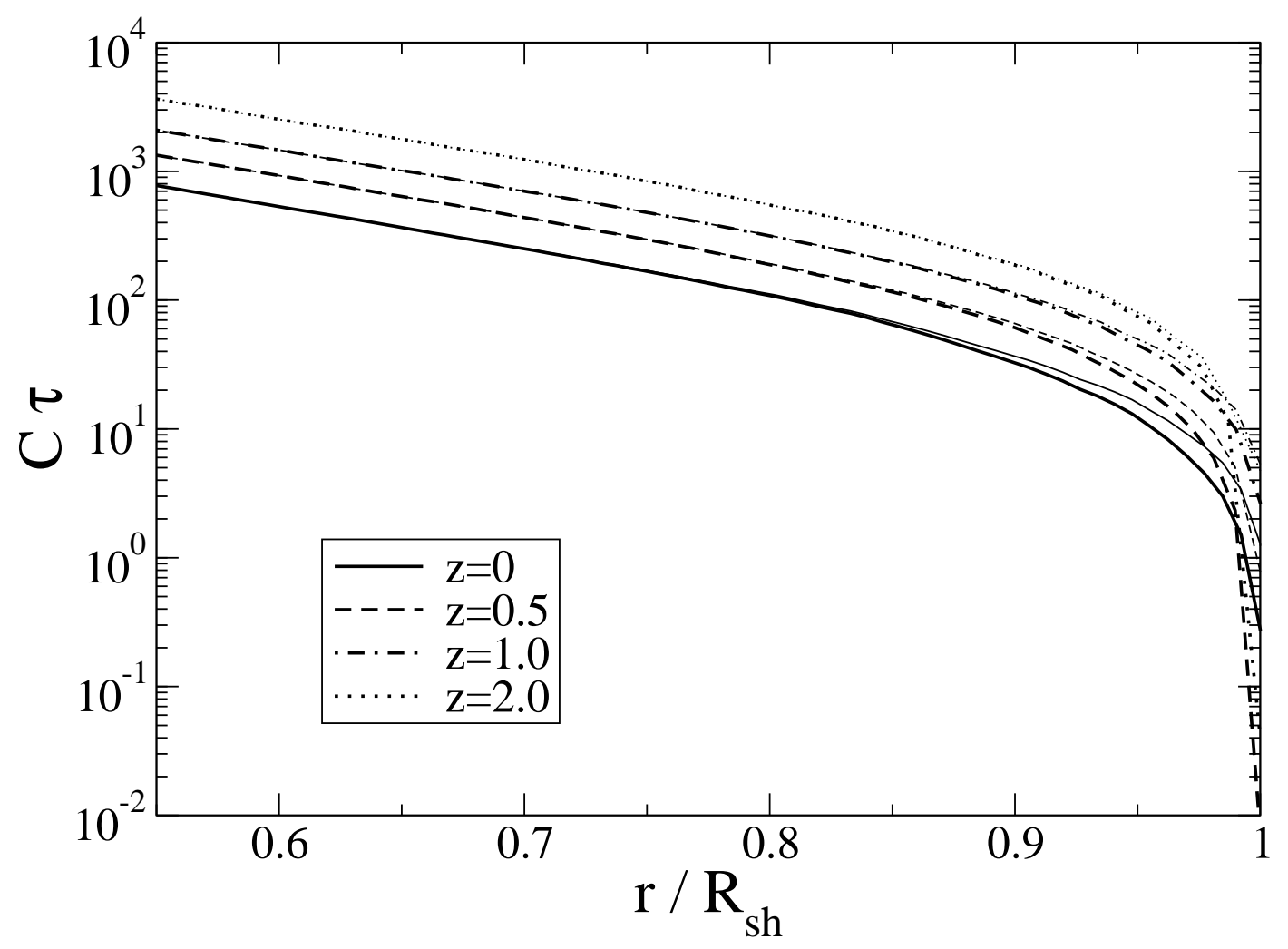

Fig. 5.3.- Dimensionless ionization parameter $C_{i}\left(T_{e}\right) \tau$ for O VII versus the scaled radius $\left(r / R_{\mathrm{sh}}\right)$ for clusters at different redshifts. The cluster model with a total accreted mass $M_{\mathrm{sh}}=1.53 \times 10^{15} M_{\odot}$ at $z=0$ is used. Models with $\beta=1 / 1800$ (non-equipartition) and $\beta=1$ (equipartition) are shown in thick and thin lines, respectively. 


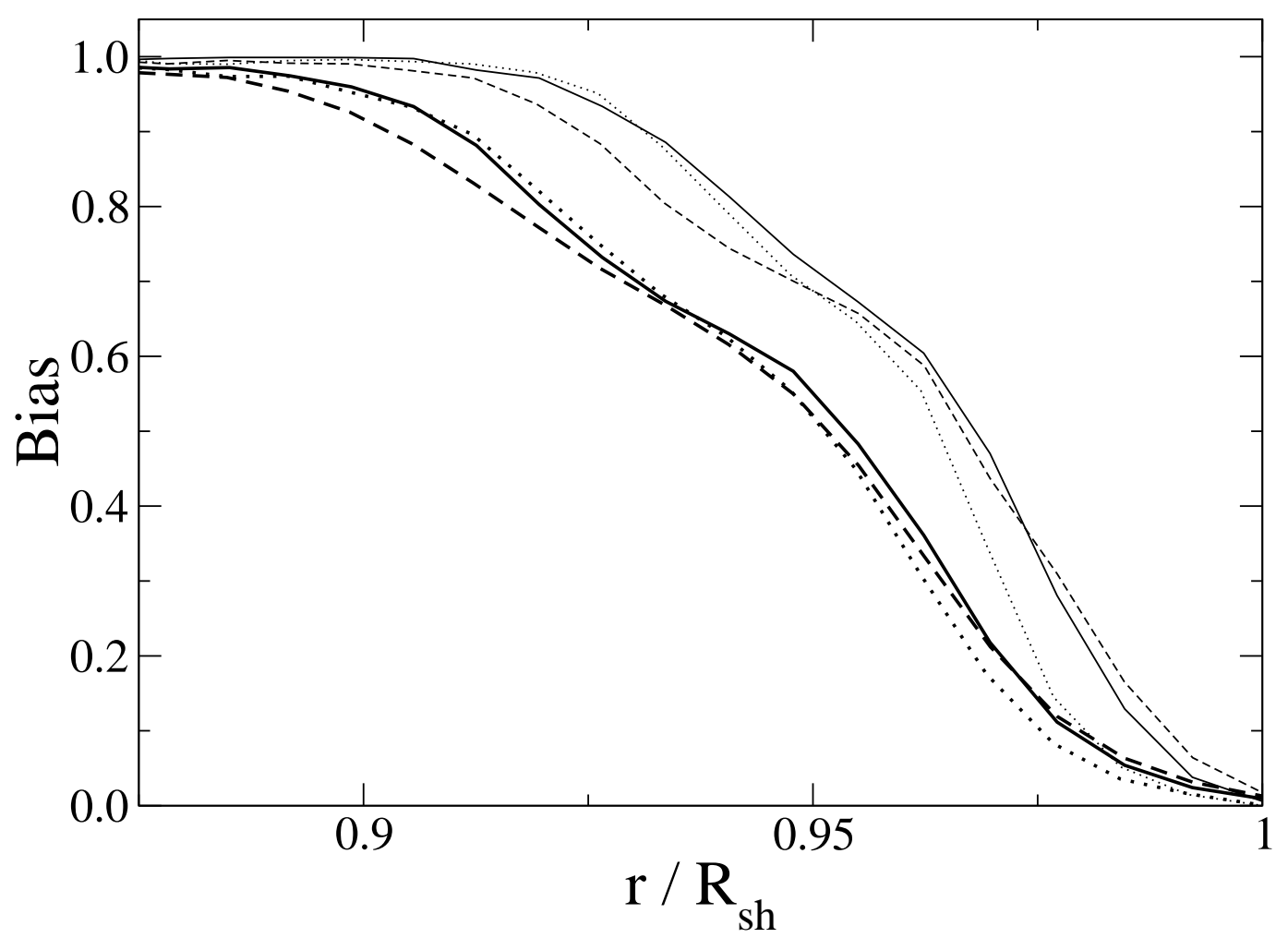

Fig. 5.4. - Non-equilibrium ionization bias versus the scaled radius $\left(r / R_{\mathrm{sh}}\right)$ for clusters with total accreted masses of $M_{\mathrm{sh}}=0.77$ (dashed line), 1.53 (solid line), and 3.06 (dotted line) $\times 10^{15} M_{\odot}$ at a redshift of $z=0$. Models with $\beta=1 / 1800$ (non-equipartition) and $\beta=1$ (equipartition) are shown in thick and thin lines, respectively. 


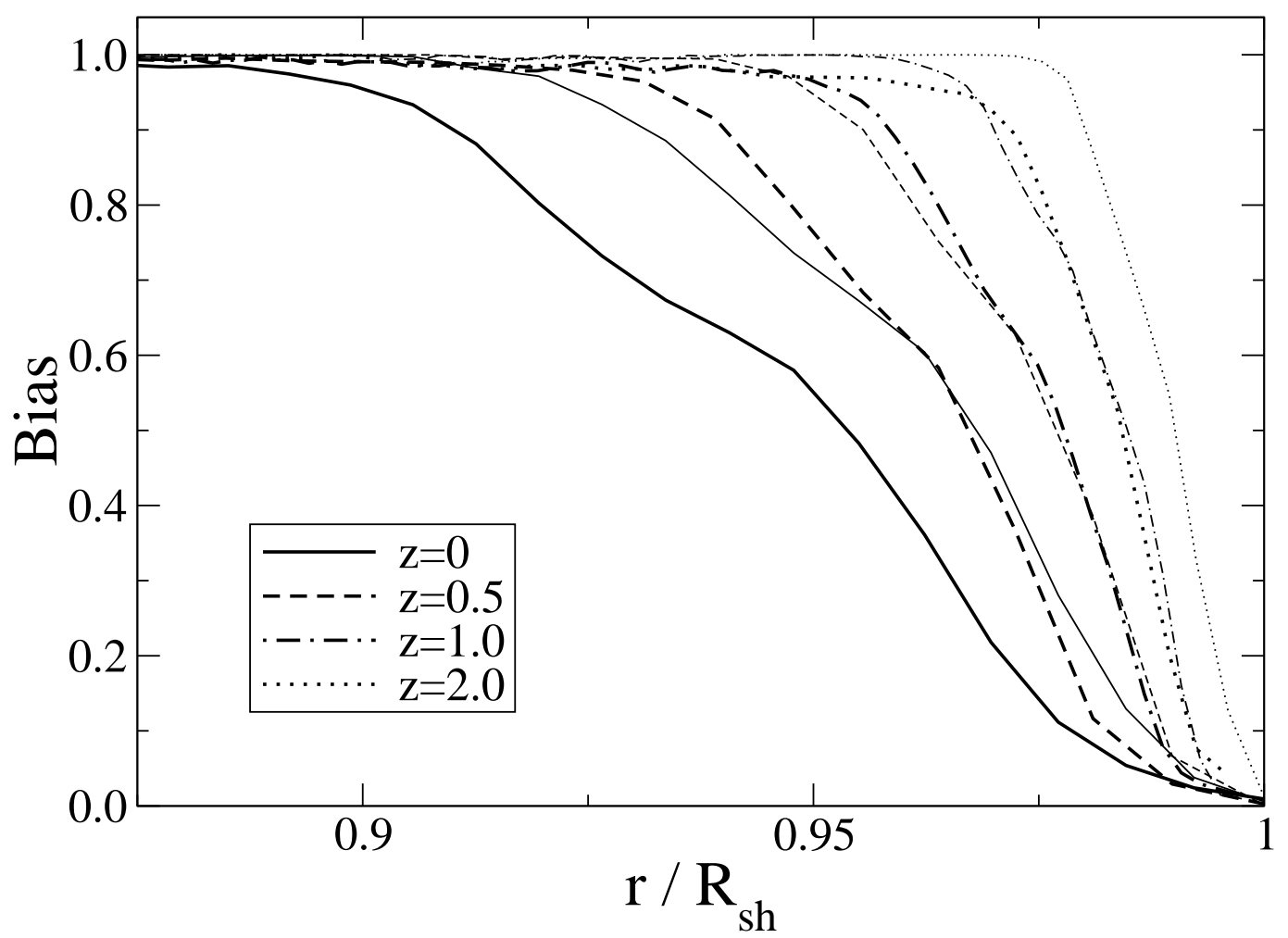

Fig. 5.5.- Non-equilibrium ionization bias versus the scaled radius $\left(r / R_{\mathrm{sh}}\right)$ for clusters at different redshifts. The cluster model with a total accreted mass $M_{\mathrm{sh}}=$ $1.53 \times 10^{15} M_{\odot}$ at $z=0$ is used. Models with $\beta=1 / 1800$ (non-equipartition) and $\beta=1$ (equipartition) are shown in thick and thin lines, respectively. 


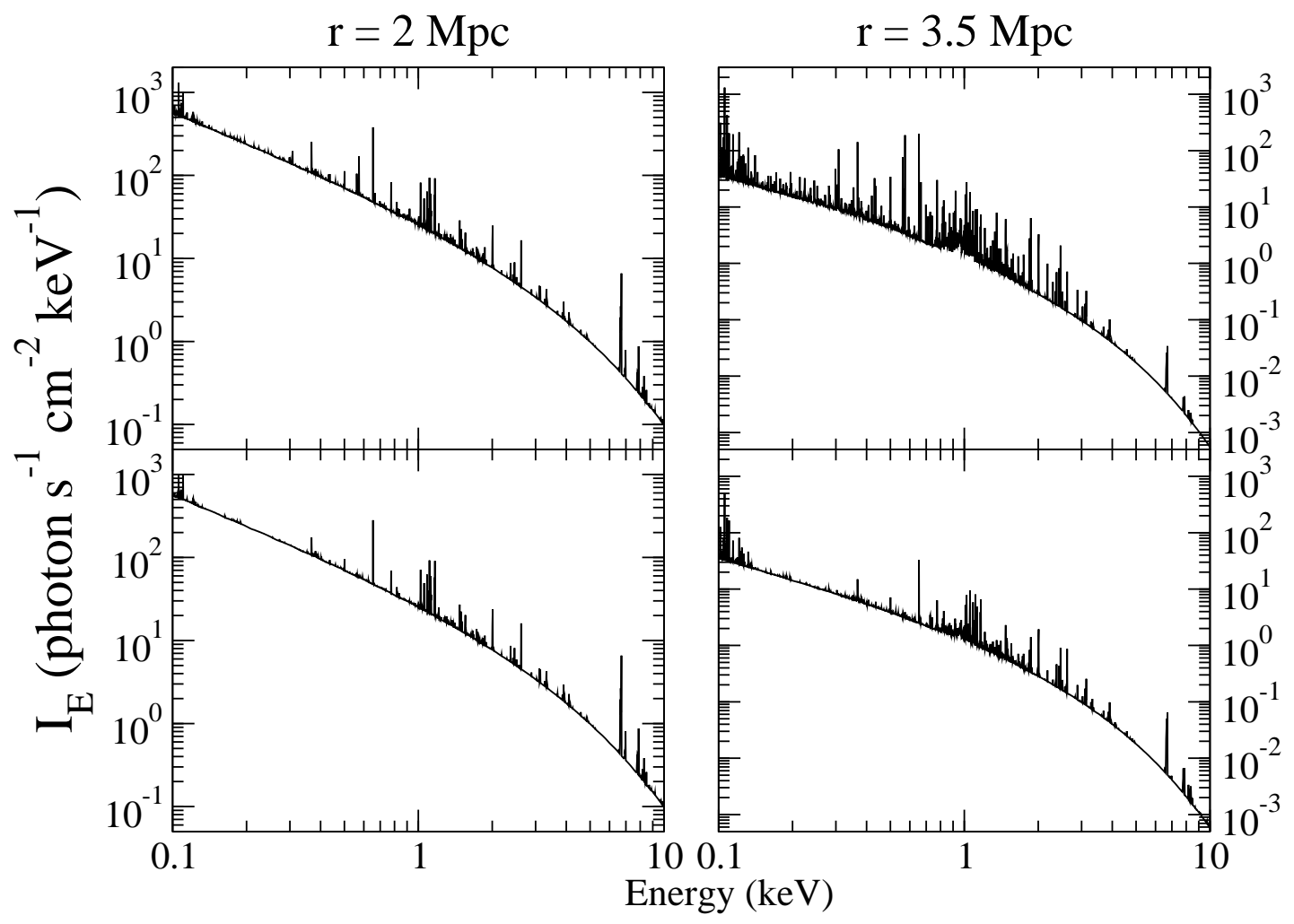

Fig. 5.6. - Projected rest frame spectra for the cluster model with an accreted mass of $M_{\mathrm{sh}}=1.53 \times 10^{15} M_{\odot}$ at $z=0$ are shown at two projected radii (left: $2 \mathrm{Mpc}$; right $3.5 \mathrm{Mpc}$ ). Upper panels: the NEI model with $\beta=1 / 1800$ and non-equipartition of electrons and ions. Lower panels: projected rest frame spectra for the CIE model with the same $\beta$ and non-equipartition. 


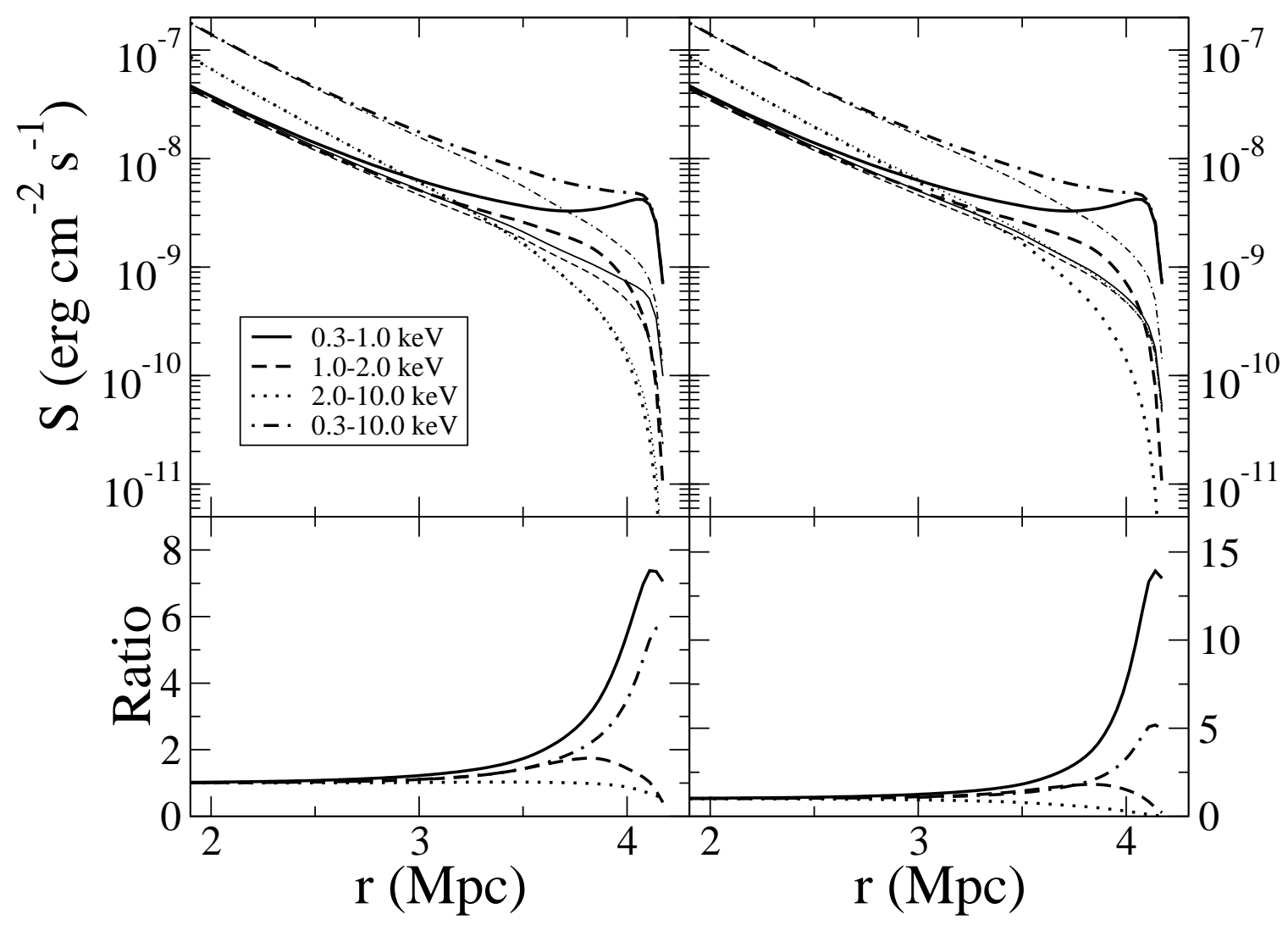

Fig. 5.7.- Upper left panel: rest frame projected surface brightness profiles for different energy bands for the NEI model with $\beta=1 / 1800$ (thick lines) and the CIENon-Eq model (thin lines). Upper right panel: rest frame projected surface brightness profiles for the NEI model with $\beta=1 / 1800$ (thick lines) and the CIE-Eq model (thin lines). Lower left panel: ratios of the surface brightness profiles $S_{\mathrm{NEI}} / S_{\mathrm{CIE}-\mathrm{Non}-\mathrm{Eq}}$. Lower right panel: ratios of the surface brightness profiles $S_{\mathrm{NEI}} / S_{\mathrm{CIE}-\mathrm{Eq}}$. 


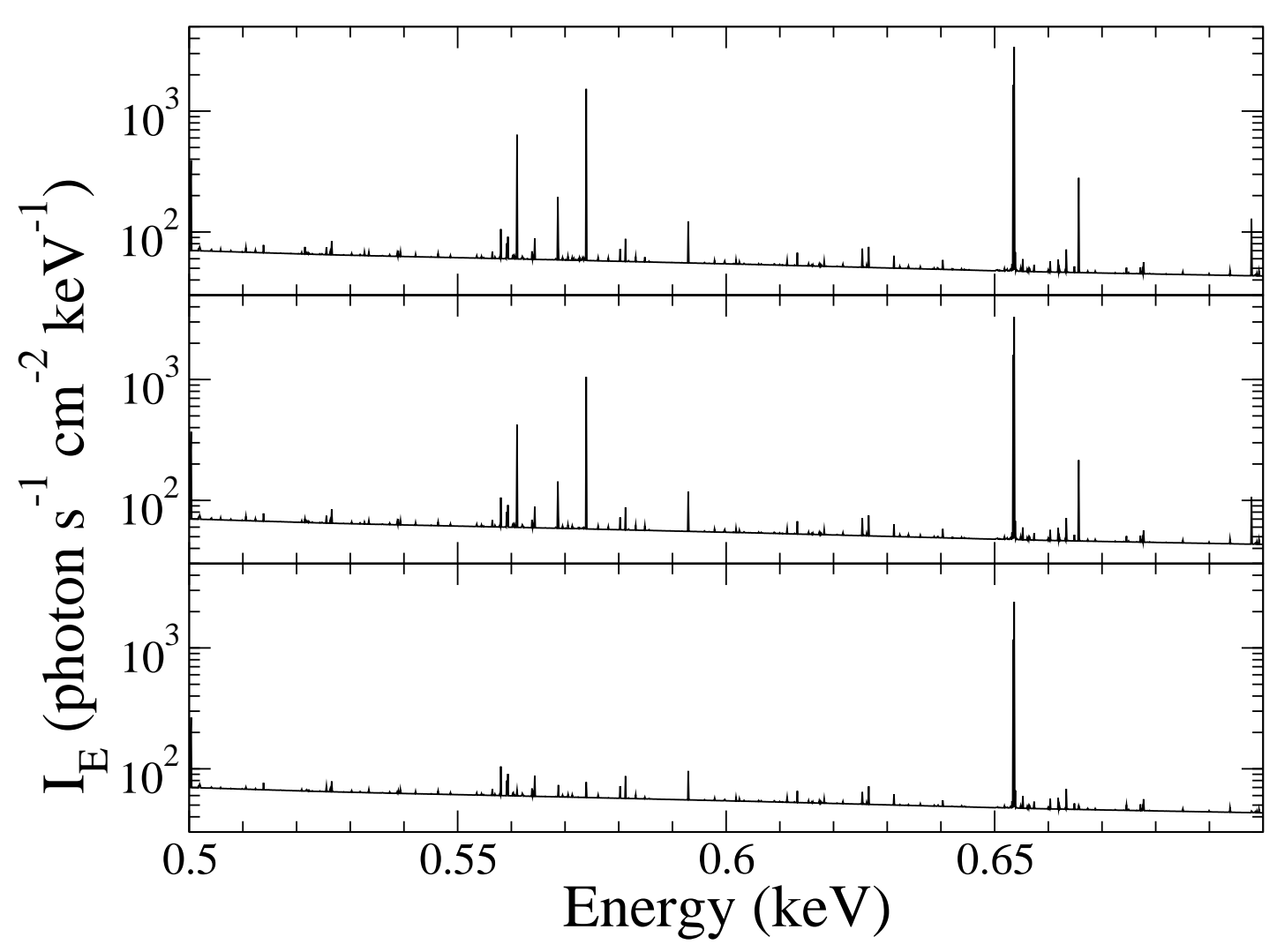

Fig. 5.8. - Upper panel: projected rest frame spectra for the NEI model with $\beta=$ 1/1800. Middle panel: projected rest frame spectra for the NEI model with $\beta=$ 0.5. Lower panel: projected rest frame spectra for the CIE-Non-Eq model with $\beta=1 / 1800$. All spectra are for the $M_{\mathrm{sh}}=1.53 \times 10^{15} M_{\odot}$ at $z=0$, and are for a projected radius of $r=2 \mathrm{Mpc}$. The spectra are binned with $\Delta E=0.1 \mathrm{eV}$. 


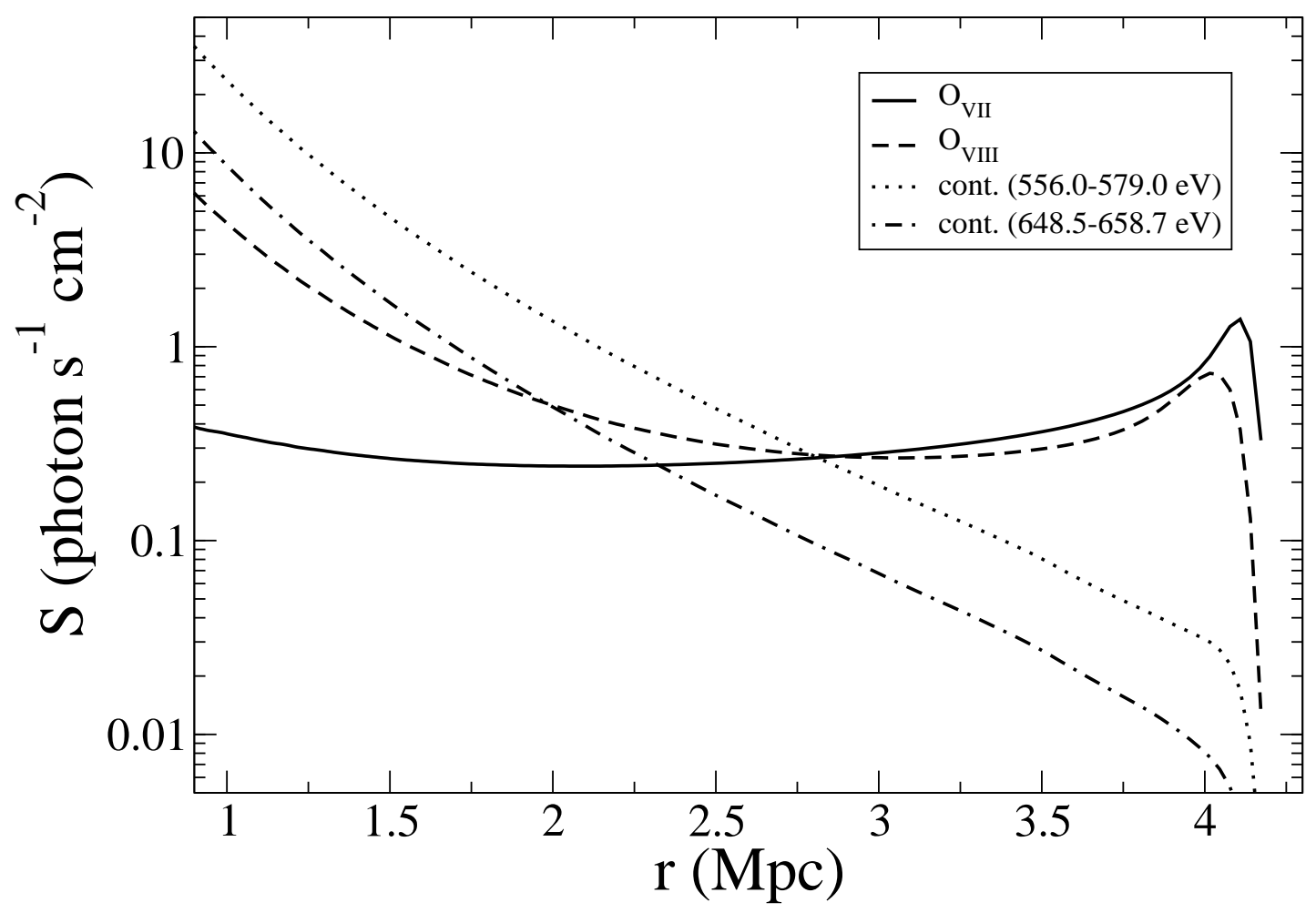

Fig. 5.9. - Surface brightness of the O VII and O VIII lines for the NEI model with $\beta=1 / 1800$ as a function of projected radius. The continuum emission within the narrow energy bands covering the lines is also shown. 


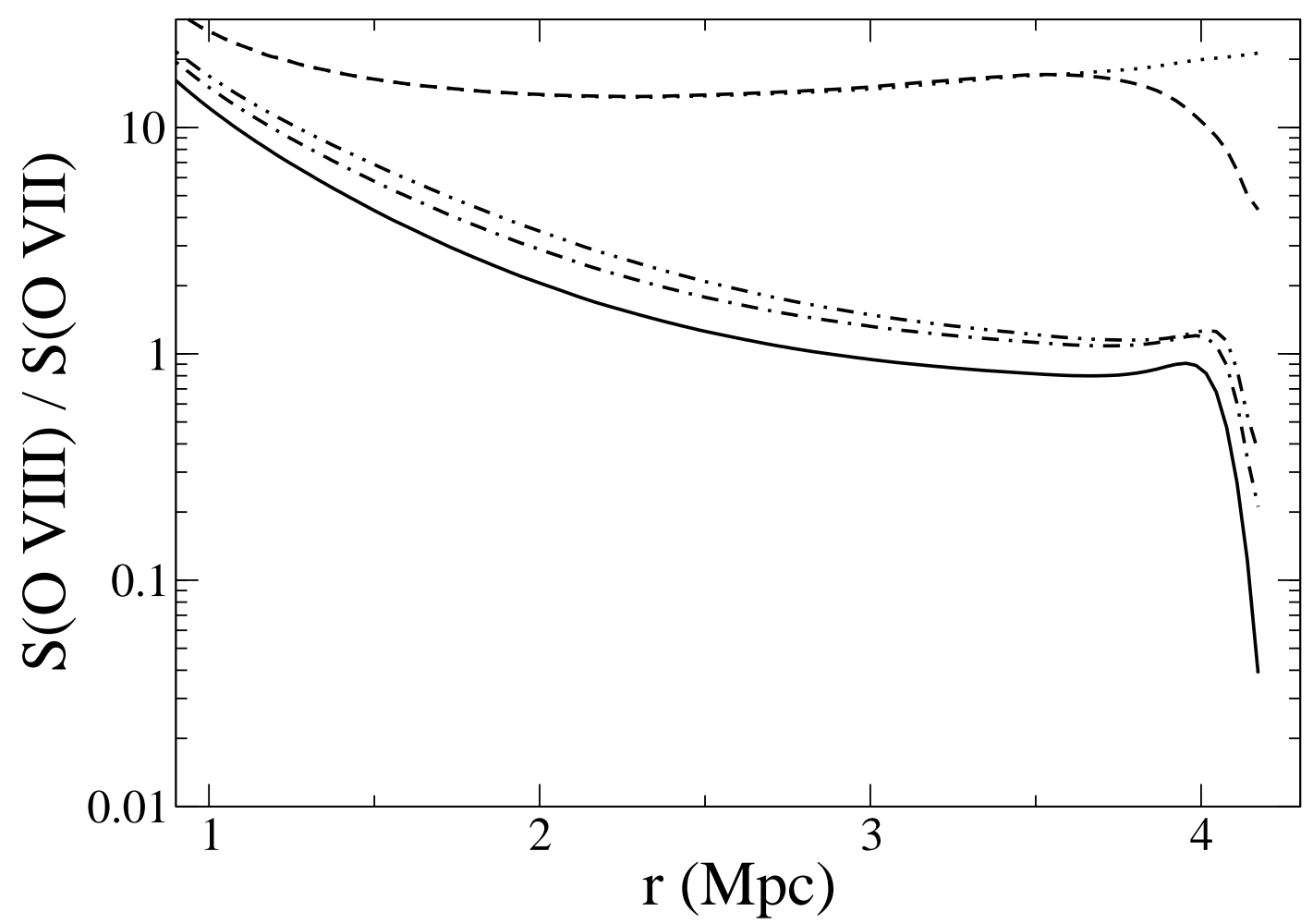

Fig. 5.10. - Line ratios $S(\mathrm{O}$ VIII $) / S(\mathrm{O}$ VII) for the NEI models with $\beta=1 / 1800$ (solid), 0.5 (dash-dotted), and 1.0 (dash-dot-dotted). Line ratios for the CIE-Non-Eq (dashed) and CIE-Eq (dotted) models are also shown. 


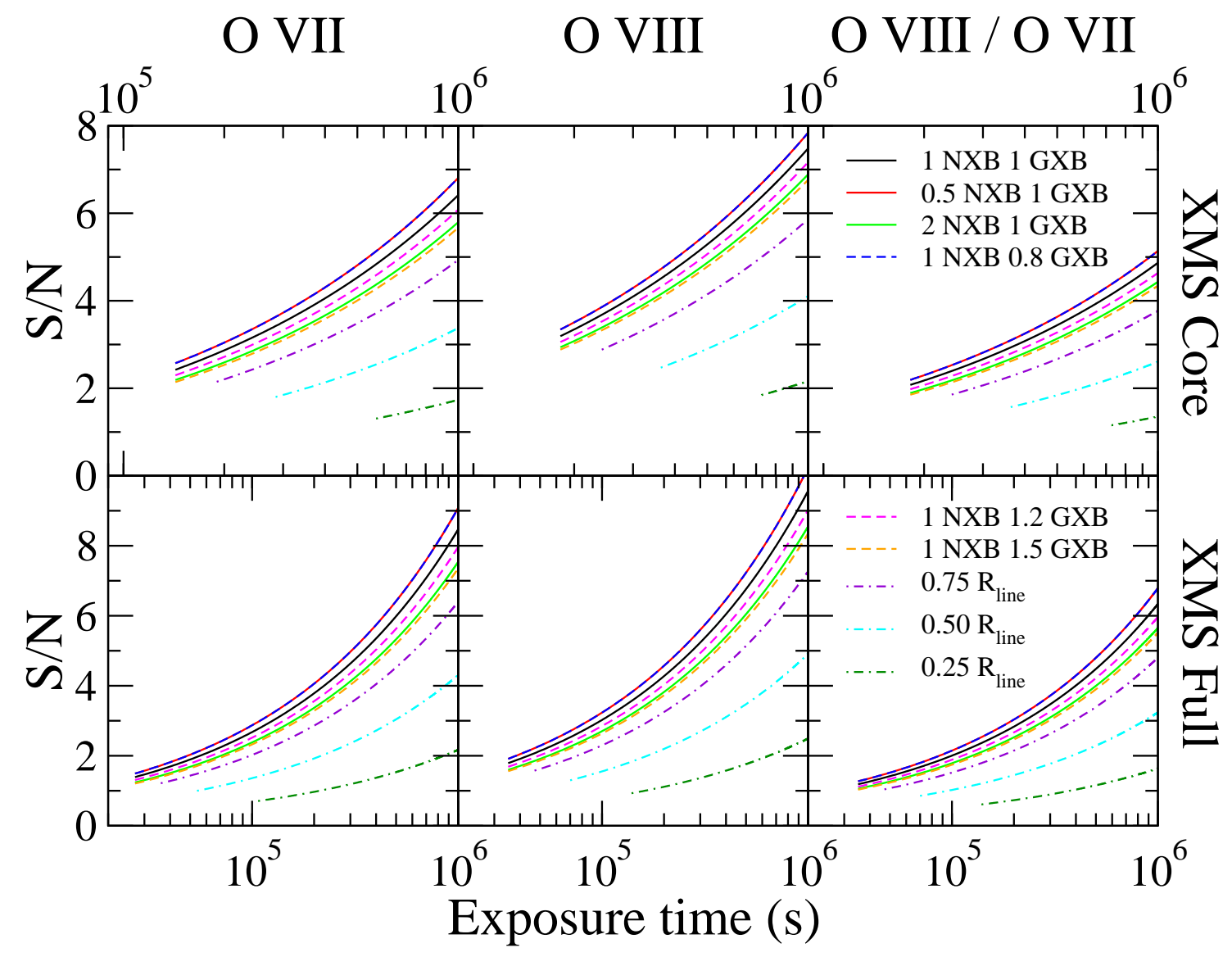

Fig. 5.11. - Left (middle) panels show the signal-to-noise ratios for the O VII triplets (O VIII doublets) expected to be detected by $I X O$. The right panels show the signalto-noise ratios for the O VII and O VIII line ratios. The upper panels correspond to the XMSC, and the lower panels correspond to the XMSF. Different signal and noise levels are assumed for the different models. The legends for the lines are the same for all panels, but are separated into two panels due to the space limitation. 


\section{Chapter 6}

\section{Conclusions and Future Directions for Research}

In this thesis, the effects of non-equilibrium processes in clusters of galaxies have been assessed. With improvements in the sensitivities of X-ray and SZ to measurements, signatures of non-equilibrium processes in clusters of galaxies should be observable in the near future. A careful study of these non-equilibrium physical effects is hence useful. Non-equilibrium processes such as non-equipartition of electrons and ions may have an impact on precision cosmological studies. If one wants to understand cluster physics at a percentage level and study cosmology using clusters to a comparable precision, studying non-equilibrium effects on precision cosmology is important.

Motivated by the importance of clusters of galaxies as cosmological probes, we have studied non-equilibrium processes in clusters of galaxies on two different size scales. On small size scales $(r \lesssim 50 \mathrm{kpc}$ to $r \sim 500 \mathrm{kpc})$, we have studied observationally the cooling core cluster Abell 2626 with AGN feedback. On large size scales ( $r \gtrsim 2 \mathrm{Mpc}$ ), we have studied theoretically non-equilibrium processes (in particular, non-equipartition and non-equilibrium ionization) in the outermost accretion 
shock regions of galaxy clusters. We summarize our work and present some possible directions for future research below.

\subsection{Summary}

\section{Abell 2626: Interacting Radio Jets and Cooling Core with Jet Precession}

In Chapter 2, we have presented a detailed analysis of the XMM-Newton and Chandra observations of Abell 2626 focused on the X-ray and radio interactions. Within the region of the radio mini-halo $(\sim 70 \mathrm{kpc})$, there are substructures which are probably produced by the central radio source and the cooling core. Within this area, the cD IC 5338 and the S0 IC 5337 are the two most remarkable galaxies. From the Hubble Space Telescope (HST) archive, we found that the $\mathrm{cD}$ galaxy has two distinct nuclei separated by less than $\sim 5 \mathrm{kpc}$ in projection. On radio images, the most unusual features are the two elongated radio bars to the north and south of the unresolved central radio core of the $\mathrm{cD}$ galaxy. These two unusual amorphous, symmetric radio "bars" run parallel at opposite sides of the $\mathrm{cD}$ galaxy. We find that there is no obvious correlation between the radio bars and the X-ray image. The morphology of Abell 2626 is more complex than that of the standard X-ray radio bubbles seen in other cool core clusters. Thus, Abell 2626 provides a challenge to models for the cooling flow-radio source interaction. We identified two soft X-ray (0.3-2 keV) peaks with the two central cD nuclei. One of them has an associated hard X-ray $(2-10 \mathrm{keV})$ point source, and it is likely that this is the active radio AGN. We suggest that the two symmetric radio bars can be explained by two precessing jets ejected from the AGN. Beyond the cluster center, we find two regions of extended Xray emission to the southwest and northeast of the cluster center which are apparently associated with merging subclusters. The main Abell 2626 cluster and these two 
subclusters are extended along the direction of the Perseus-Pegasus supercluster, and we suggest that Abell 2626 is preferentially accreting subclusters and groups from this large-scale structure filament. We also find an extended X-ray source associated with the cluster S0 galaxy IC 5337; the morphology of this source suggests that it is infalling from the west, and is not associated with the southwest subcluster, as had been previously suggested.

\section{Non-Equipartition Effects in the Outer Regions of Relaxed Clusters}

The collisionless accretion shock at the outer boundary of a galaxy cluster may primarily heat the ions instead of electrons since the ions carry most of the kinetic energy of the infalling gas. Near the accretion shock, the density of the intracluster medium is very low and the electron-ion Coulomb collisional timescale is longer than the accretion timescale. Electrons and ions may not achieve equipartition in these regions.

Chapter 3 presents the studies on the effects of electron-ion non-equipartition in the outer regions of relaxed clusters for a wide range of masses in the $\Lambda$ CDM cosmology using one-dimensional hydrodynamic simulations. The effects of the nonadiabatic electron heating efficiency, $\beta$, on the degree of non-equipartition are also studied. Using the gas fraction $f_{\text {gas }}=0.17$ (which is the upper limit for a cluster), we give a conservative lower limit of the non-equipartition effect on clusters. We have shown that for a cluster with a mass of $M_{\text {vir }} \sim 1.2 \times 10^{15} M_{\odot}$, electron and ion temperatures differ by less than a percent within the virial radius $R_{\text {vir }}$. The difference is $\approx 20 \%$ at $\sim 1.4 R_{\text {vir }}$ for a non-adiabatic electron heating efficiency in the range of $\beta \sim 1 / 1800$ to 0.5 . Beyond that radius, the non-equipartition effect depends strongly

on $\beta$, and this strong dependence at the shock radius can be used to distinguish shock heating models or constrain the shock heating efficiency of electrons. 
With our simulations, we have also studied systematically the signatures of nonequipartition on X-ray and SZ observables. We have calculated the effect of nonequipartition on the projected temperature and X-ray surface brightness profiles using the MEKAL emission model. We found that the effect on the projected temperature profiles is larger than that on the deprojected (or physical) temperature profiles. The non-equipartition effect can introduce a $\sim 10 \%$ bias in the projected temperature at $R_{\mathrm{vir}}$ for a wide range of $\beta$. We also found that the effect of non-equipartition on the projected temperature profiles is enhanced by increasing the metallicity. In the low-energy band $\lesssim 1 \mathrm{keV}$, the non-equipartition model surface brightness can be higher than that of the equipartition model in the cluster outer regions. Future X-ray observations extending to $\sim R_{\text {vir }}$ or even close to the shock radius should be able to detect these non-equipartition signatures.

For a given cluster, the difference between the SZ temperature decrements for the equipartition and the non-equipartition models, $\delta \Delta T_{\mathrm{SZE}}$, is larger at a higher redshift. For the most massive clusters at $z \approx 2$, the differences can be $\delta \Delta T_{\mathrm{SZE}} \approx 4-5 \mu \mathrm{K}$ near the shock radius. We also found that for our model in the $\Lambda$ CDM universe, the integrated SZ bias, $Y_{\text {non-eq }} / Y_{\text {eq }}$, evolves slightly (at a percentage level) with redshift, which is in contrast to the self-similar model in the Einstein-de Sitter universe. We discussed briefly whether the equipartition and non-equipartition models near the shock region can be distinguished by future radio observations with, for example, the Atacama Large Millimeter Array (ALMA).

\section{Impact of Non-Equipartition Effects on Precision Cosmology}

Using the same set of hydrodynamical simulations presented in Chapter 3, we have calculated three potential systematic biases in the $Y$ versus mass relations introduced by non-equipartition effects during the cross-calibration or self-calibration when us- 
ing the galaxy cluster abundance technique to constraint cosmological parameters (Chapter 4). We used a semi-analytical technique to estimate the non-equipartition effects on the distribution functions of $Y$ ( $Y$ functions) determined from the extended Press-Schechter theory. Depending on the calibration method, we found that nonequipartition effects can induce systematic biases on the $Y$ functions, and the values of the cosmological parameters $\Omega_{M}, \sigma_{8}$, and the dark energy equation of state parameter $w$ can be biased by a few percent. In particular, non-equipartition effects can introduce an apparent evolution in $w$ of a few percent for all of the techniques for calibrating the samples which we considered. Methods are suggested to take into account the non-equipartition effect empirically when using the cluster abundance technique to study precision cosmology. We conclude that systematic uncertainties in the $Y$ versus mass relation of even a few percent can introduce a comparable level of biases in cosmological parameter measurements.

\section{Non-Equilibrium Ionization Effects in the Outer Regions of Relaxed Clus- ters}

The densities in the outer regions of clusters of galaxies are very low, and the collisional ionization timescales are very long. As a result, heavy elements will be under-ionized after they have passed through the accretion shock. In Chapter 5, we studied the effects of non-equilibrium ionization for relaxed clusters in the $\Lambda \mathrm{CDM}$ cosmology using one-dimensional hydrodynamic simulations. We found that nonequilibrium ionization effects do not depend significantly on the cluster mass but do depend strongly on redshift. We gave an analytic, self-similar argument which reproduces this behavior. The effects are stronger for clusters at lower redshifts. We present X-ray signatures such as surface brightness profiles and emission lines in detail for a massive cluster at low redshift. In general, soft emission $(0.3-1.0 \mathrm{keV})$ 
is enhanced significantly by under-ionization, and the enhancement can be nearly an order of magnitude near the shock radius. The most prominent non-equilibrium ionization signature we found is the O VII and O VIII line ratio. The ratio for non-equilibrium ionization and collisional ionization equilibrium models are different by more than an order of magnitude at radii beyond half of the shock radius. These non-equilibrium ionization signatures are equally strong for models with different nonadiabatic shock electron heating efficiencies. We have also calculated the detectability of the O VII and O VIII lines with the future International X-ray Observatory $(I X O)$, and we conclude that an exposure of $\sim 130-220 \mathrm{ksec}$ on a moderate-redshift, massive regular cluster with the X-ray Microcalorimeter Spectrometer (XMS) on the IXO will be sufficient to provide a strong test for the non-equilibrium ionization model. These measurements can be used to determine the pre-shock ionization state of the material (presumably WHIM) accreted by clusters.

\subsection{Future Directions for Research}

\section{Abell 2626 and AGN Feedback Problem}

The most interesting structure of Abell 2626 is the two parallel radio bars on the opposite sides of the cD galaxy IC 5338. We suggested that this radio structure may be due to jet precession. It would be very useful to do numerical simulations of precessing jets in cluster cool cores to see if they can produce similar structures. In fact, after our work was published, there have been a number of high resolution hydrodynamic simulations carried out to study the jet precession scenario (Sternberg \& Soker 2008; Falceta-Gonçalves et al. 2010). These simulations have confirmed that precessing jets can inflate bubbles in ICM which are often seen in cooling core clusters, although the simulations were not aiming at reproducing the observations 
of Abell 2626. In one of these simulations (Figure 1(c) in Falceta-Gonçalves et al. 2010), the simulated projected energy map of the cooling core shows very similar structures to the two radio bars of Abell 2626, although to compare the synchrotron emission correctly, a full MHD simulation will be necessary. These simulations may also address observational signatures such as line broadening by the turbulence that may be induced by the precessing jets. This line broadening may potentially be observed by future X-ray observatories such as Astro- $H^{1}$ or the IXO.

On the observational side, a deeper observation certainly will help to better understand the dynamical states of Abell 2626. Because of the small spatial scale within the cooling core regions, Chandra will be ideal due to its excellent resolution. A deep enough observation would allow us to create a high resolution $\left(\lesssim 10^{\prime \prime}\right)$ temperature map to search for complicated structures due to the jet-gas interaction. This is not possible with our existing observations. Jet-like features could also be resolved much better, allowing us to better study the interaction between the X-ray ICM and the radio jets. We can also search for hard X-ray emission from the northeast nucleus which was not detected in our observations.

It will also be important to search for and to compare systems which also show signatures in jet precessions. In fact, there is a number of cooling core clusters which shows evidence in jet precession (e.g., Churazov et al. 2000; Gitti et al. 2006). A detailed comparison between the properties will help to test and/or constrain the precession scenarios. For example, we found that the cD galaxy in Abell 2626 has two nuclei. It will be interesting to see if all jet precessing candidates have more than one nucleus in their cD galaxies.

Our results show rather strong evidence that the S0 galaxy IC 5337 is falling into the cD galaxy, and the cold gas of the S0 is stripped out by the ICM. Unfortunately,

\footnotetext{
${ }^{1}$ http://astro-h.isas.jaxa.jp/
} 
our observations do not have sufficient statistics to constrain the stripping properties. A deeper observation would allow us to determine the stripped gas temperature, infall velocity, and abundances in the stripped gas. The latter measurement will allow us to estimate the level of metal enrichment of the ICM by gas stripping from infalling galaxies.

On a larger spatial scale, we found that there is a merger signature between the main cluster and the subcluster in the southwest direction. A deeper observation with $X M M-N e w t o n$ would allow us to confirm our interpretation, constrain the properties of the subclusters. and estimate the merger kinematics.

\section{Understanding the Physics of Non-Equilibrium Processes in Galaxies Clus- ters and the Impacts on Cosmology and Large-Scale Structures}

The outer regions of galaxy clusters are ideal sites to study non-thermal and non-equilibrium physics. These studies not only can increase our understanding of cosmology, but also can provide information on the physics of galaxy clusters and plasma physics under extreme conditions.

For the non-equipartition problem near the accretion shock regions, our results shows that the effect on $T_{e}$ is less than $1 \%$ at $R_{200} \sim 0.7 R_{\mathrm{vir}}$. Recent three-dimensional hydrodynamical simulations drawn for a cosmological background have found that the bias can be $\sim 5 \%$ (model CL104 in Rudd \& Nagai 2009). A detailed comparison is needed to address the difference, but a possible explanation may be that in a threedimensional cluster embedded in large-scale structure, the accretion shock can form further in. If three-dimensional results are confirmed, non-equipartition effects will be more important that the results we presented, and will have a bigger impact on precision cosmology.

We have shown that using the cluster abundance to constraint the dark energy 
equation of state requires a full understanding of the systematic uncertainties in galaxy cluster physics. Even though we are only considering the systematic uncertainties introduced by the non-equipartition effect, our results also suggest that systematic uncertainties in the observable-mass relations (e.g., the integrated Comptonization parameter versus mass relation) introduced by other physics of even a few percent can introduce a comparable level of biases in cosmological parameter measurements. Future cluster surveys aiming to constrain departures from general relativity will need to control systematic uncertainties down to a sub-percentage level (Schmidt et al. 2009), and hence cluster physics must be understood in a comparable accuracy. Future theoretical calculations and numerical simulations should pay particular attention to the effects of non-thermal and non-equilibrium physics on the electron pressure profiles. Potential additional systematic uncertainties include conduction, turbulent pressure, magnetic pressure, and relativistic pressure from cosmic rays.

Deep observations should also be carried out to constrain all these effects in detail for individual clusters. One may make use of the archive data from Suzaku to determine the average temperature profile of the outer regions by stacking the spectra for a number of clusters. To minimized systematic uncertainties due to point source contamination, we should select regions observed with Chandra or XMM-Newton. Even if the Chandra and/or XMM-Newton observations cannot detect the faint diffuse cluster emission, they can be used to exclude or model the point source contamination specific to the observed region. The average temperature can be compared to that of the non-equipartition and equipartition models (which should also be stacked with the corresponding masses).

It is important to study the effect of pre-shock materials on non-equilibrium ionization. Observations of the ionization state in the outer regions of clusters can 
constrain the physical state of the pre-shock materials which are being accreted by the cluster. For example, if most of the WHIM is ionized beyond O VII, then the non-equilibrium ionization effects will be greatly reduced. If most of the material currently being accreted by clusters comes in through filaments which have a higher ionization, then non-equilibrium ionization effects will be diminished significantly. If most of the gas being added to clusters at present comes in through mergers with groups which deposit most of the gas in the inner regions of clusters, the gas will achieve collisional ionization quickly.

It is likely that there are non-thermal (non-Maxwellian) electrons in the cluster accretion shock regions which are accelerated by non-thermal processes. It will be important to test if non-thermal electrons will affect the ionization significantly. A first step to handle the non-thermal electron is to assume that the electron distribution can be approximated by superposition of thermal (Maxwellian) distribution with a range of temperatures, as long as the non-thermal electrons are not dominated by relativistic electrons. Relativistic corrections to ionization cross sections will be needed if this is not the case.

From the theoretical point of view, with the increasing number of observations of galaxy cluster outer regions $\left(\sim R_{200}\right)$ and the potential to extend observations out to the shock radius with, e.g., IXO, in the future, it is necessary to perform more detailed simulations than ours. It is also interesting to extend our work to study the connections between the shocked ICM and the more diffuse WHIM surrounding clusters. Three-dimensional simulations will be essential to understand the effects of mergers or filament accretion on the degree of ionizations in different regions of clusters. This will allow us to characterize the variation of non-equilibrium signatures in the clusters; such calculations are essential to compare observational signatures with 
our understanding of the cluster physics near the accretion shocks. Cosmological simulations have been performed recently to study the non-equipartition or/and nonequilibrium ionization signatures (Yoshida et al. 2005; Cen \& Fang 2006; Yoshikawa \& Sasaki 2006). These studies have shown that both non-equipartition and nonequilibrium ionization effects are important in cluster outer regions, although they focus more on the lower density and lower temperature WHIM. High resolution simulations were also performed for studying NEI effects in clusters, but these are limited to binary mergers with idealized initial conditions and focus on the denser merger shocks (Akahori \& Yoshikawa 2008, 2010). Re-simulating representative clusters and the surrounding WHIM from cosmological simulations with higher resolutions and including realistic physics (e.g., cooling, conduction, turbulent pressure, magnetic pressure, and relativistic support by cosmic rays) will be necessary to provide realistic model images and spectra. The different observational signatures and connections between the ICM and the more diffuse WHIM can also be addressed self-consistently with these simulations.

The non-equilibrium calculational techniques can also be extended to study ionization states of many other astrophysical objects. In fact, they are routinely applied to supernovae remnants; the techniques we used were adapted from those used for supernovae remnants (Hughes \& Helfand 1985; Borkowski et al. 1994). Non-equilibrium ionization may also be important in accretion disks around stellar mass or supermassive compact objects, and in the cooling cores of galaxy clusters which can be over-ionized by diffusion of hot electrons present. If photoionization is important in any of these objects, a different method in solving the ionization fraction will be needed.

Thus, we foresee a rich future for non-equilibrium studies of diffuse astrophysical 
plasmas in clusters of galaxies both observationally and theoretically. 


\section{Appendix A}

\section{Can Non-Equipartition at Cluster Accretion Shocks be Detected with} ALMA?

Assuming that the shock positions can be determined by other means, ALMA may be able to distinguish between equipartition and non-equipartition models near the shock region with signal to noise ratio as high as 7 for a $\sim 70 \mathrm{hr}$ observation (Section 3.6.4). However, in real observations, the shock radius may not be known. We have to fit the shock radius based on observations, and this decreases the signal to noise ratio. In this chapter, we investigate whether non-equipartition effect can be distinguished by ALMA in the visibility domain. The visibility domain or $u v$ plane is the domain in which measurements (visibilities) are made by radio interferometers (Pearson 1999). Compared to the image domain, the error in the visibility domain can be characterized more easily. This is because, unlike in the image domain, the measurement errors in the visibility domain are largely uncorrelated. Another advantage of data analysis in the visibility domain is that model fitting in the visibility domain is not complicated 
by deconvolution process which is required in the image domain.

Since the SZ signal is stronger at higher redshift and for more massive cluster, we study the extreme case with cluster mass $M_{\mathrm{sh}}(z=2)=1.34 \times 10^{15} M_{\odot}$ at $z=2$ to see if non-equipartition effect can be detected with ALMA. From the one dimensional non-equipartition SZ temperature distortion we have calculated in Section 3.6.4, we generated a $30^{\prime} \times 30^{\prime}$ two dimensional SZ image assuming azimuthally symmetry (Figure A.1). Linear interpolation was used to generate the image with $1024 \times 1024$ pixels. We calculate the complex visibility function measured on a baseline with coordinates $(u, v)$. The complex visibility function $V(u, v)$ is related to the Fourier transform of the sky brightness distribution $I(l, m)$ by (Pearson 1999)

$$
V(u, v)=\int_{-\infty}^{\infty} \int_{-\infty}^{\infty} A(l, m) I(l, m) e^{-2 \pi i(u l+v m)} d l d m
$$

where $l$ and $m$ are the sky coordinates in the image domain, $u$ and $v$ are baseline coordinates or spatial frequencies in the visibility domain, and $A(l, m)$ is the primary beam response. We assume a Gaussian beam with FWHM $=\lambda / D$, where $\lambda$ is the operating wavelength assumed to be $35 \mathrm{GHz}$, and $D$ is the diameter for the $12-\mathrm{m}$ dish. The primary beam is centered at the shock radius shown in Figure A.1. The radial SZ profiles with and without primary beam correction along one of the directions are shown in Figure A.2. The Fourier transform was done by Fast Fourier Transform (Figures A.3 and A.4).

The simulated $u v$ data sampled by ALMA are assumed to be taken from the most compact configuration of 50 dishes (A. Wootten 2009, private communications). We assume that the cluster was directly overhead that it had a declination equal to the latitude of ALMA, and was observed at a zero hour angle. The $u v$ coverage for the simulated observation is shown in Figure A.5. We did not include the effect 
caused by rotation of the Earth in our simulations; this is adequate for our purpose. Including the rotation of the Earth would turn the point in Figure A.5 into arcs. This would actually improve the uv coverage, but probably makes little difference for a circular cluster. We also ignore the effect of bandwidth due to the non-monochromatic radiation and hence equation (A.1) has to be modified by averaging over a finite frequency width (Bridle \& Schwab 1999). The net effect of bandwidth is to smear the signals that are away from the delay-tracking center. Each of the sampled $u v$ data is assigned with a thermal noise of $\sigma_{T}=T_{\text {sys }} / \sqrt{2 \Delta \nu t_{\exp }}$ (Wrobel \& Walker 1999), where $\Delta \nu$ is the frequency bandwidth, $t_{\exp }$ is the exposure time, and the dish temperature is taken to be $T_{\text {sys }}=30 \mathrm{~K}$ for each dish ${ }^{1}$. The system efficiency is ignored in our simulations. We assume the frequency bandwidth to be $16 \mathrm{GHz}$ and the exposure time to be 400 hours for our simulations. We assume point sources can be removed using the long baseline response, and hence, point source contaminations are not included in the simulations. The confusion by unresolved point sources and intrinsic CMB anisotropy is of the order of a few $10^{-9}(1000 / l) \mathrm{K}$ (Holder \& Carlstrom 2001), which is much smaller than the thermal noise for typical spatial frequency sampled by ALMA and for the $400 \mathrm{hr}$ observation we assumed. Hence, confusion is not included in our simulations.

We performed Monte Carlo simulations to generate 10,000 simulated data set including errors. For each simulated data, we fit the real and imaginary parts of the visibility functions simultaneously to both an equipartition model and a nonequipartition model in the $u v$ domain. We assume self-similar profiles of the models of the form

$$
\Delta T_{\text {model }}=N \Delta T\left(N_{R} r\right)
$$

\footnotetext{
${ }^{1}$ http://www.almaobservatory.org/en/about-alma/essentials/numbers
} 
where $r$ is the radius, and $N$ and $N_{R}$ are the SZ temperature and the radius normalizations to be fitted, respectively. The function $\Delta T$ on the right hand side is taken from either the equipartition model or non-equipartition model calculated in Section 3.6.4. In the fittings, we limit the range of spatial frequency of $\left(u^{2}+v^{2}\right)^{1 / 2} \leq 5000$ to maximize the signal to noise ratio. We also include the zero-spacing flux at $(u, v)=(0,0)$ sampled by the Atacama Compact Array (ACA) in the fittings because the main ALMA array alone cannot sample the large-scale SZ signal of the galaxy cluster. The thermal noise of the zero-spacing flux is assumed to be smaller than the other visibility measurements by antenna pairs. In particular, we assume the thermal noise of the zero-spacing flux measured by four 12-m dishes of the ACA to be $\sigma_{T, \mathrm{ACA}}=T_{\mathrm{sys}} / \sqrt{4 \Delta \nu t_{\exp }}$. In real observations, the noise of the zero-spacing flux will probably be lower due to the larger number of dishes of the ACA. The fitting procedure is done by minimizing the chi-square

$$
\chi^{2}=\sum\left|\frac{\operatorname{data}(u, v)-\operatorname{model}(u, v)}{\sigma(u, v)}\right|^{2},
$$

where $\sigma$ is the statistical uncertainty assumed to be given by the thermal noise. One of the simulated runs and the best fitted data are shown in Figure A.6. The residuals, which are defined as the best fitted model minus the raw input data (Figure A.3), along the direction of $v=0$ are shown in Figure A.7. The average reduced chisquares of the 10,000 Monte Carlo simulation runs for the equipartition and nonequipartition models are listed in Table A.1. For the non-equipartition model, the average reduced chi-square is near 1, which indicates that on average, the fitting is as good as expected. The average SZ temperature and radius normalizations are also close to 1 , which indicates that there are no biases introduced. For the equipartition model, the average reduced chi-square is 1.309. This is slightly bigger than for the non- 
equipartition model. From the f-test, the probability that the fit to the equipartition model is better than the fit to the non-equipartition model is $1.712 \times 10^{-3}$. In another word, non-equipartition model is distinguishable from the equipartition model at a 3.3- $\sigma$ level. Clearly, in real observations when there are other uncertainties, the signal-to-noise ratio will be even lower. Also, it is unlikely that any real cluster will be exactly spherical, and that this will make the detection even more difficult. We conclude that it will be very difficult to distinguish the non-equipartition model and equipartition model in a single observation of a cluster with ALMA. Even if the two models may not be distinguishable, non-equipartition effects will introduce a bias in the measured shock radius of $\sim 3 \%$. More constraints on the model or cluster properties may help to reduce the observation time and increase the signal to noise ratio. On the other hand, future radio array such as the Square Kilometer Array (SKA) may have enough sensitivity to distinguish the two models.

Table A.1. Visibility Fitting

\begin{tabular}{ccccc}
\hline \hline Model & d.o.f. & $\left\langle N_{R}\right\rangle$ & $\langle N\rangle$ & $\left\langle\bar{\chi}^{2}\right\rangle$ \\
\hline Non-equipartition & 476 & 0.999 & 1.002 & 1.001 \\
Equipartition & 476 & 0.965 & 1.038 & 1.309 \\
\hline
\end{tabular}




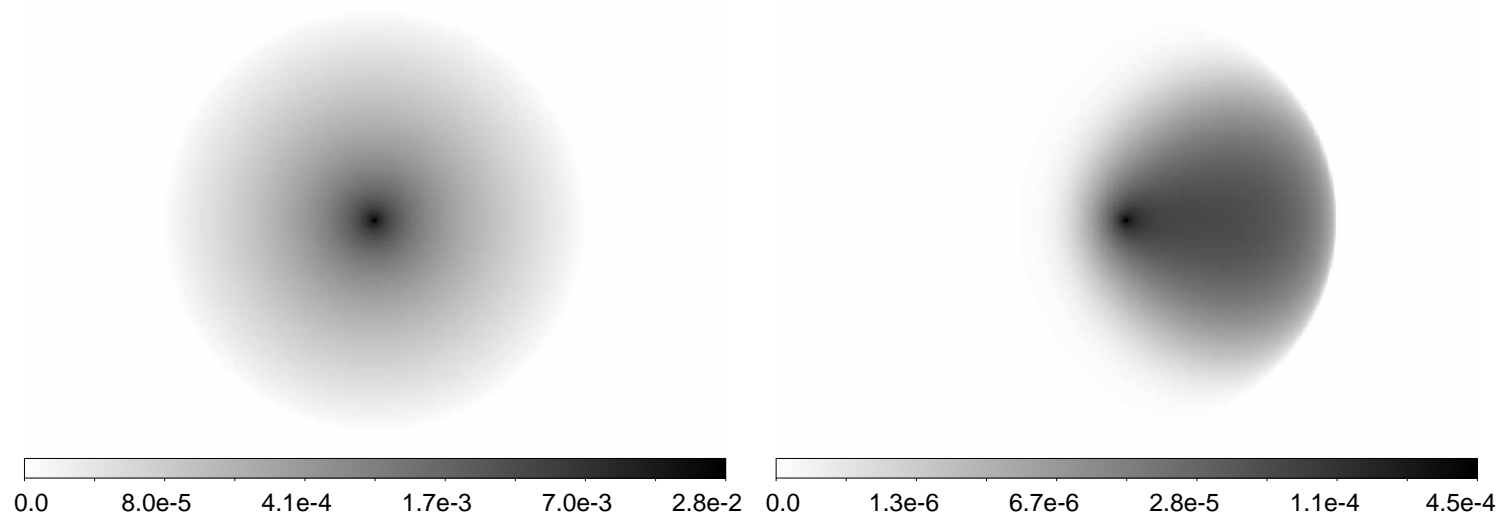

Fig. A.1.- (Left panel) Central region of the SZ image for the non-equipartition model in angular (rather than $u v$ ) coordinates. The edge of the circle indicates the shock radius which is about $180^{\prime \prime}$ from the cluster center. The intensity bar is in units of K. (Right panel) Same as the left panel, but the primary beam response with a Gaussian beam with FWHM $=2.5^{\prime}$ has been applied. The primary beam is centered at the shock radius on the right hand side of the cluster.

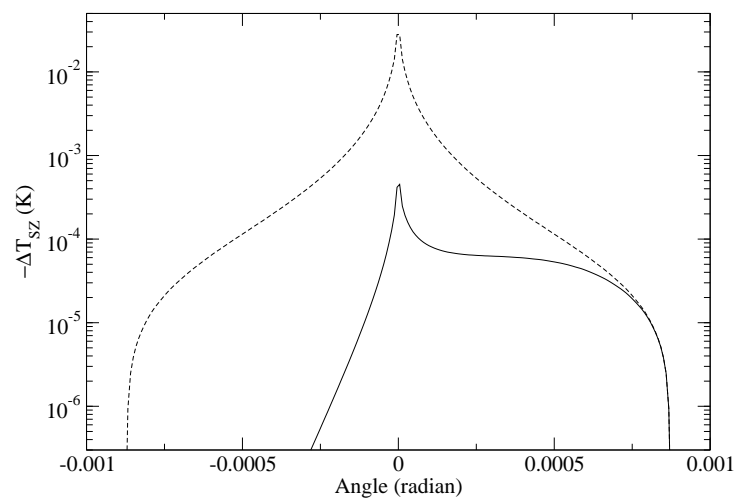

Fig. A.2.- Radial profiles along the horizontal axis of the left panel (dashed line) and the right panel (solid line) in Figure A.1. 

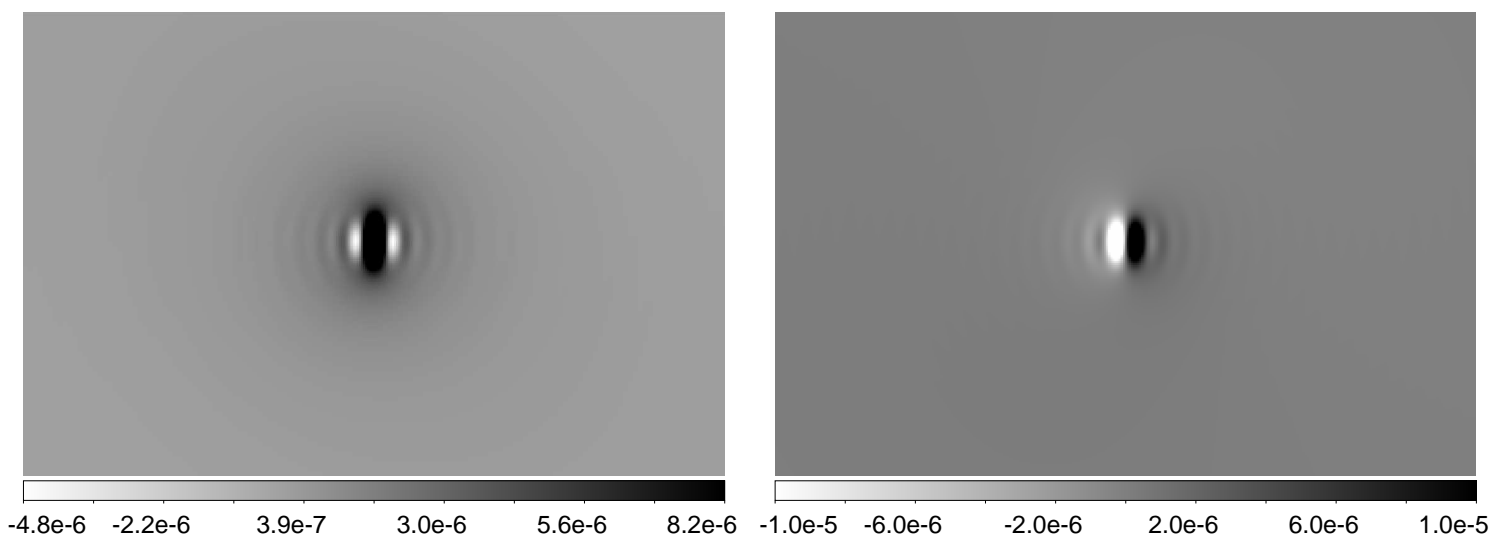

Fig. A.3.- (Left panel) Real part of the visibility function for the image in the right panel in Figure A.1. (Right panel) Imaginary part of the visibility function. The visibility function has been normalized by the primary beam area, so that the intensity bars in both panels are in unit $\mathrm{K}$.

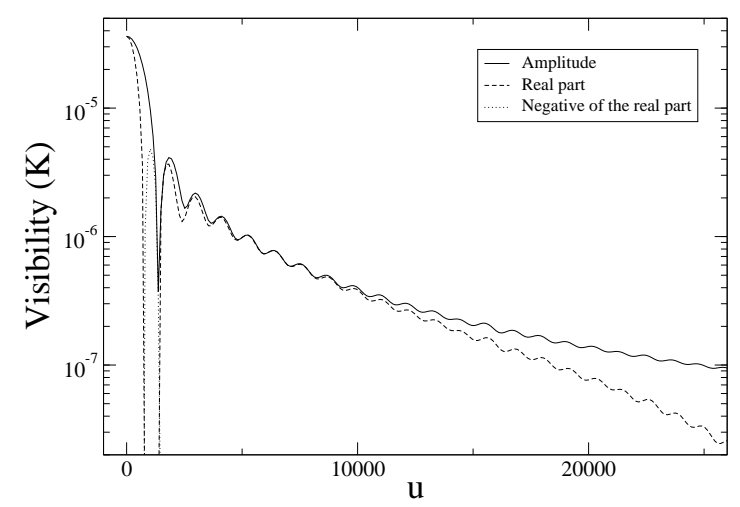

Fig. A.4.-Visibility functions for positive $u$ at $v=0$. The solid line is the amplitude, the dashed line is the real part, and the dotted line is the negative of the real part. The imaginary part is not plotted on the figure. 


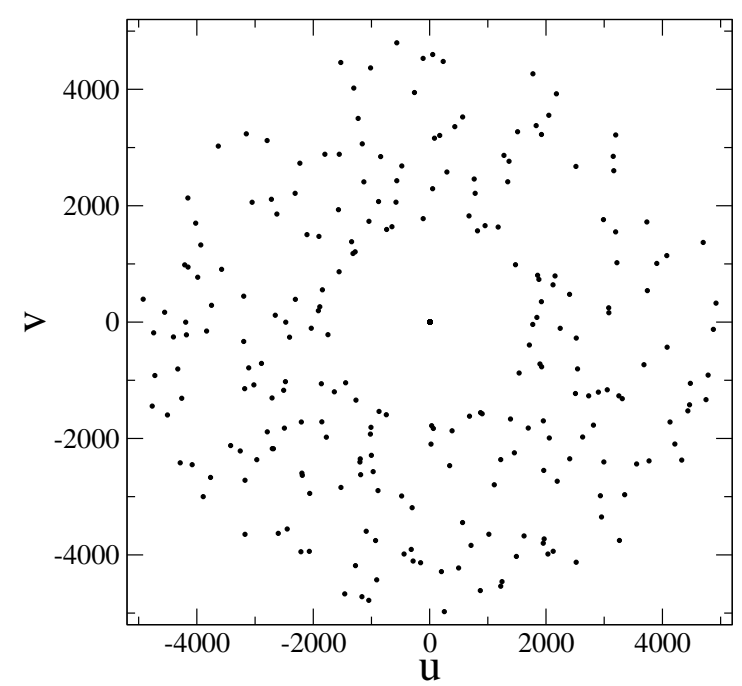

Fig. A.5.- $u v$ coverage of the most compact configuration of ALMA with $\sqrt{u^{2}+v^{2}}$ $\leq 5000$. 

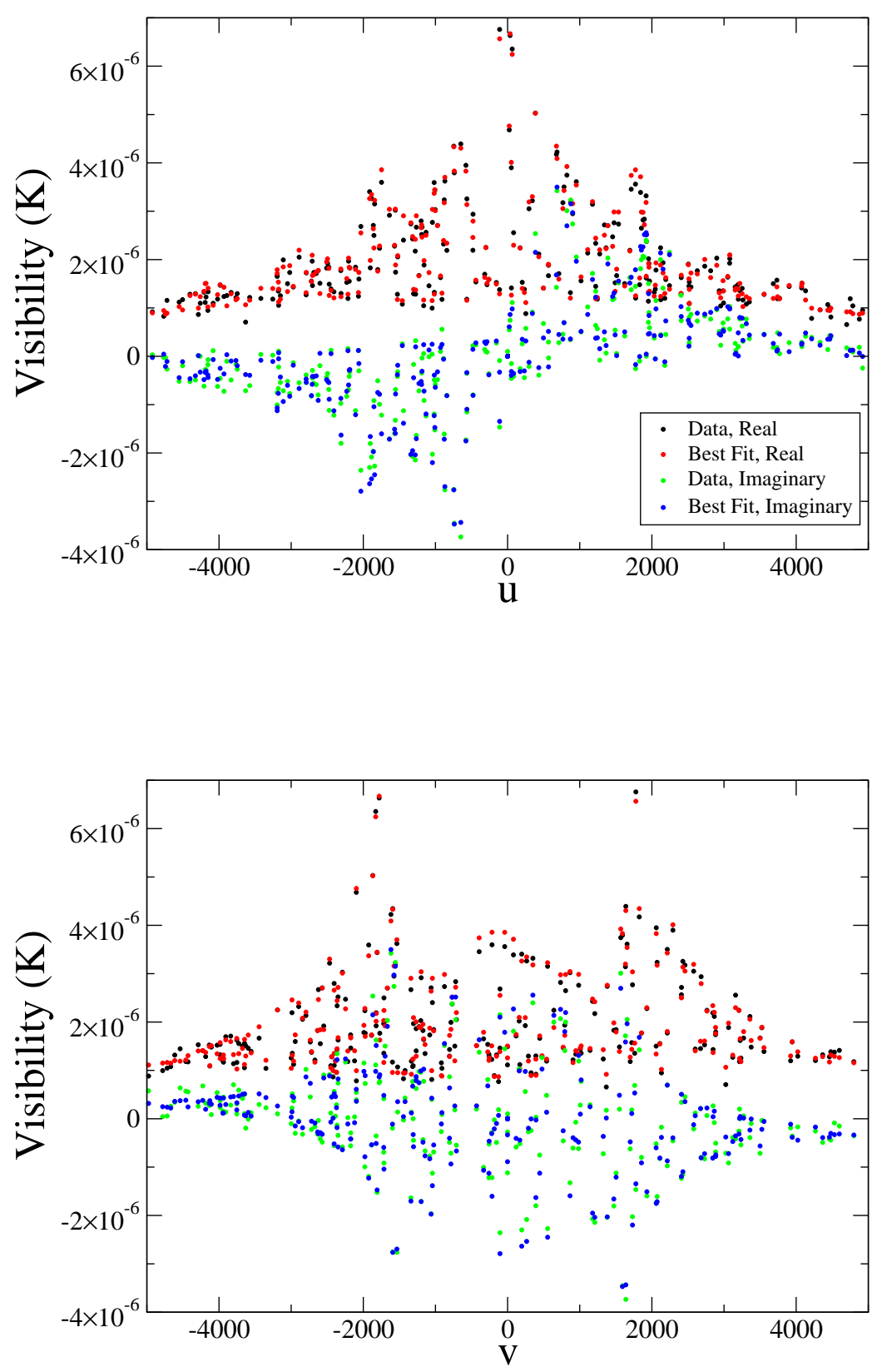

Fig. A.6.- (Top panel) Simulated data projected along $u$ for one of the 10,000 Monte Carlo simulations. Errors are included in the simulated real and imaginary visibility data. The best fitted values are also shown. (Bottom panel) Same as the top panel, but for visibility data projected along $v$. 


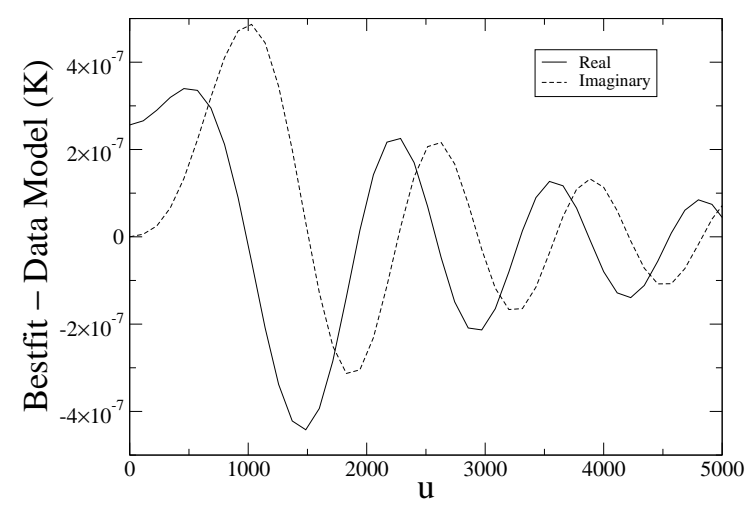

Fig. A.7.- Residuals for positive $u$ along $v=0$ for one of the 10,000 Monte Carlo simulations. The residual is defined as the best fitted model minus the raw input data (Figure A.3). Solid line is the real part. Dashed line is the imaginary part. 


\section{References}

Abell, G. O. 1958, ApJS, 3, 211

Abell, G. O., Corwin, Jr., H. G., \& Olowin, R. P. 1989, ApJS, 70, 1

Afshordi, N., Lin, Y.-T., Nagai, D., \& Sanderson, A. J. R. 2007, MNRAS, 378, 293

Allen, S. W., Schmidt, R. W., \& Fabian, A. C. 2002, MNRAS, 334, L11

Allen, S. W., Rapetti, D. A., Schmidt, R. W., Ebeling, H., Morris, R. G., \& Fabian, A. C. 2008, MNRAS, 383,879

Akahori, T., \& Yoshikawa, K. 2008, PASJ, 60, L19

Akahori, T., \& Yoshikawa, K. 2010, PASJ, 62, 335

Anders, E. \& Grevesse, N. 1989, Geochim. Cosmochim. Acta, 53, 197

Arnaud, M., Neumann, D. M., Aghanim, N., Gastaud, R., Majerowicz, S., \& Hughes, J. P. 2001, A\&A, 365, L80

Arnaud, M., et al. 2002, A\&A, 390, 27

Arnaud, M., Pratt, G. W., Piffaretti, R., Böhringer, H., Croston, J. H., \& Pointecouteau, E. 2009, arXiv:0910.1234 
Bartlett, J. G., Chamballu, A., Melin, J.-B., Arnaud, M., \& Members of the Planck Working Group 5 2008, Astron. Nachr., 329, 147

Basu, K., et al. 2010, A\&A, in press (arXiv:0911.3905)

Batuski, D. J., \& Burns, J. O. 1985a, ApJ, 299, 5

-. 1985b, AJ, 90, 1413

Bautz, M. W., et al. 2010, PASJ, 61, 1117

Bertschinger, E. 1985, ApJS, 58, 39

Birkinshaw, M. 1999, Phys. Rep., 310, 97

Blanton, E. L., Sarazin, C. L., McNamara, B. R., \& Wise, M. W. 2001, ApJ, 558, L15

Blasi, P., Gabici, S., \& Brunetti, G. 2007, International Journal of Modern Physics A, 22,681

Böhringer, H., et al. 2001, A\&A, 365, L181

Böhringer, H., \& Werner, N. 2010, A\&A Rev., 18, 127

Bonamente, M., Joy, M., LaRoque, S. J., Carlstrom, J. E., Nagai, D., \& Marrone, D. P. 2008, ApJ, 675, 106

Bond, J. R., Cole, S., Efstathiou, G., \& Kaiser, N. 1991, ApJ, 379, 440

Borgani, S., \& Kravtsov, A. 2009, Advanced Science Letters, in press (arXiv:0906.4370)

Borkowski, K. J., Lyerly, W. J., \& Reynolds, S. P. 2001, ApJ, 548, 820 
Borkowski, K. J., Sarazin, C. L., \& Blondin, J. M. 1994, ApJ, 429, 710

Bridle, A. H., \& Schwab, F. R. 1999, in ASP Conf. Ser. 180, Synthesis Imaging in Radio Astronomy II, ed. G. B. Taylor, C. L. Carilli, \& R. A. Perley (San Francisco: ASP), 180, 371

Brunetti, G. 2004, Journal of Korean Astronomical Society, 37, 493

Bryan, G. L., \& Norman, M. L. 1998, ApJ, 495, 80

Bullock, J. S., Kolatt, T. S., Sigad, Y., Somerville, R. S., Kravtsov, A. V., Klypin, A. A., Primack, J. R., \& Dekel, A. 2001, MNRAS, 321, 559

Buote, D. A., Gastaldello, F., Humphrey, P. J., Zappacosta, L., Bullock, J. S., Brighenti, F., \& Mathews, W. G. 2007, ApJ, 664, 123

Burns, J. O. 1990, AJ, 99, 14

Bykov, A. M., Dolag, K., \& Durret, F. 2008a, Space Science Reviews, 134, 119

Bykov, A. M., Paerels, F. B. S., \& Petrosian, V. 2008b, Space Science Reviews, 134, 141

Carlstrom, J. E., Holder, G. P., \& Reese, E. D. 2002, ARA\&A, 40, 643

Cen, R., \& Fang, T. 2006, ApJ, 650, 573

Chandran, B. D. G., \& Maron, J. L. 2004, ApJ, 602, 170

Chen, Y., Reiprich, T. H., Böhringer, H., Ikebe, Y., \& Zhang, Y.-Y. 2007, A\&A, 466, 805

Chieze, J.-P., Alimi, J.-M., \& Teyssier, R. 1998, ApJ, 495, 630 
Churazov, E., Brüggen, M., Kaiser, C. R., Böhringer, H., \& Forman, W. 2001, ApJ, 554,261

Churazov, E. and Forman, W. and Jones, C. and Böhringer, H. 2000, A\&A, 356, 788

Chuzhoy, L., \& Loeb, A. 2004, MNRAS, 349, L13

Clarke, T. E., Sarazin, C. L., Blanton, E. L., Neumann, D. M., \& Kassim, N. E. 2005, ApJ, 625, 748

Clowe, D., Gonzalez, A., \& Markevitch, M. 2004, ApJ, 604, 596

Dai, X., Bregman, J. N., Kochanek, C. S., \& Rasia, E. 2010, ApJ, 719, 119

David, L. P., Jones, C., \& Forman, W. 1995, ApJ, 445, 578

Dickey, J. M., \& Lockman, F. J. 1990, ARA\&A, 28, 215

Dolag, K., Bartelmann, M., \& Lesch, H. 2002, A\&A, 387, 383

Dolag, K., Bartelmann, M., Perrotta, F., Baccigalupi, C., Moscardini, L., Meneghetti, M., \& Tormen, G. 2004, A\&A, 416, 853

Einasto, M., Einasto, J., Tago, E., Müller, V., \& Andernach, H. 2001, AJ, 122, 2222

Evrard, A. E., et al. 2008, ApJ, 672, 122

Ettori, S. 2003, MNRAS, 344. L13

Ettori, S., \& Fabian, A. C. 1998, MNRAS, 293, L33

Ettori, S., \& Fabian, A. C. 1999, MNRAS, 305, 834

Fabian, A. C. 1994, ARA\&A, 32, 277 
Fabian, A. C., et al. 2000, MNRAS, 318, L65

Fabian, A. C., et al. 2006, MNRAS, 366, 417

Falceta-Gonçalves, D., Caproni, A., Abraham, Z., Teixeira, D. M., \& de Gouveia Dal Pino, E. M. 2010, ApJ, 713, L74

Falco, E. E., et al. 1999, PASP, 111, 438

Fang, T., Croft, R. A. C., Sanders, W. T., Houck, J., Davé, R., Katz, N., Weinberg, D. H., \& Hernquist, L. 2005, ApJ, 623, 612

Fedeli, C., Moscardini, L., \& Matarrese, S. 2009, MNRAS, 397, 1125

Feretti, L., \& Giovannini, G. 1996, in IAU Symp. 175: Extragalactic Radio Sources, ed. R. D. Ekers, C. Fanti, \& L. Padrielli, 333

Feretti, L., \& Giovannini, G. 2008, in A Pan-Chromatic View of Clusters of Galaxies and the Large-Scale Structure, ed. M. Plionis, O. López-Cruz, \& D. Hughes (Berlin: Springer), 143

Fox, D. C., \& Loeb, A. 1997, ApJ, 491, 459

Fujita, Y., Sarazin, C. L., Kempner, J. C., Rudnick, L., Slee, O. B., Roy, A. L., Andernach, H., \& Ehle, M. 2002, ApJ, 575, 764

Fujita, Y., Sarazin, C. L., Reiprich, T. H., Andernach, H., Ehle, M., Murgia, M., Rudnick, L., \& Slee, O. B. 2004, ApJ, 616, 157

Gao, L., Navarro, J. F., Cole, S., Frenk, C. S., White, S. D. M., Springel, V., Jenkins, \& Neto, A. F. 2008, MNRAS, 387, 536 
George, M. R., Fabian, A. C., Sanders, J. S., Young, A. J., \& Russell, H. R. 2009, MNRAS, 395, 657

Ghavamian, P., Laming, J. M., \& Rakowski, C. E. 2007, ApJ, 654, L69

Giodini, S., et al. 2009, ApJ, 703, 982

Giovanelli, R., \& Haynes, M. P. 1993, AJ, 105, 1271

Gitti, M., Brunetti, G., Feretti, L., \& Setti, G. 2004, A\&A, 417, 1

Gitti, M., Feretti, L., \& Schindler, S. 2006, A\&A, 448, 853

Gonzalez, A. H., Zabludoff, A. I., \& Zaritsky, D. 2005, ApJ, 618, 195

Gonzalez, A. H., Zaritsky, D., \& Zabludoff, A. I. 2007, ApJ, 666, 147

Gunn, J. E, \& Gott, III, J. R. 1972, A\&A Rev., 176, 1

Haardt, F., \& Madau, P. 1996, ApJ, 461, 20

Haiman, Z., Mohr, J. J., \& Holder, G. P. 2001, ApJ, 553, 545

Heinz, S., Brüggen, M., Young, A., \& Levesque, E. 2006, MNRAS, 373, L65

Helfer, T. T., Vogel, S. N., Lugten, J. B., \& Teuben, P. J. 2002, PASP, 114, 350

Holder, G. P., \& Carlstrom, J. E. 2001, ApJ, 558, 515

Holder, G. P., McCarthy, I. G., \& Babul, A. 2007, MNRAS, 382, 1697

Hoshino, A., et al. 2010, PASJ, 62, 371

Hu, W. 2003, Phys. Rev. D, 67, 081304

Hu, W., \& Kravtsov, A. V. 2003, ApJ, 584, 702 
Huchra, J. P., Vogeley, M. S., \& Geller, M. J. 1999, ApJS, 121, 287

Hudson, D. S., Mittal, R., Reiprich, T. H., Nulsen, P. E. J., Andernach, H., \& Sarazin, C. L. 2010, A\&A, 513, A37

Hughes, J. P., \& Helfand, D. J. 1985, ApJ, 291, 544

Hull, A. J., Scudder, J. D., Larson, D. E., \& Lin, R. 2001, J. Geophys. Res., 106, 15711

Jenkins, A., et al. 2001, MNRAS, 321, 372

Kaastra, J. S., et al. 2008, Space Science Reviews, 134, 1

Kaastra, J. S., \& Mewe, R. 1993, A\&AS, 97, 443

Kaastra, J. S., Mewe, R., \& Nieuwenhuijzen, H. 1996, in UV and X-ray Spectroscopy of Astrophysical and Laboratory Plasmas, ed. K. Yamashita \& T. Watanabe (Tokyo: Universal Academy Press), 411

Kang, H., Ryu, D., Cen, R., \& Ostriker, J. P. 2007, ApJ, 669, 729

Karachentsev, I. D., \& Kopylov, A. I. 1981, Soviet Astronomy Letters, 7, 285

Kocsis, B., Haiman, Z., \& Frei, Z. 2005, ApJ, 623, 632

Komatsu, E., et al. 2010, arXiv:1001.4538

Kriss, G. A., Cioffi, D. F., \& Canizares, C. R. 1983, ApJ, 272, 439

Kuntz, K. D., \& Snowden, S. L. 2000, ApJ, 543, 195

Lacey, C., \& Cole, S. 1993, MNRAS, 262, 627 
LaRoque, S. J., Bonamente, M., Carlstrom, J. E., Joy, M. K., Nagai, D., Reese, E. D., \& Dawson, K. S. 2006, ApJ, 652, 917

Lazarian, A. 2006, ApJ, 645, L25

Levine, E. S., Schulz, A. E., \& White, M. 2002, ApJ, 577, 569

Li, Y., Mo, H. J., van den Bosch, F. C., \& Lin, W. P. 2007, MNRAS, 379, 689

Liedahl, D. A., Osterheld, A. L., \& Goldstein, W. H. 1995, ApJ, 438, L115

Loeb, A. 2002, New Astronomy, 7, 279

Loeb, A. 2007, Journal of Cosmology and Astro-Particle Physics, 3, 1

Lueker, M., et al. 2009, arXiv:0912.4317

Lukić, Z., Heitmann, K., Habib, S., Bashinsky, S., \& Ricker, P. M. 2007, ApJ, 671, 1160

Lumb, D. H., Warwick, R. S., Page, M., \& De Luca, A. 2002, A\&A, 389, 93

Majumdar, S., \& Mohr, J. J. 2003, ApJ, 585, 603

Makino, N., Sasaki, S., \& Suto, Y. 1998, ApJ, 497, 555

Mantz, A., Allen, S. W., Ebeling, H., \& Rapetti, D. 2008, MNRAS, 387, 1179

Markevitch, M. 2002, arXiv:astro-ph/0205333

Markevitch, M., et al. 2003, ApJ, 583, 70

Markevitch, M., Forman, W. R., Sarazin, C. L., \& Vikhlinin, A. 1998, ApJ, 503, 77

Markevitch, M., et al. 2004, ApJ, 606, 819 
Markevitch, M., \& Vikhlinin, A. 2007, Phys. Rep., 443, 1

Marković, T., Owen, F. N., \& Eilek, J. A. 2004, in The Riddle of Cooling Flows in Galaxies and Clusters of Galaxies, ed. T. Reiprich, J. Kempner, \& N. Soker (Charlottesville: University of Virginia), 61

Mason, B. S., et al. 2010, ApJ, 716, 739

Mazzotta, P., Mazzitelli, G., Colafrancesco, S., \& Vittorio, N. 1998, A\&AS, 133, 403

Mazzotta, P., Rasia, E., Moscardini, L., \& Tormen, G. 2004, MNRAS, 354, 10

McCammon, D., et al. 2002, ApJ, 576, 188

McNamara, B. R., \& Nulsen, P. E. J. 2007, ARA\&A, 45, 117

McNamara, B. R., et al. 2001, ApJ, 562, L149

Medvedev, M. V. 2007, ApJ, 662, L11

Melin, J.-B., Bartlett, J. G., \& Delabrouille, J. 2005, A\&A, 429, 417

Mewe, R., Gronenschild, E. H. B. M., \& van den Oord, G. H. J. 1985, A\&AS, 62, 197

Mignone, A., Bodo, G., Massaglia, S., Matsakos, T., Tesileanu, O., Zanni, C., \& Ferrari, A. 2007, ApJS, 170, 228

Mohr, J. J., Geller, M. J., \& Wegner, G. 1996, AJ, 112, 1816

Mohr, J. J., \& Wegner, G. 1997, AJ, 114, 25

Molnar, S. M., Hearn, N., Haiman, Z., Bryan, G., Evrard, A. E., \& Lake, G. 2009, ApJ, 696, 1640

Murante, G., et al. 2004, ApJ, 607, L83 
Narayan, R., \& Medvedev, M. V. 2001, ApJ, 562, L129

Navarro, J. F., Frenk, C. S., \& White, S. D. M. 1995, MNRAS, 275, 720

Neto, A. F., et al. 2007, MNRAS, 381, 1450

Ocvirk, P., Pichon, C., \& Teyssier, R. 2008, MNRAS, 390, 1326

Pearson, T. J. 1999, in ASP Conf. Ser. 180, Synthesis Imaging in Radio Astronomy II, ed. G. B. Taylor, C. L. Carilli, \& R. A. Perley (San Francisco: ASP), 180, 335

Peterson, J. R., \& Fabian, A. C. 2006, Phys. Rep., 427, 1

Peterson, J. R., Kahn, S. M., Paerels, F. B. S., Kaastra, J. S., Tamura, T., Bleeker, J. A. M., Ferrigno, C., \& Jernigan, J. G. 2003, ApJ, 590, 207

Peterson, J. R., et al. 2001, A\&A, 365, L104

Pfrommer, C., \& Enßlin, T. A. 2004, A\&A, 413, 17

Plagge, T. et al. 2010, ApJ, 716, 1118

Ponman, T. J., Sanderson, A. J. R., \& Finoguenov, A. 2003, MNRAS, 343, 331

Poole, G. B., Babul, A., McCarthy, I. G., Fardal, M. A., Bildfell, C. J., Quinn, T, \& Mahdavi, A. 2007, MNRAS, 380, 437

Press, W. H., \& Schechter, P. 1974, ApJ, 187, 425

Rakowski, C. E., Ghavamian, P., \& Hughes, J. P. 2003, ApJ, 590, 846

Randall, S. W., Sarazin, C. L., \& Ricker, P. M. 2002, ApJ, 577, 579 (RSR)

Randall, S. W., Markevitch, M., Clowe, D., Gonzalez, A. H., \& Bradač, M. 2008, ApJ, 679, 1173 
Raymond, J. C., \& Smith, B. W. 1977, ApJS, 35, 419

Reid, B. A., \& Spergel, D. N. 2006, ApJ, 651, 643

Reiprich, T. H., et al. 2009, A\&A, 501, 899

Rizza, E., Loken, C., Bliton, M., Roettiger, K., Burns, J. O., \& Owen, F. N. 2000, AJ, 119, 21

Robinson, K., et al. 2004, ApJ, 601, 621

Roncarelli, M., Ettori, S., Dolag, K., Moscardini, L., Borgani, S., \& Murante, G. 2006, MNRAS, 373, 1339

Rosati, P., Borgani, S., \& Norman, C. 2002, ARA\&A, 40, 539

Rudd, D. H., \& Nagai, D. 2009, ApJ, 701, L16

Ryu, D., \& Kang, H. 1997, MNRAS, 284, 416

Ryu, D., Kang, H., Hallman, E., \& Jones, T. W. 2003, ApJ, 593, 599

Sarazin, C. L. 1986, Reviews of Modern Physics, 58, 1

Schekochihin, A. A., Cowley, S. C., Kulsrud, R. M., Hammett, G. W., \& Sharma, P. 2005, ApJ, 629, 139

Schekochihin, A. A., Cowley, S. C., Kulsrud, R. M., Rosin, M. S., \& Heinemann, T. 2008, Phys. Rev. Lett., 100, 081301

Schmidt, R. W., \& Allen, S. W. 2007, MNRAS, 379, 209

Schmidt, F., Vikhlinin, A., \& Hu, W. 2009, Phys. Rev. D, 80, 083505

Sheth, R. K., \& Tormen, G. 1999, MNRAS, 308, 119 
Smith, R. K., Bautz, M. W., Bookbinder, J., Garcia, M. R., Guainazzi M., \& Kilbourne, C. A. 2010, SPIE, in press (http://ixo.gsfc.nasa.gov/resources/Published_Papers/DOCS/SPIE_2010/IXObkgnd-SPIE.pdf)

Smith, R. K., Brickhouse, N. S., Liedahl, D. A., \& Raymond, J. C. 2001, ApJ, 556, L91

Smith, R. K., \& Hughes, J. P. 2010, ApJ, 718, 583

Snowden, S. L. 1998, in IAU Colloq. 166: The Local Bubble and Beyond, ed. D. Breitschwerdt, M. J. Freyberg, \& J. Truemper (Berlin: Springer), 103

Snowden, S. L., et al. 1997, ApJ, 485, 125

Spitzer, L. 1962, Physics of Fully Ionized Gases (2nd ed.; New York: Interscience)

Springel, V., et al. 2005, Nature, 435, 629

Sternberg, A., \& Soker, N. 2008, MNRAS, 384, 1327

Sun, M., Voit, G. M., Donahue, M., Jones, C., Forman, W., \& Vikhlinin, A. 2009, ApJ, 693, 1142

Sunyaev, R. A., \& Zeldovich, Y. B. 1972, Comments on Astrophysics and Space Physics, 4, 173

Takizawa, M. 1999, ApJ, 520, 514

Takizawa, M., Sarazin, C. L., Blanton, E. L., \& Taylor, G. B. 2003, ApJ, 595, 142

Tozzi, P., Scharf, C., \& Norman, C. 2000, ApJ, 542, 106 
Umetsu, K., et al. 2009, ApJ, 694, 1643

Vernaleo, J. C., \& Reynolds, C. S. 2006, ApJ, 645, 83

Verner, D. A., Ferland, G. J., Korista, K. T., \& Yakovlev, D. G. 1996, ApJ, 465, 487

Vikhlinin, A. 2006, ApJ, 640, 710

Vikhlinin, A., Kravtsov, A., Forman, W., Jones, C., Markevitch, M., Murray, S. S., \& Van Speybroeck, L. 2006, ApJ, 640, 691

Vikhlinin, A., et al. 2009, ApJ, 692, 1033

Voit, G. M. 2005, Reviews of Modern Physics, 77, 207

Voit, G. M., \& Donahue, M. 2005, ApJ, 634, 955

Wechsler, R. H., Bullock, J. S., Primack, J. R., Kravtsov, A. V., \& Dekel, A. 2002, ApJ, 568, 52

White, D. A., Jones, C., \& Forman, W. 1997, MNRAS, 292, 419

Wik, D. R., Sarazin, C. L., Ricker, P. M., \& Randall, S. W. 2008, ApJ, 680, 17 (WSR)

Wong, K.-W., Sarazin, C. L., Blanton, E. L., \& Reiprich, T. H. 2008, ApJ, 682, 155

Wong, K.-W., Sarazin, C. L., Loeb, A., \& Wik, D. R. 2008, in The Warm \& Hot Universe, ed F. Paerels, http://warmhot.gsfc.nasa.gov/Posters/Poster07_Wong.pdf

Wong, K.-W., \& Sarazin, C. L. 2009, ApJ, 707, 1141

Wong, K.-W., Sarazin, C. L., \& Wik, D. R. 2010, ApJ, 719, 1 
Wrobel, J. M., \& Walker, R. C. 1999, in ASP Conf. Ser. 180, Synthesis Imaging in Radio Astronomy II, ed. G. B. Taylor, C. L. Carilli, \& R. A. Perley (San Francisco: ASP), 180, 171

Xue, Y.-J., \& Wu, X.-P. 2000, MNRAS, 318, 715

Yoshida, N., Furlanetto, S. R., \& Hernquist, L. 2005, ApJ, 618, L91

Yoshikawa, K., \& Sasaki, S. 2006, PASJ, 58, 641

Young, A. J., Wilson, A. S., \& Mundell, C. G. 2002, ApJ, 579, 560

Zhang, Y.-Y., et al. 2006, A\&A, 456, 55

Zhao, D. H., Jing, Y. P., Mo, H. J., \& Börner, G. 2003a, ApJ, 597, L9

Zhao, D. H., Jing, Y. P., Mo, H. J., \& Börner, G. 2009, ApJ, 707, 354

Zhao, D. H., Mo, H. J., Jing, Y. P., \& Börner, G. 2003b, MNRAS, 339, 12

Zucca, E., Zamorani, G., Scaramella, R., \& Vettolani, G. 1993, ApJ, 407, 470

Zwicky, F. 1937, ApJ, 86, 217 